\title{
Idiopathic Pulmonary Fibrosis (IPF); Physical, Psychological, social impact on quality of life, and the impact of pulmonary rehabilitation as a mode of palliative care on Physical and Physiological markers
}

\section{Senanayake, Sameera P.}

How to cite:

Senanayake, Sameera P. (2019) Idiopathic Pulmonary Fibrosis (IPF); Physical, Psychological, social impact on quality of life, and the impact of pulmonary rehabilitation as a mode of palliative care on Physical and Physiological markers. Doctoral thesis, Swansea University.

http://cronfa.swan.ac.uk/Record/cronfa52430

Use policy:

This item is brought to you by Swansea University. Any person downloading material is agreeing to abide by the terms of the repository licence: copies of full text items may be used or reproduced in any format or medium, without prior permission for personal research or study, educational or non-commercial purposes only. The copyright for any work remains with the original author unless otherwise specified. The full-text must not be sold in any format or medium without the formal permission of the copyright holder. Permission for multiple reproductions should be obtained from the original author.

Authors are personally responsible for adhering to copyright and publisher restrictions when uploading content to the repository.

Please link to the metadata record in the Swansea University repository, Cronfa (link given in the citation reference above.) 


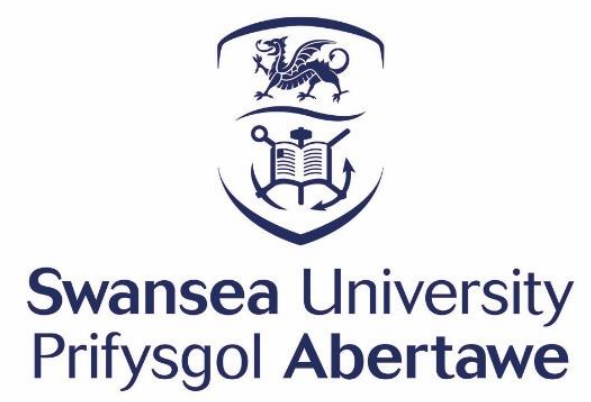

Idiopathic Pulmonary Fibrosis (IPF); Physical, Psychological, social impact on quality of life, and the impact of pulmonary rehabilitation as a mode of palliative care on Physical and Physiological markers.

\section{Sameera Peumal Senanayake}

Submitted to Swansea University in fulfilment for the Degree of Doctor of Philosophy in Sport and Exercise Sciences, Swansea University.

2019. 


\begin{abstract}
Background

Idiopathic Pulmonary Fibrosis (IPF) is a chronic, progressive and life-limiting condition of unknown cause with no curative treatment. The impact of IPF on a patient's quality of life is devastating, yet relatively little attention has been devoted to understanding the disease from a combined physiological and psychological perspective. There are large gaps in our knowledge of the impact of IPF on the quality of life of patients and, importantly, on the effectiveness of palliative care such as pulmonary rehabilitation (PR) programmes.
\end{abstract}

\title{
Methods
}

We initiated this set of investigations by speaking to ten IPF patients recruited from an IPF support group in Swansea, Wales. The aim of this stage of the study was to understand how patients coped with their initial diagnosis, how they live with the disease day-to-day, their experiences and opinions about the support they receive, and their views on any rehabilitation programmes that they were offered. Semi-structured interviews were conducted and an inductive thematic approach was used to analyse the data, allowing us to identify common themes in the patients' experiences. In addition, we sought to identify recommendations for practice that could be applied more globally, to add much needed information that might help to enhance the quality of life for IPF patients in the future.

Secondly, we quantified the effects of an eight-week PR programme for IPF patients, conducted at Morriston Hospital, Swansea. Fifteen individuals (13 with IPF and two with pulmonary fibrosis associated with rheumatoid disease) underwent physical and physiological assessments during a three-day testing protocol: a) On Day 1, physical function (six-minute walk test) and ventilatory function (spirometry) were measured; b) On Day 2, patients' cardio-respiratory responses to slowly-increasing, wide-ranging metabolic challenge (using a protocol consisting of periods of rest, incremental bicycle exercise to maximal effort, and post-exercise recovery) were assessed via respiratory gas analysis and ECG recording; c) On Day 3, patients' cardio-respiratory responses to rapid-onset, high-workload metabolic challenge were assessed (using a protocol including a rapid-onset, constant workload bicycle exercise) by modelling dynamic respiratory oxygen and heart rate responses. Cardiac (RR) interval data were derived from the ECG and used to quantify heart rate variability (HRV) and the cardiac acceleration (AC) and deceleration (DC) capacities (via phase rectified signal averaging, PRSA). Respiratory gas analysis was used to measure the rate of oxygen uptake $\left(\mathrm{V}_{2}\right)$ and oxygen uptake efficiency slopes (OUES). All assessments were performed before and after participants completed the PR programme.

\section{Results}

Inductive analysis of interviews identified seven second-order themes and eleven first-order themes, represented by two General Dimensions: 'Patient experience with the condition' and 'Patient-led recommendations for practice'. The key message on 'coping' in these patients was that acceptance of their condition led to a sense of optimism. Participants reported using appraisal-focused coping strategies to change their perspectives (thinking positively) and emotion-focused strategies to overcome depression (the main opportunity for emotional expression being an IPF support group). The support group also facilitated problemfocused coping: individuals exchanged knowledge and experience and gave one another tips on how live with their condition.

Following the rehabilitation programme, heart rate was elevated by $11-18 \%$ during exercise and recovery states. HRV was not statistically influenced by rehabilitation during any stage of the assessment protocol; however, qualitative changes were apparent with HRV increasing by $68-75 \%$ during all stages of the protocol. Statistically, $A C$ and DC were similar pre- and post-rehabilitation ( $A C=-2.7$ and $-3.2 \mathrm{~ms}$; $D C=2.3$ and $3.2 \mathrm{~ms}$, respectively) but again we observed qualitative improvements in these indices of $19 \%$ and $38 \%$, respectively. 
Post-rehabilitation $\dot{\mathrm{V} O 2}$ was significantly increased ( $p=0.01-0.03$ ) during the first two minutes of heavy-intensity exercise, whilst HR was reduced $(p=0.04)$ during this period. OUES and standard measures of respiratory performance (minute ventilation, peak $\dot{\mathrm{V}} 2$ ) were unchanged following rehabilitation, whilst peak HR and work rates were significantly reduced during incremental exercise only.

\section{Conclusion}

Health professionals should provide patients with information that focuses on living with IPF, encouraging them to make lifestyle changes and adaptations to improve quality of life. Family members should receive education about IPF so that they can support such changes. Patients should be encouraged to join a support group and to participate in physical activity (again preferably group-based). This study offers novel findings that will help inform much-needed changes in the practice of supporting IPF patients to cope with their diagnosis and disease progression.

These studies also provided initial indications that PR programmes might improve heart rate dynamic responses in IPF patients via modulation of the autonomic control of heart rate, even when traditionally-reported respiratory and physical functions are unchanged or diminished. PR also improved the rate of oxygen uptake during heavy-intensity exercise, despite substantially lower heart rates. This suggests that the rehabilitation programme increased systemic arterial-tissue oxygen exchange and/or influenced cardiovascular function to improve systemic oxygen delivery. We might therefore expect that individuals with IPF would find it easier to perform the activities of daily life, including those requiring substantial metabolic demands, following rehabilitation. 


\section{Declaration}

This work has not previously been accepted in substance for any degree and is not being concurrently submitted in candidature for any degree.

Signed (candidate)

Date

This thesis is the result of my own investigations, except where otherwise stated.

Other sources are acknowledged by footnotes giving explicit references. A bibliography is appended.

Signed (candidate)

Date

I hereby give consent for my thesis, if accepted, to be available for photocopying and for inter-library loan, and for the title and summary to be made available to outside organisations.

Signed (candidate)

Date

I hereby give consent for my thesis, if accepted, to be available for photocopying and for inter-library loans after expiry of a bar on access approved by the Swansea University.

Signed (candidate)

Date 


\section{Contents}

$\begin{array}{lr}\text { Abstract } & 02\end{array}$

$\begin{array}{ll}\text { Declaration and statements } & 04\end{array}$

$\begin{array}{lr}\text { Acknowledgements } & 07\end{array}$

$\begin{array}{lr}\text { List of Tables and figures } & 09\end{array}$

$\begin{array}{ll}\text { Definitions and Abbreviations } & 12\end{array}$

$\begin{array}{lr}\text { Chapter } 1 \text { (Introduction) } & 13\end{array}$

Chapter 2 (Literature review): Idiopathic pulmonary fibrosis and the efficacy of pulmonary 20 rehabilitation:

2.1 Background $\quad 20$

$\begin{array}{ll}2.2 \text { Idiopathic pulmonary fibrosis (IPF) } & 20\end{array}$

2.3 Pulmonary rehabilitation (PR) 25

2.4 Evaluating the physiological impact of PR 28

2.5 Evaluating psychological, sociological and physical impacts of the disease on 34 quality of life

Chapter 3 (Methods) 36

3.1 Study 1: Patients' experiences of coping with Idiopathic Pulmonary Fibrosis and 36 their recommendations for its clinical management

3.2 Study 2: Physiological impact of PR for IPF patients 39

Chapter 4 (Patients' experiences of coping with Idiopathic Pulmonary Fibrosis and their 46 recommendations for its clinical management)

$\begin{array}{ll}4.1 \text { Overview } & 46\end{array}$

$\begin{array}{ll}4.2 \text { Results } & 46\end{array}$

$\begin{array}{ll}4.3 \text { Discussion } & 53\end{array}$

$\begin{array}{ll}4.4 \text { Strengths of the study } & 57\end{array}$

4.5 Limitations of the study $\quad 58$

$\begin{array}{lr}4.6 \text { Concluding comments } & 59\end{array}$

Chapter 5 (Heart rate and respiratory responses to incremental (slowly increasing, wide- 60 ranging) metabolic challenge)

$\begin{array}{lr}5.1 \text { Overview } & 60\end{array}$

$\begin{array}{ll}5.2 \text { Participant characteristics } & 61\end{array}$

5.3 Results of physiological assessment 63

$\begin{array}{ll}5.4 \text { Discussion } & 71\end{array}$

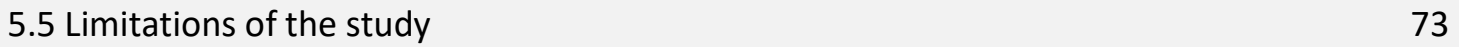

$\begin{array}{ll}5.6 \text { Strengths of the study } & 73\end{array}$

$\begin{array}{ll}5.7 \text { Concluding comments } & 73\end{array}$ 
Chapter 6 (Heart rate kinetics and respiratory responses to extreme (rapid-onset, high

workload) metabolic challenge)

6.1 Overview

6.2 Results

6.3 Discussion

6.4 Strengths and limitations of the study

6.5 Concluding comments

Chapter 7 (Summary and concluding comments)

7.1 Main outcomes

7.2 Clinical relevance and impact

7.3 Future perspectives and recommendations

7.4 Staging and tracking system

7.5 Knowledge transfer

Appendices 


\section{Acknowledgements}

Foremost I would like to express my sincere gratitude to my supervisor Prof. Michael J. Lewis for the continuous support given to me throughout the research project and writing this thesis. I am extremely grateful for his motivation, patience, guidance, immense knowledge and most of all for his overall understanding and maturity on how to supervise a Ph.D student with family commitments. Apart from the theoretical knowledge I learnt the practical aspects on how to become a great academic from you, which I will apply to my own career as a junior academic. I was extremely lucky to have you as my advisor and mentor for my Ph.D study.

Besides my supervisor, I would like to thank my fellow research student, Eri Piastopoulou for the motivation and support given to me with learning to use lab equipment, exercise protocols and the tools to analyse the data I collected over the last three years. Furthermore, I would like to extend my gratitude to Dr. Joanne Hudson, who helped me immensely to complete the qualitative part of my study. It was a pleasure working with you and I learnt a great deal on qualitative research from your teaching in a very short period of time.

I thank Dr. Melitta McNarry (Department of Sport Science, Swansea University), Prof. Kim Harrison, Mr. Simon Hilldrup, Mrs.Caroline Evered (ABMU Health Board) and Mrs. Gwyneth Churchill, Mr. Nathan Welsh and Mr. Kevin Stroke for the support and guidance given to me during my study and stay in Swansea.

I would like to thank Prof, Athula Sumathipala (Keele University U.K.), Col. PTR Makuloluwa (KDU, Sri Lanka) and Dr. David. P. Wilson, (University of Adelaide, Australia) who guided me to secure the Ph.D placement at Swansea University U.K. and both my loving parents (Dr. G.S. Senanayake and Mrs. S.R.M. Senanayake) who gave me the financial assistance and motivation to complete my studies on time.

With all the acknowledgements out of the way I would like to dedicate this thesis to my loving wife, Dr. Amali Senanayake who gave me the motivation, courage and all the support in the world by taking care of our two kids, Anuka and Helika Senanayake all by herself, living half way across the world while I was doing my work in the U.K. Without your support none of this would have been possible and I am extremely grateful and proud of you for the maturity and character shown by you during the past three years.

Finally, I would like to thank my in-laws (Mr. Lalitha Jayasinghe and Mrs. Asoka Jayasinghe) along with Mr. Chanaka Rajapaksha for the support given to us during these tough times. 


\section{Publications relating to the study}

1. Patients' experiences of coping with Idiopathic Pulmonary Fibrosis and their recommendations for its clinical management. Senanayake S, Harrison K, Lewis M, McNarry M, Hudson J. Patients' experiences of coping with Idiopathic Pulmonary Fibrosis and their recommendations for its clinical management. PloS One. 2018;13(5):e0197660.

https://journals.plos.org/plosone/article?id=10.1371/journal.pone.0197660

2. Influence of physical rehabilitation on heart rate dynamics in patients with idiopathic pulmonary fibrosis. Senanayake S, Harrison N, Lewis M. Influence of physical rehabilitation on heart rate dynamics in patients with idiopathic pulmonary fibrosis. Journal of exercise rehabilitation. 2019;15(1):160.

https://www.ncbi.nlm.nih.gov/pmc/articles/PMC6416513/ 


\section{List of Tables and figures}

Table/figure

Heading

Page

number

number

Table 2.1 Diagnostic criteria for IPF without a lung biopsy. 23

Figure $2.1 \quad$ (top) RR interval of a healthy 21-year-old male during rest, exercise and 30 recovery. (bottom): time-frequency plot for the same individual as above.

Figure 2.2 Averaged power distribution during periods of rest, exercise and recovery for the same individual (Fig.2.1).

Table 3.1 Example of the Overview and the purpose of questions asked during patient interviews.

Figure 4.1 Key themes representing patients' experiences of IPF and patient-led

Recommendations for practice.

Table 5.1 Participants' drug regimens during the rehabilitation programme.

Table 5.2

Patient demographics.

Table 5.3

Physiological responses to incremental exercise tests pre- and postrehabilitation.

Table 5.4 HRV indices pre and post PR.

Figure 5.1 HRV indices pre and post PR.

Figure 5.2 Percentage change in HRV indices following PR. 68

Figure 5.3 Group mean (+/-SEM) of the normalized RR time-series around the 69 identified (a) acceleration and (b) deceleration anchor-points.

Table 5.5 Summary results for Acceleration Capacity (AC) and Deceleration 70 Capacity (DC) pre and post PR.

Figure 5.4 Box-and-whisker plot of Acceleration and Deceleration Indices pre and 70 post PR.

Figure 6.1 Example of an individual $\mathrm{V}_{2}$ response during the pre-exercise. 76

Figure 6.2 Example of an individual $\dot{\mathrm{V}}_{2}$ response during the pre-exercise, exercise $\quad 76$ and post-exercise phases of the Day 3 protocol.

Table 6.1 Physiological responses to constant workload tests, pre- and postrehabilitation.

Figure 6.3 $\quad$ (a) Individual $\mathrm{V}_{2}$ responses to constant workload exercise for a selection of participants before PR (b): Normalised individual $\dot{V}_{2}$ responses for the same participants as in (a); (c): individual $\mathrm{VO}_{2}$ responses to the constant workload test, post PR; (d): normalised $\dot{\mathrm{V}}_{2}$ responses for the same participants as in (c).

Figure 6.4 (a) Individual HR responses to constant workload exercise for a selection of participants after PR; (b): Normalised individual HR responses for the same participants as in (a); (c): individual HR responses to the constant workload test, post PR; (d): normalised HR responses for the same participants as in (c).

Table 6.2 Results of group-averaged, normalised $\dot{\mathrm{VO}}_{2}$ and $\mathrm{HR}$ modelling. 
Figure 6.5 The curve fitting process used to model the group mean $\mathrm{V}_{2}$ data

(Matlab curve fitting toolbox): (a) Pre-PR and (b) Post-PR.

Figure 6.6 The curve fitting process used to model the group mean HR data (Matlab 85 curve fitting toolbox): (a) Pre-PR and (b) Post-PR.

Figure 6.7 (a): Group-averaged normalised temporal $\dot{\mathrm{V}}_{2}$ response to constant work rate exercise.

Figure $6.8 \quad$ (a): Group-averaged normalised temporal HR response to constant work rate exercise.

Figure C.1 Example plot of $\dot{\mathrm{VO}}_{2}$ as a function of time for an individual patient (raw data).

Figure C.2 Example plot of $\dot{\mathrm{VO}}_{2}$ against VE, showing the calculation of OUES.

Table C.3 Physiological responses to incremental (maximal effort) exercise tests pre- and post-rehabilitation for each individual who took part in the study.

Table C.4 OUES for pre- and post-PR states.

Table C.5 Acceleration Capacity and Deceleration Capacity indices for each individual participant pre and post PR.

Figure D.1 Modelling of individual participants' temporal $\mathrm{V}_{2}$ data for (a) pre-PR and (b) post-PR conditions.

Figure D.2 Modelling of individual participants' HR data for (a) pre-PR and (b) postPR conditions (a) Pre -PR.

Figure E.1 Proposed staging system for PR for IPF.

Table E.1 Physiological parameters which were selected to be included in the staging system for every individual 


\section{Definitions and Abbreviations}

\section{Abbreviations}

Acceleration capacity (AC)

American Thoracic Society (ATS)

Amyotrophic lateral sclerosis (ALS)

Autonomic nervous system (ANS)

Blood gas analysis (ABG)

British Thoracic Society (BTS)

Carbon monoxide transfer factor $\left(D_{L C O}\right)$

Chronic Obstructive Pulmonary Disease (COPD)

Deceleration capacity' (DC)

Diffusing capacity of carbon monoxide $\left(\mathrm{K}_{\mathrm{co}}\right)$

European Respiratory Society (ERS)

Forced expiratory volume in 1 second $\left(\mathrm{FEV}_{1}\right)$

Forced vital capacity (FVC)

Functional residual capacity (FRC)

Gas Exchange Threshold (GET)

Health-related quality of life (HRQoL)

Heart rate (HR)

Heart rate variability (HRV)

High frequency components (HF)

Idiopathic Pulmonary Fibrosis (IPF)

Low frequency components (LF)

Maximal oxygen uptake ( $\left.\dot{\mathrm{V}}_{2} \max \right)$

Mean response time (MRT)

Millilitres (ml)
Minute ventilation (VE)

Minutes (min)

Oxygen saturation $\left(\mathrm{SaO}_{2}\right)$

Oxygen uptake efficiency slope (OUES)

Peak rate of oxygen uptake $\left(\mathrm{V}_{2}\right)$

Phase rectified signal averaging (PRSA)

Cardiac interval (RR)

Power spectral density (PSD)

Pulmonary rehabilitation (PR)

Quality of Life (QoL) Total lung capacity (TLC)

Respiratory exchange ratio (RER)

Interstitial Lung Disease (ILD)

Root mean square of the standard deviation (RMSSD)

Root mean squared error (RMSSE)

Six-minute walk distance (6MWD)

Standard deviation of the NN intervals (SDNN)

Summed square of residual errors (SSE)

Total power (TP)

Usual interstitial pneumonia (UIP)

Ventilatory equivalent for carbon dioxide $\left(\mathrm{VE} / \mathrm{V} \mathrm{CO}_{2}\right)$

Ventilatory equivalent for oxygen $\left(\mathrm{VE} / \mathrm{VO}_{2}\right)$

Very low frequency components (VLF) 


\section{Physiological Definitions}

Carbon monoxide transfer factor $(\mathrm{ml} / \mathrm{min} / \mathrm{mmHg})$ : Measures the diffusing capacity of the lung for carbon monoxide.

Cardiac interval (ms): The measured variation in the beat-to-beat interval of the heart

Diffusing capacity of carbon monoxide $(\mathrm{mmol} / \mathrm{min} / \mathrm{kPa} / \mathrm{L})$ : Measures the ability of the lungs to transfer gas to red blood cells.

Forced expiratory volume in $\mathbf{1}$ second (L): Amount of air an individual exhales in one second.

Forced vital capacity (L): Total volume of air exhaled in a spirometry test

Gas Exchange Threshold (\%): The point at which cellular respiration switches from aerobic to anaerobic respiration.

Heart rate variability (ms): The variation in heart rate between consecutive heart beats

Heart rate (BPM): Number of times the heart beats in a minute

Maximal oxygen uptake $(\mathrm{ml} / \mathrm{kg} / \mathrm{min})$ : Maximum rate of oxygen consumption during heavy exercise

Minute ventilation (Lpm): Total volume of air a person exchanges in one minute.

Oxygen uptake efficiency slope $\left.\left(\mathrm{ml} \cdot \mathrm{min}^{-1} \cdot \mathrm{L}^{-1}\right)\right)$ : The linear relationship between oxygen uptake and minute ventilation

Phase rectified signal averaging (ms): A technique that can be used to estimate the acceleration and deceleration capacities of the heart

Respiratory exchange ratio: Ratio between the carbon dioxide produced and the oxygen consumed during respiration

Total lung capacity ( $\mathrm{ml})$ : Total amount of air in the lungs after taking the deepest breath possible

Ventilatory equivalent for carbon dioxide: The ratio between minute ventilation and carbon dioxide production

Ventilatory equivalent for oxygen: The ratio between minute ventilation and oxygen production 


\section{Chapter 1}

\section{Introduction}

\section{Chronic lung disease}

There are two primary categories of chronic lung pathology, referred to as 'obstructive' and 'restrictive' lung diseases which impair the function of the human respiratory system. 'Obstructive diseases such as Chronic Obstructive Pulmonary Disease (COPD) and restrictive diseases such as Interstitial Lung Disease (ILD) both reduce an individual's ability to 'breathe' (that is, to uptake and use oxygen) but their mechanisms of action differ significantly. Obstructive disease causes physical airway (bronchial) restriction (lumen narrowing) whilst restrictive disease impedes alveolar stretching $[1,2]$.

Idiopathic Pulmonary Fibrosis (IPF) is the commonest of the interstitial lung diseases (ILD) [3]; it causes progressive fibrosis of the alveolar walls and severe impairment of lung function [3]. The median survival for patients diagnosed with IPF is 2.5 to 3.5 years but the range is wide, with some individuals following a slowly declining trajectory over many years and others experiencing rapid disease progression [4]. Histologically, it is characterised by deposition of excess extracellular matrix within the alveolar interstitium and fibroblastic foci. These changes, described as usual interstitial pneumonia (UIP), have a typically peripheral, basal distribution which can be observed on CT scanning of the thorax [3]. Interestingly, similar pathological changes are sometimes observed in patients with collagen vascular disease such as rheumatoid arthritis. Patients with UIP usually present with symptoms of increasing breathlessness and fatigue which results in difficulty conducting day-to-day activities and a reduced quality of life [5]. COPD is an umbrella term used to describe progressive lung diseases including emphysema, chronic bronchitis, and refractory (non-reversible) asthma. COPD is a progressive disease characterized by increasing breathlessness and, whilst it remains incurable, there are several treatments that can relieve their symptoms [6]. These include oxygen therapy and bronchodilator medication (both of which give rapid and effective but temporary relief) and longer-term therapies such as pulmonary rehabilitation (PR). PR can be very effective in managing the symptoms of COPD [7], allowing individuals with COPD to have a relatively normal day-to-day life [7]. Unfortunately, unlike in COPD, the physical changes that define restrictive lung diseases (UIP characteristics) are not reversible, although new anti-fibrotic therapies that slow the decline in lung function in these diseases are now available [3]. National guidelines also recommend that $\mathrm{PR}$ is offered to help improve functional performance in patients with restrictive lung disease $[8]$. 
The primary goal of physical exercise-based PR is to "restore patients to the highest possible functional state" [9]. Whilst PR can improve exercise performance and lung function in other forms of chronic lung disease (such as COPD) [8], evidence of its influence on IPF patients is limited [10] but growing [11, 12]. Two reports have noted improved six-minute walk distance (6MWD), health-related quality of life (HRQoL) and reduced symptom severity in individuals with ILD following eight weeks of PR [13]. However some authors have suggested that PR might not be effective for IPF patients when compared with COPD [10]14, 15]. This is because any improvements obtained from PR cannot be sustained over time in IPF patients (typically being reversed within months after completion of the programme), and because of the progressive nature of the disease $[10,14,15]$. Nevertheless, the physiological mechanisms by which the initial improvements are achieved are important, but little understood. Hence is it important to look into this to better understand both the IPF patient's response to exercise at a physiological level and the degree of improvement of these variables following a sustained period of rehabilitation.

A fundamental confounder in evaluating the effectiveness of PR as a therapy for restrictive lung disease is that, despite the different pathophysiological characteristics of obstructive and restrictive lung pathology, patients with either of these diseases are typically (almost always) offered identical PR programmes. PR typically includes an educational component and a physical exercise component, which is typically one hour long, during which 10 minutes are spend warming up and cooling down and the rest spent on bouts of toning exercises (weights) and cardiovascular exercises (treadmill / bicycle ergometer). One might argue that this approach is not optimal, or even that it is not appropriate for IPF patients: why would we expect that physical exercise could reverse the influences of fibrotic changes in the lung? However, the value of PR should not be interpreted solely in terms of measurable physiological improvements or anatomical changes; arguably the most relevant potential benefit of PR in patients with restrictive disease is an improvement in their day-to-day quality of life. We might speculate that PR would contribute to this via factors such as improved social interaction (such as attending group PR sessions), increased confidence in undertaking physical activity (reinforced by performance in a supervised PR session) and improved perceptions of stamina related to the above. 


\section{Outcome measures to assess the efficacy of $P R$}

Mindfulness of a patient's Quality of Life (QoL) is an important factor that should underpin the holistic care of people with IPF, of which PR is just one part. From a healthcare perspective it is important to try to understand the psychological impact of an IPF diagnosis: patients are told that they have a life-limiting disease (that is littlerecognised and poorly-understood by the general public) and that there are no curative options [1]. From a patient's perspective a diagnosis of IPF is therefore often completely unexpected and confusing, provoking a range of psychological responses and causing much anxiety. Healthcare professionals have an important role in helping to improve or preserve QoL, taking into account the impact of physical, psychological and social wellbeing [4, 5]. A recent review [6] highlighted studies that have attempted to measure or improve QoL in IPF (using patient-reported outcome measures and interventions) and those that have identified unmet patient needs (such as emotional support and information resources). A few studies have assessed the individual psychological impact of an IPF diagnosis and patients' experiences of living with the disease [7-11].

The single best measure of an individual's aerobic fitness (capacity for oxygen utilisation) is considered to be their peak or maximal oxygen uptake $\left(\dot{\mathrm{V}}_{2}\right.$ max). However, in practice the measurement of $\dot{\mathrm{V}}_{2}$ max can be difficult - for example in older people with impaired mobility or in people with cardiorespiratory disease and limited functional capacity. Previous research by colleagues in Swansea University has reported that values for the peak rate of oxygen uptake in people with IPF are half those of similarly aged healthy individuals, whilst their peak heart rates (HR) are $20 \%$ lower [16]. These results imply that there is reduced tissue oxygen utilization following the onset of exercise, which in large part results from impeded central (cardiovascular) oxygen delivery. However, it is not known whether PR can improve systemic oxygen delivery and utilisation in people with IPF and other causes of UIP.

Alternatives to measuring $\dot{\mathrm{V}}_{2}$ max have been developed to help overcome the difficulties often encountered in its measurement in patients. One of these, the 'oxygen uptake efficiency slope' (OUES), quantifies the body's oxygen uptake and extraction efficiency as the rate of increase of oxygen uptake $\left(\mathrm{V}_{2}\right)$ in response to increases in minute ventilation (VE) during incremental exercise. OUES has several advantages over other measures of aerobic exercise performance: it can be determined accurately from submaximal exercise protocols (below 60$75 \%$ functional reserve) yet is strongly correlated with $\dot{\mathrm{V}}_{2}$ max; it is completely objective (tester-independent) and it has better inter-protocol agreement [17]19]. However, whilst OUES has been quantified in various populations including children, adults with heart disease and elite athletes [19]22] it has not been used to study patients with chronic lung disease. An alternative to OUES is the analysis of so-called 'pulmonary oxygen 
uptake kinetics', which involves mathematical modelling of the rate of change of oxygen utilisation in response to a sudden change in exercise workload. This provides insight into an individual's ability to absorb and utilize oxygen to fuel a change in metabolic activity [23]. Several studies have explored the influence of PR on oxygen uptake kinetics in COPD [10]. Puente-Maestu et al. [24] examined the effects of supervised and unsupervised eight-week PR programmes on a mono-exponential oxygen uptake model and observed an increase in the mean endurance time at a work rate equivalent to $70 \%$ of $\mathrm{VO}_{2}$ max in both groups, whilst $\mathrm{CO}_{2}$ output, minute ventilation and heart rate were also observed to have increased post training. Few studies have applied this technique to patients with ILD. McNarry et al. [16] compared the oxygen uptake response in patients with emphysema and IPF and found that both displayed $\mathrm{VO}_{2}$ kinetics that were similarly slow in comparison to controls. A study of 11 patients with IPF also observed improvement in treadmill exercise time and maintained oxygen consumption following a three-month PR programme, whereas controls suffered a decline in $\mathrm{V}_{2}$ kinetics [25]. Other reports have noted improvements in exercise tolerance, dyspnoea and quality of life in people with IPF who undergo PR $[3,14,25]$, but the physiological mechanisms by which such improvements occur are not understood.

Heart rate dynamics are thought to provide a good proxy for the response of cardiac output and muscle blood flow to increased metabolic demand [26, 27], albeit they do not account for the contribution of stroke volume in meeting this demand [27]. One way to determine HR responsiveness is to measure the variation in beat-tobeat duration of the cardiac cycle or heart rate variability (HRV) [28]. This technique has been widely used to estimate the influences of the autonomic nervous system (ANS) on heart rate in various populations. However, caution must be applied when HRV is used to determine an individual's capacity to increase or decrease heart rate. Firstly, because HRV must be determined during steady-state conditions and extended assessment periods. Hence, it cannot provide an overall measure of steady-state conditions and extended assessment periods or of the range of cardiac dynamics that occur across multiple states or that are transient in nature. Secondly, the use of HRV as an independent measure of sympathetic and parasympathetic control is not straightforward [28]. An alternative measure of cardiac dynamics that avoids these restrictions is the use of phase rectified signal averaging (PRSA) [29]. This method yields averaged RR time series that separately characterize all heart rate modulations that result in accelerations and decelerations. It is known that individuals with COPD have a degree of cardiac autonomic dysfunction, reflected by altered HRV during rest and exercise [30] but with a normal diurnal variation [31]. They also have a tendency towards reduced PRSA-derived cardiac dynamic capacities that are ameliorated with oxygen therapy [31]. However, neither HRV nor cardiac acceleration/deceleration capacities have been evaluated in individuals with UIP. A comparison of these 
variables before and after physical rehabilitation might indicate whether these patients would benefit from PR interventions in terms of improved cardiac responsiveness to everyday activities.

\section{Aims of the thesis}

Although several qualitative studies have been conducted on patients' experiences with IPF most of these have focused on the impact of specific aspects (breathlessness, fatigue, dyspnoea) of the disease on quality of life, but not the overall psychological and the sociological impact of IPF on the individual patient. Hence chapter 4 of this thesis describes a qualitative investigation that sought to extend our knowledge by synthesising an 'overall impact' of the disease - that is, integrating its individual impacts on physical, psychological and social wellbeing in daily life. The aim of this study was to explore the idiographic ${ }^{1}$ experiences of patients with IPF within our local health board in South West Wales, and thus to improve our understanding of the psychosocial impact of the disease in this region. In so doing, we hoped to gain insight into how to best support the psychological needs of individuals diagnosed with IPF. The study offers novel findings that will help to inform much-needed local changes in the practice of supporting IPF patients to cope with their diagnosis and disease progression.

The focus of Chapter 5 is the comparison of HRV and heart rate responsiveness in ILD patients before and after an exercise-based PR programme. HRV analysis was chosen as a tool to estimate cardiac autonomic nervous system function during steady-state conditions, whilst cardiac acceleration and deceleration capacities (derived using the PRSA method) provided estimates of overall heart rate responsiveness across multiple physiological conditions. This is the first study to report these descriptors of heart rate dynamics in individuals with restrictive lung disease. In this context, the aim of this study was to determine the influence of an eightweek, exercise-based PR programme on measures of cardiac autonomic control in patients with UIP. Specifically, we sought to quantify HRV and indices of cardiac acceleration and deceleration capacity for these individuals during periods of rest and exercise.

The drivers for the investigations described in Chapter 6 of this thesis were, i) a desire to investigate the influence of PR on patients' capacities to respond to extreme (high-intensity, rapid onset) metabolic challenge, as encountered, for example, during some physical activities of daily living, and ii) a recognition that any meaningful assessment of the physiological efficacy of PR in restrictive lung disease requires the quantification

\footnotetext{
${ }^{1}$ In psychology, the term idiographic describes the study of the individual, who is seen as a unique agent with a unique life history, with properties setting him/her apart from other individuals.
} 
of multiple physiological biomarkers. In particular, it was speculated that any such influence would involve not only respiratory function but also cardiac function, taking account of the inter-dependent nature of the ventilation-perfusion and arterial-tissue oxygen exchange mechanisms. Thus, in an attempt to estimate their capacities to cope with extreme metabolic challenge, the aim of the investigations reported in this chapter was to quantify patients' dynamic cardiorespiratory responses (oxygen uptake kinetics and heart rate kinetics) to a sudden and substantial metabolic challenge, before and after PR.

\section{Summary}

Studies conducted on the outcomes of PR for IPF patients are limited, and most have quantified the outcomes of PR using functional measures such as the six-minute walk distance, the dyspnoea scale and maximum oxygen uptake. Although some studies have indicated improvements in these parameters, they have been of limited value in providing evidence for the efficacy of PR. In particular they did not adequately consider the physiological impact of PR, giving rise to a gap in the knowledge of the physiological adaptability of IPF patients in response to physical rehabilitation. This thesis will address this gap, as well as extending the concept of the 'efficacy' of PR by investigating patients' perceptions of its psychological and sociological impact. 


\section{Chapter 2}

\section{Idiopathic pulmonary fibrosis and the efficacy of pulmonary rehabilitation:}

\section{Literature review}

\subsection{Background}

Interstitial lung disease (ILD) is an umbrella term given to diseases including IPF , amyotrophic lateral sclerosis (ALS) and sarcoidosis, each of which is a restrictive pulmonary pathology [13]. According to the American and European respiratory societies ILD can be categorised under five main groups [32]:

1. Exposure-related ILDs (occupational, environmental, avocational and due to medication)

2. Connective tissue related ILDs (scleroderma, rheumatoid disease and Sjogren's syndrome)

3. ILDs caused by granulomas (sarcoidosis)

4. ILDs caused by various other known disorders (pneumonia, vasculitis)

5. ILDs of unknown cause (idiopathic interstitial pneumonia).

The American Thoracic Society and the European Respiratory Society further classify idiopathic interstitial pneumonia into two groups: 'Major' idiopathic interstitial pneumonia (which includes IPF) and 'Rare' idiopathic interstitial pneumonia [3].

IPF is a chronic, progressive and life-threatening condition of unknown cause and the commonest of the Idiopathic Interstitial Pneumonias. In the UK there are 32,500 people affected by IPF with 6000 fresh cases of IPF reporting every year, with a prevalence of 50 per 100,000 people [33]. The histological pattern of IPF is characterised by the deposition of excess collagen and other molecules of the extracellular matrix within the alveolar interstitium, together with a modest inflammatory cell infiltrate [36]. This pathological process results in a gradual loss of functioning alveolar units and a decline in pulmonary function. Consequently, people with IPF develop progressive breathlessness and eventually die from respiratory failure. Whilst new therapies which slow the decline in lung function have recently become available, there is still no cure for IPF which has a median survival of three years although the range is wide; some patients follow a slowly declining trajectory over many years whilst others may have a rapidly progressive course [37]. The majority of patients will therefore require supportive care at some point in the course of their disease and national guidelines recommend that oxygen therapy, PR and palliative care should be offered when appropriate (NICE 2013) [10]. 
PR programmes are of proven value in the management of patients with COPD in whom they improve exercise performance and quality of life [8]. However, the benefit of such programmes in terms of improving exercise performance in people with IPF is less clear [8]. With this in mind, the aims of this chapter are to provide an overview of IPF, to explore previous research on the effectiveness of PR for IPF patients, and to consider the potential benefits (physiological and psychological) of PR and how best to measure these. In this way the present chapter will help to explain the rationale for the studies reported in this thesis (Chapters 4-6) as well as supporting the methods employed (Chapter 3).

\subsection{Idiopathic pulmonary fibrosis}

\subsubsection{Classification}

IPF is a chronic, progressive, irreversible and life threatening condition of unknown cause and the commonest of the Idiopathic Interstitial Pneumonias [38]. IPF may also be referred to as 'usual interstitial pneumonia' (UIP). It is characterised by progressive fibrosis without substantial inflammation [39] and is common in the middleaged and elderly populations. IPF patients have a median age of 66 years [38].

\subsubsection{Aetiology of IPF}

IPF is believed to result from a range of diseases such as scleroderma, sarcoidosis, and inflammation due to infection, and also from environmental factors such as exposure to silica, metal, wood and asbestos dusts [39]. Other major causes of IPF are, cigarette smoking [40], medication such as antidepressants [40], infectious agents [41] and chronic aspiration [42],[43] and viral infections [44]. These infections include hepatitis C, Epstein-bar virus, and adenovirus [44]. Although familial IPF is rare, genetic factors are also considered a cause of IPF [45].

\subsubsection{Epidemiology of IPF}

According to Raghu et al. [46], the prevalence of IPF is higher in men than women (in the ratio 1.5:1.7) and this increases with age. A study conducted in New Mexico among almost half a million people for a duration of two years found the incidence ratio of the disease to be 11:7 (men: women) and the prevalence ratio to be 20:13 (men: women) [47]. Another study conducted in Japan found that the highest prevalence of IPF (28 per 100,000 population) is in the age group 60-69 years and was lowest (5 per 100,000 population) in the age group 20-49 years [48]. A statistically definitive epidemiological study conducted by the British Lung Foundation through their 'respiratory health of a nation' project [33] looked in to the prevalence and incidence rates of IPF through the Health Intelligence network which represents $5 \%$ of the population. They calculated the mortality figures through official government statistics and their results showed that an estimated 32,500 people in the UK are 
suffering from IPF, with 6000 new cases reported every year where the incidence is increased with age (85\% of diagnoses are among people who are over 70 years old). The prevalence was highest in Northern Ireland, NorthWest England, Scotland and Wales. Furthermore the study also revealed that there are 9000 hospital admission every year with IPF with an annual mortality of 5,300 [33]. The study further finds that IPF is $50 \%$ more common among males and it had killed $60 \%$ more men compared to women between the years of $2008-2012$. The study claims that IPF is common than previously recognised leading to a small but significant burden on the NHS. Another study conducted in the UK by Gribbin et al [34]. analysed a primary care database of roughly four million patients and found that there were 962 cases of IPF reported between the years 1991 and 2003. The data showed a crude incidence rate of 4.6 per 100,000 and a rise of $11 \%$ in the incidence of IPF during that period. However, this notably large increase in incidence likely reflects an improvement in diagnostic methods over this period (with high resolution tomography introduced in the early 1990's) and more patients being diagnosed specifically with IPF, rather than a true increase in the incidence rate of IPF.

\subsubsection{Disease Progression}

Following diagnosis with IPF the life expectancy of a patient is a median of three years [49]. Before diagnosis every patient has an asymptomatic period which will vary from months to years, during which he/she is exposed to micro injuries to the lung tissue until the onset of symptoms [38]. Once diagnosed there are three different heterogeneous clinical courses (clinical phenotypes) that a patient could take during the remainder of their lives (three distinct paths of health decline) [38]: 1) Rapid progressive type, 2) Acute exacerbation type, and 3) Stable or slow progressive type. The worst prognoses are for older individuals ( $>70$ years), those of low body mass index (BMI), those with a history of smoking, and those with severe physiological impairment and pulmonary hypertension [4].

1. Rapid progressive: This subgroup of patients are usually male smokers with a high BMI. Also known as accelerated IPF, this is a relatively uncommon course. Death occurs within $2-3$ years despite the fact that it has similar lung function, chest imaging and histological findings as those with the slow progressive form [38].

2. Acute exacerbation: This subgroup is defined by the rapid deterioration of the patient's condition in the absence of known triggers such as infections, pulmonary embolism, or heart failure [50] [51]. This accounts for $5-20 \%$ of IPF cases $[52,53$. These patients have a mortality of more than $60 \%$ during hospital admission following exacerbation, and for those who survive the mortality is more than $90 \%$ within six months [53]. 
3. Stable or slow progressive: Most of the patients diagnosed with IPF fall under this category of the disease, and they require medical care for months or years after diagnosis [38]. Symptoms in these patients include cough and dyspnoea, and reduced lung volumes and capacities lead to hypoxaemia that increases with exercise [38].

\subsubsection{Pathogenesis}

The histological pattern of IPF is characterised by the deposition of extracellular matrix in the alveolar interstitium. The exact cause of IPF is still unknown, but the "inflammatory/alveolitis" hypothesis was widely accepted for decades [45]. In this hypothesis it was assumed that IPF is a chronic inflammatory disease due to unknown stimulus and when left untreated it would lead to progressive lung disease and ultimately cause fibrosis [54], [55]. However this theory was discarded when evidence showed that IPF responded poorly to longterm anti-inflammatory therapy [56][58].

The current hypothesis, known as the "epithelial/mesenchymal" hypothesis, describes IPF as a disease which arises due to epithelial cell activation from unknown exogenous and endogenous (micro injury) stimuli [59]. These injuries could result from smoking, viruses, wood and metal dust, oxidants and ageing, which in turn cause disruption of the alveolar epithelium [60]. This results in the constant migration, activation and proliferation of mesenchymal cells and the subsequent formation of fibroblastic foci in the alveoli. This leads to the accumulation and deposition of extracellular matrix, causing abnormal wound repair [59] and leading to deposition of excess collagen and other molecules of the extracellular matrix within the alveolar interstitium [36]. This pathological process results in a gradual loss of functioning alveolar units and a decline in pulmonary function.

\subsubsection{Diagnosis}

Diagnosis of the disease is complex and is key to developing an appropriate management plan [45]. The diagnostic criteria shown in Table 2.1 are those presented in the American Thoracic Society guidelines [9]. 


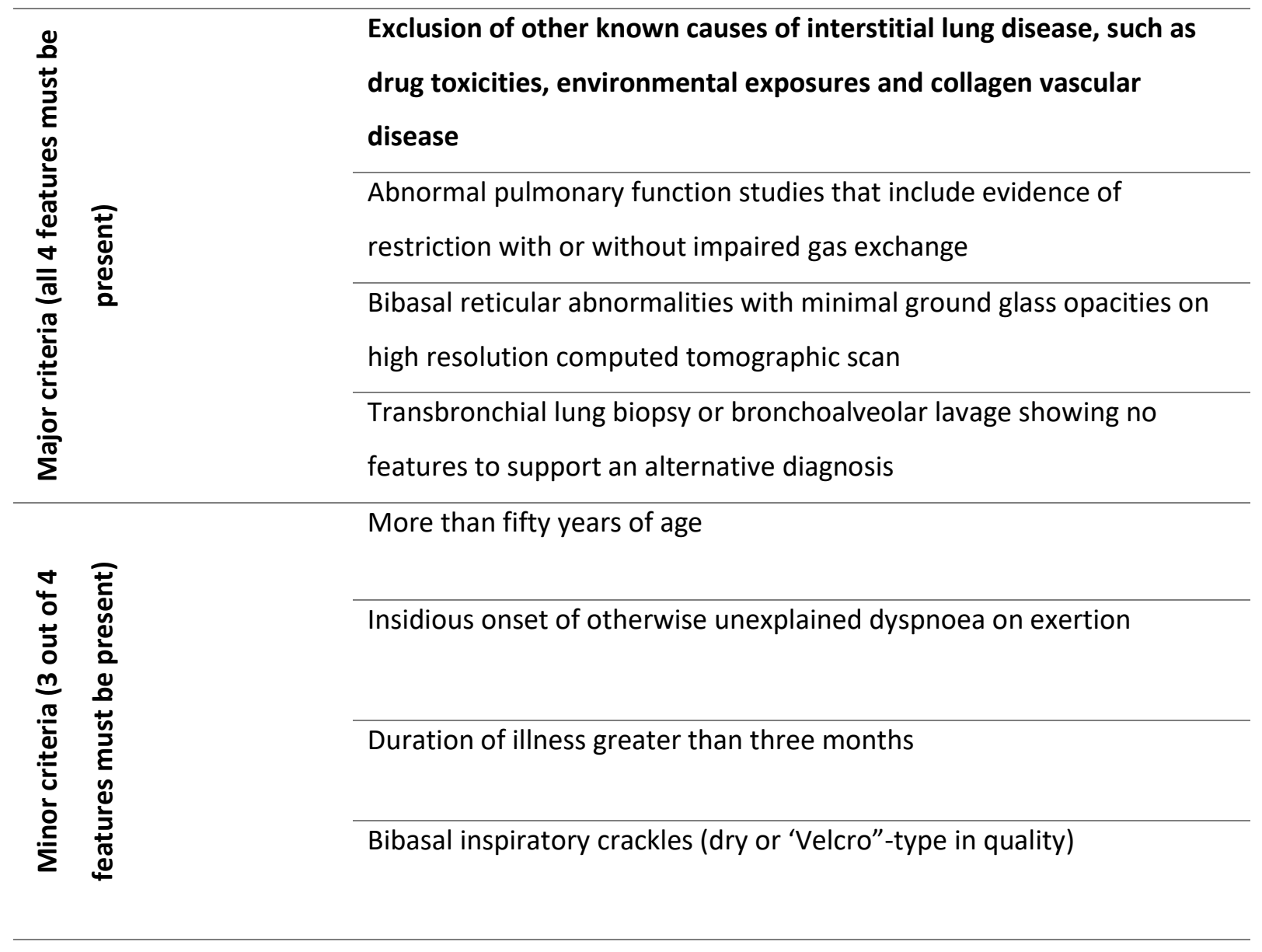

Table 2.1: Diagnostic criteria for IPF without a lung biopsy [9]

\subsubsection{Clinical and Physiological features}

The clinical features of IPF include progressive exertional dyspnoea which can be misdiagnosed as pulmonary oedema or respiratory tract infections, cough and breathlessness [45]. The physiological features of IPF are mainly associated with lung function and can be assessed using spirometry [61]. Spirometry in IPF patients shows an increase in the ratio of forced expiratory volume in 1 second (FEV $)_{1}$ ) to forced vital capacity (FVC) (FEV $1 / F V C)[62]$, reduced total lung capacity (TLC) and a reduction in $\mathrm{FVC} /$ carbon monoxide transfer factor ( $\left.\mathrm{D}_{\llcorner\mathrm{LCO}}\right)$ after 1 year [63], [64].

\subsubsection{Influence on Pulmonary function}

Lung volumes: The results obtained for pulmonary function testing in IPF resembles restrictive impairment of the lung [9]. Residual volume, functional residual capacity (FRC) are reduced in all the patients diagnosed 
with IPF [9]. However, lung volumes in smokers are very high compared with the IPF patients who had never smoked before [65].

Pressure-volume curve: Studies show that the pressure-volume curve is shifted down and right indicating a noncompliant stiff lung [9]. As the disease progresses the lung compliance is further decreased reducing the lung volume further [66].

Airways mechanics: Patients diagnosed with IPF show rapid shallow breaths (referred as tachypneic), hence the work of breathing is high $[9,67,68]$. This is thought to be a mechanical response to a higher elastic load since no chemical basis for hyperventilation is identified $[9,69,70]$.

Gas exchange: Carbon monoxide transfer factor $\left(D_{L C O}\right)$ is reduced due to contraction of the pulmonary capillary volume coupled with ventilation/perfusion abnormalities [9]. A resting arterial blood gas analysis (ABG) of IPF patients shows respiratory alkalosis due to mild hypoxemia caused by ventilation/perfusion mismatch but not by impaired oxygen diffusion or anatomical shunts [9]. During exercise the arterial-alveolar gradient $\left(\mathrm{AaPO}_{2}\right)$ increase by 20 to $30 \%$ and the arterial oxygen saturation $\left(\mathrm{SaO}_{2}\right)$ and arterial oxygen pressure $\left(\mathrm{PaO}_{2}\right)$ falls [9]. Furthermore IPF patients increase their minute ventilation due to an increase in breathing frequency compared to healthy people who increases it by increasing tidal volume $\left(\mathrm{V}_{T}\right)[9]$.

Pulmonary haemodynamics: Pulmonary hypertension is not common during the early onset of the disease [9] but does occur during exercise, even at the early stages of the disease. However when the vital capacity of the lung is below $50 \%$ of predicted values, or the carbon monoxide saturation is below $45 \%$ of predicted, pulmonary hypertension could be expected even during rest [9].

\subsubsection{Management of the disease}

Currently there are limited treatment options for IPF [71]. Various research studies have been conducted in an attempt to eliminate or supress the inflammatory response which was believed to lead to IPF, but with little success [9]. Some of the treatment options includes administering corticosteroids [72,73], cytotoxic and immunosuppressive agents (azathioprine, Cyclophosphamide) $[72,74,75]$ and antifibrotic agents such as colchicine or D-penicillamine $[76,77]$. Whilst new therapies are tested regularly there is no permanent solution in sight other than lung transplant [9].The majority of patients will therefore require supportive care at some point in the course of their disease and national guidelines recommend that oxygen therapy, PR and palliative care should be offered when appropriate [10]. 


\subsection{Pulmonary rehabilitation}

PR programmes are a cornerstone of respiratory patient treatment strategies, providing a programme of exercise, education and support to teach patients how to deal with their condition and maximise their functional capacity. These programmes typically last for a few weeks following the initial diagnosis but the scientific basis underpinning the type, intensity and duration of the exercise component of these programmes remains under-researched [2]. The British Thoracic Society (BTS) published recommendations on creating PR programmes in the UK, based on NICE (National Institute for Health and Care Excellence) guidelines. According to the BTS, PR is an interdisciplinary programme that is tailored to optimise the performance of physical activity and is a key management strategy in people with COPD [10]. The American Thoracic Society states that the primary goal of PR is to "restore patients to the highest possible functional state" [47]. According to the BTS, the following are improved in COPD patients as a result of undertaking PR in adults: exercise capacity, dyspnoea severity, physical activity levels, muscle strength, psychological status, self-efficacy. No such guidelines exist for ILD or IPF patients.

\subsubsection{Constituents of a PR programme}

PR programmes include patient assessment, physical exercise programmes, educational materials, and the promotion of behavioural change, and are designed to improve quality of life [8]. Education is a major component of any rehabilitation programme hence same is said to PR. [BTS guidelines recommends that these sessions should be conducted discreetly and should be aimed to support the lifestyle and behavioural changes to improve self-efficacy. The purpose of education in PR is to support behavioural and lifestyle change and to assist patients in self-management to promote decision making and self-efficacy [10]. These educational sessions are usually based on information about human anatomy/physiology, oxygen therapy, smoking cessation, chest clearance techniques, nutritional advise, goal-setting, confidence-building and selfmanagement [10]. Sewell et al. [78] compared the effectiveness of individualized rehabilitation programmes (personal goal-setting) against general exercise programmes and found no significant difference in the outcomes. The BTS recommends simple generalized training programmes over individualized programmes, and supervised training programmes rather than non-supervised (home based) programmes as part of the rehabilitation process. 


\subsubsection{Duration of a PR programme}

The optimal duration of a PR programme is debatable; although various programmes have been used, lasting for between 4 and 12 weeks, most outpatient PR programmes in the UK last between 6 and 12 weeks [10]. The BTS recommends a 6-12 week programme including a minimum of 12 supervised exercise sessions [10]. Various studies have been conducted to compare the effectiveness of shorter and longer programmes. A study conducted by Green et al. [79] concluded that for patients recruited from the same centre, those undertaking a seven week training programme had better health outcomes than a group who undertook a 4 week programme. Solanes et al. [80] claimed that a minimum of 8 weeks of rehabilitation (with three sessions per week) is necessary for improvements in health outcomes to be observed.

\subsubsection{Physical activity components of PR}

The BTS recommends that a combination of progressive muscle resistance and aerobic training should be included in PR programmes to improve strength and endurance in COPD patients. Aerobic training should focus on improving lower limb endurance such as through walking or cycling [10]. Swallow et al. [81] suggested that because lower limb weakness is common in COPD patients it would be advisable for rehabilitation training to focus on improving leg strength, thereby improving quality of life. Studying a group of 45 COPD patients, Bernard et al. [82] found that those who undertook a combination of strength and aerobic exercise had improved muscle strength but no additional improvement in exercise capacity compared to those who did only aerobic exercise. Mador et al. [83] compared the effects of endurance training alone and combined endurance and strength training in 24 COPD patients. Those who undertook combined training had significant improvements in muscle strength (quadriceps, hamstrings, pectoralis major and latissimus dorsi) but no significant improvement in quality of life compared with the group who did endurance exercise alone. In contrast, Vonbank's study of 36 COPD patients found that those who took undertook progressive strength training alone showed improved muscle strength, exercise capacity and quality of life compared with those who undertook either endurance training alone or a combination of strength and endurance training [84].

\subsubsection{Compliance with a PR programme: barriers and facilitators}

Particular attention should be given to minimise the dropout rate in PR programmes. There are four main reasons why people drop out from these programmes [85]: (1) the intensity of the programme being perceived as too high, (2) transportation difficulties, (3) failure to perceive health improvements, and (4) unsupportive families or friends. Bjoernshave et al. [86] suggested that other reasons include not feeling comfortable in taking 
part in group activities, not feeling safe with health professionals who are conducting rehabilitation programmes, and personal beliefs or experiences about their illness. These authors stated that good support from family and peers would enhance patient adherence to PR.

It is important to talk to patients before enrolling them in a PR programme [10], to consider their individual perceptions, needs and experiences [87]. This is perhaps especially pertinent for IPF patients who, according to Duck et al. [87], perceive three main difficulties: issues with diagnosis, reduction in quality of life and issues concerned with living with the condition. Commenting on the experiences of IPF patients in the UK, Bajwah et al. [88] indicated that a typically difficult and protracted diagnosis is usually followed by a further stressful period for these patients. This is mainly due to the lack of information they receive about IPF and about their prognosis, which is likely one of the main confounders to attempts to improve the quality of life of these patients [88]. Bajwah et al. [88] also identified that early referral to palliative care (PR) and support groups, as well as providing patients with psychological support, would improve the quality of life in IPF patients.

\subsubsection{Pulmonary rehabilitation in ILD and IPF}

Although PR is a well-established method to treat patients with COPD to improve their exercise performance [8], the same cannot be said for ILD patients. There are very little physiological data reported from well-defined studies of the effectiveness of PR in managing patients with IPF [9]. The ATS recommend that a PR programme for ILD patients should consist of a combination of exercise training, education, and psychological support in order to improve exercise tolerance, reduce the symptoms of breathlessness, and ultimately to improve quality of life $[9,91]$.

A study conducted by Swell et al. [78] randomised 180 patients between a control group which followed an individualized training programme and the study group who followed a group exercise session. The individualized training programme had individualized exercises identified through the Canadian Occupational Performance Measure (COPM) while the test group followed a set of 10 general exercises. The study showed that groups had improved their fitness similarly (no significant difference).

Studies conducted by Kozu et al. compared the responses of IPF and COPD patients to PR. 45 IPF and 45 COPD patients were assessed, and the study showed that the improvement achieved in IPF patients (for all outcome measures) were less than in COPD patients. The existing literature on this subject area is unclear about the outcome of PR programmes on ILDs [13]. Nishiyama et al. [94] evaluated the effects of PR in 13 IPF patients, finding no statistically significant influences on measures of pulmonary function, arterial blood gas analysis or 
dyspnoea ratings. However, six-minute walk test distances and health-related quality of life scores (St George's quality of life questionnaire) showed marked improvements [94]. Another study conducted by Jeffrey at al. claimed that PR improves functional capacity and fatigue levels in IPF patients [95]. Holland et al. [96] found improvements in six-minute walk test distance, forced vital capacity and dyspnoea in 25 ILD patients following 8 weeks of PR. Similar results were obtained by Ozalevil et al. [97] in which a home-based PR programme reduced perceived dyspnoea and leg fatigue and improved the six-minute walk test distance.

Although PR appears to have some positive effects on IPF patients, this improvement could be dependent on the severity of the disease. Kozu et al. [92] assessed the influence of PR in 65 IPF patients, concluding that IPF patients with a lower dyspnoea score responded better to PR than those with a higher dyspnoea score. Furthermore the biggest issue with PR for IPF patients is that any initial improvements are not sustainable over a long period of time [98]. This was confirmed by Spiruit et al. [8] who stated that PR has little effect on IPF compared to COPD and improvement is not sustained for a period longer than six months. Holland et al [15]. observed 34 IPF patients who underwent PR for eight weeks, again finding that initial improvements in the sixminute walk test distance, dyspnoea scale and level of fatigue were not sustained at six months following intervention.

Therefore, whilst there is some evidence of benefit from PR in IPF, relatively little is understood about its influence on either oxygen uptake, delivery or utilisation capacity or its efficiency in these individuals.

\subsection{Evaluating the physiological impact of Pulmonary Rehabilitation}

\subsubsection{Heart rate variability and heart rate dynamics}

Heart rate dynamics are thought to provide a good proxy for the response of cardiac output and muscle blood flow to increased metabolic demand [27], albeit they do not account for the contribution of stroke volume in meeting this demand. One way to determine HR responsiveness is to measure the variation in beat-to-beat duration of the cardiac cycle, known as HRV [99]. This technique has been widely used to estimate the influences of the autonomic nervous system (ANS) on heart rate in various populations. Physiological factors including blood pressure and respiratory rate have a profound effect on the autonomic modulation of heart rate in healthy people. HRV analysis provides a non-invasive method for estimating the magnitude and temporal variation of these influences. Unusual magnitudes or patterns of variation of HRV can indicate possible autonomic dysfunction. 
Two main categories of statistical indices have been used to quantify HRV: i) the time domain measures and ii) the frequency domain measures. Time domain analysis is conducted using descriptive statistical methods, and two indices are commonly used: i) 'SDNN' is the standard deviations of all 'normal-to-normal' RR intervals; it provides an overall estimation of HRV (including the influences of both sympathetic and parasympathetic nervous systems) [28]; ii) 'RMSSDNN' is the square root of the mean squared difference of successive RR intervals; it quantifies the high frequency components of the RR time series and so reflects the activity of the parasympathetic nervous system [28].

Frequency domain analysis involves splitting the heart rate signals into different frequency components, allowing analysis of the relative power of the constituent frequency 'bands' [100]. By analysing these components, it is possible to understand the relative influences of the sympathetic and parasympathetic components of the ANS on heart rate (or, more correctly, on cardiac interval). One common method of calculating the power distribution of a signal is through power spectral density (PSD) analysis. PSD quantifies the power of a signal within a very narrow frequency range; in HRV analysis the PSD is integrated across specified frequency bandwidths [100] to quantify the sympathetic and parasympathetic components of the HRV data. A graphical representation of the power distribution of cardiac interval (RR) data is shown in Figure 2.1. The plots indicate that three different frequency ranges contribute to the RR data: 1) High frequency components (HF) in the range $0.15-0.40 \mathrm{~Hz}, 2$ ) Low frequency components (LF) in the range $0.04-0.15 \mathrm{~Hz}$, and 3) Very low frequency components (VLF) in the range $\leq 0.04 \mathrm{~Hz}$ ) [100]. VLF is associated with thermoregulation and vasomotor tone, LF activity is associated with the baroreceptor (blood pressure regulatory) reflex, and HF activity is associated with respiration [100], [101]. Figure 2.2 illustrates the averaged power distribution during periods of rest, exercise and recovery for the same individual. 


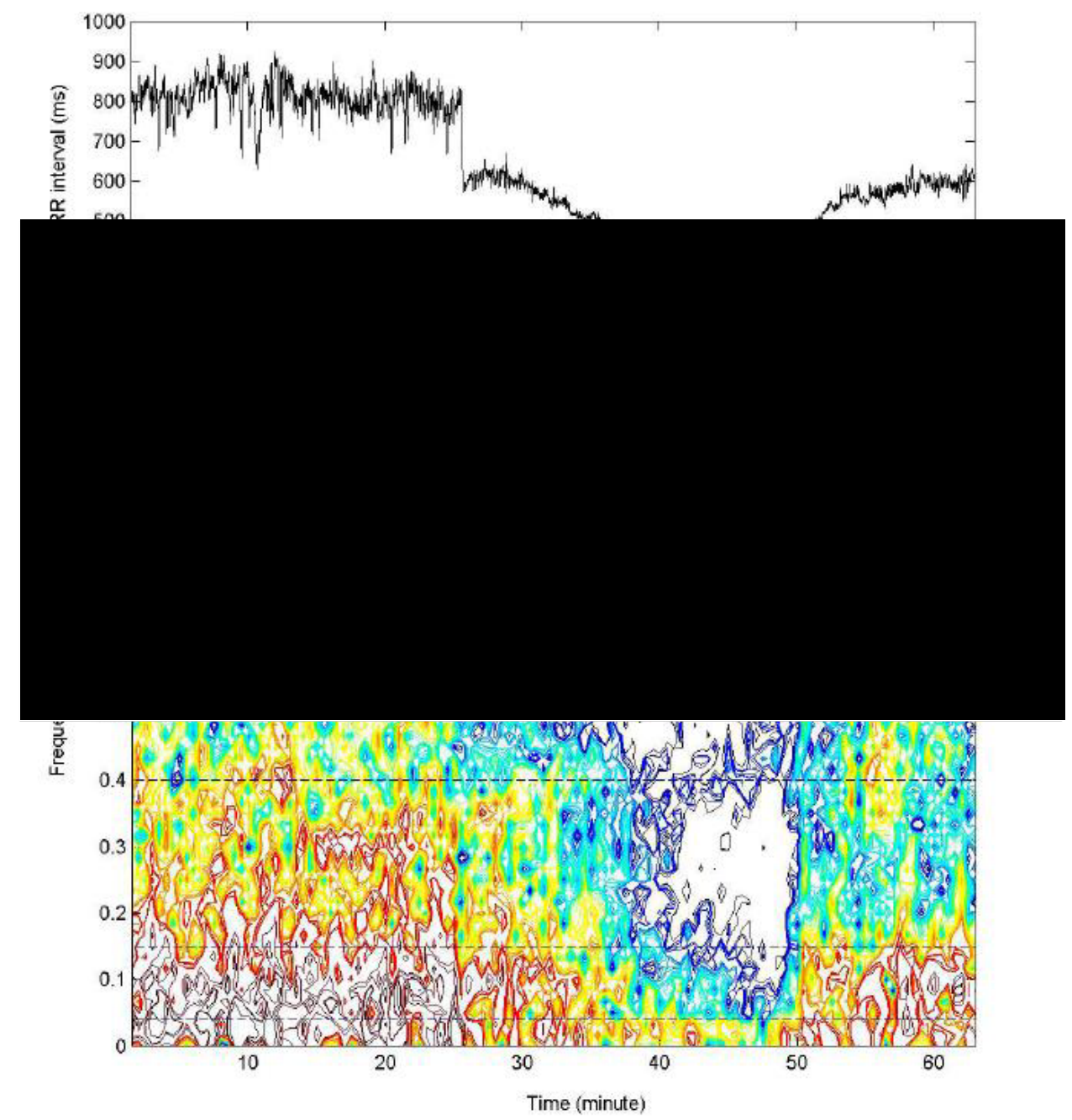

Figure 2.1: (top) RR interval of a healthy 21-year-old male during rest (0-24 $\mathrm{min})$, exercise (24-48 $\mathrm{min})$ and recovery (48-63 min). (bottom): time-frequency plot for the same individual as above. High power is represented by red, medium power by orange/yellow, and low power by blue [100]. 


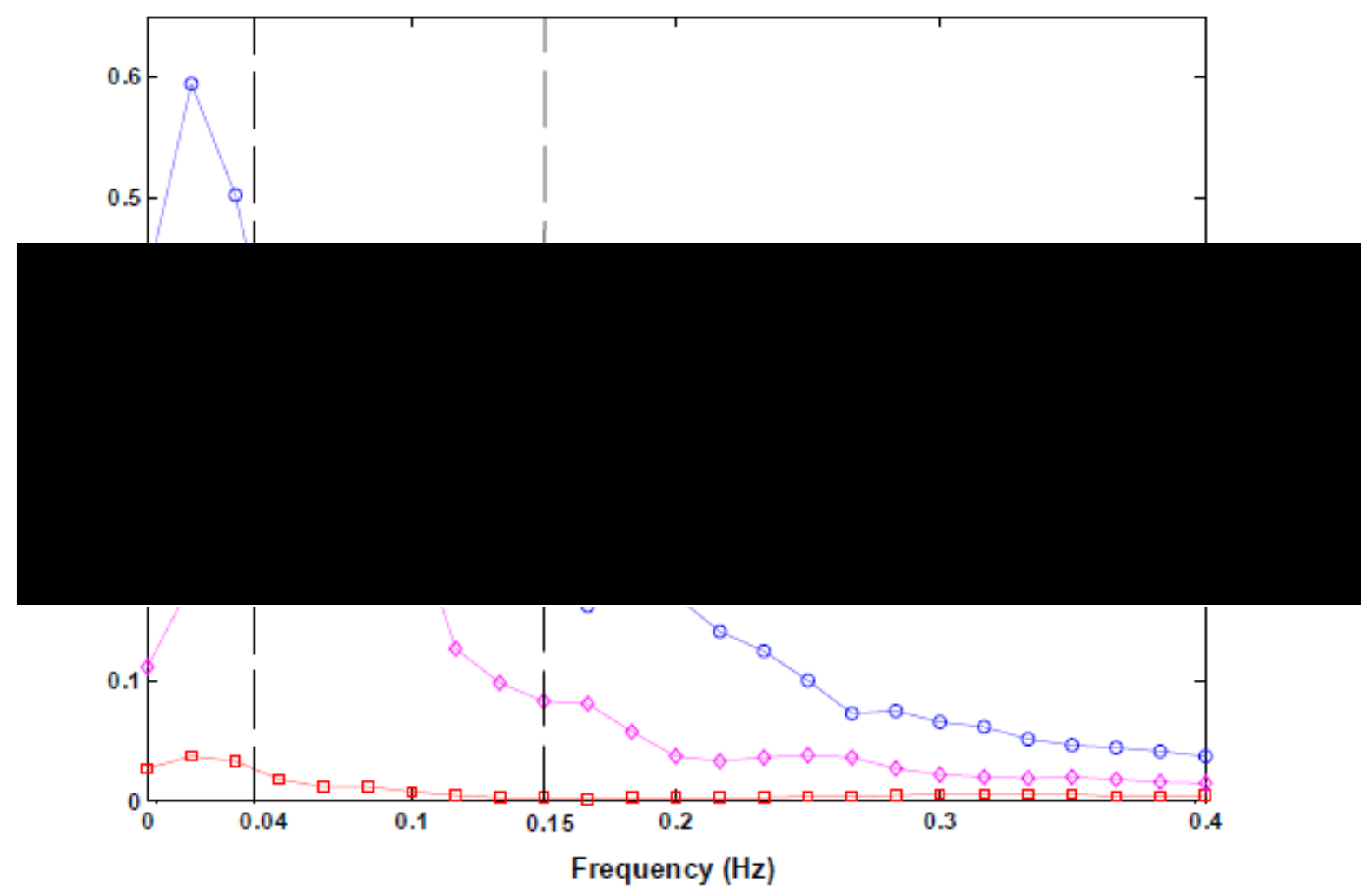

Figure 2.2: Power spectrum for periods of rest, exercise and recovery for the same individual as in Figure 2.1 [100].

$\mathrm{HRV}$ can be used across a range of pathologies since it is a non-invasive and relatively easy way to measure the activity of the ANS on the heart. Despite its advantages, caution must be applied when HRV is used to determine an individual's capacity to increase or decrease heart rate. In particular, HRV must be determined during steadystate conditions and for extended periods, so it cannot provide an overall measure of the range of cardiac dynamics that occur across multiple states or that are transient in nature. An alternative measure of cardiac dynamics that avoids these restrictions uses the technique of PRSA [29]. This method separately characterizes all heart rate modulations that result in either accelerations or decelerations within an RR data set. The PRSA procedure involves aligning sections of the RR data series relative to selected 'anchor points', which correspond to either points of increase in the signal (deceleration) or of decrease in the signal (acceleration) [29]. Signal averaging of the two phase rectified data sets then enables quantification of the 'acceleration capacity' (AC) and 'deceleration capacity' (DC) for the individual [102]. PRSA is a good indicator of the vagal activity of the heart [102] (AC and DC reflect the magnitude of reduced (vagolytic) or enhanced (vagotonic) parasympathetic (vagal) modulation of cardiac interval, respectively). Bauer et al. [103] concluded that DC is a good indicator of 
mortality after myocardial infarction (MI) and that it is more accurate than left ventricular ejection fraction (LVEF) and HRV, whilst Kantelhardt et al. [104] found that survivors of myocardial infarction showed a linear decrease in DC with age.

It is known that individuals with COPD have a degree of cardiac autonomic dysfunction, reflected by altered HRV during rest and exercise [30] but with a normal diurnal variation [31]. They also have a tendency towards reduced PRSA-derived cardiac dynamic capacities that are ameliorated with oxygen therapy [105]. However, as far as we are aware, neither HRV nor cardiac acceleration/deceleration capacities have been evaluated in individuals with IPF. A comparison of these variables before and after physical rehabilitation might indicate whether they would benefit from such interventions in terms of improved cardiac responsiveness to everyday activities.

\subsubsection{Oxygen uptake kinetics and oxygen uptake efficiency slope}

Maximal oxygen uptake is considered to be the single best measure of aerobic fitness (capacity for oxygen utilisation) but in practice its measurement can be difficult (for example in older people or those with cardiorespiratory disease state, who have limited functional capacity). Other traditional measures of aerobic exercise tolerance such as $\dot{\mathrm{VO}}_{2}$ at the ventilatory anaerobic threshold (VAT) can be similarly problematic in older/patient populations. Alternative techniques have been developed that offer advantages in terms of exercise tolerance. Two methods are of particular note: 1) 'Pulmonary oxygen uptake kinetics' analysis, and 2) 'oxygen uptake efficiency slope' analysis.

Pulmonary oxygen uptake kinetics analysis involves mathematical modelling of the rate of change of oxygen utilisation in response to a sudden change in exercise workload. This provides insight into an individual's ability to absorb and utilize oxygen to fuel metabolic activity [23]. The procedure involves fitting either a monoexponential or bi-exponential model to the empirical temporal data, with the time-constant of the best-fit model reflecting an individual's oxygen utilisation response rate. Several studies have explored the influence of physical PR on oxygen uptake kinetics in COPD [8]. Puente-Maestu et al [106] examined the effects of supervised and unsupervised eight-week PR on a mono-exponential oxygen uptake model, observing a post-PR increase in mean endurance time ( $493 \pm 281$ seconds in the supervised group and $254 \pm 283$ seconds in the unsupervised group) when participants exercised at a work rate equivalent to $70 \%$ of $\mathrm{VO}_{2}$ max. Furthermore, these authors also observed that mean $\mathrm{Tau}(\tau) \dot{\mathrm{V}} \mathrm{O}_{2}$ decreased from $83 \pm 17$ to $67 \pm 11$ seconds (supervised group) and from 84 \pm 12 to $79 \pm 16$ seconds (unsupervised) for the two groups. Mean $\tau$ for $\mathrm{CO}_{2}$ output, minute ventilation and heart rate were also observed to have increased post-training. Similar studies in IPF patients have been limited. McNarry et al. [107] observed that the peak rate of oxygen uptake $\left(\mathrm{V}_{2}\right)$ in IPF patients is half that of similarly 
aged healthy individuals, whilst their peak heart rates are $20 \%$ lower and their kinetic HR responses to heavy intensity exercise are 2.5 times slower. These results provide evidence of reduced tissue oxygen utilization following the onset of exercise in people with IPF, which in large part results from impeded central (cardiovascular) oxygen delivery. However, the study included only three IPF patients. Jackson et al. [108] observed that a three-month PR improved treadmill exercise time and maintained oxygen consumption $\left(\mathrm{V}_{2}\right)$ in 11 IPF patients following rehabilitation, whereas a control group suffered significant reduction in $\mathrm{VO}_{2}$ over the same period.

Baba et al. [17] introduced the 'OUES', which quantifies the body's rate of increase of oxygen uptake $\left(\mathrm{VO}_{2}\right)$ in response to increases in minute ventilation (VE) during incremental exercise. OUES is calculated using the linear relationship between $\mathrm{VO}_{2}$ and the logarithm of VE during incremental exercise:

$$
\dot{\mathrm{V}} \mathrm{O}_{2}=\mathrm{a} \times \log 10 \mathrm{VE}+\mathrm{b}
$$

where "a" (the slope or the OUES) represents the rate of increase of $\mathrm{VO}_{2}$ in relation to VE and " $\mathrm{b}$ " is the intercept $[18,19] . \mathrm{VO}_{2}$ is graphically presented on the $y$-axis and the logarithm of VE on the $x$-axis, with a greater slope reflecting greater efficiency [19]. Furthermore, use of the logarithm of VE linearizes the otherwise curvilinear $\grave{\mathrm{VO}}_{2} / \mathrm{VE}$ slope, leading to a patient effort-independent result [19]. This is especially useful when using the method with individuals who have cardio-respiratory dysfunction (as in chronic lung disease), who have limited capacity to conduct strenuous exercise. The relationship between $\mathrm{VE}$ and $\mathrm{V}_{2}$ is determined by the pulmonary dead-space/tidal volume ratio $(\mathrm{Vd} / \mathrm{Vt})$, the arterial partial pressure of carbon dioxide $\left(\mathrm{pCO}_{2}\right)$, and the respiratory exchange ratio (RER). Consequently, OUES facilitates a mechanistic assessment of two fundamental cardiovascular processes during exercise: 1) blood perfusion to the lungs (related to $\mathrm{Vd} / \mathrm{Vt}$ ) and 2) blood perfusion to working muscles (related to $\mathrm{pCO}_{2}$ and $\mathrm{RER}$ ), in response to underlying metabolic demand. OUES appears to have several advantages compared with other measures of aerobic exercise performance: it can be measured accurately from submaximal exercise protocols (below 60-75\% functional reserve) ] [2, 8, 14, 16, 25, 109] yet is strongly correlated with $\dot{\mathrm{V}}_{2} \max [2]$; it is completely objective (tester-independent) [2, 8, 109]; it has better inter-protocol agreement [10]. OUES has been quantified in various populations (children and adults with heart disease, elite athletes $[4,109])$ but not in patients with chronic lung disease. 


\subsection{Evaluating Psychological, sociological and physical impact of the disease on quality of life}

\subsubsection{Interpretive frameworks}

There are two theories used when it comes to evaluation of psychological impact of a disease on an individual. They are 1) Deci and Ryan's [111] self-determination theory and 2) Lazarus and Folkman's [113] transactional theory of stress and coping. In brief, self-determination theory (SDT) is a theory of human motivation and personality in the social context; it is further explained by six mini-theories, one of which considers the role of basic psychological needs in determining our well-being and positive functioning. These include the need to feel competent, related, and autonomous [111]. When need-satisfaction is thwarted our well-being and functioning are adversely affected [111]. Competence refers to the need of an individual to be effective and capable in their social environment [114]. Relatedness describes the need of an individual to relate to, interact with, and experience the care of, others, and autonomy refers to the need to be independent and able to control oneself and one's life. These three psychological needs are universal and their fulfilment leads to psychological wellbeing, which is reflected in physical well-being and vice versa [111] [112]. The usefulness of these theories to explore chronic health conditions has been demonstrated previously. For instance, Rahman et al. [115] used SDT to analyse motivation and need satisfaction as predictors of psychological and behavioural outcomes in patients presenting with a range of health conditions who engaged in an exercise referral scheme. A further study by these authors involving cardiac rehabilitation patients reinforced the value of SDT for understanding the experiences of patients who are suffering from chronic conditions [116].

Lazarus and Folkman propose that stress is not simply an emotion but also the outcome of an ongoing relationship between the individual and their environment [112] [113]. More specifically, when an individual encounters a stressor (such as an IPF diagnosis) they will evaluate the impact of the stressor in relation to personal objectives in their life. This process is termed appraisal, of which there are four possible evaluations: Harm/Loss (referring to damage already caused, e.g. being diagnosed with a terminal disease), Threat (referring to potential harm, e.g. an upcoming medical test), Challenge (the event offers opportunity for personal growth/achievement) and Benign (the event is not stressful and no action is taken) [112]. These appraisals are not mutually independent and could occur simultaneously at any given time when faced with a stressor. Lazarus states that coping is a cognitive and behavioural effort employed by a person to tackle demands that are created by the stressors [117] [113]. He further theorises that different stressors will lead to the use of different coping mechanisms (problem-focused and emotion-focused). Problem-focused coping mainly aims to resolve the practical problems associated with the stressor on a daily basis. These include changing lifestyle through seeking information on the stressor, or planning and prioritizing [117]. Emotion-focused coping revolves around 
managing unpleasant feelings that are caused by a stressor - for example isolation, distancing, managing hostile feelings and wishful thinking [117] [118]. 


\section{Chapter 3}

\section{Methods}

The studies reported in this thesis consist of qualitative and quantitative investigations focussing on patients with IPF. The first is a qualitative assessment of patients' experiences of coping with this disease and, based on this experience, their recommendations for its clinical management. The second is a set of assessments of the physiological impact of a PR programme in IPF patients. This chapter describes the methods used in the design and execution of each study.

\subsection{Study 1: Patients' experiences of coping with Idiopathic Pulmonary Fibrosis and their recommendations for its clinical management}

\subsubsection{Study design and setting}

The aim of this first study was to understand how patients coped with their initial diagnosis, how they live with the disease day-to-day, their experiences and opinions of the professional support they received, and their views on any rehabilitation support that they were offered. A patient-centred approach was therefore used to explore the social, psychological and physical impact of IPF. Semi-structured interviews were conducted by an experienced academic. Interview questions were written by the researchers but guided by informal conversations with patients. An inductive thematic approach was used to analyse the data, allowing us to identify common themes in the patients' experiences. Ethical approval to conduct the study was obtained from the College of Engineering Research Ethics Committee (Appendix A), and the study was performed in accordance with the Declaration of Helsinki.

\subsubsection{Participants}

We recruited participants from a monthly support group for patients with IPF in Swansea. We first contacted the chairman of the support group to gain his approval to approach group members. Members of the research team then attended a group meeting to provide an overview of the study; patients had an opportunity to ask questions and were then invited to participate in the study. Those interested in participating were then provided with patient information sheets and contacted via email or telephone to arrange individual interviews at convenient locations. This was followed by a second round of participant recruitment (via invitation letters posted to members of the group), and similar arrangements were made regarding interviews. Our sampling criteria required that participants must have been diagnosed with IPF, be aged 18 years or older and be able to write and converse in English (to facilitate data collection). 


\subsubsection{Qualitative data collection methods}

All interviews were conducted by two individuals: i) a senior member of the research team who had extensive experience of conducting qualitative research, but who had little knowledge of IPF prior to conducting the interviews, and ii) the primary researcher (SS). The senior researcher helped to reduce bias in the interview process, and as she was an experienced participant interviewer it ensured that patients were put at their ease (from their detailed and in-depth responses, participants appeared to be very comfortable discussing the personal aspects of their disease). The primary researcher was knowledgeable about IPF and about PR, so took the lead on asking participants specific questions about the rehabilitation programme and its role in disease management.

At the outset of each interview, participants were reminded of the purpose of the study, that participation was entirely voluntary and that they were free to stop the interviews at any point and to decline to answer any question if they wished. Once any queries were addressed, they were asked to provide their written informed consent to participate. The interviews were semi-structured, following the steps outlined by Rubin and Rubin [123]. The interviews started with introductory questions, followed by transition questions, then the main questions and summary questions. An exact list of questions asked in this study cannot be provided as the specific questions posed were led by the participants' responses; however, an overview and indication of the purpose of the intended questions which guided the interviews is given in Table 3.1. The questions were devised by the two interviewers based on the primary researcher's knowledge of IPF and prior interactions with patients in the support group and with the clinical staff treating them. Interviews lasted for approximately 45-60 minutes and were audio recorded to assist with transcription at a later date. The interviews were transcribed verbatim. To maintain anonymity, pseudonyms were given to individual participants when reporting their responses, and all names and locations were redacted from the transcripts. (After the first interview was transcribed, the interviewers reflected on the interview experience and the responses yielded. This resulted in minor changes to the wording of the opening questions to ensure optimal focus on the main topic of subsequent interviews.) 
Table 3.1: Example of the Overview and the purpose of questions asked during patient interviews.

\begin{tabular}{|c|c|c|}
\hline Question type & Example question & Purpose of the question \\
\hline Introduction & $\begin{array}{l}\text { Can you tell me about the onset of } \\
\text { your condition and a little bit about } \\
\text { it? }\end{array}$ & $\begin{array}{l}\text { This question aimed to engage the patient with the } \\
\text { topic and to allow them to express their personal } \\
\text { understanding of their condition. }\end{array}$ \\
\hline Transition & $\begin{array}{l}\text { How does IPF make you feel on a } \\
\text { day-to-day basis? }\end{array}$ & $\begin{array}{l}\text { This question was used to gain an overall } \\
\text { impression of the psychological impact of the } \\
\text { disease from the patient's perspective, and to link } \\
\text { the introductory and main questions. }\end{array}$ \\
\hline Transition & How do you make the most of it? & $\begin{array}{l}\text { These questions helped to facilitate the flow } \\
\text { between topics of interest during the interview. } \\
\text { These types of question were followed by a main } \\
\text { question, depending on the patient's response. }\end{array}$ \\
\hline Main & $\begin{array}{l}\text { If you think back to your life before } \\
\text { being diagnosed with your medical } \\
\text { condition, what are the things that } \\
\text { you did before but are prevented } \\
\text { from doing now? }\end{array}$ & $\begin{array}{l}\text { This type of question was asked to get an indication } \\
\text { of the patient's lifestyle before diagnosis of the } \\
\text { disease and how IPF had affected the patient's daily } \\
\text { life. This is the key information in which the } \\
\text { interviewers were interested. }\end{array}$ \\
\hline Summary & $\begin{array}{l}\text { Is there any other way that it } \\
\text { affects you? }\end{array}$ & $\begin{array}{l}\text { These questions were intended to sum up the main } \\
\text { topics of the interview, allowing the patient to } \\
\text { summarise their overall experiences and personal } \\
\text { perspectives of living with the disease. }\end{array}$ \\
\hline
\end{tabular}

\subsubsection{Data analysis}

As this is an under-explored area, an inductive thematic approach [124] was used to analyse the data, allowing identification of common themes representing the patients' experiences and also helping us to relate their personal stories. Using this approach, notes and initial impressions (raw data themes) were recorded during the interviews and these ideas were tracked and continually revised throughout the transcription process. This analysis followed strategies outlined by Miles and Huberman [125] where quotes (representing raw data) were highlighted and labelled with an initial raw data theme that reflected the idea or meaning conveyed in the quote. This process continued until all quotes relevant to the research aim were highlighted and either allocated to an existing raw data theme or, if they did not fit into an existing theme, given a new raw data theme label. To ensure that the original meaning of the raw data was retained, following this initial process, the primary 
researcher reassessed the transcripts to verify the allocations of labels to the raw data. The raw data generated by interview quotes were coded using patient identification codes to allow us to assess how representative each theme was of overall participants' experiences. After the data were coded and categorized into raw data themes, they were reviewed systematically to identify common meaning across different raw data themes. Those that had shared common meaning were grouped together to form a 'first-order theme', which was given an appropriate label to represent the shared common meaning of its composite raw data themes. This process was repeated with the resulting first-order themes to produce 'second-order themes', and finally with the second-order themes to produce 'general dimensions.

An extended process of peer-debriefing was used to establish the trustworthiness of the data analysis: the senior interviewer acted as a critical friend to challenge and check the credibility of the data analysis and interpretation (performed by SS). The critical friend independently reviewed each transcript and highlighted salient quotes to produce raw data. Based on the classifications by SS, she then determined whether she was in agreement with their allocation to raw data themes and noted where her allocations differed. She also noted where she identified additional raw data and to which themes, she would allocate these. The two analysts discussed this first stage of analysis and agreed on some changes to the allocations of raw data to raw data themes, and on the addition of new raw data to some themes. The analysts then discussed the initial identification of first and second-order themes. SS independently reviewed the modifications discussed during peer-debriefing and incorporated those he felt were appropriate into the next interpretation of the data. 'Member checking' was also used, although only to a limited extent as only two participants engaged with this process. This involved forwarding interview transcripts to participants for review prior to data analysis. Those who responded verified the transcript content and none replied asking to withdraw their comments or objecting to their interview content.

\subsection{Study 2: Physiological impact of pulmonary rehabilitation for IPF patients}

\subsubsection{Study setting}

This part of the study was also conducted in accordance with the Declaration of Helsinki. Ethical approval to conduct the study was obtained from Wales Research Ethics Committee 6 (Ref. 16/WA/0272; Appendix A), part of the UK Health Departments' Research Ethics Service. Approval and permission to execute the study was obtained from the local health board (Abertawe Bro Morgannwg University Health Board) (Ref. 206031; Appendix A). Swansea University was the Sponsor for the study, as required by the Research Governance Framework for Health and Social Care and/or Medicines for Human Use (Clinical Trials) Regulations 2004. 


\subsubsection{Participants}

Participant recruitment was led by a consultant chest physician, who informed eligible patients about the study (including its purpose, protocol, expected commitment and the possible risks of involvement). Patients attending the Interstitial Lung Disease Clinic at Moriston Hospital, Swansea, who had been diagnosed with IPF by a Multi-Disciplinary Team, according to the American Thoracic Society (ATS) and European Respiratory Society (ERS) guidelines [2] were potentially eligible to participate in the study. An additional clinical assessment was performed on all potential participants; they were not eligible to participate if they had any of the following exclusion criteria:

- Respiratory infection within the previous two months

- Known coronary or valvular heart disease

- Any other serious co-morbid condition (e.g. emphysema, uncontrolled diabetes, renal disease)

○ Significant hypoxia (oxygen saturation below 85\%) when breathing air and undergoing conventional screening for a PR programme

- Musculoskeletal problems that would make it impossible to exercise

- Current smokers.

All recruited participants provided their written informed consent to participate in the study. They were then enrolled in the PR programme (Section 3.2.4) at Morriston Hospital, Swansea.

\subsubsection{Physiological data collection methods: Pre- and post-rehabilitation testing}

\subsubsection{Day 1}

Participants were first asked to complete the Quality of Life questionnaires (European Quality of Life -5 Dimensions (EQ5D) questionnaire developed by the EuroQol Group [126], Hospital Anxiety and Depression Score (HADS) [127], MRC breathless scale [128] and Kings ILD questionnaire [129]). This was followed by the six-minute walk test (6MWT). The 6MWT evaluates the integrated response of all the systems involved during exercise, especially the pulmonary and cardiovascular system [130]. The tests were performed according to the American Thoracic Society's guidelines [131]. Two cones were placed on either end of a 30 meters corridor to mark the test 'course'. Participants were then asked to walk between the cones as fast as they could for six minutes, and the distance covered was recorded. Each of the above assessments was carried out at Morriston Hospital, Swansea. The results of this study are reported in Chapter 4. 


\subsubsection{Day 2}

Participants were asked to visit the lung function laboratory at Morriston Hospital, Swansea, for baseline physiological assessment. They were asked to not consume alcohol for 24 hours before the test day and to not consume caffeine for six hours prior to the scheduled tests, but otherwise to have eaten and consumed fluids as normal. To test the cardiovascular and the respiratory systems, participants were asked to complete an incremental exercise test on a stationary bicycle (ergometer) (VIAsprint ${ }^{\mathrm{TM}}$ 150P Ergometer, Vyaire Medical, Mettawa, IL, USA) in accordance with methods described by Arena et al. [132]. They first completed three minutes of baseline cycling with no workload, after which the work rate was increased at a rate of $10-15 \mathrm{~W} \cdot \mathrm{min}^{-}$

${ }^{1}$ depending on the individual predicted maximum workload, until the limit of exercise tolerance was reached. Participants were asked to maintain a cadence of 55-60 rpm. The test was stopped by the researchers if a patient desaturated to an oxygen level below $85 \%$. Throughout the test, which included six-minute periods of rest before and after exercise, a continuous Holter ECG was recorded (Reynolds Lifecard Digital CF system; Spacelabs Healthcare Ltd., Hertford, UK), providing ECG data with a $1024 \mathrm{~Hz}$ sampling frequency, 12-bit resolution and a sample accuracy of $2.5 \mu \mathrm{V}$. The ECG leads were positioned in the 'modified V5, CC5, modified $V 5 R^{\prime}$ electrode configuration. Patients were also asked to breathe through a facemask which allowed us to sample the air that they inspire/expire using an online respiratory gas analyser (MGC Ultima CardiO2; MGC Diagnostics, St Paul, MN, USA) and blood oxygen saturation was continuously monitored (Nonin Model 7500, Plymouth, MN, USA). The rates of oxygen uptake $\left(\mathrm{V}_{2}\right)$ and carbon dioxide production $\left(\dot{\mathrm{V}} \mathrm{CO}_{2}\right)$ and minute ventilation (VE) were measured from the breath-by-breath respiratory gas data using the Breeze computer software (Breeze Software 6.4.1, Medical Graphics, MN, USA). The $\dot{\mathrm{VO}}_{2}$ maxwas determined by averaging the $\grave{\mathrm{V}}_{2}$ values from the final 10 seconds before the limit of exercise was reached. Aerobic threshold (AT) was identified by two physiologists using a combination of the two methods used by Castro et al. [133]: (1) using the point of upward inflection of the $\dot{\mathrm{VCO}}_{2} \mathrm{vs}_{\mathrm{VO}_{2}}$ curve and (2) using the onset of a consistent increase in ventilatory equivalent for oxygen $\left(\mathrm{VE} / \mathrm{VO}_{2}\right)$ with no increase in the ventilatory equivalent for carbon dioxide $\left(\mathrm{VE} / \mathrm{V} \mathrm{CO}_{2}\right)$. Forced expiratory volume in one second (FEV1) and forced vital capacity (FVC) were measured using the spirometry test function of the online respiratory gas analyser. All tests were initiated at the same time of day ( \pm 1 hour). The results of this study are reported in Chapter 5 .

\subsubsection{Day 3}

Participants returned to the lung function laboratory at Morriston Hospital, Swansea, having again refrained from consuming alcohol for 24 hours from consuming caffeine for six hours, but to have eaten and consumed 
fluids as normal. Participants were asked to perform a second bicycle exercise test (VIAsprint ${ }^{\mathrm{TM}}$ 150P Ergometer, Vyaire Medical, Mettawa, IL, USA) consisting of a six-minute warmup with no external resistance, followed by an immediate transition to a fixed uniform workload. The uniform workload was calculated as $40 \%$ of the difference between the AT and the $\dot{V}_{2 M a x}(\Delta 40 \%)$ [16] which was calculated from the incremental test performed on day 2. This workload was maintained for six minutes or until the participant reached their limit of tolerance, after which there was an immediate transition back to cycling with no external resistance for a further three minutes. Participants were asked to maintain a pedalling cadence of 55-60 rpm throughout each stage of the test. Patients breathed through a facemask connected to an online respiratory gas analyser (MGC Ultima CardiO2; MGC Diagnostics, St Paul, MN, USA) and a Holter ECG recorder (Reynolds Lifecard Digital CF system; Spacelabs Healthcare Ltd., Hertford, UK) recorded an ECG throughout the test. All tests were initiated at the same time of day ( \pm 1 hour). The results of this study are reported in Chapter 6 .

\subsubsection{The Pulmonary Rehabilitation programme}

Following baseline measurements participants began an eight-week PR programme (two sessions per week, two hours per session) under the direction of the physiotherapist staff at Moriston Hospital, Swansea. This programme consisted of ten minutes of 'warming up' (20 sets of 'marching on the spot', 'heal digs', 'knee lifts', 'shoulder rolls', 'knee bends' and 'high knees') followed by a range of strength training exercises ('bicep curls', 'triceps extension', 'dumbbell press', 'dumbbell fly', 'leg extension' and 'dumbbell squats') and cardiovascular exercises (choice of treadmill, bicycle ergometer or cross-trainer exercise). Each patient was asked to choose a weight that they could safely handle at the beginning of the rehabilitation for use in each exercise, and they were encouraged to increase their weights on each day of rehabilitation as the programme progressed. For the cardiovascular exercise patients were asked to select a mode of exercise with which they were comfortable, which was maintained throughout the programme but with increasing workload (resistance) as rehabilitation progressed. The strength exercises lasted five minutes each and the cardiovascular exercise lasted ten minutes, followed by a ten-minute cool-down period (which included sets of 'hip flexor stretch', 'thigh stretch', 'calf stretch' and 'lower back stretch'). Upon completion of the rehabilitation programme patients were asked to return to the hospital for repeat physiological and functional assessments.

\subsubsection{Cardiac and respiratory data analysis}

\subsubsection{HRV analysis}

ECG recordings were assessed for quality by human observation using the Spacelabs Pathfinder system (Spacelabs Healthcare Ltd., Hertford, UK), primarily to verify the absence of excessive noise or artefact. Beat- 
to-beat cardiac intervals (RR) were then measured automatically by the Pathfinder system (using a proprietary algorithm) and any spurious data points (artefacts) were removed manually prior to subsequent analysis. The RR data were then analysed using the Kubios HRV software package (version 3.1; [134]) in three separate but contiguous sections covering the Pre-exercise (rest), Exercise and Recovery (post-exercise) stages. The RR data within each of these sections was first detrended using the 'Smooth priors' function (Lambda $=500$ ). Heart rate (HR) and HRV variables were next quantified according to the European Society of Cardiology Task Force guidelines [135]: (1) RMSSD (the square root of the mean squared differences of successive RR intervals), (2) SDRR (the standard deviation of RR intervals), (3) Total Power (the total amount of 'power' (variance) in the bandwidth $\sim 0$ to $0.4 \mathrm{~Hz}$ ), (4) VLF (the very low frequency power, in the bandwidth 0.003-0.1 Hz), (5) LF (the lowfrequency power, in the bandwidth 0.04-0.15 Hz), (6) HF (the high-frequency power, in the bandwidth 0.15-0.4 $\mathrm{Hz}$ ), (7) LFn (the normalised low frequency power): LFn=LF/(Total Power - VLF), (8) HFn (the normalised highfrequency power): HFn=HF/(Total Power - VLF)] and (9) the ratio LF/HF.

\subsubsection{PRSA analysis}

The PRSA procedure [29] was used to quantify the acceleration capacity (AC) and deceleration capacity (DC): (1) multiple DC and AC 'anchor points' were identified, respectively, as those RR intervals longer than or shorter than the preceding RR interval; (2) a truncated RR time series was defined around each of the anchor points (a 61-point series, including 30 points preceding and following the anchor point); (3) the truncated RR time-series were aligned (separately for DC and AC anchor points) and averaged to form the PRSA time-series; (4) DC and AC were calculated as $\left[\left(X_{0}+X_{1}\right)-\left(X_{-1}+X_{-2}\right)\right] / 4$, where $X_{0}$ and $X_{1}$ are the averaged RR intervals at the anchor point and the immediately following point, and $\mathrm{X}_{-1}$ and $\mathrm{X}_{-2}$ are the two averaged RR intervals immediately preceding the anchor point. Group mean Acceleration and Deceleration vectors (used for visualization) were calculated after standardising the individual RR time-series (to minimize inter-individual differences in resting $\mathrm{RR}$ ) - this was performed by subtracting the mean of the 12 RR intervals immediately preceding interval $\mathrm{X}_{-2}$ from each RR value in the time-series.

\subsubsection{Statistical analysis of HRV and PRSA data}

Matlab (version 2017b, The Mathworks, Cambridge, UK) was used to perform descriptive statistical analysis of the HRV and PRSA data, and to evaluate the nature of their distributions. The One-Sample KolomogorovSmirnov Test was used to test the null hypothesis that each group-wide HRV and PRSA-derived variable belonged to a standard normal distribution. If this assumption was violated then the influence of the rehabilitation intervention on each variable was assessed using Wilcoxon Signed Rank tests, and data were 
presented as Median (M) and Lower/Upper Quartile (Q1, Q2) values. One-sample Kolmogorov-Smirnov tests showed that none of the quantified variables were Normally-distributed. Consequently, the Wilcoxon Signedrank test was used to assess the influence of PR on each variable. Statistical significance was assumed at the $\mathrm{p}<0.05$ level.

\subsubsection{Oxygen uptake analysis}

Each participant's $\dot{\mathrm{V}}_{2}$ response to the constant workload exercise test (day 3 protocol) was first plotted to check its structure, and to identify any artefacts in the data (which could arise, for example, owing to swallowing or coughing, or as a result of technical problems such as face-mask loosening). In accord with previous oxygen uptake kinetics studies [16] our intention was to model participants' individual temporal $\mathrm{V}_{2}$ responses using a single exponential model (Equation 1):

$$
\dot{\mathrm{V}} \mathrm{O}_{2}=\mathrm{A} *\left(1-\mathrm{e}^{-(\mathrm{t}-\delta / \mathrm{\tau}}\right)
$$

where " $\mathrm{t}$ " is time, $\mathrm{A}$ is the amplitude of the response (maximum value of $\dot{\mathrm{V}} \mathrm{O}_{2}$, with baseline $\dot{\mathrm{VO}}_{2}$ immediately prior to exercise set to zero), $\delta$ is a time delay and $\tau$ is the time constant. However, we did not observe evidence of so-called Phase I type behaviour (an exponential rise in $\mathrm{VO}_{2}$ attributed to an increase in pulmonary blood flow at the onset of exercise [136] [137]) in any of our data so we chose to remove the time-delay $(\delta=0)$. Neither did we observe Phase III ( $\mathrm{VO}_{2}$ slow component) behaviour, in common with previous work [16]. We therefore chose to perform our analysis solely in terms of the mean response time (MRT), which reflects the time course of the entire $\mathrm{VO}_{2}$ response (Equation (2)):

$$
\dot{\mathrm{VO}} \mathrm{O}_{2}=\mathrm{A} *\left(1-\mathrm{e}^{-\mathrm{t} / \mathrm{MRT}}\right)
$$

The individually normalised $\mathrm{V}_{2}$ responses were modelled using Matlab's Curve Fitting Tool (non-linear least squares method) to determine variables A and MRT and their associated 95\% confidence bounds, as well as the goodness of fit of the model. To facilitate group averaging of the individual responses all data were next interpolated so that $\dot{\mathrm{VO}}_{2}$ was defined uniformly at 0.5 -second intervals. The group-averaged $\mathrm{VO}_{2}$ response was then separately modelled (using equation (2)) for the pre- and post-PR conditions. Finally, the group-averaged responses were re-sampled at 30-second intervals to facilitate pre-PR vs post-PR statistical comparison at 12 discrete time-points (over six minutes) during exercise. 


\subsubsection{Heart rate dynamics analysis}

Participants' heart rate responses to the constant workload exercise test (day 3 protocol) were analysed analogously to the methods described for oxygen uptake analysis in Section 3.2.5.4 (using Equation 3):

$$
H R=A *\left(1-e^{-t / M R T}\right)
$$

\subsubsection{Oxygen uptake efficiency slope}

OUES was calculated from the linear relationship between $\mathrm{VO}_{2}$ and the logarithm of $\mathrm{VE}$, as measured during the incremental exercise tests (day 2 protocol). This relationship can be expressed as:

$$
\dot{\mathrm{V}} \mathrm{O}_{2}=\mathrm{a} * \log _{10} \mathrm{VE}+\mathrm{b}
$$

where OUES is equal to the slope ' $a$ ' of the $\dot{V O}_{2}$ vs $\log { }_{10}$ VE plot $[18,19]$.

\subsubsection{Statistical analysis of oxygen uptake dynamics, heart rate dynamics and OUES data}

Matlab (version 2017b, The Mathworks, Cambridge, UK) was used to model the temporal $\mathrm{VO}_{2}$ and $\mathrm{HR}$ data (Matlab Curve Fitting Toolbox) and to perform descriptive statistical analysis for each variable calculated from the modelling analysis ('A', 'MRT') and from the OUES analysis ('a'). The One-Sample Kolomogorov-Smirnov Test was used to test the null hypothesis that data obeyed Normal distributions. If this assumption was violated, then the influence of the rehabilitation intervention on each variable was assessed using Wilcoxon Signed Rank tests. One-sample Kolmogorov-Smirnov tests showed that none of the quantified variables were Normallydistributed. Consequently, the Wilcoxon Signed-rank test was used to assess the influence of PR for each variable. Statistical significance was assumed at the $p<0.05$ level. 


\section{Chapter 4}

\section{Patients' experiences of coping with Idiopathic Pulmonary Fibrosis and their recommendations for its clinical management}

\subsection{Overview}

This chapter describes the results of an investigation that sought to assess the multiple impacts of being diagnosed, and then living with, IPF. The primary aim of this study was to explore the idiographic (i.e. individual case-specific, as distinct from general) experiences of patients with IPF within our local health board in South West Wales (Abertawe Bro Morgannwg University Health Board), and thus to improve our understanding of the physical, psychological and social impacts of people living with IPF in this region. In so doing we sought to better understand patients' individual stories and to more effectively identify common themes amongst the patient group. The methods used to collect, analyse and interpret the data presented were detailed in Section 3.1.3.

\subsection{Results}

A total of 50 IPF patients registered in the support group were contacted by post and 10 volunteered to take part in the study. No replies were obtained from the other 40 individuals, and no explanations were sought from those who did not respond (in line with our ethical approval). After analysing the transcripts from these ten participants the research team considered that data saturation had been reached and so no additional patients were invited to participate. The study participants ranged in age from 53 to 81 years (mean $=70.5 \pm$ 10.4 years), one of whom was female. Participants reported durations of disease onset ranging from 7 months to 10 years: onset under one year - five patients; onset 3-5 years (two patients); onset 8-10 years - three patients. Only one participant used oxygen during the interview, while two participants reported using oxygen during strenuous activities in daily life. All participants were Caucasian. Eight participants attended the interview on their own (one of whom was interviewed at home) and two attended with their spouses. In these latter interviews, the participant's spouse contributed to the discussion, simultaneously offering verification of the participant's responses, but also often encouraging further reflection or interpretation from the participant. Transcripts of the discussions with each participant are presented in Appendix B. 
Inductive analysis of the patient interviews led to identification of seven second order themes and eleven first order themes that are represented by two General Dimensions: Patient experience with the condition and Patient-led recommendations for practice. An overview of these themes and their hierarchical relationships with each other is shown in Figure 4.1.
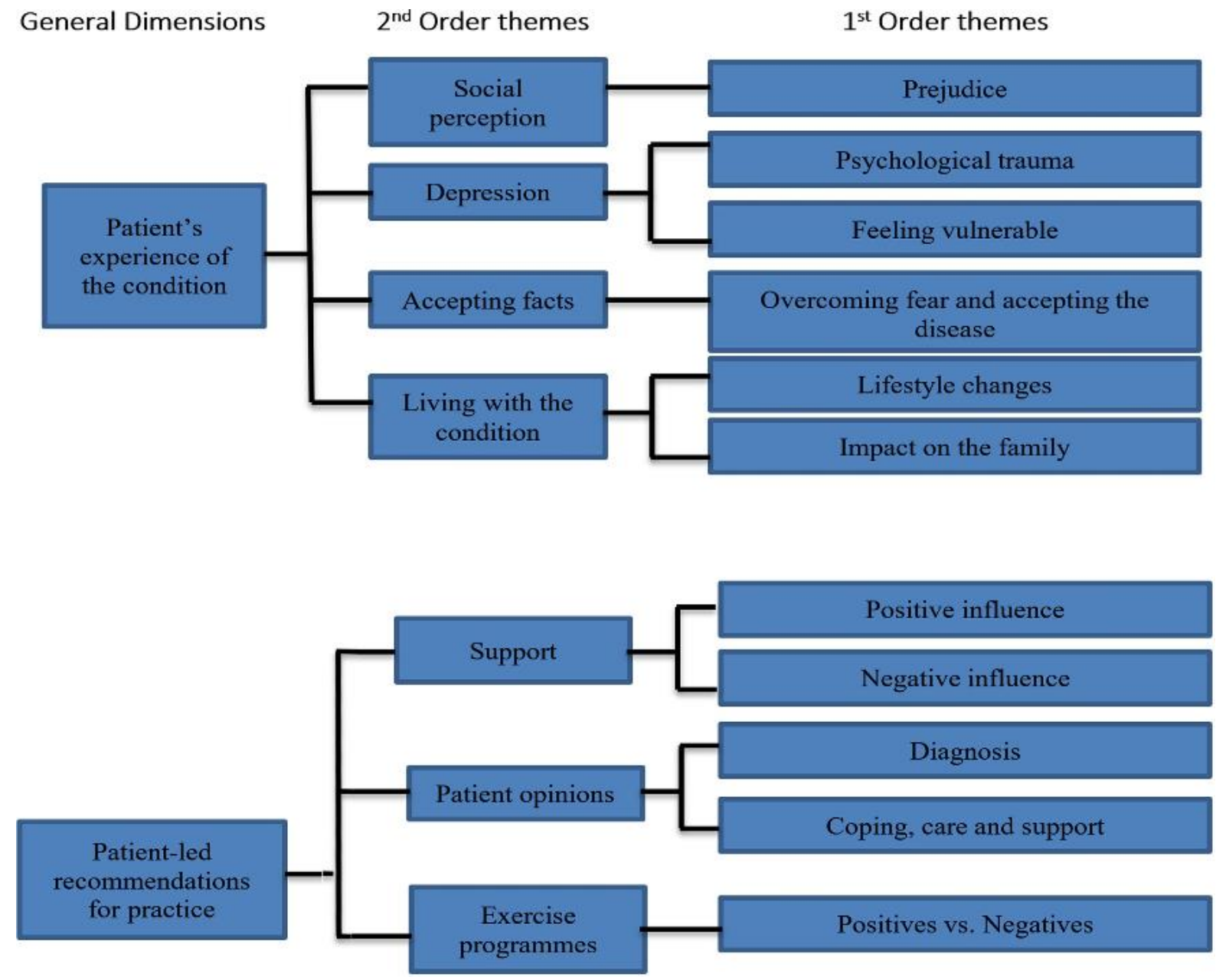

Figure 4.1: Key themes representing patients' experiences of IPF and patient-led recommendations for practice

\subsubsection{General Dimension 1: Patients' experiences of IPF}

This General Dimension was underpinned by 4 second order themes (Social perception, Depression, accepting facts, and Living with the condition) that mainly describe the psychosocial impact of the disease on the patient, how it has affected his or her life, and the impact of IPF on the patient's family members. These second order themes were derived from (and reflect) both negative and positive responses from the participants and were categorised collectively as they relate to the same shared theme. The theme explanations below illustrate the positive and negative responses provided by participants in relation to each theme. 


\subsubsection{Social Perception}

In this context, 'social perception' describes an individual's experiences of negotiating the social implications of IPF, including their feelings about how other people perceive and treat them and how these impacts on their psychological well-being. Across all the interviews, it was evident that participants and their close family members had little or no knowledge of IPF before diagnosis, and most had not even heard the phrase IPF previously. This lack of public awareness could be due to the limited availability of information about the disease: "A lot of people don't know what it is. They understand lung cancer but when it comes to IPF they are like 'what is that?"' (Patient J). This can lead to perceived prejudice: "When people see you coughing, they say 'take cough medicine, take this, take that', they don't understand it" (Patient B) and "they would say, 'why you are panting when you are doing nothing literally?'” (Patient B). When patients face these kinds of responses on a daily basis they feel isolated and somewhat stigmatised, which appeared to be contributing factors to the onset of depressive symptoms in some patients.

\subsubsection{Depression}

Most of the patients interviewed displayed symptoms consistent with depression or low mood. This is likely attributable to the psychological trauma associated with their disease, as noted by one participant who commented on what their diagnosis meant to them: "Death. Certain, definite death." (Patient H). Aside from feelings of depression a sense of vulnerability was also apparent among participants, adding to the feelings of depression, and this appeared to stem from their lack of knowledge about the disease. The comments of two participants illustrate this: "Lack of information always causes more worries" (Patient A) and "Well, I'm in the dark about what you can do to help the condition" (Patient B). Inaccurate or misinterpreted information about the nature and prognostic implications of IPF appears to be a common problem, as reflected in the perceptions: "If I was diagnosed with lung cancer it would have knocked me over, but knowing I have a fighting chance as things are, it's not bothering me" (Patient E), and "I was pleased it wasn't cancer" (Patient $\mathrm{H}$ ).

The psychological trauma caused by the disease also appears to derive from altered perceptions of self: "I feel like a battery; your energy levels go down and you would have to stop" (Patient J). When patients could not live up to their self-expectations and their previous self-perceptions of ability, they experienced feelings of self-pity that further fuelled their anxieties about the debilitating nature of the disease. As one participant commented: "Sometimes I feel ashamed about the things I can't do" (Patient A). Another participant explained: "If I go out I have to plan a route, that I know which is flat. So that I know, if I got to that point I can get back. You have to think 'if I can't come back who am I gonna call to come pick me up?' You know, things like that. It really takes it out of you, it's surprising to see how much it takes out of you. After being there and doing something I feel like 
an old man of 90." (Patient G, age 53). Furthermore, these IPF patients often experienced regret and frustration mainly due to the morbidity of the disease and because they were being prevented from living their later years as planned: "I do get frustrated when it physically affects me, not being able to do things that I planned to do" (Patient J) and "You plan to do all these things that you wanted to do all these years. But here we are." (Patient I).

\subsubsection{Accepting facts}

'Accepting facts' refers to the stage at which all participants indicated they had started to come to terms with the condition and attempted to move on with their lives. As the quotes below illustrate, usually this began with accepting the fact that they have the disease and it ended with the sense of achievement gained from being able to cope with (and adapt to) living with the disease. When accepting the facts about, and the consequences of, the disease, most participants were initially optimistic: "I was determined to live my life to the fullest" (Patient A) and "I told myself 'you have to try anything and everything"' (Patient C).

This positivity and optimism were enhanced further when individuals felt that their lives mattered, thereby nurturing their sense of self-worth. This derived in part from knowing that there are people and groups in society who care about them: "I've had a leaflet from the lung association" (Patient B). Once these participants had achieved acceptance of the disease, they were able to adapt to the disease accordingly: "I learnt a few things like when it comes to climbing steps it's easier to breathe in when you take off the step and breath out when you land on the step" (Patient G) and "If I am going out I need to plan a route which I know is flat" (Patient $\mathrm{G)}$.

Following on from this acceptance and adjustment, some participants attempted to engage in physical activities, the successful completion of which gave them a sense of achievement. This was cause for renewed optimism, and an increased commitment to self-care as they looked to the future with hope: "It was keeping my lungs clear (now I have a lot of mucus and phlegm), and it felt better. When I was done, you felt that you have achieved something" (Patient F). Hearing participants' experiences, it was evident that overcoming fear of the disease and accepting the facts about it represented the initial stage of moving towards an elevated quality of life; individuals had a sense of starting to live again, despite living with the condition, and of achieving some normality to their day-to-day lives. Once they had successfully navigated through this life experience, patients were often able to 'move on', overcoming the psychological trauma caused by the diagnosis and associated morbidity of the disease. 


\subsubsection{Living with the condition}

These themes describe how the disease affected the daily lives of the participants and the adaptations they had made to cope with the condition on a daily basis. Overwhelmingly, IPF had a huge impact on the participants' lifestyles: "I used to play a lot of golf, swimming, cricket, you name it. I've had no problem, but now I'm a television viewer" (Patient B) and "I've sometimes found lifting whatever across the garden these days is difficult, but before it was easy" (Patient E).

Most of the interviewees had to change their lifestyles completely following diagnosis, adjusting to living with the condition through planning and prioritizing activities. They mentioned "finding the simplest way to do things" and "I try to keep everything in precise order, so things at the same place all the time" (Patient A) and "It's sort of reorganizing your life, try and do everything in one place before you move to another" (Patient B). Such modifications helped significantly in overcoming the obstacles they faced due to the debilitating nature of the disease. Participants' comments showed that the disease also affected their family members: "Then my daughter read it and she was badly distressed, and she kept it from me. When it's sitting there in front of you, you think OMG [Oh My God]" Patient $\mathrm{H}$ ) and "My son had a shock when he saw me with the oxygen" (Patient F). Interestingly, the participants did not discuss coping strategies employed by their family members, but some did mention protective behaviour in both directions. Some participants mentioned protecting their family members, and others noted that family members protected them, from some of the stark realities of the disease: "My daughter knows, my husband knows. He has been very protective in a loving way. My son haven't told him" (Patient $\mathrm{H})$.

\subsubsection{General Dimension 2: Patient-led recommendations for practice}

The second general dimension was made by 3 second order themes (support, patient opinions and, exercise programme) which mainly describes patients' opinion and thoughts on how to minimise the impact of IPF on daily life and the positives and negatives of support available for IPF patients. As above the theme explanations illustrates both the positive and negative responses given by the participants in relation to each theme.

\subsubsection{Immediate responses to diagnosis}

Receiving initial information about IPF at the point of diagnosis was a traumatic experience for participants. Most had never heard of the condition previously and the information presented a stark and upsetting 
prognosis: "It came as a little bit of a shock. It affected me" (Patient I) and "Very shocked. Really upset at the time. Coming to terms with it slowly" (Patient H). Two participants commented about the way in which they were told about the disease: "They gave me a booklet and I wish I didn't read it" (Patient $\mathrm{H}$ ) and "To give it to someone as soon as they diagnosed it, I think it was not a good idea" (Patient G). The initial shock and unexpected diagnosis of a disease about which they were unaware left many patients looking for someone to blame, as they felt somehow victimised by their diagnosis. However, they were unable to attribute this blame to someone or something, which only added to the immense frustration and psychological trauma caused by their diagnosis: "I feel picked on, I feel I haven't smoked for all these years, I don't drink, I'm not overly fat, why is this happening to me?" (Patient $\mathrm{G}$ ).

Each of the study participants had an individual perspective on how to cope with the disease and suggestions on how to improve the care and support available for IPF patients. Overwhelmingly, one of the key factors identified was the lack of available and appropriate information on the disease. Both ignorance and ambiguity about the disease contributed to the trauma that they were already experiencing with their diagnosis: "They say you are like this and that's how you are going to end up like. And you think, what's going to happen in between?" (Patient G). Participants offered a number of suggestions on how to address this, including involving patients in developing materials for other sufferers and giving patients a role in educating health professionals, to help them to understand the patient's experience of diagnosis and the disease trajectory. One participant suggested that "Initially I will direct them to our support group. Then I would ask someone who had experience of the condition and the rehabilitation programme to talk about rehab [sic], who can pass on their true feelings - not a clinical thing, somebody talking from the heart who had done it" (Patient D). Another commented "I think you need it in age groups, which would help" (Patient G), and another felt that "You should find out all the facts, face up to the facts, accept what is inevitable and live accordingly" (Patient A). They felt that these strategies would help future IPF patients to appropriately fill the information gap they had experienced when they were diagnosed with the disease.

\subsubsection{Support}

Participants' comments indicated that support from others could be perceived as either a positive or a negative influence. Most of the participants experienced positive support after their diagnosis with IPF, from either family or peers and not surprisingly this was viewed as very important in helping them to accept the disease and to overcome the depression they experienced: "They would do anything if I ask my family" (Patient A). The peer support they experienced mainly came from the monthly support group that these participants attended and, as was the case with family support, support from others with the disease was perceived as vital in 
overcoming feelings of depression and adapting to living with the condition: "The positive is that l've met people similar, some of them are very nice' (Patient A) and "There was company, people with the same disease. I found I was looking forward to going. And I thought it was doing me good" (Patient $\mathrm{H}$ ). Others discussed the informal educational role this peer support played: "We have a cup of tea; cake and we talk about how things are. They get people from different ways of life to talk about the problem" (Patient F) and "Just having a chat about families and how they are coping, you know that's the biggest part of it. Getting together and talking to people with the same problem' (Patient F). Some found comfort in just seeing other people who are in a worse condition than them: "...but it was nice to see there were people worse than I was" (Patient G).

Although the support group was an incredibly powerful coping mechanism for the participants, its influence was not consistently positive: "I couldn't help thinking that this is like Shipman's waiting room", waiting for each one to pop off you know. Because there is always news that somebody is gone and that's a person you know and I think, my name would be there one day wouldn't it?" (Patient A) ["Dr Harold Shipman was a notorious General Practitioner in the UK who was convicted of murdering (via euthanasia) several of his elderly patients]. This patient also discussed the impact of a shared common fate: "...down turn because you all know that you are going sooner than you'd like to be".

Family support was perceived in a similar vein: overall it was received positively but this was not always the case. For example, a number of respondents discussed that family members contributed to their lack of independence by making decisions about their lifestyles for them, or by doing things for them that (with some effort) they were still able to do for themselves. One participant commented "I got told off by my daughter for cutting the grass. But I got to do it. I got to do it when I get the chance" (Patient F), whilst another noted "Eventually she is talking about us moving downstairs and staying downstairs once it gets to a certain point well I don't want that. I'd rather take 10 minutes to go upstairs to go to bed than staying downstairs all the time" (Patient G).

\subsubsection{Exercise programmes}

Study participants felt that physical exercise could benefit them physically and could positively influence their mental well-being, helping them to deal with their feelings of depression and to simply carry on with life. They discussed the benefits they had experienced from exercise: "Mentally I feel good" (Patient D), "I find it useful for circulation on the legs" (Patient I), "I'm sleeping better on the days I was doing it" (Patient G) and "I found after exercising I slept better, woke up better and it improved my flexibility. Mentally you are feeling better too and it helps" (Patient I). However, participants' experiences of exercise did not always remain positive over time 
owing to the high oxygen demand of exercise and the negative effects of this for some patients: "I found it a struggle" (Patient C) and "I found it was a struggle, because I'm out of breath when I do it" (Patient D).

Patients' comments indicated that they preferred group activities when it came to exercise programmes, for example: "It was about meeting others too" (Patient C) and "You met people and after that you got a cup of tea, sat down and had a chat. It was nice. But you won't get that in a gym" (Patient G) and "There was company, people with the same disease. I found I was looking forward to going. And I thought it was doing me good" (Patient $\mathrm{H}$ ).

\subsection{Discussion}

IPF is a life-limiting disease that has no curative options [36] and this condition is little-recognised and poorlyunderstood by the general public. From a patient's perspective a diagnosis of IPF is therefore often completely unexpected and confusing, provoking a range of psychological responses that cause much anxiety. A better appreciation of patients' personal experiences of being diagnosed (and then coping) with IPF is critical to changing the way we educate and support newly-diagnosed patients about their condition. Our exploration of individual experiences of IPF offers insight into the psychosocial implications of IPF, its impacts on psychological and physical well-being, and the physical and psychological coping strategies that patients adopt to cope with these. This has enabled us to present here some patient-led recommendations for healthcare workers to support IPF patients from the point of diagnosis and throughout the progression of their disease. We analysed our data inductively to allow themes to be generated from the data rather than using a deductive theory-driven approach [110].

\subsubsection{Patients' experiences of IPF}

\subsubsection{IPF and need-thwarting}

Our participants told us that they had experienced insensitive and unsympathetic comments regarding their condition and the struggles they faced each day, leading them to feel less related to (and more isolated from) others in wider society (reduced relatedness satisfaction). The majority of participants said that, because of this, they had limited their social interactions and confined themselves to a 'safe space' among family, close friends and sometimes the monthly IPF support group. Russel et al. [138] claimed that a poor understanding of IPF amongst the general public contributes to the emotional impact of IPF on patients (depression, fear, frustration and isolation), causing them to withdraw from social relationships. This reduced social interaction results in an unfulfilled need for relatedness (need-thwarting) in these individuals and negatively influences their overall well-being and quality of life. Schoenheit et al. [90] made similar observations, claiming that IPF 
patients express difficulties in maintaining relationships owing to others' lack of awareness and understanding of the disease. Sampson et al. [139] observed that deterioration in health caused patients to restrict their activities (by confining them to indoors) rather than seeking assistance to maximise their functional activities (through socialising and conducting outdoor activities), thereby increasing the risk of social isolation and leading to diminished possibilities. Similarly Swigris et al. [140] reported that some patients avoided engaging with crowds of people simply because of the fear of catching a respiratory disease that could lead to their demise. Bonella et al. [141] suggested that awareness of IPF among the general public should be increased through campaigns emphasising the chronic and debilitating nature of the disease. Our participants' responses were consistent with depressed mood, indicating that their sense of incompetence, lack of autonomy and lack of intrinsic motivation were having a substantial negative impact on their well-being.

Not surprisingly, given its physically-restrictive nature, IPF had a substantial impact on patients' satisfaction regarding the need to feel competent. This impact was especially apparent in the execution of what were previously simple, taken-for-granted daily activities. The patients we interviewed often experienced signs of breathlessness and fatigue during the simplest of physical activities like walking or moving an object from one place to another. In addition, most of our participants had previously been involved in sport or other recreational activities such as walking prior to the onset of IPF, and no longer being able to participate in these activities further thwarted their need to feel competent. This need-thwarting leads to frustration and was one of the most common observations we made during our interviews, along with a sense of shame and self-pity that stemmed from the morbidity impact of IPF. Patients' reduced competence satisfaction was fuelled by the thwarting of their need for autonomy as most had to seek help in carrying out the activities of daily living. Often family members stepped in to help these patients even before their help was solicited; whilst participants appreciated their help, family members were unknowingly undermining the individual's sense of autonomy and thus their competence satisfaction. Previous studies have similarly reported that patients' inability to perform routine daily tasks negatively impacts on their emotional wellbeing [90] [140]. Patients interviewed by Swigris et al. [140] commented that the most detrimental aspect of this disease is the fact that they are less independent than previously, leading to a loss of privacy because they needed help from others.

A lack of understanding about the disease compounds this situation. Patients are reluctant to participate in physical activity because they are afraid of its possible impact on their condition, further reducing autonomy and competence-need satisfaction. Carers are often unsure how to help patients and express concerns regarding the monitoring of breathing, coughing and the use of oxygen [139]. Sampson et al. [139] reported that patient-clinician consultations are mostly disconnected from the demands of practical day-to-day life. 
Patients often feel that they cannot interpret disease-focused assessments (like lung function tests) in relation to predicted future exercise capacity, causing them to simply avoid physical activity (presumably as a conservative approach to avoiding exacerbation). Cicutto et al. [142] also found similar attitudes to exercise in COPD patients, as did Fernandez et al. [143] in cancer patients, illustrating that this is a common observation in the literature relating to many chronic diseases.

\subsubsection{Coping mechanisms and re-establishing need-satisfaction}

There was evidence that our participants had also developed coping mechanisms that employed emotion-, problem-, appraisal-, approach- or support-focused strategies that helped them restore need-satisfaction after diagnosis. It was apparent that for many individuals the key to coping with IPF was an acceptance of the reality of their condition, as this subsequently led to a sense of optimism.

During the early stages of diagnosis (following initial emotional upheaval) participants used appraisal-focused coping strategies to purposely change their perspectives - this allowed them to start thinking more positively and to gain some determination to live the remainder of life to the fullest. One such example of this positive thinking and optimism was seen in a patient who had bought football tickets for the following season whilst knowing that his condition meant he had a relatively low likelihood of living to watch the games. Others have also observed that patients and family members try to maintain a normal life for as long as possible by living in the moment, while acknowledging that this is difficult as the disease progresses [144].

Participants used emotion-focused coping to overcome depression and to face the reality of their condition, mainly through the opportunities for emotional expression that the IPF support group offered. As Folkman and Lazarus [113] suggested, emotion-focused coping was employed alongside social support from others who shared their experience in the support group. The simultaneous use of emotion-focused coping and social support was also evident in the participants' discussions of how family and friends had helped them to overcome the initial trauma and fear of diagnosis. Importantly, their recognition of the support and care from others helped to restore both lost self-worth and relatedness-satisfaction. The support group also functioned to facilitate problem-focused coping as individuals could exchange knowledge and experiences about the disease and provide tips on how to cope with the condition, which they would be unlikely to receive from a medical professional. There are however some negative associations with support groups - for example, Sampson et al. [139] noted that seeing people who are further ahead in terms of disease progression threatens the ability of some patients to cope with the condition. 
Participants' effective employment of support-, appraisal- and emotion-focused strategies for coping with the wider psychological and personal implications of IPF meant that they were then able to use problem-focused coping to deal with daily challenges. They did so by adapting to their condition using small adjustments to the ways in which they conducted previously taken-for-granted activities. Sampson et al. [139] stated that patients use a day-by-day coping strategy to adapt to their condition. Swigris et al. [140] observed that the majority of patients in their study were forced to plan everything ahead, leading them to analyze every activity before starting it. Being able to accommodate the challenges IPF presents to once again conduct activities of daily living, such as going for a walk, provided a sense of achievement and led to a regained sense of competence and enhanced well-being. Some of our patients found comfort in a somewhat unorthodox manner by feeling good about themselves after seeing patients who were in a much worse condition than themselves.

\subsubsection{Patient-led recommendations for need-satisfaction}

\subsubsection{Information}

Lack of information is one of the main issues that IPF patients face. This led many participants to feel uncertain about what they could and could not do after diagnosis. They felt uninformed and unable to make accurate judgements of their competence to carry out physical activities, resulting in the adoption of a sedentary lifestyle. The information given to these patients on IPF at the point of diagnosis was presented in an information booklet including general information on the disease, which emphasised life expectancy and what the patient could expect to happen towards the end of their life. However, it offered little information on what patients might expect to experience in the shorter term or how they might best cope with the condition. This often-caused shock, anxiety and trauma, and subsequently led to depression-like symptoms in these patients. It should be noted that our patents were perhaps advantaged in receiving such information: patients have previously commented that they do not receive sufficient information on the practical management of the disease (ranging from advice on oxygen therapy, nutrition, exercise, the management of cough, managing breathlessness and, most importantly, how to cope with the disease towards the end of life) $[90,138,139$, 141]. Others have noted that learning about the fatality of the disease is overwhelming for patients, and that they often find the amount of information they received overpowering; these patients instead prefer to be given information about the disease gradually [144]. In fact Bonella et al. [141] highlighted 'improved information sources' as one of the five unmet needs in IPF care after consulting eleven European patient advocacy groups (an initiative that led to the development of the European IPF Patients' Charter). Based on our participants' stories we recommend that the information given by health professionals should focus on living with IPF, maintaining quality of life through lifestyle changes (planning and prioritising) and encouraging patients to remain as physically active as possible. This would help patients to maintain feelings 
of competence and autonomy and would encourage a proactive coping approach in patients from the point of diagnosis.

\subsubsection{Support for need satisfaction}

Family support plays a key role in maintaining the well-being of IPF patients, so it would be beneficial if immediate family members also received education about IPF and its effects on the lives of both the patient and themselves. Russel et al. [138] found that caregivers were inadequately prepared for the role of the caregiver owing to a lack of information about the disease and the absence of psychological support, resulting in an inability to relate to the patient. Sampson et al. [139] recommended that the role of the carer should change from 'passive observer' to 'active member' throughout the patient's healthcare pathway, and that they should have access to better advice and support to fulfil their role in managing the domestic environment for patients. This is an important aspect of providing appropriate social support for patients, especially in relation to being physically active. We suggest that an emphasis should be placed on how not to support these patients; for example, immediate intervention to help patients when performing physical activity or activities of daily living could thwart autonomy and competence satisfaction and lead to feelings of shame and subsequently depression. Instead, family members could be encouraged to help patients employ problem-based coping strategies, making lifestyle changes to enable continuation of activities that they performed prior to diagnosis. Furthermore, it is highly recommended that patients join an IPF-specific support group if such a facility is available. This will help them fulfil their needs for relatedness, while allowing them to obtain valuable knowledge about their condition from fellow patients who are experiencing different stages of the disease. Importantly, this will help increase patients' awareness of what to expect in the future, and peer advice will assist them in employing proactive-coping strategies, with a view to maintaining competence and autonomy need satisfaction. Comments from participants in this study indicate that in general patients would benefit from participation in physical activities, especially in a group setting. Such benefits might include improvements in physical or psychological well-being (perhaps helping to restore competence satisfaction or to reduce feelings of social isolation), although further studies are needed to provide evidence of this

\subsection{Strengths of the study}

Our exploration of individual experiences of IPF aims to offer insight into the psychosocial implications of IPF, its impacts on psychological and physical well-being, and the physical and psychological coping strategies that patients adopt to cope with these problems. 
As the psychological implications of an IPF diagnosis is an under-explored area, we analysed our data inductively to allow themes to be generated from the data rather than using a deductive theory-driven approach [110]. Based on our emergent higher-order themes it is apparent that two key theories present particularly useful frameworks with which to interpret our results. These theories are SDT[111] [117] and 2) transactional theory of stress and coping [118].

A brief explanation of the transactional model of stress and coping illustrate its complementarity with SDT, thereby supporting our use here of both theories to improve our insight into patients' personal experiences of IPF. Additional coping mechanisms that we looked for in this study were avoidance-coping (part of emotionfocused coping) and self-regulation and proactive-coping [118]. Avoidance coping is defined as an effort to avoid facing the stressor and could in itself lead to depressive symptoms [119]. Self-regulation and proactivecoping involve determination of potential future stressors and taking action in advance to negate the impact of these [120]. The transactional theory has been used across a range of contexts, for example to understand coping strategies following disaster [121] and the stress and burnout experienced by French elementary school teachers [122].

\subsection{Limitations of the study}

Of the 50 patients approached 40 did not respond and the nature of our recruitment process meant that we had no follow-up information about the reasons for lack of replies. Although our sample was therefore restricted to ten participants, we believe that data saturation had been reached at this level, so we are confident that our results are robust. The demographic of the participants was quite focussed, and this might bias our results to a degree: 1) Participants were all resident in Swansea (South West Wales) or its immediate surrounding areas; 2) Participants were recruited from a single IPF support group which was part of the palliative care pathway of the local health board. This type of support system might not be generally available across other health boards in the UK or in other countries, so our findings (especially those relating to opinions on the support group) will be biased to this demographic; 3 ) The stage of disease progression and the time since diagnosis for our patients ranged from 1-10 years and, although this range is wide, the relatively small number of participants means there was some clustering into early/late stages of disease progression. Recentlydiagnosed participants are likely to have different opinions about the disease and its impact on their lives compared to those who have been living with the disease for much longer. For each of these reasons we must be guarded in our interpretation of the results and in any attempt to generalise the findings to wider populations. 


\subsection{Concluding comments}

This chapter presented the results of a study that evaluated the health and wellbeing experiences of people living with IPF in ABMU Health Board. It offers novel findings that will help to inform much-needed changes in the practice of supporting IPF patients to cope with their diagnosis and disease progression. By interpreting these experiences within the theoretical frameworks offered by self-determination theory and the transactional model of stress and coping, the study has added insight into the mechanisms that underpin the impact of IPF on psychological wellbeing. Furthermore, the recommendations presented are strengthened by their grounding in both theory and practice, and by ensuring that we considered the varying personal experiences that are characteristic of living with IPF. Based on these findings, it is strongly suggested that future support services for patients with IPF would benefit from a clear focus on providing effective and considerate communication about the disease. In addition, there should be a focus on ensuring that patients and their families are better informed about the potential benefits of attending peer support groups, and about the types of coping strategies employed by their peers to 'live better' with their condition. 


\section{Chapter 5}

\section{Heart rate and respiratory responses to incremental (slowly increasing, wide- ranging) metabolic challenge}

\subsection{Overview}

It has been shown previously that IPF patients' peak $\mathrm{V}_{2}$ values were half those of similarly aged healthy individuals, whilst their peak heart rates were $20 \%$ lower and their kinetic HR responses to heavy intensity exercise were 2.5 times slower [16]. These results provide evidence of reduced tissue oxygen utilization following the onset of exercise in people with UIP, which in large part results from impeded central (cardiovascular) oxygen delivery. It is postulated here that an exercise-based physical rehabilitation programme could reasonably be expected to influence cardiovascular function and thus improve systemic oxygen delivery (and peripheral muscle oxygen availability) in these patients.

Heart rate dynamics are thought to provide a good proxy for the response of cardiac output and muscle blood flow to increased metabolic demand [26] [27]. HR responsiveness can be estimated using HRV analysis (HRV; the variation in beat-to-beat duration of the cardiac cycle) [28], which reflects autonomic nervous system (ANS) influences on heart rate. PRSA [29] yields averaged RR time series that separately characterize heart rate modulations that result in either accelerations and decelerations. HRV and PRSA each have their own advantages: HRV yields band-limited estimates of cardiac neural control, allowing separate analysis of sympathetic and parasympathetic modulation; Unlike HRV, PRSA does not require steady-state conditions so it can reflect the dynamic range of cardiac modulations across multiple and transient physiological states. Individuals with COPD display altered HRV during rest and exercise [30] but with a normal diurnal variation [31], and they have a tendency towards reduced PRSA-derived cardiac dynamic capacities that are ameliorated with oxygen therapy [105]. However, neither HRV nor cardiac acceleration/deceleration capacities have been evaluated in individuals with UIP.

This chapter reports the findings of investigations into the influence of an eight-week, exercise-based PR programme on measures of cardiac autonomic control in patients with pulmonary fibrosis (either IPF or UIP). Specifically, validated measures of HRV and of cardiac acceleration and deceleration capacities are compared during periods of rest and exercise, pre- and post- rehabilitation. The methods of physiological measurement and data analysis used were reported in Section 3.2. 


\subsection{Participant characteristics}

Eighteen individuals (13 males, 5 females) agreed to participate in the study but three males later withdrew from the rehabilitation programme for personal reasons. The patients' demographics (age, gender, body mass index) and diagnostic spirometry measures (forced expiratory volume in one second and forced vital capacity) are shown in Table 5.2. Patients continued with their usual medication regimens whilst participating in the study (Table 5.1).

Table 5.1 Participants' drug regimens during the rehabilitation programme

\begin{tabular}{|c|c|c|c|c|}
\hline \multirow{2}{*}{$\begin{array}{l}\text { PATIENT } \\
\text { ID }\end{array}$} & \multicolumn{4}{|c|}{ PRESCRIBED MEDICATION } \\
\hline & Respiratory & Cardiovascular & Diabetic & Other \\
\hline P01 & Nintedanib & $\begin{array}{l}\text { Ramipril } \\
\text { Warfarin }\end{array}$ & - & Tamsulosin \\
\hline P02 & Nintedanib & - & - & Levothyroxine \\
\hline P03 & Nintedanib & - & - & - \\
\hline P04 & - & $\begin{array}{l}\text { Ramipril } \\
\text { Felodipine }\end{array}$ & Metformin & - \\
\hline P05 & - & $\begin{array}{c}\text { Bisoprolol } \\
\text { Candesartan }\end{array}$ & - & - \\
\hline P06 & - & - & - & $\begin{array}{c}\text { Escitalopram } \\
\text { Prednisolone } \\
\text { Hydroxychloroquine } \\
\text { Mycophenolate mofetil }\end{array}$ \\
\hline P07 & $\begin{array}{l}\text { Carbocisteine } \\
\text { Pirfenidone }\end{array}$ & - & - & - \\
\hline P08 & - & Atorvastatin & - & $\begin{array}{l}\text { Bendrofluazide } \\
\text { Pandesartan } \\
\text { Omeprazole }\end{array}$ \\
\hline P09 & Nintedanib & - & - & \\
\hline P10 & Carbocisteine & - & - & Paracetamol \\
\hline P11 & - & - & Metformin & $\begin{array}{l}\text { Hydroxychloroquine } \\
\text { Mycophenolate mofetil } \\
\text { Mirtazapine }\end{array}$ \\
\hline P12 & Pirfenidone & Simvastatin & Metformin & \\
\hline P13 & Pirfenidone & $\begin{array}{l}\text { Bisoprolol } \\
\text { Amlodipine }\end{array}$ & - & $\begin{array}{c}\text { Citalopram } \\
\text { Lansoprazole } \\
\text { Uvistat }\end{array}$ \\
\hline P14 & Nintedanib & Simvastatin & $\begin{array}{l}\text { Empagliflozin } \\
\text { Linagliptin } \\
\text { Metformin }\end{array}$ & Tramadol \\
\hline P15 & - & - & Pioglitazone & $\begin{array}{l}\text { Omeprazole } \\
\text { Fluoxetine }\end{array}$ \\
\hline
\end{tabular}


Table 5.2 Patient demographics. (BMI = body mass index, FEV1 = forced expiratory volume in one second [age-predicted \%], FVC = forced expiratory volume [age-predicted \%]; NA = data not available.)

\begin{tabular}{|c|c|c|c|c|c|c|c|c|}
\hline Patient & Age & Gender & $B M I$ & FEV1 & FVC & FEV1/ & $D L_{C O}$ & $K_{C O}$ \\
\hline$I D$ & (years) & $(M / F)$ & $\left(k g \cdot m^{-2}\right)$ & (litre [\%]) & (litre [\%]) & FVC & \multicolumn{2}{|c|}{ (\% predicted) } \\
\hline P01 & 72 & $M$ & 36.7 & 1.92 [67] & $2.33[62]$ & 0.82 & 50 & 95 \\
\hline P02 & 73 & $\mathrm{~F}$ & 47.7 & $1.46[82]$ & 1.66 [77] & 0.87 & 35 & 70 \\
\hline P03 & 74 & $M$ & 26.3 & $2.34[78]$ & $2.78[63]$ & 0.84 & 40 & 77 \\
\hline P04 & 67 & $\mathrm{~F}$ & 34 & 1.48 [75] & $1.81[76]$ & 0.81 & 43 & 87 \\
\hline P05 & 78 & M & 32.7 & $2.93[130]$ & 3.95 [131] & 0.74 & 46 & 93 \\
\hline P06 & 60 & $\mathrm{~F}$ & 32 & $2.73[88]$ & 3.49 [89] & 0.78 & 55 & 62 \\
\hline P07 & 68 & $M$ & 32.3 & 2.53 [79] & 3.22 [74] & 0.78 & 57 & 75 \\
\hline P08 & 83 & $M$ & 28.7 & 2.21 [85] & $2.90[82]$ & 0.76 & 42 & 78 \\
\hline P09 & 73 & $M$ & 23.1 & $1.93[63]$ & $3.13[52]$ & 0.62 & 52 & 67 \\
\hline P10 & 75 & M & 20.8 & $2.22[93]$ & 3.37 [107] & 0.65 & NA & NA \\
\hline P11 & 68 & $F$ & 30.4 & $1.77[60]$ & $2.12[53]$ & 0.83 & NA & NA \\
\hline P12 & 72 & M & 25.3 & 2.00 [63] & 1.46 [61] & 0.75 & NA & NA \\
\hline P13 & 64 & $\mathrm{~F}$ & 30.1 & $1.12[55]$ & 1.46 [59] & 0.76 & NA & NA \\
\hline P14 & 65 & $M$ & 24.5 & $2.50[78]$ & 3.25 [78] & 0.76 & NA & NA \\
\hline P15 & 55 & $M$ & 29.8 & NA & NA & NA & NA & NA \\
\hline Mean & 69.8 & & 30.2 & 2.0 & 2.6 & 0.76 & 46.6 & 78.2 \\
\hline$S D$ & 7.1 & & 6.4 & 0.5 & 0.8 & 0.06 & 7.3 & 11.4 \\
\hline Range & $55-78$ & & $20.8-47.7$ & $1.12-2.93$ & $1.46-3.95$ & $\begin{array}{c}0.62- \\
0.87\end{array}$ & $\begin{array}{l}35- \\
57\end{array}$ & $67-95$ \\
\hline
\end{tabular}




\subsection{Results of physiological assessment}

It was decided that the most appropriate approach to analysing the physiological data would be to aggregate the results for the entire group (rather than performing sub-group analyses, for example according to age, gender, disease stage or medication regime). This decision was taken mainly because of the relatively small sample size, which would not adequately support a robust statistical analysis of sub-group effects.

Table 5.3 summarises participants' physiological responses to maximal incremental exercise tests, pre- and post-rehabilitation. Individual data are presented in Appendix C (Tables C.3 and C.4). VE, peak $\dot{V}_{2}$, time to peak work rate and OUES were all unchanged following PR, but the peak heart rates and peak work rates were both significantly reduced following PR. Example plots of $\mathrm{V}_{2}$ vs. time (maximal effort) and of the OUES calculation procedure are provided in Appendix C (Figures C.1 and C.2).

Table 5.3: Physiological responses to incremental exercise tests pre- and post-rehabilitation.

\begin{tabular}{|c|c|c|c|}
\hline & Pre-PR: & Post-PR: & $p$ \\
\hline & Mean (SD); Range & Mean (SD); Range & \\
\hline$V E\left(m L \cdot m^{-1} \cdot \mathrm{kg}^{-1}\right)$ & 56.0 (20.78); 22.4-92.3 & $58.1(18.0), 30.7-90.5$ & 0.53 \\
\hline Peak $\dot{V}_{2}\left(L \cdot \mathrm{min}^{-1}\right)$ & $1.30(0.28) ; 0.86-1.81$ & $1.28(0.30) ; 0.83-1.91$ & 0.96 \\
\hline Peak $\dot{V} \mathrm{O}_{2}\left(\mathrm{~mL} \cdot \mathrm{min}^{-1} \cdot \mathrm{kg}^{-1}\right)$ & $15.7(4.4) ; 10.0-28.4$ & $15.2(4.4) ; 8.4-26.2$ & 0.93 \\
\hline Peak HR (bpm) & $120(24) ; 73-159$ & $91(28) ; 67-136$ & 0.0002 \\
\hline Peak Work Rate (Watts) & 105 (35); 29-158 & $78(25) ; 35-128$ & 0.0005 \\
\hline Time to Peak Work Rate (min.) & $11.8(2.2) ; 7.3-16.4$ & $11.4(3.0), 6.1-16.4$ & 0.73 \\
\hline OUES $\left(\mathrm{m} / \cdot \mathrm{min}^{-1} \cdot \mathrm{L}^{-1}\right)$ & 1569 (206); 1275-1943 & 1509 (250); 1121-1929 & 0.14 \\
\hline
\end{tabular}




\subsubsection{Heart Rate Variability}

The HRV indices calculated pre- and post-intervention for the pre-exercise (Rest), Exercise (Ex) and Recover (Rec) periods are shown in Table 5.4 and Figure 5.1. The intervention did not influence any of the HRV variables during the Pre-Exercise $(p=0.14-0.99)$ or Exercise $(p=0.12-0.76)$ states. Heart Rate was greater (and RR was lower) post-intervention during the Recovery state $(p=0.036 ; z=-2.10 ; E S=0.38)$ and both HFn ( $p=0.069 ; z=-1.82$; $E S=0.33)$ and LF/HF ( $p=0.047 ; z=1.99 ; E S=0.36)$ showed trends towards greater high-frequency dominance during Recovery post-intervention (other variables: $p=0.21-0.72$ ). The relative changes in HRV (HR, SDNN, RMSSD, LFn and TP) following the PR intervention are shown in Figure 5.2 (the remaining variables are largely redundant for this purpose as they are closely related to these five variables). 
Table 5.4: HRV indices pre and post PR. Median (M) and the lower (Q1) and upper (Q3) quartile values are shown, separately for the pre-exercise (Rest), Exercise and Recovery states

\begin{tabular}{|c|c|c|c|c|c|c|c|c|c|c|c|c|c|}
\hline & & & $\begin{array}{c}\text { HR } \\
\text { (BPM) }\end{array}$ & $\begin{array}{c}\text { RR } \\
\text { (ms) }\end{array}$ & $\begin{array}{l}\text { SDNN } \\
\text { (ms) }\end{array}$ & $\begin{array}{c}\text { RMSSD } \\
\text { (ms) }\end{array}$ & $\begin{array}{c}\text { VLF } \\
\left(m s^{2}\right)\end{array}$ & $\begin{array}{c}\mathrm{LF} \\
\left(m s^{2}\right)\end{array}$ & $\begin{array}{c}\mathrm{HF} \\
\left(m s^{2}\right)\end{array}$ & $\begin{array}{l}\text { LFN } \\
(\%)\end{array}$ & $\begin{array}{l}\text { HFN } \\
(\%)\end{array}$ & LF/HF & $\begin{array}{c}\mathrm{TP} \\
\left(\mathrm{mS}^{2}\right)\end{array}$ \\
\hline \multirow{10}{*}{$\begin{array}{l}\frac{\propto}{\alpha} \\
\frac{1}{\alpha} \\
\frac{\alpha}{\alpha}\end{array}$} & \multirow{3}{*}{$\begin{array}{l}\ddot{y} \\
\tilde{x}\end{array}$} & $M$ & 85.4 & 702.9 & 14.7 & 13.6 & 6.7 & 57.1 & 19.7 & 56.7 & 43.0 & 1.3 & 94.4 \\
\hline & & Q1 & 74.3 & 586.0 & 8.4 & 11.8 & 3.1 & 10.6 & 11.9 & 41.4 & 30.4 & 0.7 & 35.2 \\
\hline & & Q3 & 102.5 & 816.0 & 27.7 & 18.3 & 37.8 & 162.6 & 72.9 & 67.1 & 57.3 & 2.3 & 249.3 \\
\hline & \multirow{3}{*}{$\stackrel{x}{4}$} & $M$ & 101.0 & 594.2 & 13.5 & 19.9 & 2.9 & 20.0 & 20.0 & 39.6 & 59.4 & 0.7 & 63.0 \\
\hline & & Q1 & 84.2 & 536.4 & 9.4 & 13.3 & 1.2 & 6.6 & 7.6 & 27.5 & 39.4 & 0.4 & 22.0 \\
\hline & & Q3 & 111.9 & 712.0 & 32.9 & 40.9 & 20.9 & 191.4 & 117.8 & 60.1 & 72.0 & 1.5 & 347.2 \\
\hline & \multirow{3}{*}{$\underset{\mathscr{d}}{\stackrel{\bigcup}{\sim}}$} & $M$ & 94.0 & 638.1 & 16.0 & 15.6 & 18.7 & 60.9 & 48.6 & 47.5 & 52.3 & 0.9 & 136.0 \\
\hline & & Q1 & 86.5 & 548.0 & 11.2 & 11.1 & 5.9 & 34.1 & 10.5 & 33.1 & 15.8 & 0.5 & 50.3 \\
\hline & & Q3 & 109.6 & 693.3 & 34.6 & 50.8 & 41.0 & 322.6 & 222.9 & 84.0 & 66.4 & 5.6 & 606.3 \\
\hline & \multirow{3}{*}{$\begin{array}{l}\ddot{\tilde{d}} \\
\stackrel{\simeq}{c}\end{array}$} & $M$ & 86.6 & 692.7 & 15.0 & 23.1 & 9.3 & 72.4 & 53.2 & 61.6 & 38.2 & 1.6 & 153.9 \\
\hline \multirow{8}{*}{$\frac{\frac{r}{a}}{\frac{1}{n}} \frac{0}{0}$} & & Q1 & 79.4 & 641.2 & 12.3 & 15.1 & 4.2 & 42.2 & 15.3 & 39.5 & 19.6 & 0.6 & 93.1 \\
\hline & & Q3 & 93.6 & 755.3 & 20.6 & 31.9 & 42.2 & 147.9 & 123.4 & 80.2 & 59.6 & 4.1 & 210.4 \\
\hline & \multirow{3}{*}{ x } & $M$ & 112.5 & 549.4 & 23.6 & 34.9 & 7.1 & 45.1 & 73.8 & 36.3 & 62.3 & 0.5 & 119.6 \\
\hline & & Q1 & 93.2 & 522.3 & 11.0 & 19.7 & 2.6 & 20.5 & 8.7 & 26.0 & 43.6 & 0.4 & 39.9 \\
\hline & & Q3 & 115.4 & 638.8 & 31.3 & 43.6 & 13.9 & 110.4 & 316.8 & 56.3 & 73.4 & 1.3 & 527.1 \\
\hline & \multirow{3}{*}{$\underset{\mathscr{d}}{\stackrel{d}{u}}$} & $M$ & 110.9 & 541.2 & 16.5 & 26.2 & 5.3 & 60.5 & 43.2 & 34.4 & 64.9 & 0.5 & 143.1 \\
\hline & & Q1 & 90.6 & 513.3 & 12.5 & 17.8 & 2.2 & 15.5 & 11.7 & 30.0 & 32.7 & 0.4 & 46.1 \\
\hline & & Q3 & 116.9 & 665.9 & 30.0 & 43.3 & 12.2 & 146.4 & 173.2 & 67.1 & 69.2 & 2.2 & 349.0 \\
\hline
\end{tabular}


Figure 5.1 HRV indices pre and post PR. Median (M) values are shown, separately for the pre-exercise (Rest), Exercise and Recovery states.
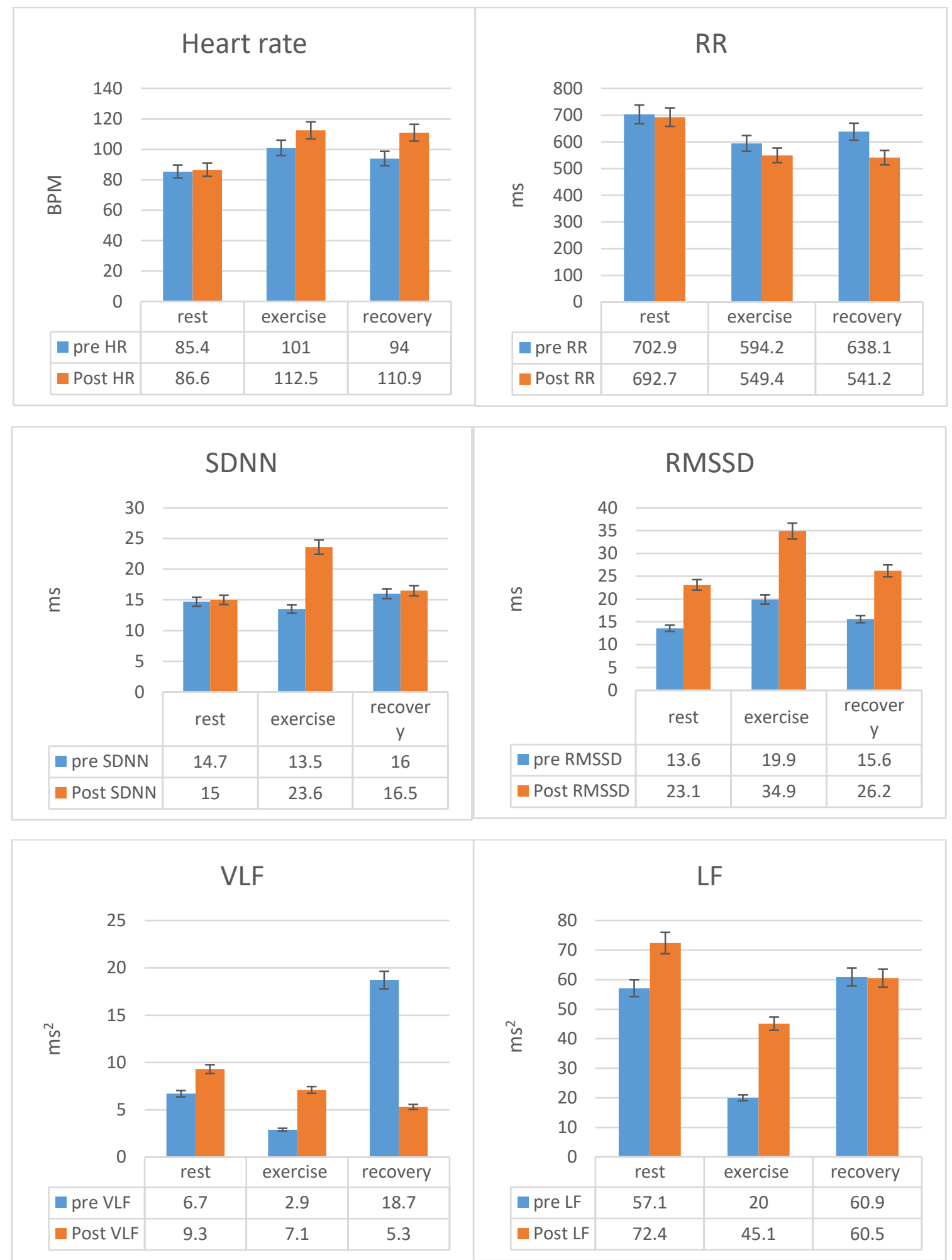

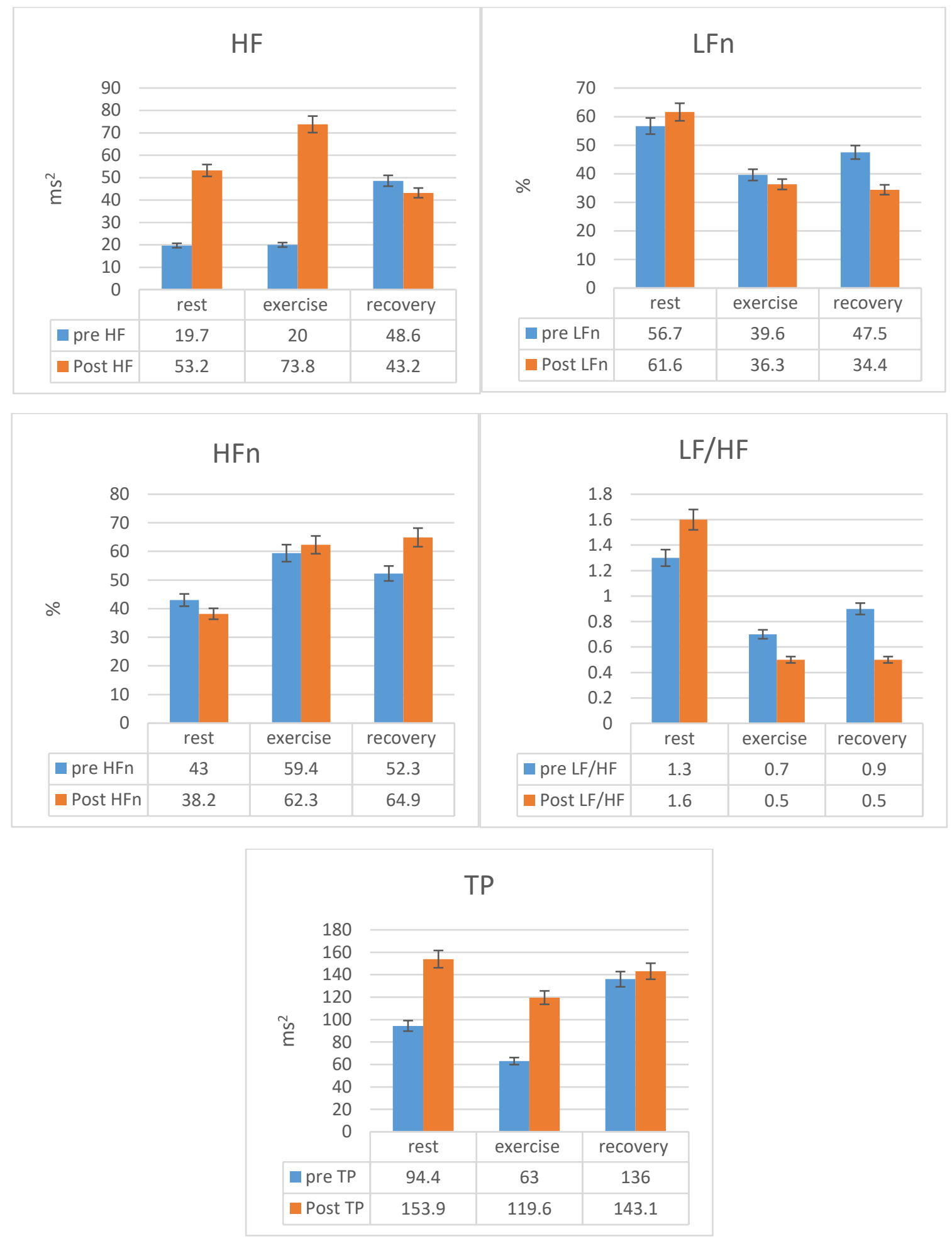


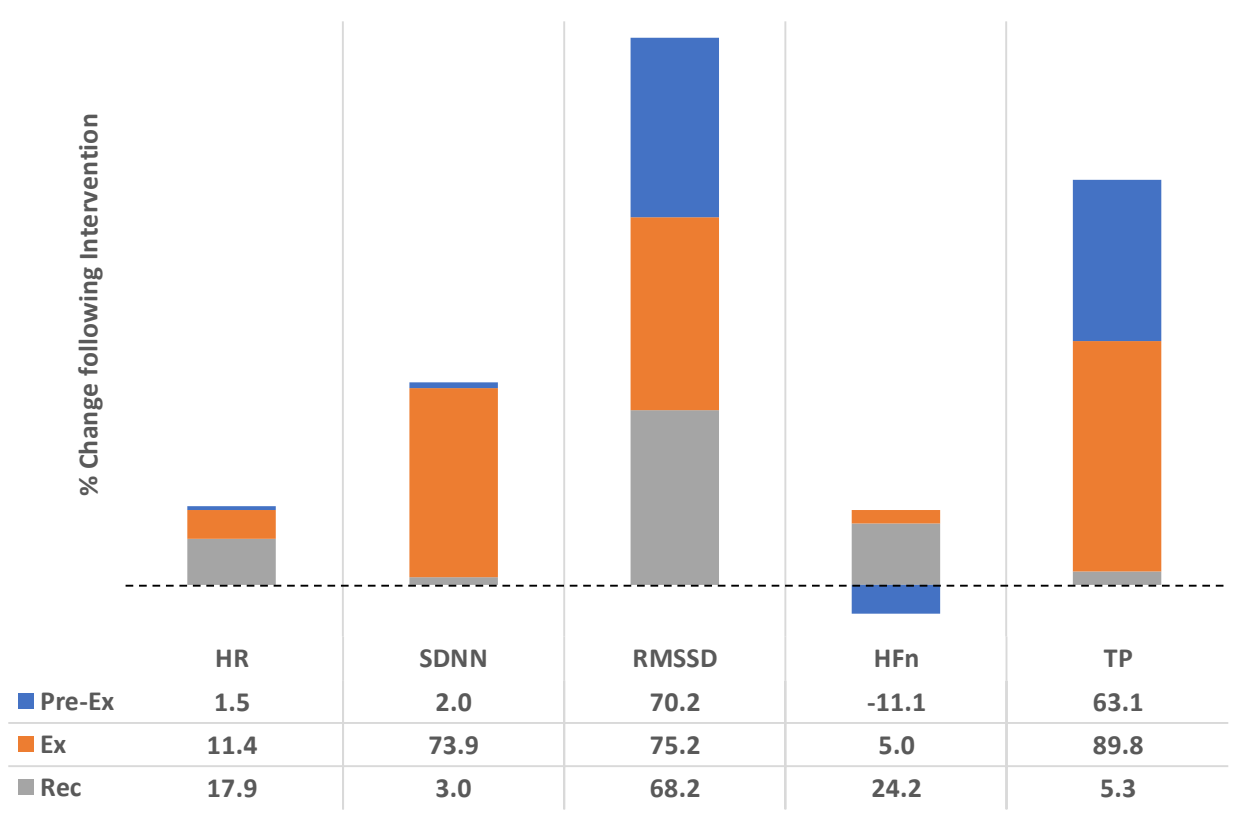

Figure 5.2 Percentage change in HRV indices following PR

\subsubsection{Acceleration and Deceleration Capacity Indices}

To illustrate the PRSA averaging procedure, Figure 5.3 shows the group mean responses (+/- SEM) of the normalized RR time-series around the identified acceleration and deceleration turning-points. Table 5.5 presents the corresponding group mean Acceleration Capacity (AC) and Deceleration Capacity (DC) indices, preand post-rehabilitation. Individual AC and DC values are presented in Appendix C (Table C.5) for each participant, together with the Post-Pre changes in absolute $(\Delta)$ and relative (\%) terms. Figure 5.4 is a box-andwhisker plot of the pre- and post-intervention: the median (lower quartile, upper quartile) AC was -2.7ms (-1.8, -5.3) pre-intervention and $-3.2 \mathrm{~ms}(-2.5,-4.9)$ post-intervention; the median (lower quartile, upper quartile) DC was $2.3 \mathrm{~ms}(1.8,4.8)$ pre-intervention and $3.2 \mathrm{~ms}(2.5,4.3)$ post-intervention. Wilcoxon Signed Rank tests revealed no influence of the intervention on either $A C(p=0.53 ; z=0.63 ; E S=0.11)$ or $D C(p=0.73 ; z=-0.35 ; E S=-$ $0.06)$ indices. 
(a)

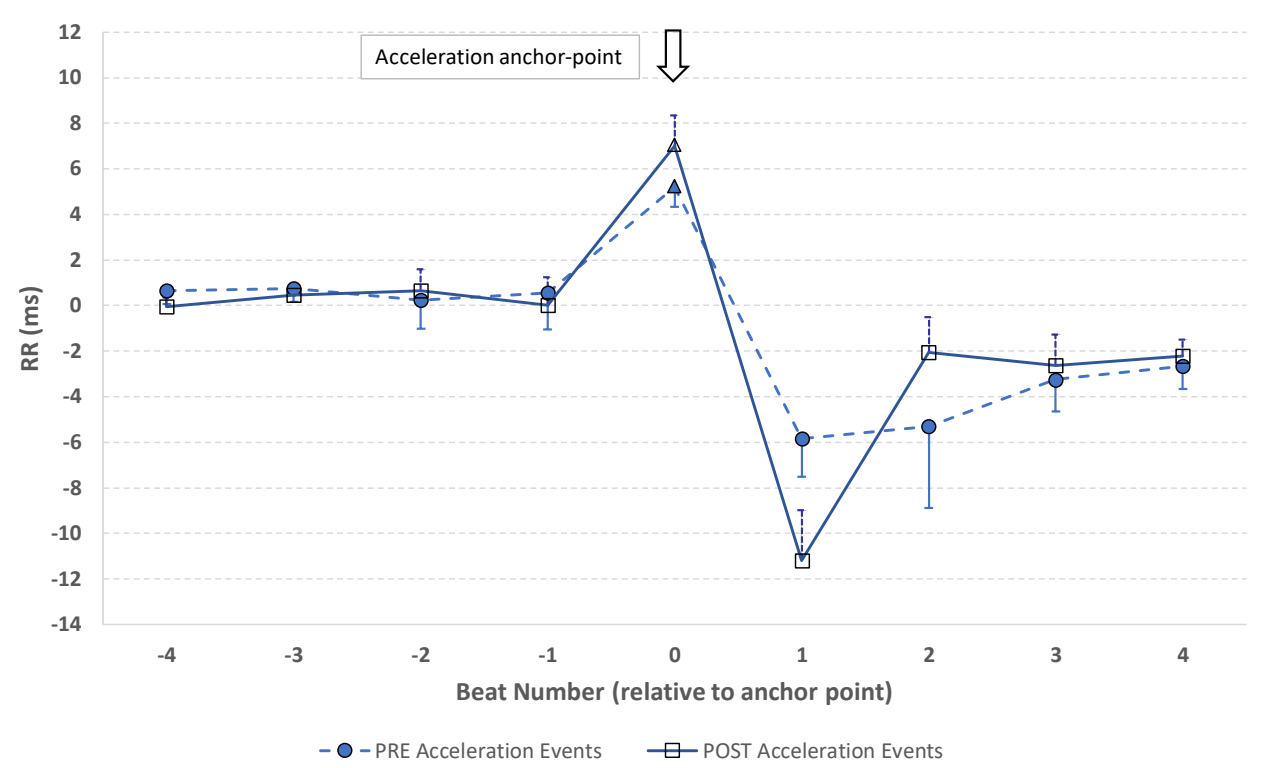

(b)

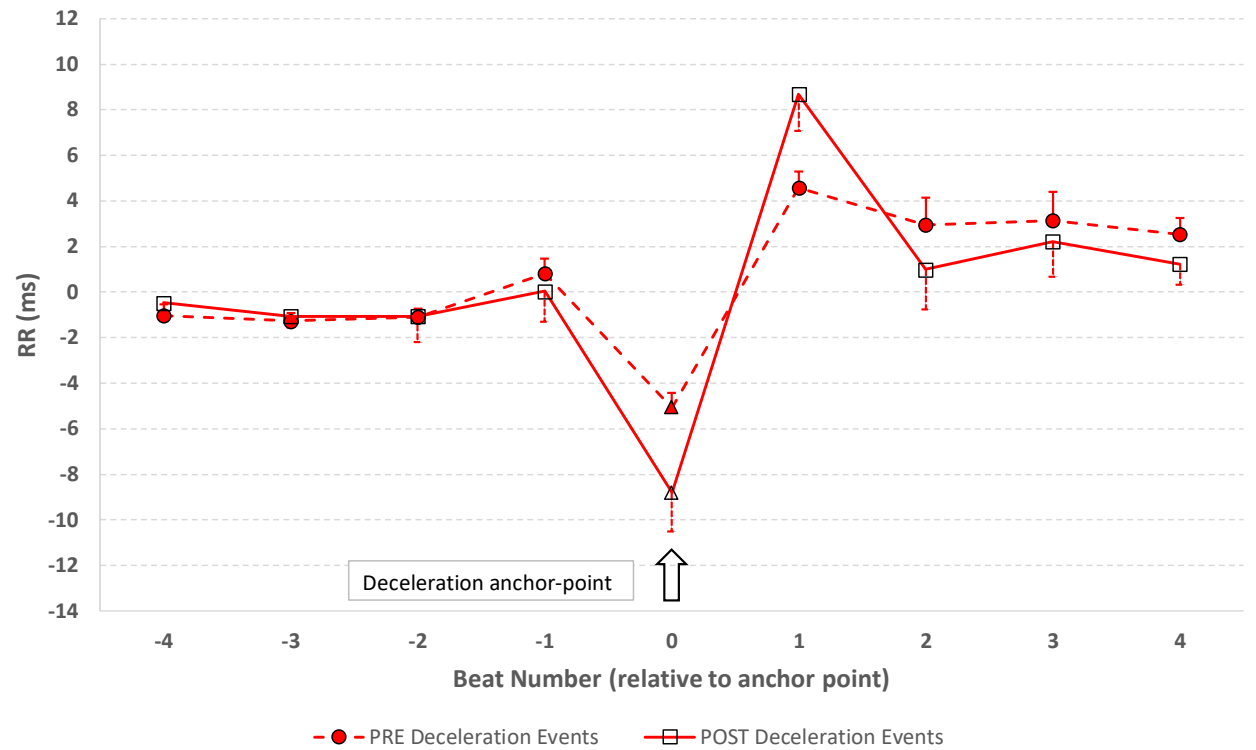

Figure 5.3 Group mean (+/- SEM) of the normalized RR time-series around the identified (a) acceleration and (b) deceleration anchor-points (individual participants' data were normalized by subtracting the mean of the 12 RR intervals immediately preceding the anchor point) 
Table 5.5 summary results for Acceleration Capacity (AC) and Deceleration Capacity (DC) pre and post PR. ( $\Delta$ $(\%)=$ relative change following PR).

\begin{tabular}{cccc}
\hline & Pre-PR: & Post-PR: & \multicolumn{1}{c}{$\Delta(\%)$} \\
& Mean (SD); Range & Mean (SD); Range & \\
\hline AC (ms) & $-4.5(5.1) ;-1.1$ to -18 & $-5.0(5.0) ; 1.2$ to -19.2 & $31.5(61.3) ; 151.1$ to -52.8 \\
DC (ms) & $4.4(4.8) ; 17.2$ to 1.2 & $4.5(4.5) ; 18.2$ to 1.2 & $21.4(56.0) ; 138$ to -39.9 \\
\hline
\end{tabular}

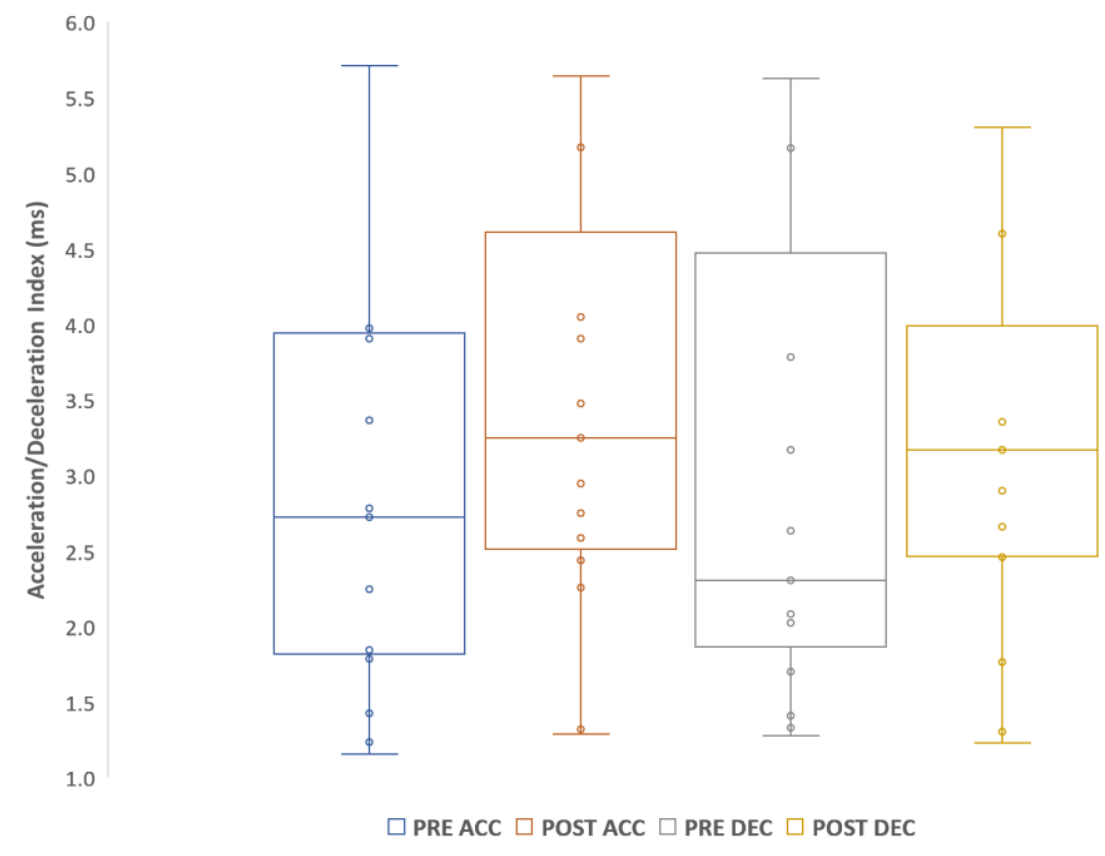

Figure 5.4 Box-and-whisker plot of Acceleration and Deceleration Indices pre (PRE ACC, PRE DEC) and post (POST ACC, POST DEC) PR (absolute values are shown for AC; circles show individual values; horizontal lines in boxes indicate median, lower and upper quartile values; whiskers show range of values) 


\subsection{Discussion}

Traditional measures of pulmonary function (minute ventilation, peak rate of respiratory oxygen uptake) were not influenced by PR. Ramponi et al. [145] reported a 9\% increase in OUES in COPD patients following rehabilitation, so we postulated that the OUES might be a more sensitive measure of aerobic performance plasticity in IPF. However, in this first evaluation of OUES in IPF patients we found that OUES was unchanged following rehabilitation (see Table Appendix C.3).

This was also the first study to investigate the effect of a PR programme on HR responsiveness in people with IPF. We found that HR was elevated by $11-18 \%$ during Exercise and Recovery states post-rehabilitation, suggesting that participants' heart function (heart rate increase in response to exercise) was improved by rehabilitation. Statistically, HRV was not altered by the rehabilitation intervention during any of the three stages of the day 3 protocol (rest, exercise, recovery). However, several substantial relative (post- vs. prerehabilitation) changes were observed that are worthy of note: 1) an increased Pre-Exercise parasympathetic influence (RMSSD elevated by 70\%), 2) increased sympathetic and parasympathetic influences during Exercise (SDNN and RMSSD elevated by 75\%), and 3) an increased parasympathetic influence during Post-Exercise (RMSSD elevated by $68 \%$, HFn elevated by $24 \%$ ). Neither the Acceleration Index nor the Deceleration Index were statistically influenced by the intervention. However, trends for relative (post- vs. pre-rehabilitation) improvements in these indices (changes of $19 \%$ and $38 \%$, respectively) were observed.

The qualitative observation here of altered HRV following physical rehabilitation suggests that this intervention had a positive influence on patients' cardiac autonomic regulation profiles. Conditions of stress (such as moderate physical exercise, a change in posture from supine to standing or mental processing tasks) alter the sympatho-vagal balance towards a more sympathetic-dominant state; this is reflected in frequency-domain HRV by a relative increase in LF power (reduction in HF power) and a reduction in Total Power, with a corresponding relative increase in SDNN (and reduction in RMSSD) in time-domain HRV [101]. During high intensity exercise LF, HF and Total power components are reduced compared with rest and low intensity exercise [146] [99] whereas HF and LF are reduced exponentially during progressive increases in exercise intensity [147] [148] [99]. Furthermore Tulppo et al. [147], Perini et al. [148], and Lewis et al. [100] showed that the decay constants of HRV indices in each bandwidth are linearly related to the maximal work rate. In the present study, the $70 \%$ post-intervention increase in RMSSD during rest suggests that patients' resting cardiac autonomic states became more typical of the relaxed (parasympathetic-dominant) states displayed in healthy people. Increases of similar magnitude (75\%) in RMSSD and SDNN during exercise indicate that patients were 
working at lower relative intensities following the intervention (they found the same activity easier to perform so the exponential-reduction in HRV power was not as great). Finally, following the intervention patients displayed a large (68\%) increase in RMSSD during Recovery, and this faster return to the pre-exercise (parasympathetic-dominant) state likely reflects improved cardiac 'fitness'.

Previous studies on this theme observed significantly reduced values for $A C$ and $D C$ in older compared with younger healthy individuals: Older (mean age 66 years; $A C=-5$ to $-7 \mathrm{~ms} ; \mathrm{DC}=4.5$ to $6.5 \mathrm{~ms}$ ) and Younger (mean age 21 years; $A C=-10$ to $-12 ; D C=8$ to $10 \mathrm{~ms}$ ) [105]. In that study patients with COPD had qualitatively reduced values (AC diminished by 4-6 ms; DC diminished by 2-3 ms) compared with similarly aged healthy people. A further study by colleagues in Swansea added to these data, showing that AC and DC are reduced in middleaged (mean 45 years of age) compared with younger (mean 24 years of age) healthy individuals (mean values $A C=-8.4 /-10.3$ and $D C=7.6 / 9.5 \mathrm{~ms}$, respectively) and that both $A C$ and $D C$ are enhanced in aerobically superior individuals [149]. The AC and DC values observed for patients with IPF in the present study (AC = -2.7 to -3.2 $\mathrm{ms} ; \mathrm{DC}=2.3$ to $3.2 \mathrm{~ms}$ ) are around $50 \%$ lower than those of similarly aged healthy people and are similar to those of individuals with COPD who have not received oxygen therapy. Visualisation of the RR time-series around the identified acceleration and deceleration anchor-points enabled the following observations: 1) acceleration anchor-points occurred at larger RR values after rehabilitation, 2) deceleration anchor-points occurred at smaller RR values after rehabilitation, 3) immediately post-anchor acceleration was greater (RR values achieved were smaller) after rehabilitation, and 4) immediate post-anchor deceleration was greater (RR values achieved were larger) after rehabilitation. These observations mean that, following rehabilitation: 1) heart rate was allowed to reach lower values before triggering a larger tachycardic response and 2) heart rate was allowed to reach higher values before triggering a larger bradycardic response. We suggest that these results provide evidence that the physical rehabilitation intervention may have improved the dynamic range of heart rate responsiveness in our patients with UIP. We further speculate that this would have enhanced patients' physical function during everyday tasks (being mindful that the $A C$ and $D C$ indices were calculated as an ensemble across each of the three physiological states in our protocol).

The diminished cardiac acceleration and deceleration capacities in IPF patients reflect the autonomic dysfunction that is also indicated qualitatively by the HRV variables for this group of individuals. Postintervention improvements in AC and DC indices appear to reflect the observed shift towards 'healthy' HRV values for these patients. Taken together, these changes suggest that physical rehabilitation had a positive 'normalising' influence on cardiac autonomic modulation of heart rate in people with UIP. 


\subsection{Limitations of the study}

Whilst we consider our sample of 15 patients to be representative of people with UIP (IPF), this relatively small number of participants reduces the statistical power of our analyses. The study also excluded patients who had co-morbid conditions that might influence cardiac or autonomic nervous system function. Whilst this removes the potential confounding influence of other pathologies, it reduces our ability to generalise our results to the wider population of people with UIP (IPF). We also chose not to perform sub-group analyses, for example to examine the impact of age, gender, and disease stage or medication regime on the measured physiological variables. Such analyses would require a much larger study population and would presumably offer useful insight into the relative efficacy of PR programmes for defined sub-groups of the IPF population.

\subsection{Strengths of the study}

At the beginning of the study we were mindful of the potential influence of too strict a set of exclusion criteria on both recruitment and the applicability of our results to a wider population of people with UIP. With this in mind we took the decision to not exclude individuals with controlled diabetes from the study: five (27\%) of our patient cohort were taking medication to treat Type II diabetes. Furthermore, patients acted as their own controls and care was taken to ensure that the only change between the monitoring days was the intervening rehabilitation intervention. Patients' drug regimens were not changed during the study, so the results reflect patients' 'usual' health status. This study demonstrated the feasibility of employing a demanding physiological assessment protocol in this acutely unwell population. The results will enable sample-size calculations for appropriately-powered statistical evaluations in future, larger-scale studies.

\subsection{Concluding comments}

To our knowledge this is the first study of its kind to use both HRV and PRSA analyses to assess the outcomes of a physical rehabilitation programme. The results of this study provide initial evidence that physical rehabilitation improves heart rate dynamics (via modulation of autonomic control of heart rate) in IPF patients, even when respiratory and physical functions are unchanged or diminished. 


\section{Chapter 6}

\section{Heart rate kinetics and respiratory responses to extreme (rapid-onset, high workload) metabolic challenge}

\subsection{Overview}

The 'oxygen uptake efficiency slope' quantifies the body's oxygen uptake and extraction efficiency (the rate of increase in oxygen uptake $\left(\mathrm{VO}_{2}\right)$ in response to increases in minute ventilation (VE) during incremental exercise. The OUES has several advantages over other measures of aerobic exercise performance: it can be determined accurately from submaximal exercise protocols (below 60-75\% functional reserve) [17] [18] [20] yet is strongly correlated with $\mathrm{VO}_{2}$ max; it is completely objective (tester-independent) and it has better inter-protocol agreement [17] [18]. An alternative to OUES is the analysis of so-called 'pulmonary oxygen uptake kinetics' which involves mathematical modelling of the rate of change of oxygen utilisation in response to a sudden change in exercise workload. This provides insight into an individual's ability to absorb and utilize oxygen to fuel a change in metabolic activity [23]. The procedure involves fitting either a mono-exponential or bi-exponential model to the empirical temporal data, with the time-constant of the best-fit model reflecting an individual's oxygen utilisation response rate. This chapter reports the findings of investigations into the influence of an eight-week, exercise-based PR programme on dynamic pulmonary function (oxygen uptake kinetics) and cardiac function (heart rate kinetics)

\subsection{Results}

\subsection{1 $\mathrm{VO}_{2}$ responses to maximal/incremental and constant workload exercises}

In this section a summary of the procedure for determining the individual constant work rate values used in the day 3 protocol is first presented. Figure 6.1 shows a typical ${ }^{\mathrm{V}} \mathrm{O}_{2}$ response to the maximal incremental exercise (day 2) protocol for an individual participant. The gas exchange threshold (GET), also known as the aerobic threshold (AT) was determined as the $\dot{\mathrm{VO}}_{2}$ value where a nonlinear increase in $\mathrm{CO}_{2}$ production $\left(\mathrm{VCO}_{2}\right)$ relative to $\mathrm{VO}_{2}$ was observed together with an increase in minute ventilation $(\mathrm{VE}) / \mathrm{VO}_{2}$ without an increase in $\mathrm{VE} / \mathrm{V} \mathrm{O}_{2}$ [16]. The workload intensity for the constant work rate test was calculated as the resistance that elicits a $\mathrm{VO}_{2}$ of $40 \%$ of the difference between the GET and the peak $\dot{\mathrm{VO}}_{2}(\Delta 40 \%)$ [16]. Figure 6.2 shows a typical $\dot{\mathrm{VO}}_{2}$ response to the constant workload (day 3) protocol for the same individual. Data from the interval 'Start of constant work rate' to 'Start of Recovery' were extracted and used for subsequent analysis (modelling) of the $\mathrm{VO}_{2}$ response (Section 6.2.4). 


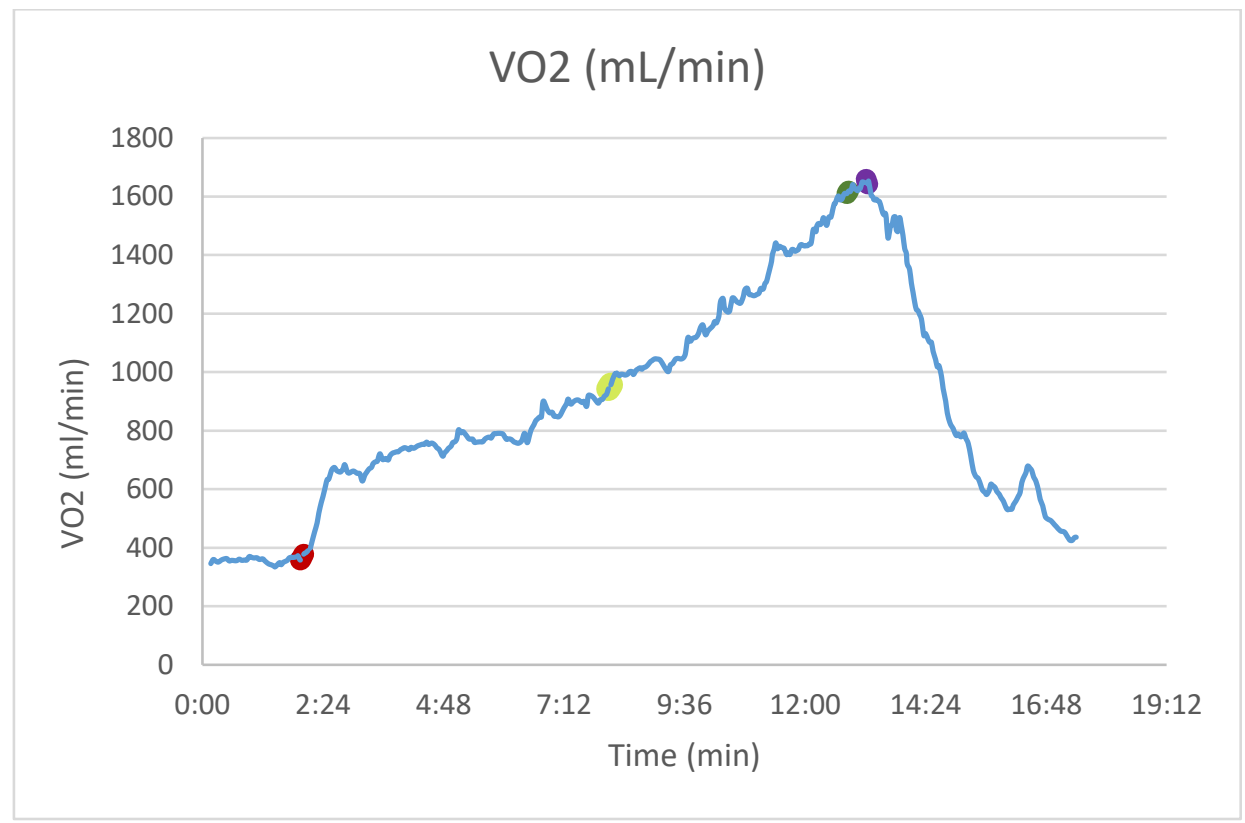

Figure 6.1: Example of an individual $\mathrm{V}_{2}$ response during the pre-exercise (starting at the red point), exercise (red-to-green) and post-exercise phases (starting at the purple point) of the day 2 protocol (maximum work rate test). The Gas Exchange Threshold is represented by the yellow point.

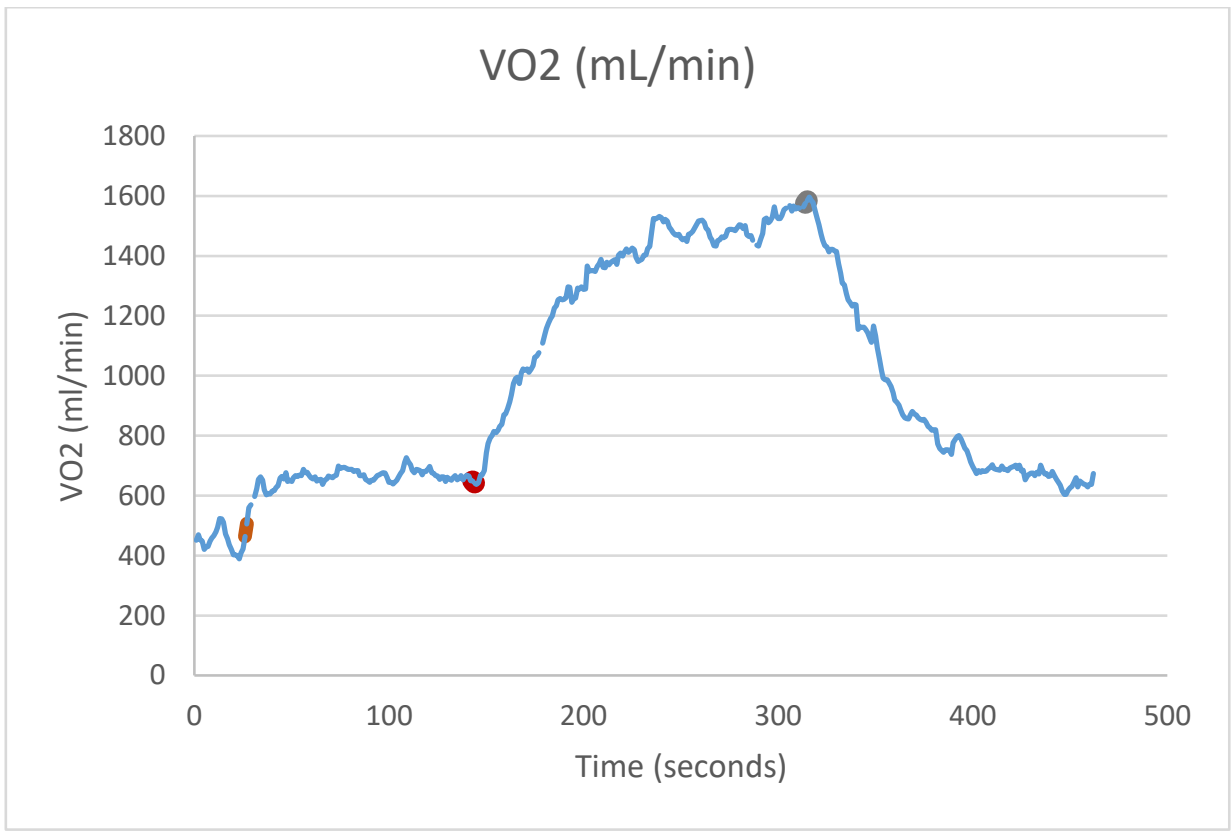

Figure 6.2: Example of an individual $\mathrm{VO}_{2}$ response during the pre-exercise, exercise and post-exercise phases of the day 3 protocol (constant work rate test) (no phase 1 or phase 3 changes were observed). The orange point indicates start of exercise, red indicates the start of the constant work rate test protocol, and green represents the start of the recovery period. 
6.2.2 Responses to the constant work rate (day 3) exercise protocol

Table 6.1 summarises the physiological responses to constant work rate exercise tests, pre- and postrehabilitation. $\mathrm{VE}$, the highest recorded $\mathrm{VO}_{2}$ and the highest recorded heart rates were all unchanged following PR.

Table 6.1: Physiological responses to constant workload tests (Day 3 protocol), pre- and post-rehabilitation (these values are reported for twelve patients only, owing to equipment malfunction and the absence of pre/post-PR data for two participants.)

\begin{tabular}{l|lll}
\hline \multicolumn{1}{c}{$\begin{array}{l}\text { Pre-PR } \\
\text { Mean (SD); Range }\end{array}$} & $\begin{array}{l}\text { Post-PR } \\
\text { Mean (SD); Range }\end{array}$ & \\
\hline $\mathrm{VE}\left(\mathrm{m} / \cdot \mathrm{min}^{-1} \cdot \mathrm{kg}^{-1}\right)$ & $54.0(15.8) ; 30.3-87.1$ & $48.1(18.5) ; 21.6-78.8$ & 0.25 \\
Highest $\dot{\mathrm{VO}} 2\left(\mathrm{~L} \cdot \mathrm{min}^{-1}\right)$ & $1.27(0.29) ;(0.85-1.81)$ & $1.17(0.35) ;(0.63-1.90)$ & 0.21 \\
Highest $\dot{\mathrm{VO}} 2\left(\mathrm{~m} / \cdot \mathrm{min}^{-1} \cdot \mathrm{kg}^{-1}\right)$ & $15.0(4.6) ; 9.1-28.1$ & $13.8(4.9) ; 6.4-25.6$ & 0.35 \\
Highest HR $(\mathrm{bpm})$ & $117(22) ;(90-151)$ & $120(26) ; 70-148$ & 0.70 \\
\hline
\end{tabular}

Figure 6.3(a) shows the $\mathrm{V}_{2}$ responses to constant workload exercise for a selection of participants (prior to $\mathrm{PR}$ ). Individual responses were then normalized to the end-exercise value: all breath-by-breath $\mathrm{V}_{2}$ values were divided by the maximum $\mathrm{V}_{2}$ value (determined within the last 30 seconds of exercise) for that individual (Figure 6.3(b)). Figures 6.4(c) and (d) show the equivalent data for these participants following PR. 
Figure 6.3: (a) Individual $\mathrm{VO}_{2}$ responses to constant workload exercise for a selection of participants before PR (data are shown for 12 individuals); (b): Normalised individual $\mathrm{VO}_{2}$ responses for the same participants as in (a); (c): individual $\dot{\mathrm{V}}_{2}$ responses to the constant workload test, post PR; (d): normalised $\mathrm{VO}_{2}$ responses for the same participants as in (c).

(a)

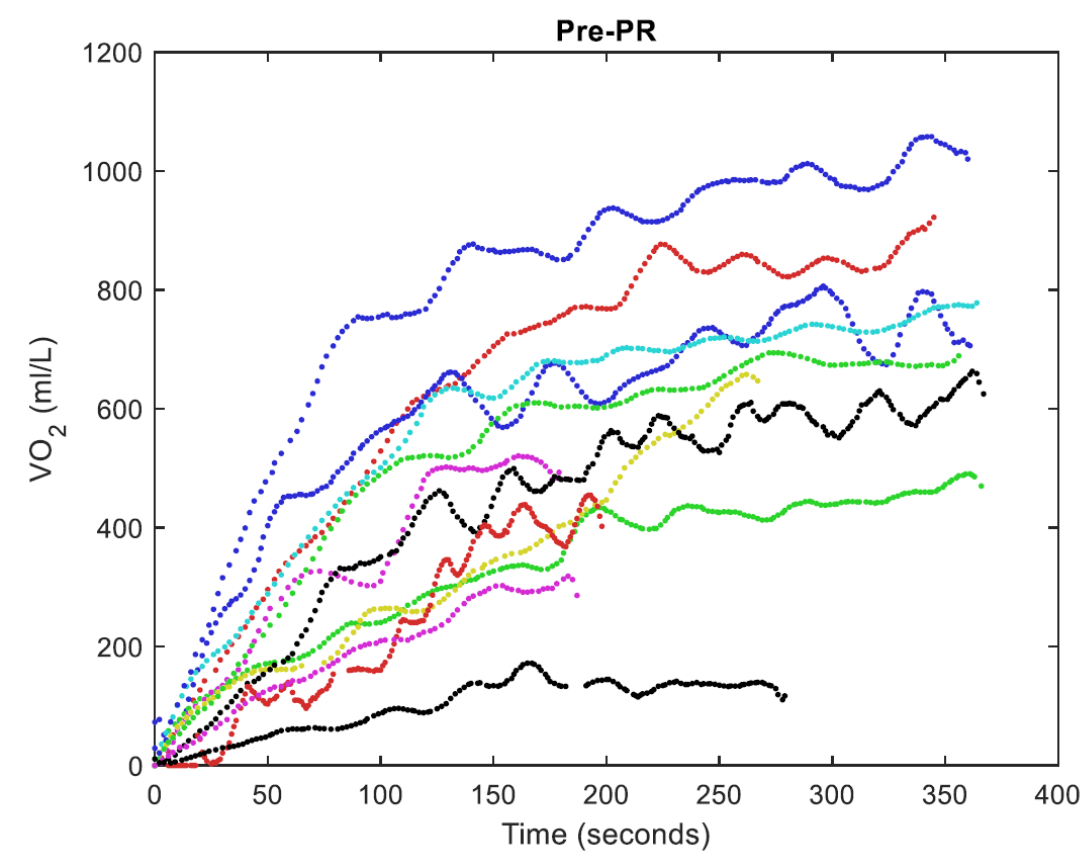

(b)

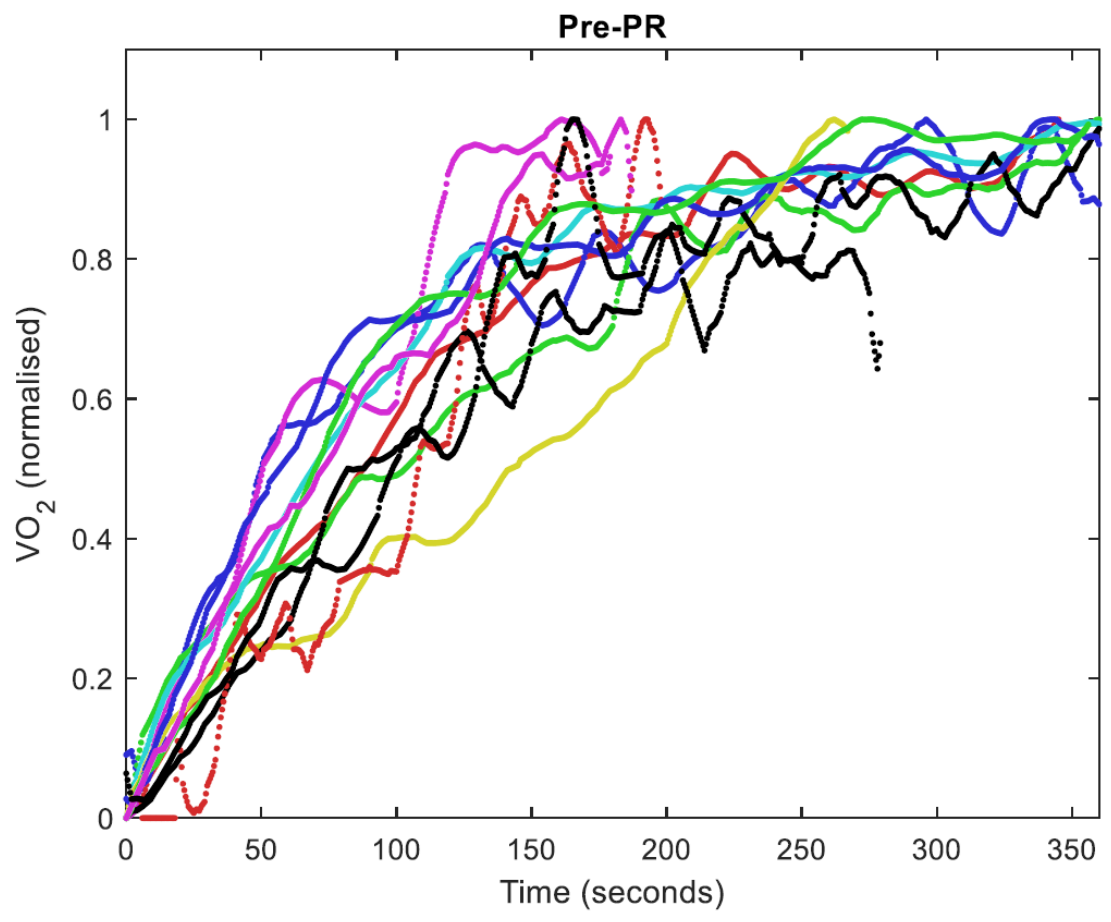


(c)

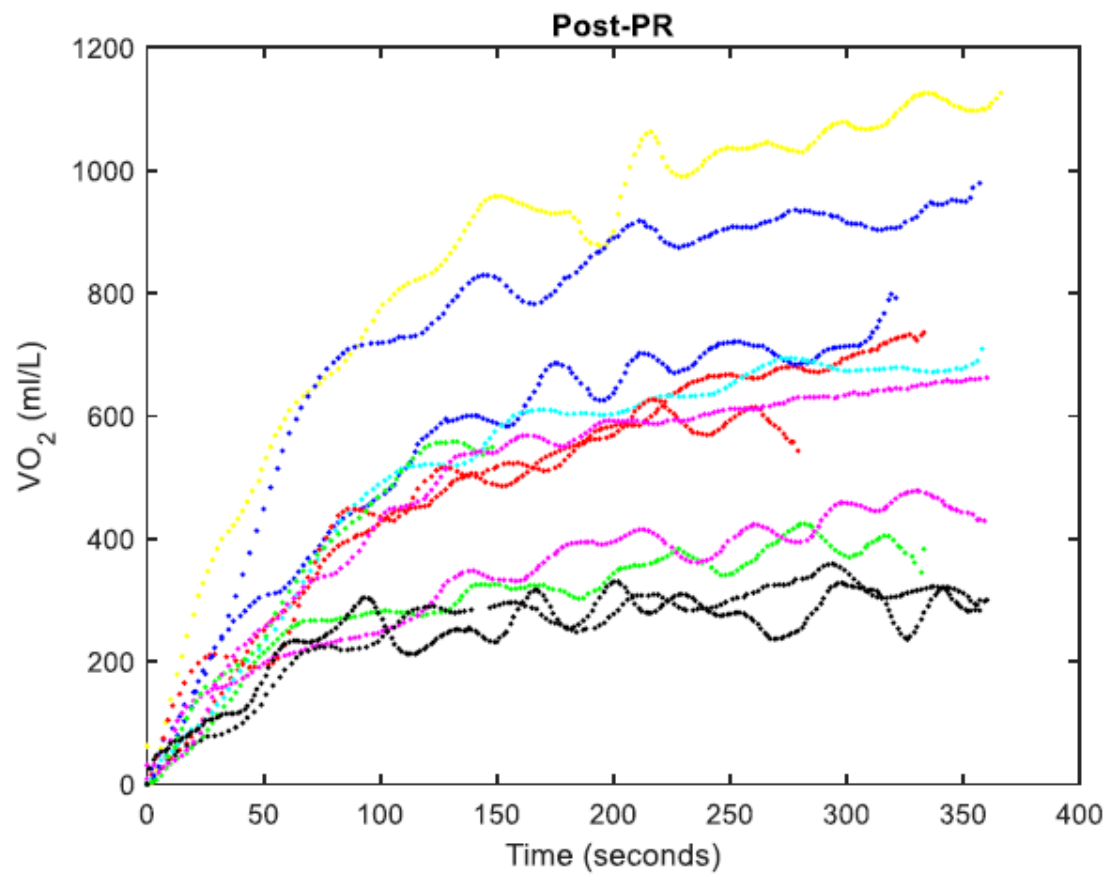

(d)

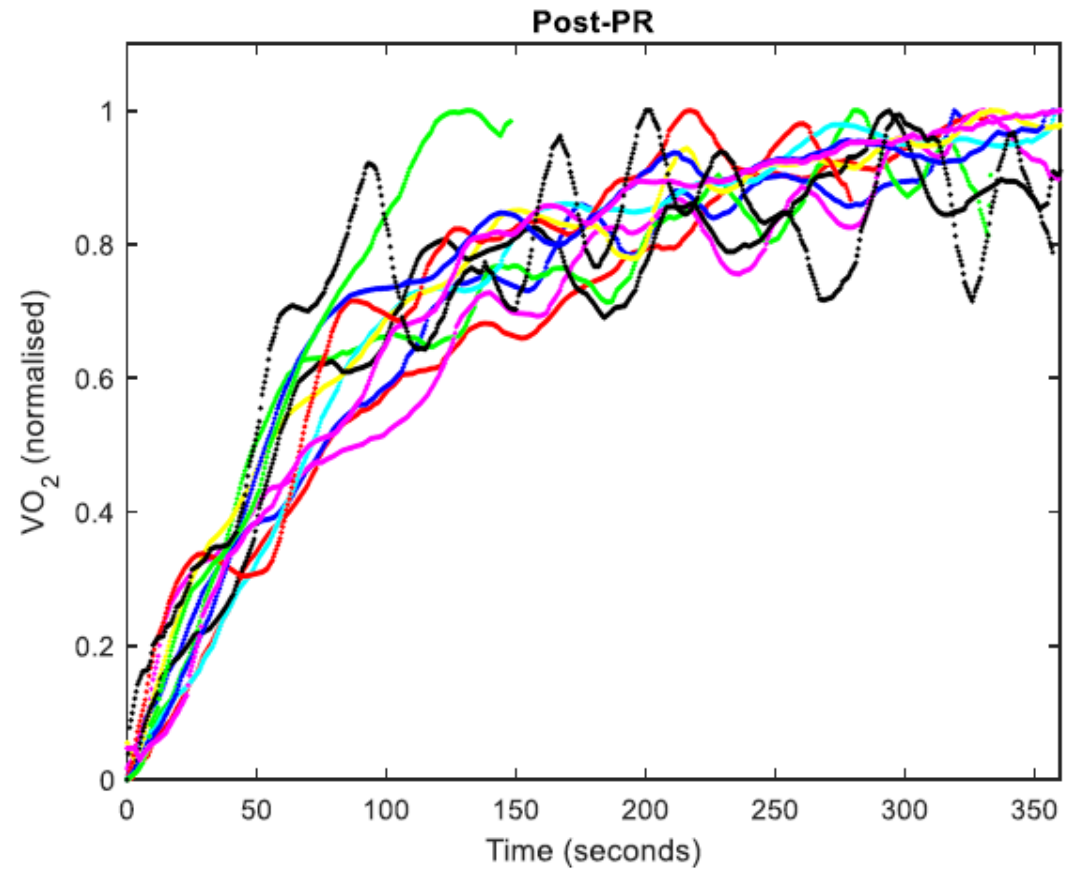




\subsubsection{Heart rate responses to constant workload exercise}

Figure 6.4 (a) shows the HR responses to constant workload exercise for a selection of participants pre rehabilitation. Individual responses were then normalized to the end-exercise value: all HR values were divided by the maximum HR value (determined within the last 30 seconds of exercise) for that individual (Figure 6.4(b)). Figures $6.4(\mathrm{c})$ and $(\mathrm{d})$ show the equivalent data for these participants following PR. Figure 6.5(a) shows the group mean HR responses to constant workload exercise for pre and (b) post PR. 
Figure 6.4: (a) Individual HR responses to constant workload exercise for a selection of participants (data are shown for 05 individuals) after PR; (b): Normalised individual HR responses for the same participants as in (a); (c): individual HR responses to the constant workload test, post PR; (d): normalised HR responses for the same participants as in (c).

(a)

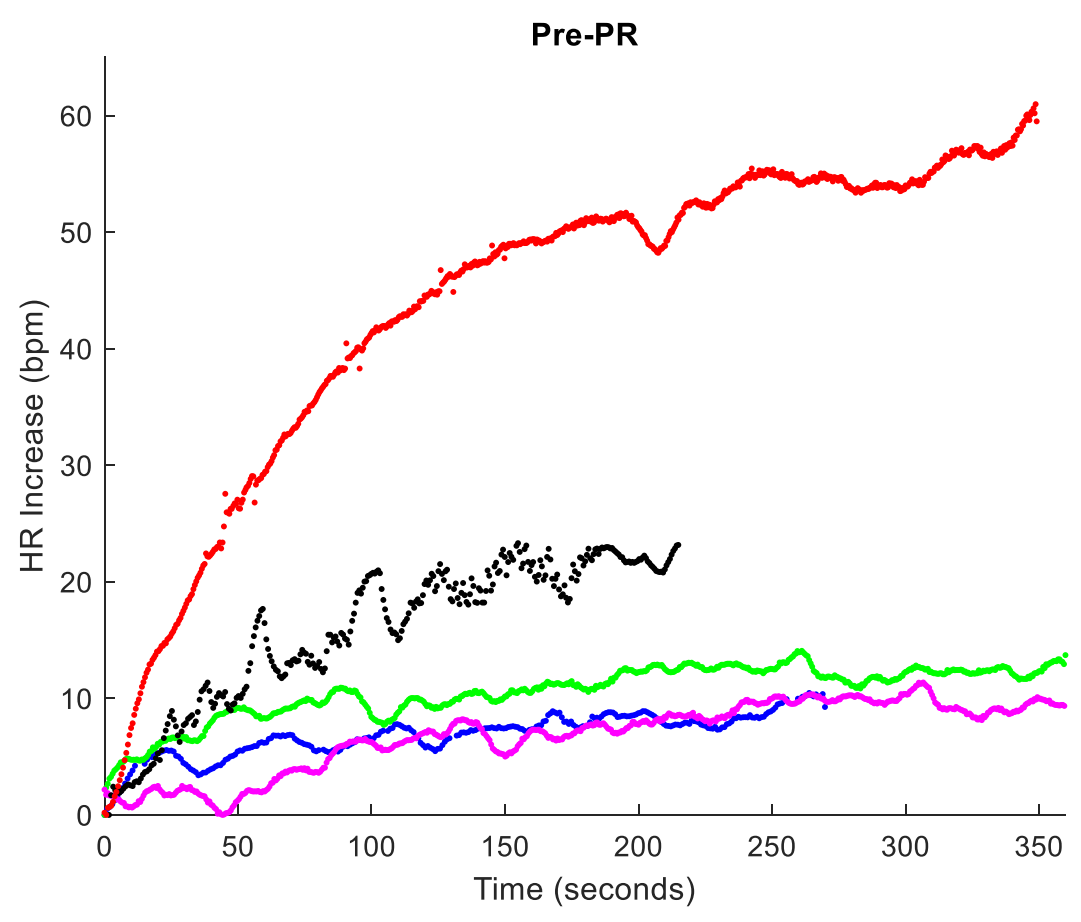

(b)

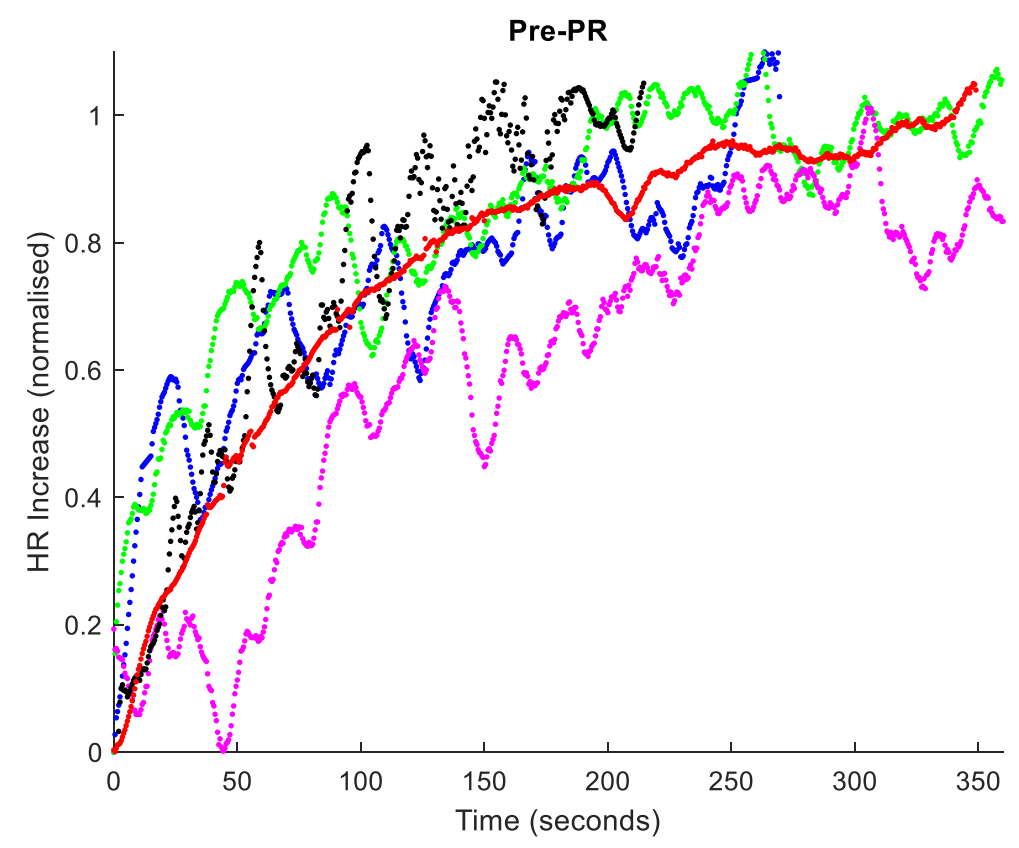


(c)

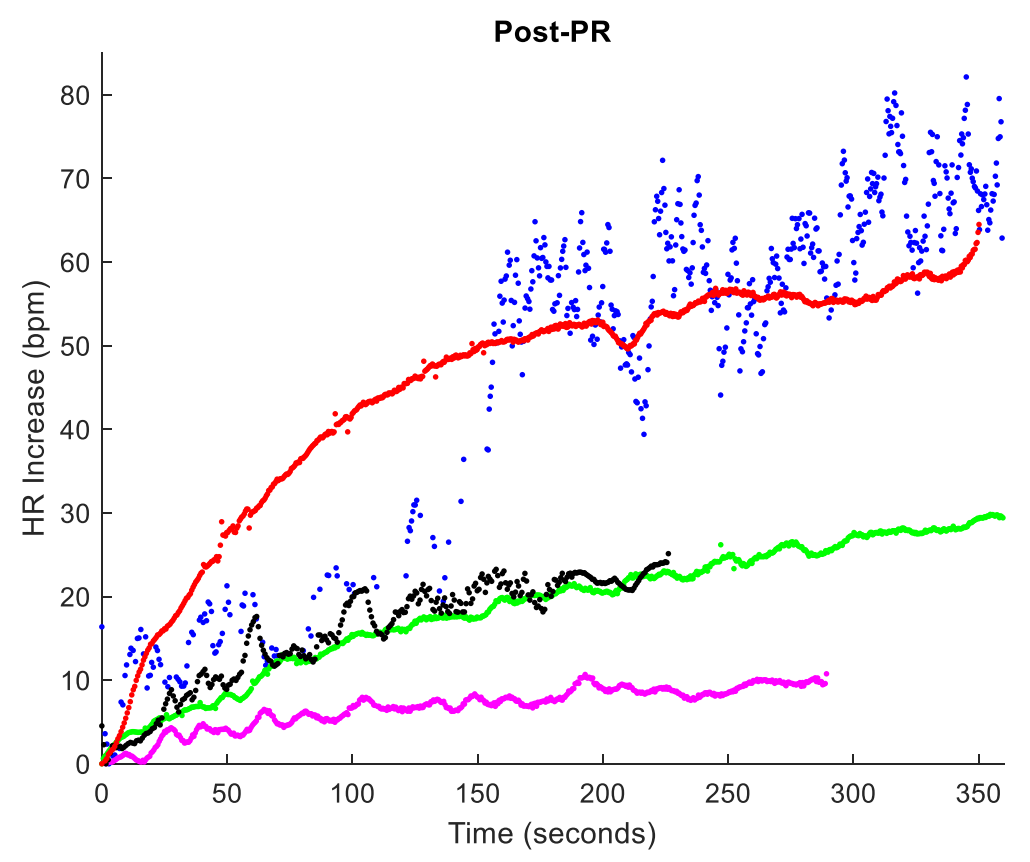

(d)

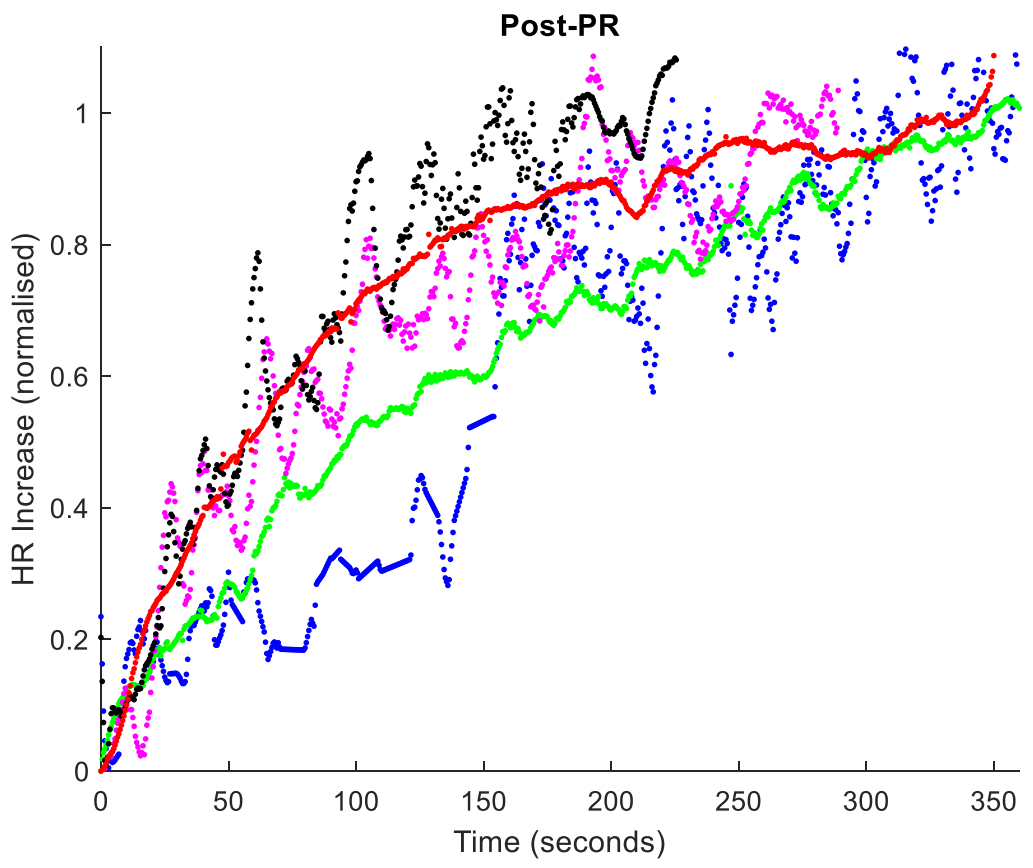


6.2.4 Modelling the $\dot{\mathrm{VO}}_{2}$ and heart rate responses to constant workload exercise

Figure 6.5 shows the curve fitting process (using the Matlab curve fitting toolbox, as described in Sections 3.2.5.4-3.2.5.7) used to model the group mean $\dot{\mathrm{V}}_{2}$ data ( $a$; pre-PR, $b$; post-PR). The same process was used to model the HR data (Figure 6.6: a; Pre-PR, b; post-PR). Table 6.2 summarises the results of curve fitting for the participant group. Curve fitting results for individual participants' $\mathrm{V}_{2}$ and $\mathrm{HR}$ responses are shown in Appendix D.1 and Appendix D.2.

Table 6.2: Results of group-averaged, normalised $\dot{\mathrm{VO}}_{2}$ and $\mathrm{HR}$ modelling

\begin{tabular}{c|cccc}
\hline \multirow{2}{*}{ Model } & \multicolumn{2}{c}{$\mathrm{VO}_{2}$} & \multicolumn{2}{c}{$H R$} \\
\cline { 2 - 5 } Attribute & Pre-PR & Post- & Pre-PR & Post- \\
& $\mathbf{( 9 5 \%}$ & $\mathbf{P R}$ & $\mathbf{( 9 5 \%}$ & $\mathbf{P R}$ \\
& $\mathbf{C l})$ & $\mathbf{( 9 5 \%}$ & $\mathbf{C l})$ & $\mathbf{( 9 5 \%}$ \\
& & $\mathbf{C l})$ & & $\mathbf{C l})$ \\
A (nu) & 0.994 & 0.956 & 0.97 & 1.022 \\
& $(0.990$, & $(0.954$, & $(0.964$, & $(1.014$, \\
& $0.998)$ & $0.959)$ & $0.976)$ & $1.029)$ \\
MRT & 107.9 & 85.2 & 60.5 & 115.5 \\
(sec.) & $(106.6$, & $(84.4$, & $(58.9$, & $(113.2$, \\
& $109.1)$ & $86.0)$ & $62.0)$ & $117.8)$ \\
SSE & 0.287 & 0.226 & 1.862 & 0.822 \\
$R^{2}$ & 0.994 & 0.995 & 0.940 & 0.983 \\
$R M S E$ & 0.020 & 0.018 & 0.051 & 0.034 \\
\hline
\end{tabular}

SSE = Summed square of residuals (sum of squares due to error); R-squared = squared correlation (coefficient of multiple determination); RMSE = root mean squared error (standard error of the fit); nu = normalised units 
(a)

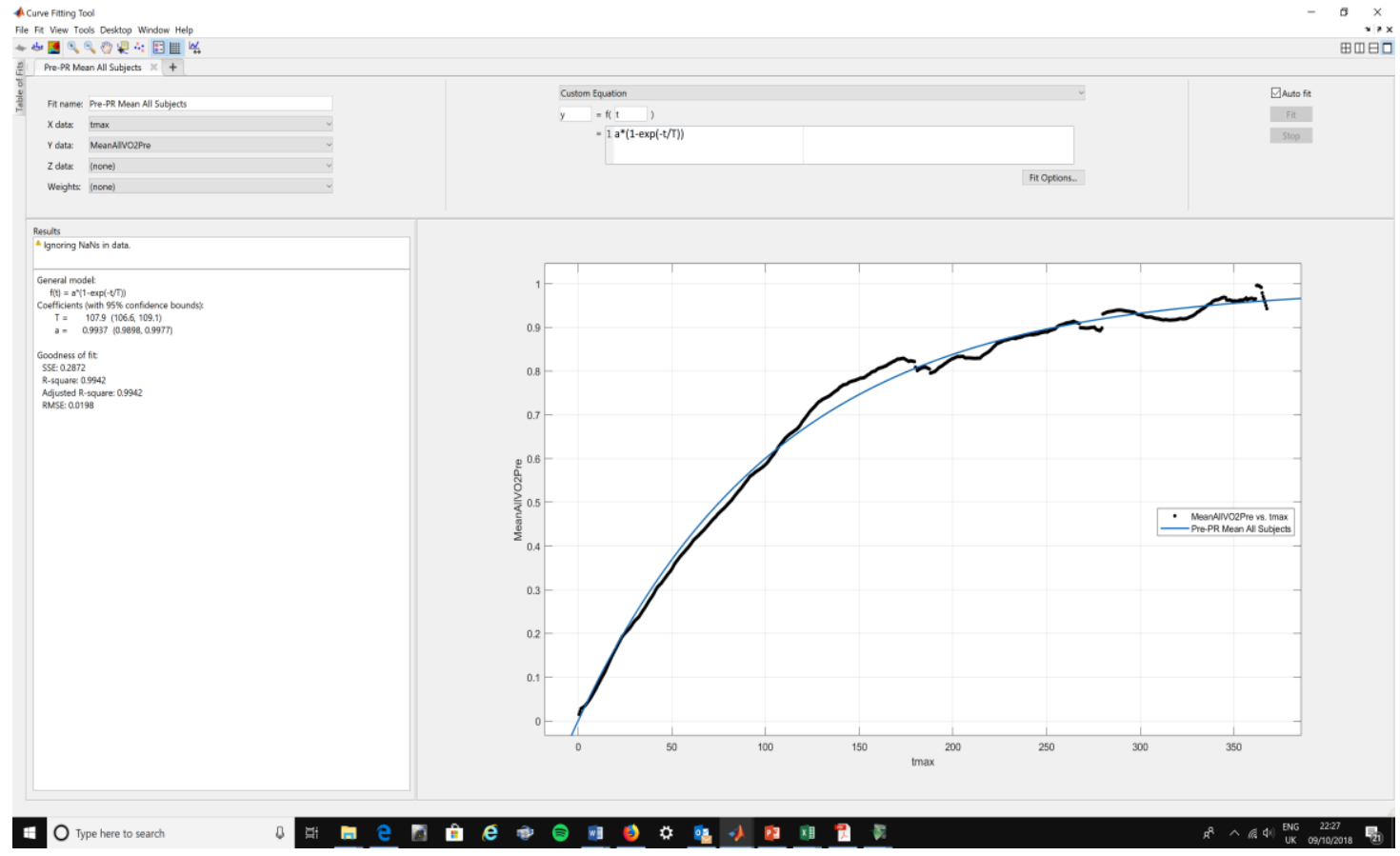

(b)

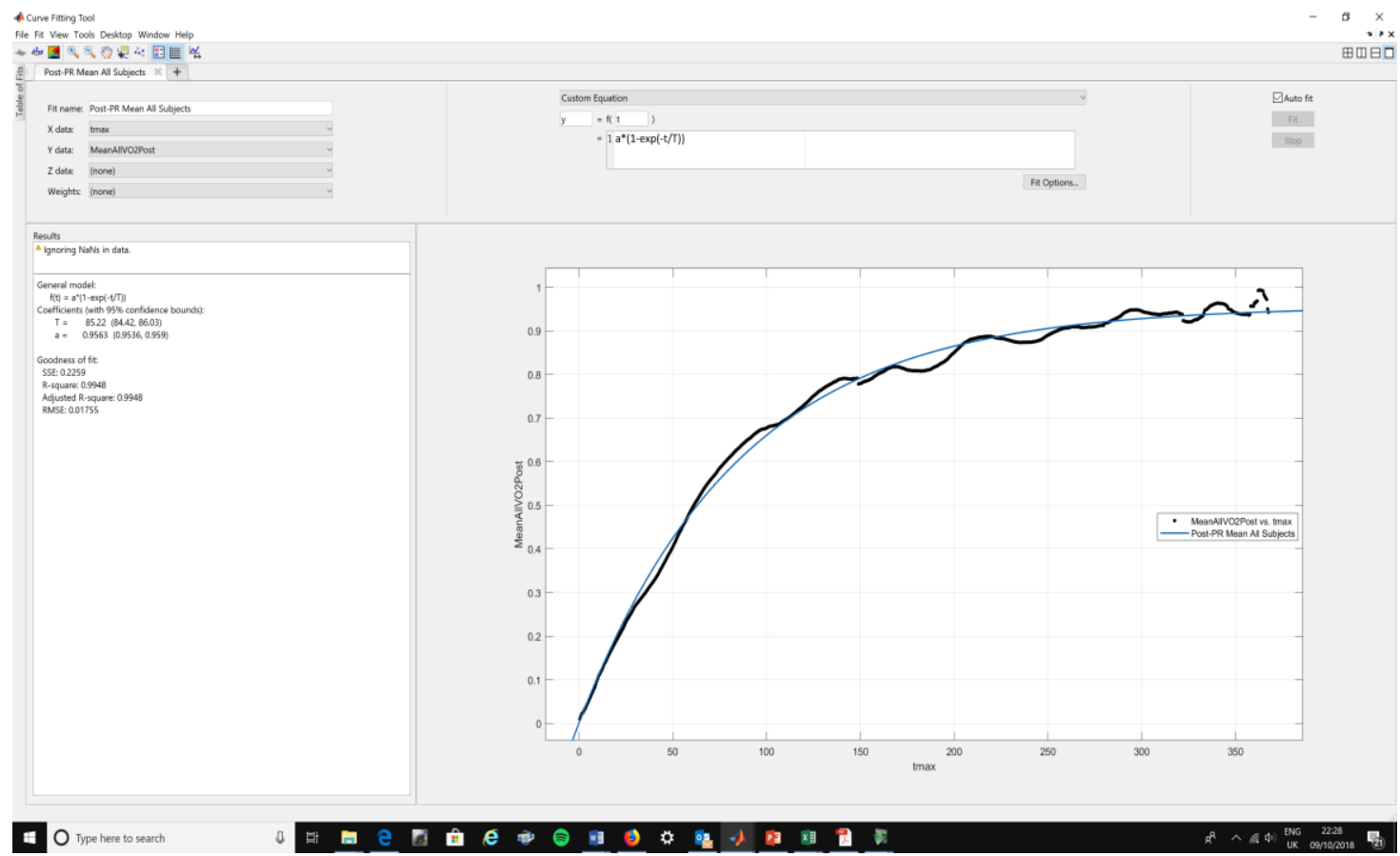

Figure 6.5 The curve fitting process used to model the group mean $\dot{\mathrm{VO}}_{2}$ data (Matlab curve fitting toolbox): (a) Pre-PR and (b) Post-PR 
(a)

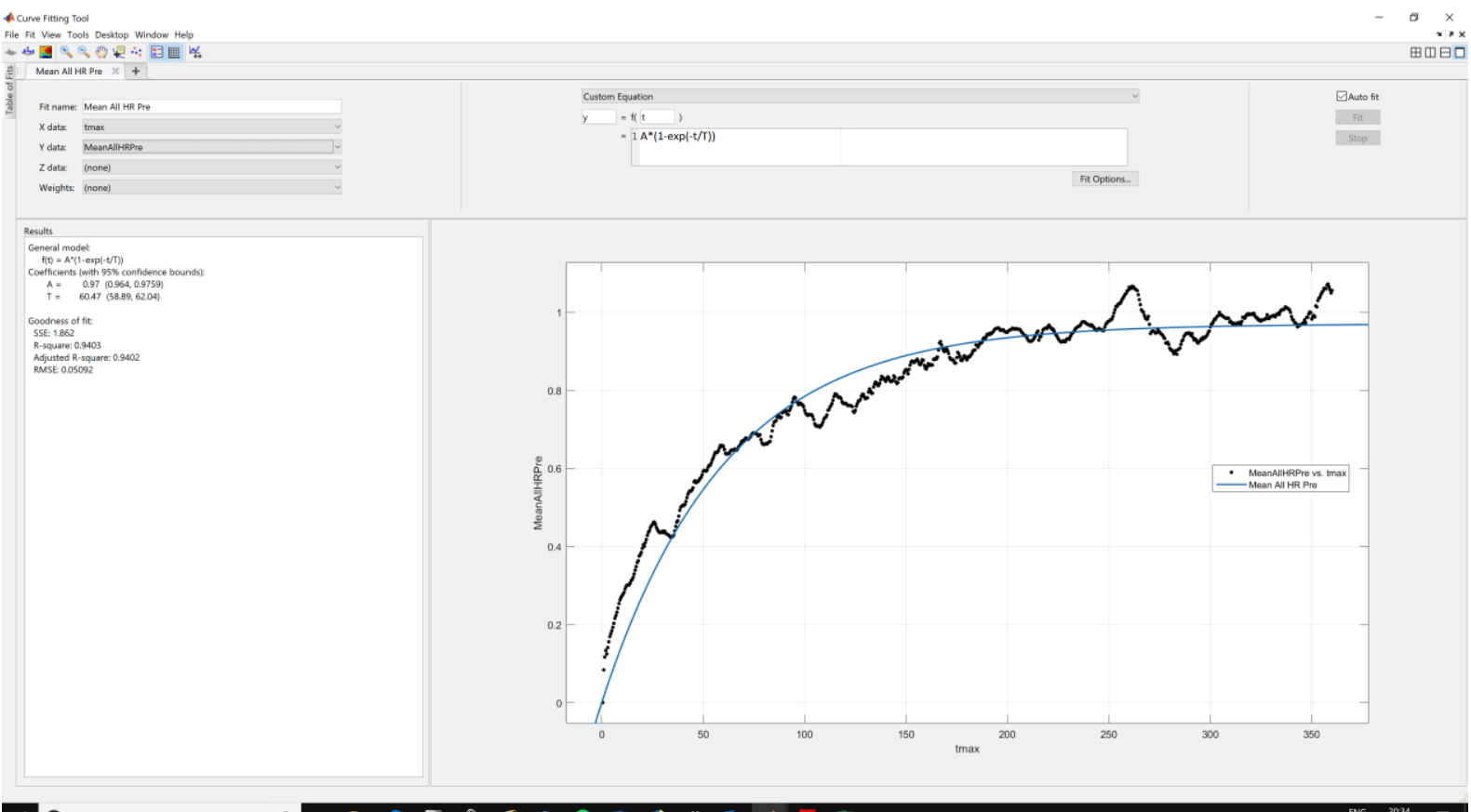

(b)

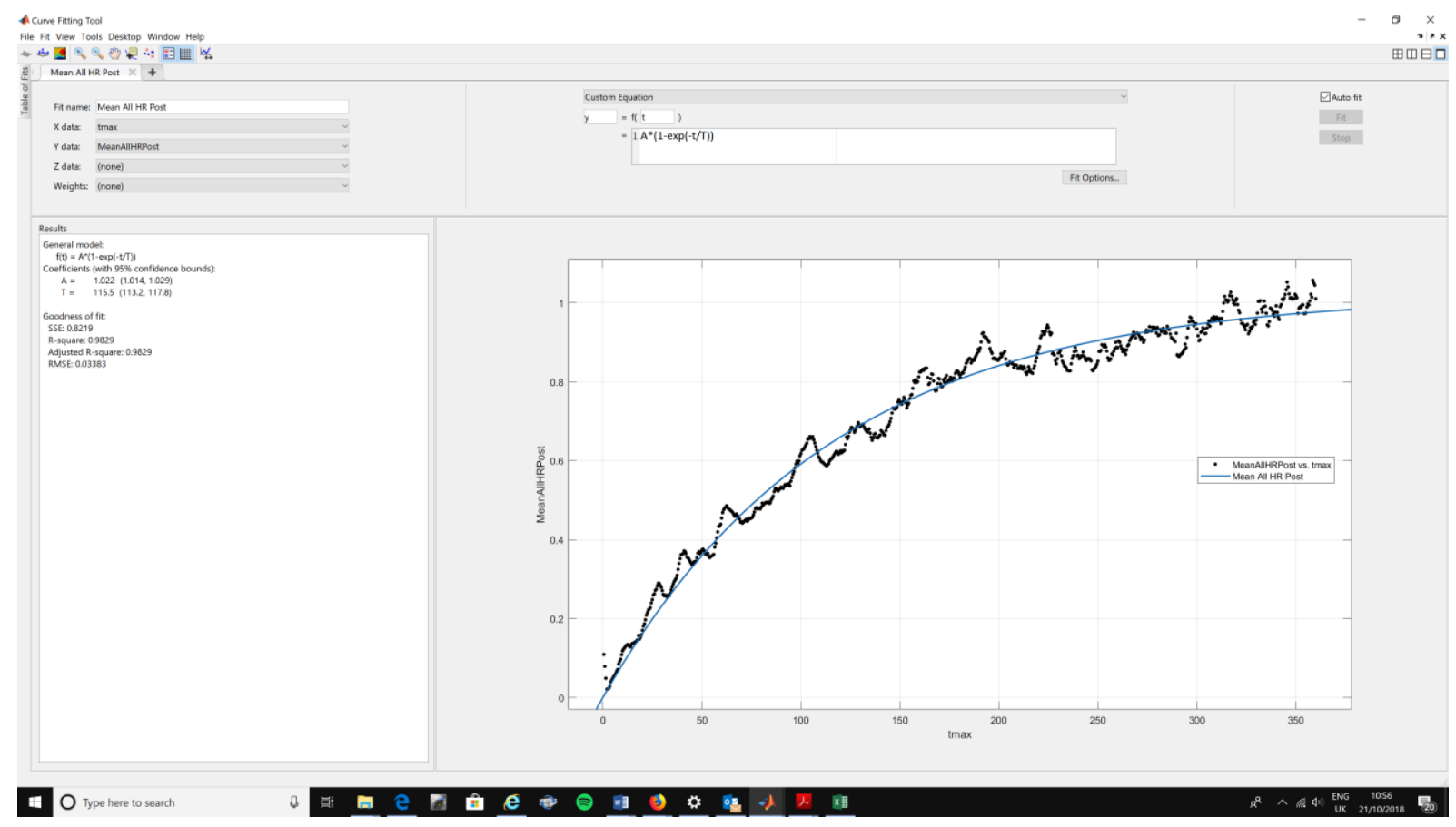

Figure 6.6 The curve fitting process used to model the group mean HR data (Matlab curve fitting toolbox): (a) Pre-PR and (b) Post-PR 
Figure 6.7 shows the group-averaged, normalised temporal $\dot{\mathrm{V}}_{2}$ response to constant work rate exercise. Visual inspection of Figure 6.7 (a) suggested an increase in $\mathrm{VO}_{2}$ post-rehabilitation during the first two minutes of exercise. Statistical comparison (Wilcoxon signed rank test of $\dot{\mathrm{VO}}_{2}$ at each of the 12 time-points in the resampled time-series; Figure 6.7 (b)) confirmed that $\mathrm{VO}_{2}$ was significantly greater post-rehabilitation at times $\mathrm{t}$ $=60,90$ and 120 seconds ( $p=0.028,0.008$ and 0.034 respectively).

Visual inspection of the group-averaged HR responses during the constant work rate tests (Figure 6.8(a)) suggested a reduction in HR post-rehabilitation during the first four minutes of exercise. Statistical comparison (Wilcoxon signed rank test of HR at each of the 12 time-points in the re-sampled time-series; Figure 6.8 (b)) confirmed that HR was significantly lower post-rehabilitation at times $t=30,120$ and 210 seconds $(p$ values $=$ 0.043 ) and approached a significant difference at times $t=90,180$ and 240 seconds ( $p$ values $=0.079$ ). 
(a)

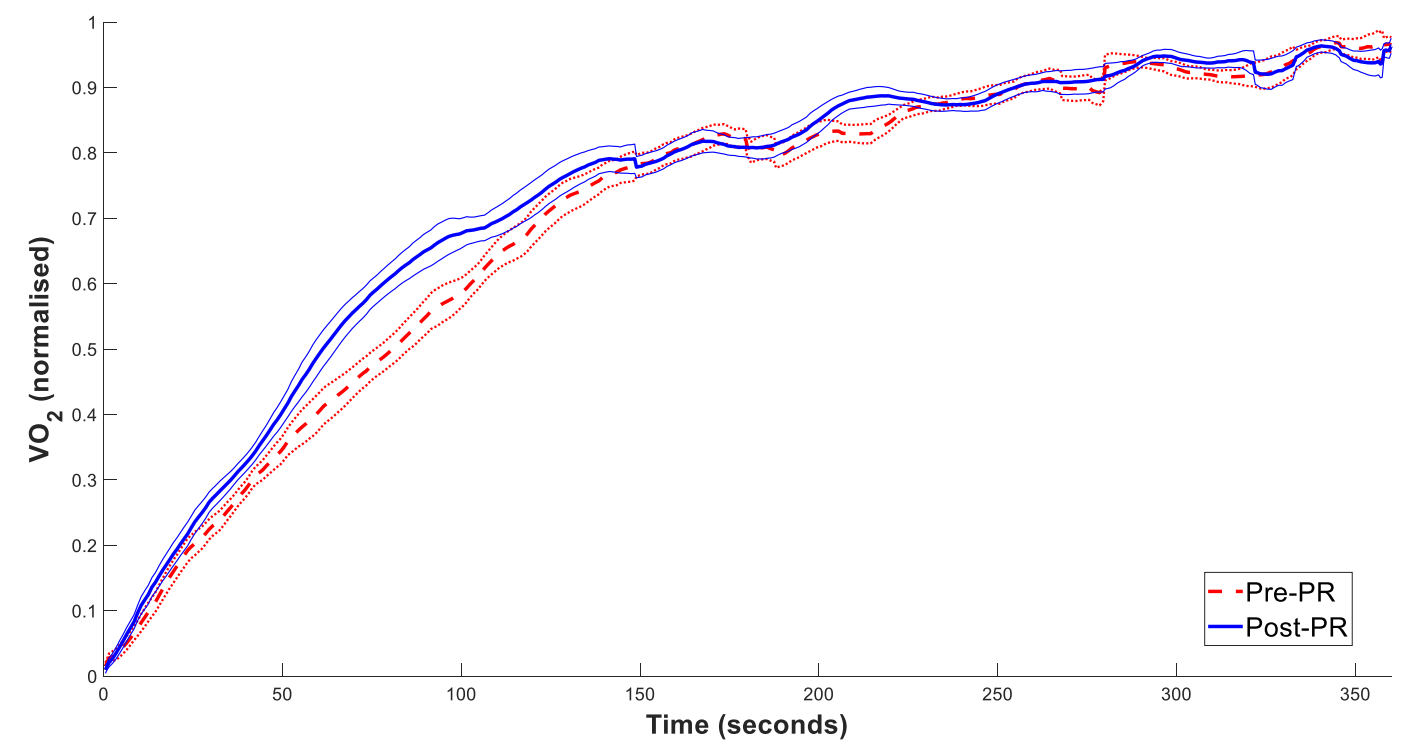

(b)

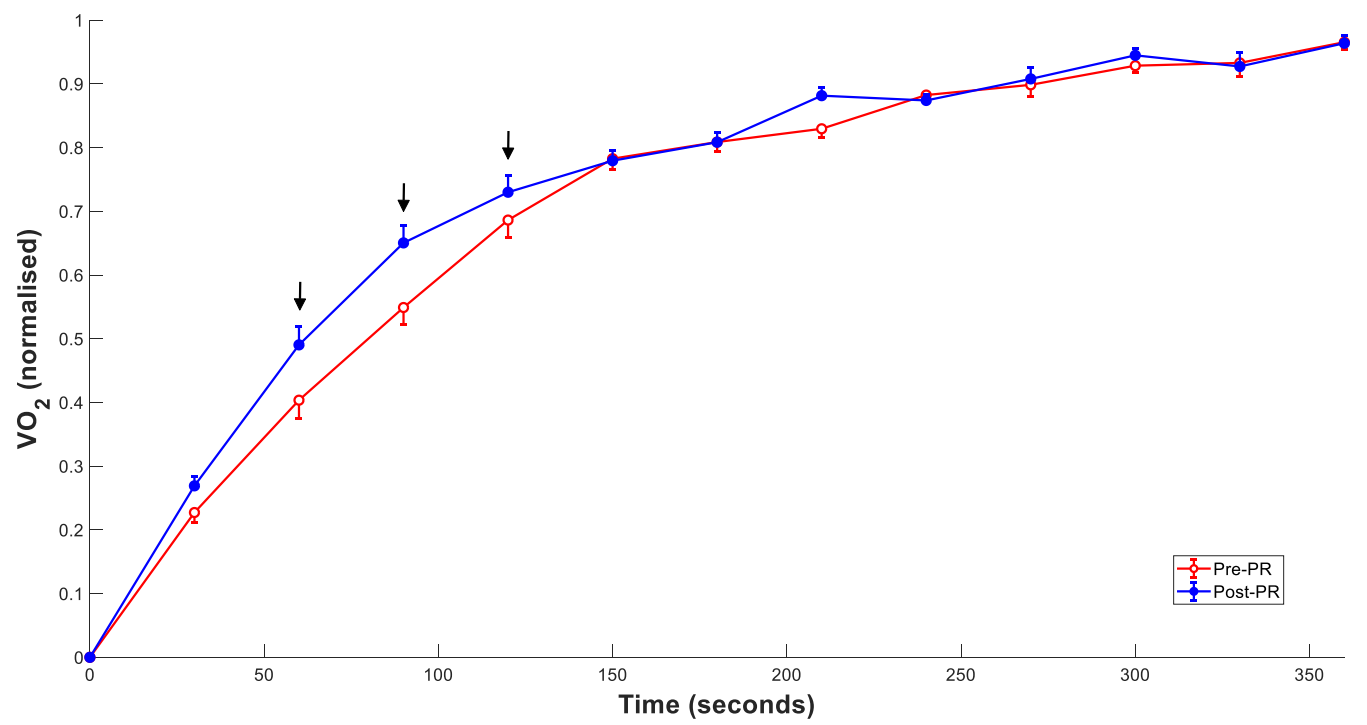

Figure 6.7 (a): Group-averaged normalised temporal $\mathrm{V}_{2}$ response to constant work rate exercise: prerehabilitation (red, dashed line) and post-rehabilitation (blue, solid thick line) and corresponding SEM values (pre: red, dotted: post: blue, solid thin); (b): $\dot{\mathrm{VO}}_{2}$ responses from (a) resampled at 30 second intervals; arrows = significant difference pre vs post $(p<0.05)$ 
(a)

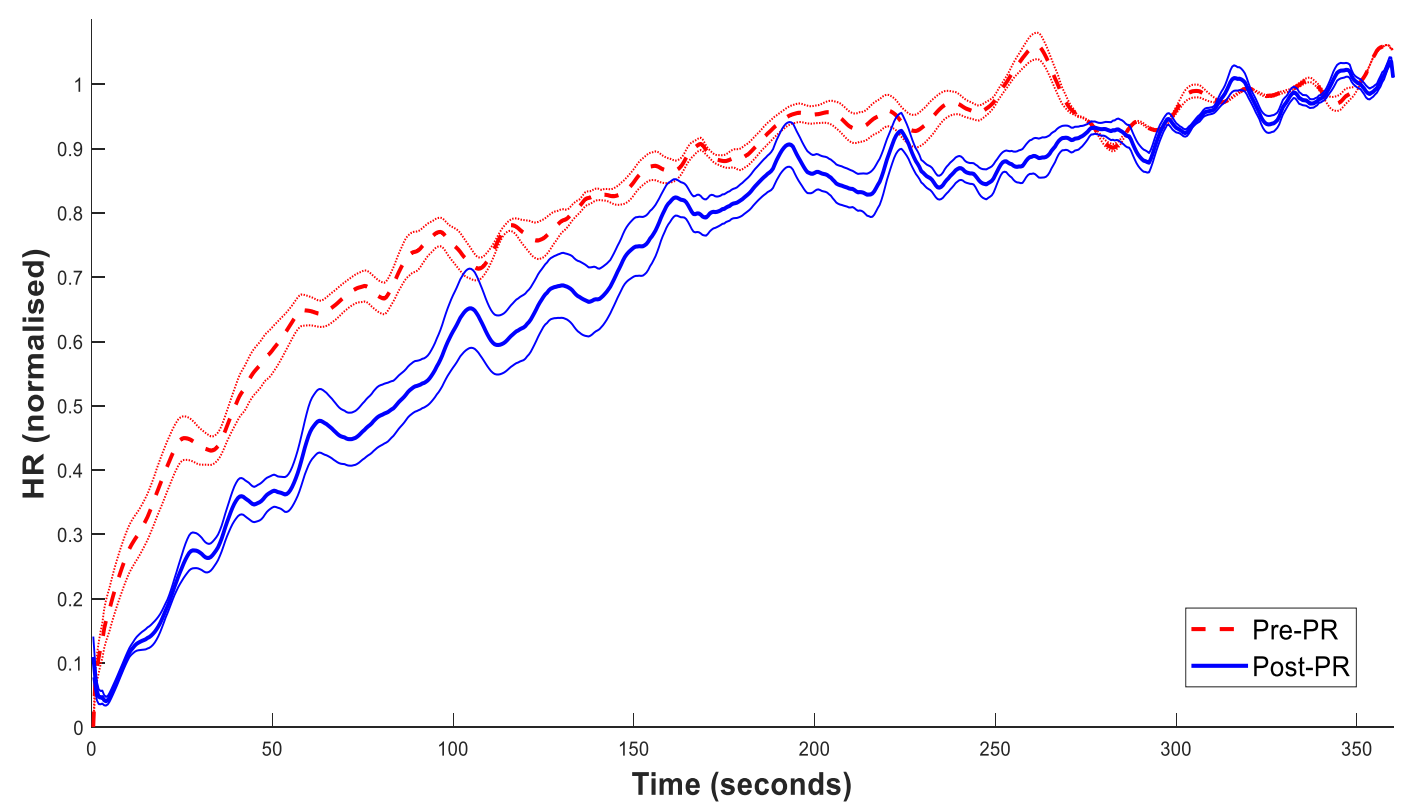

(b)

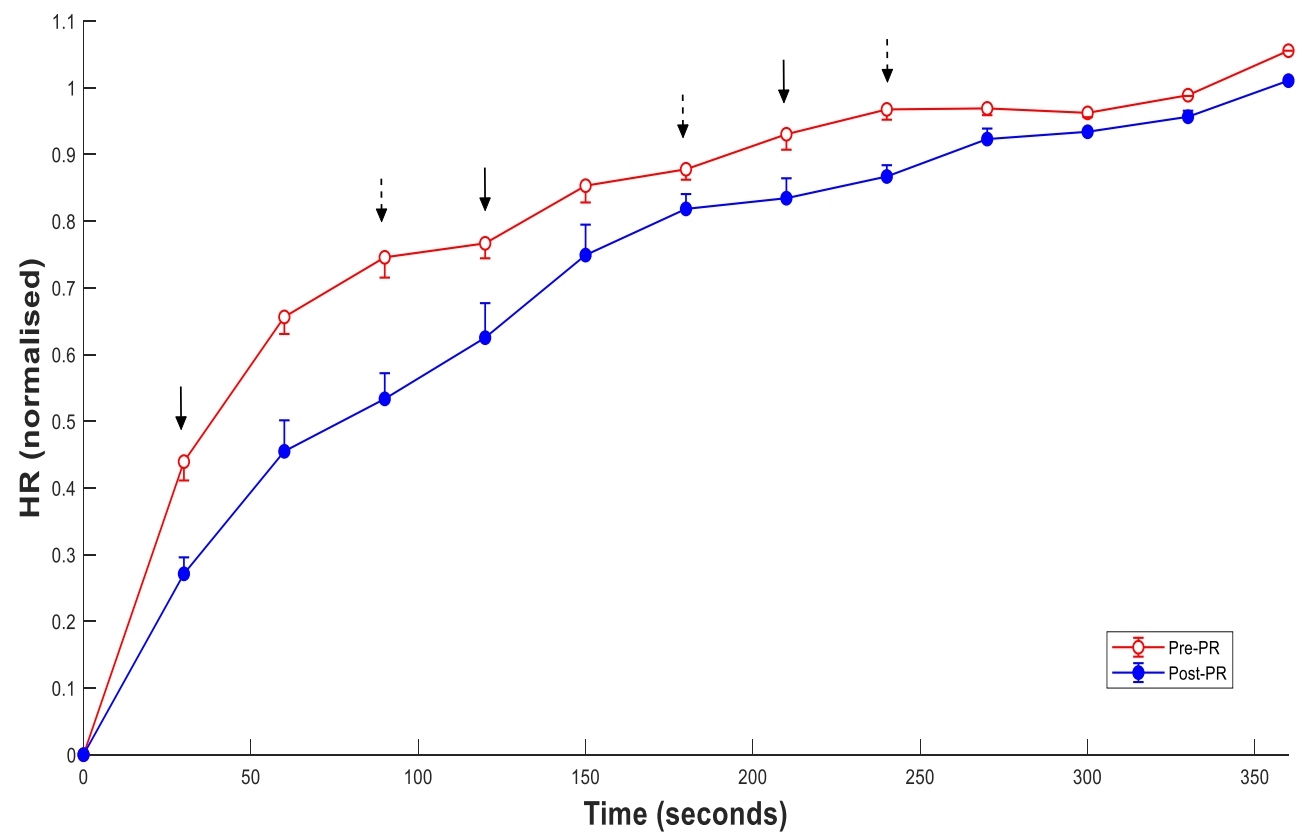

Figure 6.8 (a): Group-averaged normalised temporal HR response to constant work rate exercise: rerehabilitation (red, dashed line) and post-rehabilitation (blue, solid thick line) and corresponding SEM values (pre: red, dotted: post: blue, solid thin); (b): HR responses from (a) resampled at 30 second intervals; sold arrows $=$ significant difference pre vs post $(p<0.05) ;$ dashed arrows $=$ difference pre vs post $(p<0.10)$ 


\subsection{Discussion}

To better understand the influence of physical rehabilitation on cardiorespiratory function in IPF we quantified dynamic respiratory and cardiac responses to metabolic challenge. We found that following exercise-based PR IPF patients' rates of oxygen uptake improved significantly during the first two minutes following the onset of heavy-intensity exercise, and their heart rates were similarly reduced during (and beyond) this period.

We modelled the temporal oxygen uptake $\left(\mathrm{V}_{2}\right)$ response to heavy intensity exercise, characterising the response dynamics in terms of the mean response time (MRT). The MRT for $\dot{\mathrm{V}}_{2}$ in our participants was 108 seconds prior to rehabilitation, similar to the findings of McNarry et al. [16] who noted an MRT of 92 seconds (2SD range 73-111 seconds) in three IPF patients during heavy cycling exercise. This mean response time is substantially slower than that observed for similarly aged healthy individuals (McNarry reported an MRT of 52(12) seconds [16] whilst Breese et al. [150] reported an MRT of 66 seconds in healthy males). We also observed a $21 \%$ reduction in MRT to 85 seconds in patients who completed our PR programme. This demonstrates a substantially faster oxygen uptake response to metabolic challenge following exercise rehabilitation, which was notably most statistically significant within the initial two minutes of exercise.

Although the peak heart rates we observed during heavy-intensity exercise were similar before and after rehabilitation, the rate at which heart rate attained this peak value was almost twice as slow following rehabilitation (MRT = 60.5 vs. 115.5 seconds). McNarry et al. [16] previously recorded values in a similar range (2SD range $=49-125$ seconds) in three IPF patients; these values are substantially greater than those they reported for healthy individuals (2SD range $=12-60$ seconds). Furthermore, we noted sustained postrehabilitation reductions in heart rate within the first four minutes of the start of exercise.

Suggestions of the mechanisms by which exercise rehabilitation might have improved the oxygen uptake responses in this study are, of course, speculative. The candidate pathways likely to have a role in such adaptations are those influencing i) pulmonary mechanical function, ii) alveolar-capillary gas-exchange, and iii) systemic arterial-tissue oxygen exchange. It is also likely that direct adaptations in cardiovascular function could influence systemic oxygen delivery (and usage). Exercise-induced adaptations of any of these might be contributing factors to the improved oxygen uptake dynamics we observed during exercise. Previous studies with COPD patients suggested that oxygen delivery to muscles might be the limiting factor in $\mathrm{VO}_{2}$ kinetics [20] [150] [151] , this being attributed to impaired HR kinetics (a proxy for cardiac output and blood flow) [152]. Similarly, McNarry et al. [16] interpreted slower $\mathrm{HR}$ and $\mathrm{V}_{2}$ kinetics as an indication of central oxygen delivery limitation in both COPD and IPF patients. In the present study we observed a combination of improved (faster) 
oxygen uptake and diminished (slower) heart rate in response to exercise in patients who had completed exercise rehabilitation. We suggest that these alterations together suggest an improvement in cardiac pumping efficiency, which might reflect a myocardial training effect or the normalisation of intrathoracic pressures following rehabilitation. Notably, OUES (the ratio of oxygen uptake to minute ventilation) was unchanged by rehabilitation.

\subsection{Strengths and limitations of the study}

The current study extends our knowledge of the respiratory and cardiac responses to a structured PR programme. This was a two-hour programme which was conducted twice per week for a duration of eight weeks, each structured into two sessions. The first session was the exercise component and the second session focused on educating participants about IPF and living with the condition. This programme was conducted by a multidisciplinary team which included physiotherapists, physical trainers, occupational therapists, dieticians and specialist nurses. The study was conducted using a fairly heterogeneous but well-characterised group of IPF patients. During the recruitment process each patient's medical history was examined the exclusion criteria mentioned in Section 3.2.2 were strictly adhered to. Each participant fulfilled the 2011 American Thoracic Society and European Respiratory Society criteria [2] for diagnosis of IPF. Furthermore, these individuals had undertaken spirometry testing followed by high resolution computed tomography (HRCT) during the diagnosis process at an early stage by the specialist clinicians at Morriston Hospital, Swansea. HRCT scans indicating a predominant honeycomb pattern in the subpleural regions of the lung confirmed the presence of IPF. When this wasn't easily confirmed, surgical lung biopsy was conducted to confirm the presence of IPF. This study also took a novel approach to evaluating the oxygen uptake dynamic response in IPF patients (using MRT to analyse the data) since our empirical patient data showed no evidence of the 'fast' and 'slow' components that are present in the $\mathrm{VO}_{2}$ responses of healthy individuals. The study also had some limitations. Whilst we consider our sample of 15 patients to be representative of people with IPF, the relatively small number of study participants will have reduced the statistical power of our analyses. The present study used heart rate kinetics as a tool for estimating oxygen delivery to tissue; we did not directly assess muscle deoxygenation as a method of measuring muscle oxygen saturation. Furthermore, we did not used an age-matched control group since the purpose of the study was to look at the effects of rehabilitation on the same patients using identical methods pre- and post-testing. 


\subsection{Concluding comments}

As far as we are aware, this is the first study to assess physiological changes in cardiorespiratory function before and after a bespoke programme of PR, in people with UIP/IPF. We applied novel approaches to evaluate these changes and to provide a comprehensive profile of cardiorespiratory performance in UIP before and after PR. Specifically, PR improved the rate of oxygen uptake during heavy-intensity exercise, despite heart rates being substantially lower during this time, implying improvement in cardiac pumping efficiency. This suggests that PR might increase systemic arterial-tissue oxygen exchange and/or influence cardiovascular function to improve systemic oxygen delivery. Our findings suggest that individuals with UIP should find it easier to perform the activities of daily life, including those requiring substantial metabolic demands, after undertaking an appropriately-designed PR programme. 


\section{Chapter 7}

\section{Summary and concluding comments}

\subsection{Main outcomes}

\subsubsection{Patient perspectives}

It was evident that many IPF patients experience psychological trauma and depression following diagnosis. Patients indicated that the main causes of this were a lack of public awareness about the disease, poor or ambiguous information given to patients, and the unpredictable progression and prognosis of the disease. The absence (or inadequacy) of information about the disease is a major worry amongst patients, especially with regard to information on life expectancy, which makes them feel vulnerable. A key message on 'coping' that emerged from speaking to these patients was that acceptance of their condition led to a sense of optimism. Participants reported using appraisal-focused coping strategies to change their perspectives (thinking positively) and emotion-focused strategies to overcome depression (the main opportunity for emotional expression being an IPF support group). Family and support groups were also important mechanisms in helping patients to overcome depression and to change their lifestyles in order to help cope with the disease. The support group also facilitated problem-focused coping: individuals exchanged knowledge and experience and gave one another tips on how to live with their condition.

Based on these findings it is recommended that care should be taken when providing patients with information about the disease for the first time. Information should be more detailed, and it should be delivered to the patient through a medical professional who can emphasise that IPF is a subjective condition that affects individual patients differently. Patients and family members should also be guided towards counselling in order to better help with the management of trauma associated with the disease. Support groups should be added to the ongoing palliative care that these patients receive, and effective, evidence-based rehabilitation programmes should be made available to these patients.

\subsubsection{Physiological assessments}

This was the first study to assess the impact of a physical exercise rehabilitation programme on cardiorespiratory function in individuals with IPF. We quantified resting cardiac and respiratory system functions, and their dynamic responses to physical exercise across individuals' maximal exercise ranges. The 
study used novel approaches to functional evaluation, and it has provided the most comprehensive profile to date of cardiorespiratory performance adjustments in IPF.

Changes in HRV during the day 2 exercise protocol (gradually increasing workload to exhaustion tests) suggested that PR had a positive influence on patients' cardiac autonomic regulation profiles. Their resting cardiac autonomic states became more typical of healthy people, whilst enhanced parasympathetic influences during exercise suggested that they found the same exercise easier to perform after rehabilitation. There was also an indication (from acceleration and deceleration indices) that rehabilitation had improved the dynamic range of heart rate responsiveness in our patients. These changes suggest that physical rehabilitation had a positive 'normalising' influence on the cardiac autonomic modulation of heart rate in our patients. We speculate that this would be reflected as an improvement in patients' physical function during everyday tasks.

PR significantly improved the rate of oxygen uptake in response to the day 3 protocol (rapid-onset heavyintensity exercise), despite heart rates being substantially lower during this time. This suggests that our patients were achieving similar work outputs following PR but with a presumed improvement in cardiac pumping efficiency (i.e. meeting the required increase in cardiac output via stroke volume rather than heart rate changes). Individuals with IPF might therefore be expected to perform the stop-start activities of daily life (even those which represent substantial metabolic demands) more easily after undertaking an appropriatelydesigned exercise rehabilitation programme.

\subsection{Clinical relevance and impact}

The current study extends our knowledge of the respiratory and cardiac responses to supervised exercise rehabilitation in IPF patients. It should be borne in mind that IPF patients are acutely unwell and so the prospect of completing a physically- and physiologically-challenging study protocol would have been formidable. Therefore, although the number of recruited participants who completed the study was relatively small, this sample still allowed the compilation of a unique multivariate data set. Clinical colleagues verified that these participants were representative of individuals with IPF, and this provides confidence in generalising the results to the wider patient population. 


\subsection{Future perspectives and recommendations}

IPF is a relatively under-researched area compared with other chronic respiratory diseases (such as COPD). This was the first study to consider patients' cardio-respiratory responses to exercise at a physiological level. employing two very different exercise protocols that challenged their metabolic responses. This was also a highly novel study in that it explored the combined psychological, sociological and physical impacts of PR on IPF patients. Future work will hopefully use this study as a foundation to confirm the results presented here and to further develop our knowledge of this important clinical area.

It is highly recommended that future studies aim to recruit larger, heterogeneous groups of patients, enabling their categorisation according to age, gender, disease severity, medication regime and smoking status. Subgroup analyses based on these categories will yield further useful information regarding the relative efficacy of PR in the palliative care of IPF patients.

Future research will hopefully also employ additional methods for the accurate assessment of patients' cardiac output dynamics, their muscle oxygen saturation levels, and their muscle strength and fatigue levels. These studies should again include carefully-conducted interviews with participants (pre and post PR) to evaluate their personal opinions and experiences of PR: together with participant categorisation (as discussed above) this will facilitate a more detailed appreciation of the differential impacts of age, gender and disease severity on patients' perspectives regarding PR.

Finally, the IPF staging system presented in Appendix E could be used to record and interpret the impact of PR for individual patients, using our three-day assessment protocol. A description of this staging system and instructions on how to use it is given below and in Appendix $\mathrm{E}$.

\subsection{Staging and tracking system}

In general, a staging system aims to define a classification criterion that is practical, informs prognosis and impacts the choice of therapeutic modality. Staging systems are commonly used in healthcare for guiding medication and the management of a disease (for example, as used in cancer, HIV/AIDS and COPD [153] [154]). Staging in IPF is an important part of diagnosis to allow health professionals to guide treatment methods and counselling for patients [155] [156]. Some of the baseline parameters that could be useful in this regard includes FVC, DLco, 6MWT distance and dyspnoea score. Although not globally used, several groups have attempted to provide a basis for a more complex approach for staging systems to IPF [2]. Bois et al. [157] developed a point-based system that consisted of four predictors (age, recent respiratory hospitalization, baseline FVC and-24 week change in FVC), which gives a composite score that can accurately 
predict the 1-year risk of death [157]. Another system [158] used a multidimensional risk prediction model called the "GAP" comprising of four baseline variables (gender, age, FVC and DL $\mathrm{L}_{\mathrm{co}}$ ) and an associated scoring system knows as the GAP index, linked to the three traditionally-used disease stages (Stage 1, 2 and 3 or mile, moderate and severe) [158].

Extending the concept of a staging system, we suggest that a system for 'staging' and 'tracking' a patient's physiological responses to PR would be clinically useful. Such a system would enable evaluation of the benefit derived by individual patients who engage in a PR programme. PR can be considered as a palliative approach to easing the physical limitations experienced by these patients, thereby improving their QoL. On the presumption that post-rehabilitation benefits are likely to be influenced by disease severity, a 'PR outcome staging' system should provide indicative ranges of expected physiological improvement according to an individual's disease stage. We attempted to accomplish this by dividing our study population in to three different categories based on their FVC: 1) FVC > 80\% predicted (low disease severity), 2) FVC of 55-80\% predicted (moderate disease severity) 3) FVC $<55 \%$ predicted (high disease severity). Next, we separated male and female participants in each group and averaged each of the physiological variables which we selected for inclusion in the staging system (Table E.1). The physiological variables included were chosen to make the system practical to use in a clinical setting by health professionals (variables requiring a more complicated or secondary calculation, such as HRV, were excluded).

Figure E.1 shows the format of a possible patient record that uses the proposed rehabilitation staging and tracking system:

- The red, yellow and green coloured columns represent low, moderate and high disease severity respectively.

- Patients are required to undergo standard spirometry testing to measure their FVC before PR. Depending on the FVC result they would be categorised into one of the three coloured columns (FVC above 80, FVC between $80-55$, FVC below 55 ). The physiological variables in the respective columns are the disease severity-specific values expected for the individual.

- Next the patient would undertake an incremental bicycle test with an increase of 10-15 Watts per minute, and the results would be reported in the pre-rehabilitation column.

- Once the PR is completed by the patient, the same procedure would be followed and the results would be recorded in the post-rehabilitation column (new FVC value).

- Next, the change in the physiological values (pre vs post) would be calculated and would be reported in the \% change column. 
As the final step, post-PR values would be compared with the reference values, and the outcome of the rehabilitation programme on the patient could then be assessed

7.5 Knowledge transfer IPF is a relatively unrecognised disease in Sri Lanka (SL). One of the future possible extensions of this study is to conduct collaborative work between Swansea University and General Sir John Kotelawala Defence University in SL (the author's home university). Some of the possibilities of such collaboration include working with the Collage of Respiratory Physicians (SL) to improve the knowledge base about the disease in SL and to encourage medical professionals in the curative sector to introduce PR as a method of treatment for the disease. Another possibility is to work with the Ministry of Health (SL) to develop policies and awareness campaigns to identify and treat IPF patients found among the general population in SL. The knowledge gained from the studies reported in this thesis would be of great importance (and directly transferable) to SL: it would help in establishing physiological assessment laboratories (both in the healthcare and university sectors) to train future researchers about UIP/IPF, whilst the newly-established qualitative understanding of UK patients' perspectives would be invaluable in establishing patient-centred support systems. 


\section{Appendix A: Ethical Approvals}

Applied Sports Technology Exercise and Medicine Research Centre (A-STEM)

\section{APPLICATION FOR ETHICAL COMMITTEE APPROVAL OF A RESEARCH PROJECT}

In accordance with A-STEM and College of Engineering Safety Policy, all research undertaken by staff or students linked with A-STEM must be approved by the A-STEM Ethical Committee.

\section{RESEARCH MAY ONLY COMMENCE ONCE ETHICAL APPROVAL HAS BEEN OBTAINED}

The researcher(s) should complete the form in consultation with the project supervisor. After completing and signing the form students should ask their supervisor to sign it. The form should be submitted electronically to Prof Mike McNamee ( and Dr Melitta McNarry

Applicants will be informed of the Committee's decision via email to the project leader/supervisor.

\section{TITLE OF PROJECT}

Investigating the perceptions and opinions of patients with IPF regarding pulmonary rehabilitation programmes

\section{DATE OF PROJECT COMMENCEMENT AND PROPOSED DURATION OF THE STUDY}

$26 / 04 / 2016$ to $26 / 4 / 2018$

\section{NAMES AND STATUS OF RESEARCH TEAM}

State the names of all members of the research group including the supervisor(s). State the current status of the student(s) in the group i.e. Undergraduate, Postgraduate, Staff or Other (please specify).

Prof. M.J. Lewis (Staff)

Dr. M. Mcnarry (Staff)

Dr. J. Hudson (Staff)

Mr. S.P. Senanayake (PG Student) 


\section{RATIONALE AND REFERENCES}

With reference to appropriate sources of information (using the Harvard system), describe in no more than 200 words the background to the proposed project.

The two major classes of chronic respiratory disease are chronic obstructive pulmonary disease (COPD) and interstitial lung disease (ILD). COPD is characterised by obstructed air flow in the airways, whilst ILD is characterised by the build-up of scar tissue (fibrosis) in the lungs. Patients with ILD are less able to transfer oxygen from the air they breathe into the blood vessels within the lungs, making them feel breathless during simple everyday activities like walking. COPD patients also become breathless during activity but unlike the situation in ILD this is caused by the obstructed airflow.

Treatment options consequently differ for COPD and ILD patients: COPD patients typically take medication to relieve the airflow restriction whilst ILD patients typically require oxygen therapy. Pulmonary rehabilitation (PR) programmes are also used to help alleviate symptoms in both COPD and ILD. PR is well-established in the management of patients with COPD and is effective in improving exercise performance (Spiruit et al., 2013). However, the benefit of PR for ILD patients is less clear (Dowman, 2014). According to Spiruit et al. (2013), pulmonary rehabilitation has little effect on ILD and the small amount of improvement that is achieved is not sustained beyond six months. A possible reason for this discrepancy between COPD and ILD responses to $P R$ is that rehabilitation programmes have typically been designed to address the needs of COPD patients.

Abertawe Bro Morgannwg University (ABMU) Health Board runs a series of PR programmes lasting eight weeks. Patients who have been referred for PR, together with associated healthcare professionals, are also invited to participate in a monthly 'PR support group'. The group provides an informal setting within which patients and professionals can discuss issues relating to their condition and PR participation.

The overall aim of this study is to seek opinion and comments on PR from the members of a monthly PR support group. This will include (1) patients with either COPD or the most common form of ILD, idiopathic pulmonary fibrosis (IPF) and (2) the health professionals who are responsible for running the PR programme. Some of the patients will already be participating in PR and others will be awaiting enrolment into a future PR programme.

References

1. Dowman, L., Hill, C. J., \& Holland, A. E. (2014). Pulmonary rehabilitation for interstitial lung disease. Cochrane Database Syst Rev, 10.

2. Spruit, M. A., Singh, S. J., Garvey, C., ZuWallack, R., Nici, L., Rochester, C., ... \& Pitta, F. (2013). An official American Thoracic Society/European Respiratory Society statement: key concepts and advances in pulmonary rehabilitation. American Journal of Respiratory and Critical Care Medicine 188(8), e13-e64

\section{OBJECTIVES}

State the objectives of the project, i.e. one or more precise statements of what the project is designed to achieve. 
The objective of the present study is to ascertain patients' and physiotherapists' opinions and perceptions of PR. Specifically, we wish to investigate patients' expectations of PR prior to undertaking it, what their feelings, opinions and perceptions are during the 8-week PR program and, following its completion, what their reflections are on the program. We also wish to obtain insight from the physiotherapists' perspective with regard to its apparent effectiveness, practicalities and areas they would like to change in the future.

\subsection{STUDY DESIGN}

- outline the chosen study design (e.g. cross-section, longitudinal, intervention, RCT, questionnaire etc)

Qualitative study utilizing focus groups

\subsection{STUDY DESIGN}

- state the number and characteristics of study participants

- state the inclusion criteria for participants

- state the exclusion criteria for participants and identify any requirements for health screening

- state whether the study will involve vulnerable populations (i.e. young, elderly, clinical etc.)

- state the requirements/commitments expected of the participants (e.g. time, exertion level etc)

- Approximately 20 individuals (either patients with COPD or IPF, or healthcare professionals) will be invited to participate

- Participants will be patients who are attending the ABMU PR support group held at Penuel Chapel, Loughor and the healthcare professionals (physiotherapists) involved in their treatment.

- Inclusion criteria - All participants must (1) have been diagnosed with either IPF or COPD, (2) be aged 18 years or older and (3) be able to write and converse in English (to facilitate data collection).

- Exclusion criteria - Inability or unwillingness to provide informed consent to participate in the study.

- Participation in these focus groups is independent of patient care/treatment and will not influence their selection for participation in PR by the physiotherapists at Morriston or Singleton hospitals.

Study Design

The participants will be invited to attend a small focus group session in groups of 4-5 participants. These sessions will be arranged at a time and location convenient for the participants; the location will be a quiet but public area and two members of the research team will be present at all times. The groups will be chosen according to those who are waiting to take part in PR, those who have already completed PR and those who do not wish to take part in PR. Each focus group will take approximately 45-60 minutes and will be conducted according to the Chatham house rules.

- Participants will be free to withdraw from the study at any time they wish

- To maintain anonymity, pseudonyms will be given to individual participants before commencement of the audio and video recording

- Participants will be asked to wear a badge showing their 'identity' and to state this identity before speaking

- If a participant wishes to leave the study at any point, their comments will be removed from the transcripts and from the audio and video recordings. 


\subsection{PARTICIPANT RECRUITMENT}

How and from where will participants be recruited?

A monthly support group is held for patients with IPF at Penuel Baptist Church, Glebe Road, Loughor, Swansea. We will initially attend one of these monthly meetings and present an overview of the study. Attendees who will be invited to participate in the study. Permission will be sought from the chairman of the support group (Mr. David Rees) to attend this meeting. Those interested in participating will be provided with an information sheet and consent form and given a week to decide if they wish to take part in the study. If a patient wishes to take part in the study after this time, they may still do so by contacting one of the members of the research team (provided in the participant information sheet). It will be made clear to the potential participants that participation is entirely voluntary, that they are free to withdraw at any point and that participation will not influence their selection for PR.

\subsection{DATA COLLECTION METHODS}

- describe all of the data collection/experimental procedures to be undertaken

- state any dietary supplementation that will be given to participants and provide full details in Section 6.5

- state the inclusion of participant information and consent forms (in appendices)

- refer to the use of the ACA/ACSM health screening questionnaire where appropriate (usually for maximal effort exercise)

Data collection will involve interviewing participants in focus groups with patients with IPF. An example of the semi-structured interview guide is provided in Appendix 1 which follows the steps outlined by Rubin and Rubin (2012), whereby the interviews start with introductory questions, followed by transition questions, then the main questions and finally summary questions. Krueger and Casey (2000) have defined focus groups as "carefully planned series of discussions designed to obtain perceptions on a defined area of interest in a permissive, non-threatening environment". We will use their guidelines to facilitate the focus group discussions. An exact list of questions to be asked in this study cannot be provided as the questions will be led by the participants' responses. Interviews will last approximately 45-60 minutes and will be audio and video recorded to assist with transcription at a later date. All participants will be fully debriefed at subsequent IPF support group meetings.

- If a participant indicates that they do not wish to be video recorded, only audio recordings will be made

- If a participant does not wish to be audio recorded, they will be given an explanation of the importance of the recording and what the research team will do with the data along with how we will store and eventually dispose of this.

- If participants still do not consent to interview recording, they will be excluded from the study.

- We will not define a minimum number of participants required to conduct each interview (i.e. no 'quorum'). In the event of a participant 'dropping out' of a group, the interviews will still continue as planned. 
All focus groups will be conducted by two named members of the research team. One of the two members is Mr. SP Senanayake (PhD student) and the other member would be either Prof. M.J. Lewis, Dr. M. McNarry or Dr. J. Hudson. The senior member of the team will be present along with the inexperienced student to ensure that a high and consistent standard is maintained throughout the interviews.

\subsection{DATA ANALYSIS TECHNIQUES}

- describe the techniques that will be used to analyse the data

Qualitative analysis of the data from the focus groups will use a thematic approach (see Braun and Clarke, 2006) to illustrate common themes pertaining to an under researched area. Using this approach, notes and initial impressions are taken by the researcher during the focus groups. These ideas are then tracked and continually revised throughout transcription, and later themes are developed based on underlying meaning. Furthermore, all focus groups will be transcribed verbatim and pen-profiles will be produced to provide a quantitative analysis of the key themes raised.

\subsection{STORAGE AND DISPOSAL OF DATA AND SAMPLES}

- describe the procedures to be undertaken for the storage and disposal of data and samples

- identify the people who will have the responsibility for the storage and disposal of data and samples

- Identify the people who will have access to the data and samples

- state the period for which the data will be retained on study completion (normally 5 years, or end of award)

- The recordings and verbatim transcripts will be kept on password-protected hard drives.

- Video recordings and verbatim transcripts will be stored in different computers.

- The hard copies of the participants' information will be stored separately under lock and key within Swansea University premises.

- All the electronic copies of data from the study will be encrypted using appropriate software.

- All participant names will be removed and replaced with pseudonyms to ensure confidentiality.

- The data will be accessible only by the research team and will be retained for seven years following the student's academic award.

- Prof M.J. Lewis will be responsible for deletion of all copies of material following this point. All recordings will be deleted from recording devices immediately on transfer to a password-protected hard drive and on the same day as the interviews.

\subsection{HOW DO YOU PROPOSE TO ENSURE PARTICIPANT CONFIDENTIALITY AND ANONYMITY?}

If patients mention their names or those of others during focus groups their names will be replaced with pseudonyms during transcription.

Given the public nature of focus groups it is not possible to guarantee anonymity of participants. However, we will seek to ensure confidentiality by introducing Chatham House Rules at the outset of the focus group, asking participants to indicate that they will not share information outside of the room. If participants are not comfortable taking part in a focus group, we will provide them with an opportunity to complete an individual interview to further protect their confidentiality. 
All the data generated from this study will be treated in the strictest confidence and patient anonymity will be maintained throughout. All patient data will be anonymized with regard to any future publications relating to this study and data will be accessible only by the research team. Data will be stored in University computers (including the student's work computer), which are password protected.

6.8 PLEASE PROVIDE DETAILS OF ANY DIETARY SUPPLEMENTATION (DELETE IF NOT APPLICABLE)

Not applicable

\section{LOCATION OF THE PREMISES WHERE THE RESEARCH WILL BE CONDUCTED.}

- list the location(s) where the data collection and analysis will be carried out

- identify the person who will be present to supervise the research at that location

- If a first aider is relevant, please specify the first aider

Penuel Baptist Church, Glebe Road, Loughor, SA4 6QS or at a convenient public location for all participants in the focus group.

The interviews will be conducted by Mr. SP Senanayake in the presence of either Prof. M.J Lewis, Dr. M. McNarry or Dr. J. Hudson.

\section{POTENTIAL PARTICIPANT RISKS AND DISCOMFORTS}

- identify any potential physical risk or discomfort that participants might experience as a result of participation in the study.

- identify any potential psychological risk or discomfort that participants might experience as a result of participation in the study.

- Identify the referral process/care pathway if any untoward events occur

In the focus groups, participants may potentially experience discomfort sharing personal experiences, but this will be minimized by the above confidentiality measures and by not coercing people to say anything they don't want to. This will be reinforced at the start of each focus group. If they feel any sort of discomfort during any stage of the study, they can withdraw from the study without any explanation anytime they wish. If they wish to talk to someone about their experiences, they can talk to their consultant (Dr Kim Harrison), the physiotherapist (Angharad Ladd) or the chair of the REC (Prof M.J. McNamee). The procedures to be followed in the event of participant distress during Interviews/Focus Groups are described in Appendix 2.

\subsection{HOW WILL INFORMED CONSENT BE SOUGHT?}

Will any organisations be used to access the sample population?

Will parental/coach/teacher consent be required? If so, please specify which and how this will be obtained and recorded? 
An initial introduction to the study will be given by the chairman of the group, followed by a summary of the study by Dr Melitta McNarry. Participants will be invited to take part and those who express an interest with be provided with an information sheet. Written informed consent of the participants will be obtained by the research team prior to data collection.

\subsection{INFORMATION SHEETS AND CONSENT/ASSENT FORMS}

- Have you included a Participant Information Sheet for the participants of the study? YES

- Have you included a Parental/Guardian Information Sheet for the parents/guardians of the study? N/A

- Have you included a Participant Consent (or Assent) Form for the participants of the study? YES

- Have you included a Parental/guardian Consent Form for the participants of the study? N/A

10. IF YOUR PROPOSED RESEARCH IS WITH VULNERABLE POPULATIONS (E.G. CHILDREN, PEOPLE WITH A DISABILITY), HAS AN UP-TO-DATE DISCLOSURE AND BARRING SERVICE (DBS) CEHCK (PREVIOUSLY CRB) IF UK, OR EQUIVALENT NON-UK, CLEARANCE BEEN REQUESTED AND/OR OBTAINED FOR ALL RESEARCHERS? EVIDENCE OF THIS WILL BE REQUIRED.

Not applicable

\section{STUDENT DECLARATION}

Please read the following declarations carefully and provide details below of any ways in which your project deviates from these. Having done this, each student listed in section 2 is required to sign where indicated.

- "I have ensured that there will be no active deception of participants.

- I have ensured that no data will be personally identifiable.

- I have ensured that no participant should suffer any undue physical or psychological discomfort (unless specified and justified in methodology).

- I certify that there will be no administration of potentially harmful drugs, medicines or foodstuffs.

- I will obtain written permission from an appropriate authority before recruiting members of any outside institution as participants.

- I certify that the participants will not experience any potentially unpleasant stimulation or deprivation.

- I certify that any ethical considerations raised by this proposal have been discussed in detail with my supervisor.

- I certify that the above statements are true with the following exception(s):"

Student/Researcher signature:

Date: 08.04.2016

12. SUPERVISOR'S APPROVAL

Supervisor's signature:

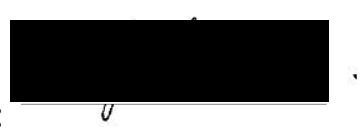

Date: $22 / 05 / 16$ 


\section{PARTICIPANT INFORMATION SHEET}

(Date: 03/05/2016)

Project Title: Patients prospective in pulmonary rehabilitation

Contact Details: Sameera Peumal Senanayake -

\section{Invitation Paragraph}

I would like to invite you to take part in our research study. My name is Sameera Senanayake and I'm a firstyear PhD student in the A-STEM (Applied Sport Technology Exercise and Medicine) Research Group at Swansea University. In order to help you decide whether you would like to participate in the study, it is important to understand why the research is being done and what it will involve for you. Please take your time to read the following information carefully and discuss it with someone else if you wish. If there is anything you do not understand or about which you would like more information, please do not hesitate to ask for further information.

\section{What is the purpose of the study?}

To talk to patients with IPF about their thoughts regarding pulmonary rehabilitation programmes. We would like to talk to you regardless of whether you have already completed the programme, are waiting to or do not want to complete it. The information obtained from the interviews will be used to help design new rehabilitation programmes that are more effective for IPF patients. 


\section{Why have I been chosen?}

You have been chosen because you have been diagnosed with IPF and you have already completed, are waiting to complete or do not wish to complete a pulmonary rehabilitation programme.

\section{What will happen to me if I take part?}

If you are happy to take part, you will be invited to participate in a focus group session with 3-4 other people with IPF. During this session, we will discuss your opinions and thoughts about pulmonary rehabilitation. This session will be audio and video recorded but there are no right or wrong answers, we are just interested in what your thoughts and opinions are.

\section{What are the possible disadvantages of taking part?}

At first, you might feel a little shy talking about your feelings and thoughts about pulmonary rehabilitation and IPF. However, we will do our best to put you at ease - everyone is likely to be feeling the same way. If at any point you would like to stop taking part, you are free to withdraw from the study with no fear of penalty. Furthermore, you might potentially experience discomfort sharing personal experiences within the focus groups. We will make every effort to avoid this - for example we will not try to make you talk about anything that you don't want to. If you do feel any sort of discomfort during any stage of the study, you can withdraw from the study without any explanation if you wish.

\section{What are the possible benefits of taking part?}

Your opinion and feedback will provide us with important information that we will use to help shape future rehabilitation programmes for those with IPF.

\section{Will my taking part in the study be kept confidential?}

All the data gathered will be treated in the strict confidence and patient anonymity will be maintained throughout the study. If names are mentioned during the focus groups, these will be replaced with pseudonyms during transcription to ensure anonymity and confidentiality. Given the public nature of these 
discussions it is not possible to absolutely guarantee anonymity of participants among the focus group. However, we will seek to ensure confidentiality by asking you to indicate that you will not share information outside of the group. If you are not comfortable taking part in a focus group, we will provide you with an opportunity to complete an individual interview instead.

\section{What if I have any questions?}

If you have any further questions, we encourage you to contact the research team to discuss them. If you have any concerns regarding this study at any point, please let us know or talk to your consultant (Dr. Kim Harrison) or the chair of the research ethics committee who approved this study (Prof Mike McNamee, 


\section{PARTICIPANT CONSENT FORM}

(Version 1.1, Date: 3/05/2016)

\section{Project Title:}

Patient feedback and opinions on a programme of pulmonary rehabilitation

\section{Contact Details:}

Sameera Peumal Senanayake -

Prof. M.J. Lewis -

\section{Please initial box}

1. I confirm that I have read and understood the information sheet dated 03/05/2016 (version number 1.1) for the above

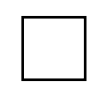
study and have had the opportunity to ask questions.

2. I understand that my participation is voluntary and that I am free to withdraw at any time, without giving any reason, without my medical care or legal rights being affected.

3. I understand that sections of any of data obtained may be looked at by responsible individuals from the Swansea University or from regulatory authorities where it is relevant to my taking part in research. I give permission for these individuals to have access to these records.

4. I agree to take part in the above study.

\section{Name of Participant}

Name of Person taking consent

Researcher

\section{Date \\ Signature}

Date

Signature

Signature 


\section{PARTICIPANT DISTRESS}

\section{Procedures to follow in the event of participant distress during Interviews/Focus Groups}

\section{Prior to the interview:}

Prior to conducting interviews, pilot interviews will be conducted in liaison with the supervisor. These interviews will provide the researcher with an opportunity to identify any questions that might lead to distress and where appropriate, take steps to rephrase or change these questions.

Before conducting the first formal interview, the student will meet with their supervisor to discuss to procedures that are in place in case a participant becomes distressed during an interview. The supervisor will also ensure the student feels prepared for the interview. The supervisor must be satisfied that the researcher is competent in conducting interviews before giving approval for the commencement of data collection.

Students will inform their supervisor where and when they are completing all interviews and in turn the supervisor will ensure the student has a means of contacting them when they are conducting interviews.

\section{During the interview:}

At the beginning of the interview the student will remind the participant that they can stop the interview at any time, that they can choose not to answer questions, and that there are no right or wrong answers to questions (so there is no fear of 'saying the wrong thing').

Once the interview begins, the researcher will be required to be aware of any potential indications of distress (e.g., withdrawing, visible upset, declining to answer numerous questions, shifting in seat, looking away from the interviewer, asking for the interview to end) and should air on the side of caution in all instances. If there is even the slightest indication that participants might be distressed students must immediately follow the procedure below:

1) The recording will be immediately stopped, and the participant will be asked if they are ok. At this point the participant will be asked if they want to take a break/end the interview/continue talking the participant's decision will be final. If the participant decides to take a break and continue with the interview, confirmation will be sought that the participant is actually comfortable continuing, and they will be reminded there is no penalty for withdrawing.

2) If the participant wishes to continue but remains distressed, the interviewer will make the decision to draw the interview to an end. At this point, the interviewer will commit to providing the participant with an opportunity to talk and ensure the participant is not visibly distressed when leaving the 
interview.

3) If the participant remains distressed and the researcher does not feel capable of managing the situation, they will contact their respective supervisor who will be available at all times during interviews by phone contact. Depending on the situation, the supervisor will either provide guidance to the student, speak directly to the participant over the phone, or make attempts to go and meet with the researcher and the participant.

4) If the participant has become distressed at any point in the interview, the student will ensure the participant has the contact details of the rest of the research team and remind them that they are free to contact any member of the research team if there is anything further, they would like to discuss.

5) The interviewer will also offer to provide the participants with a list of local contacts (e.g., counselling services, sport psychology services) if they would like them.

6) Following the interview, the student will debrief the interview with their supervisor and (if necessary) other senior members of the research team. A written record of the incident and the procedures followed will be made. 
First of all, let me thank you for taking part in this focus group discussion and agreeing to take part in this study.

1. If you think back to your life before being diagnosed with your medical condition, what are the things that you did before but are prevented from doing now?

2. What does have IPF mean to you?

3. How does IPF make you feel on a day-to-day basis?

4. In your own words, could you explain what you think pulmonary rehabilitation is?

a. What do you think is involved on a daily/weekly basis?

5. What do you think the possible effects of this type of pregame might be? These can be positive or negative!
a. Physically?
b. Mentally?
c. Socially?

6. What are your thoughts about participating in PR?
a. What are you looking forward to?
b. What are you worried about?
c. PR normally lasts 8 weeks, what do you think about this duration?

7. What do your family/friends think about the PR programme and participating in it?

8. Have you or do you know anyone who has undergone PR?
a. What did you/they think of it?
b. Would you/they do it again?
c. What would you/they change?

9. Away from PR, what do you do on a daily basis? What is your average week like?
a. What sort of physical activities do you enjoy/dislike?
b. What is your perception of exercise?
c. What would you change about your daily routine?

10. PR generally includes an educational component, what do you think it would be useful for this to include?

a. Who do you think the best people would be to attend this element? 


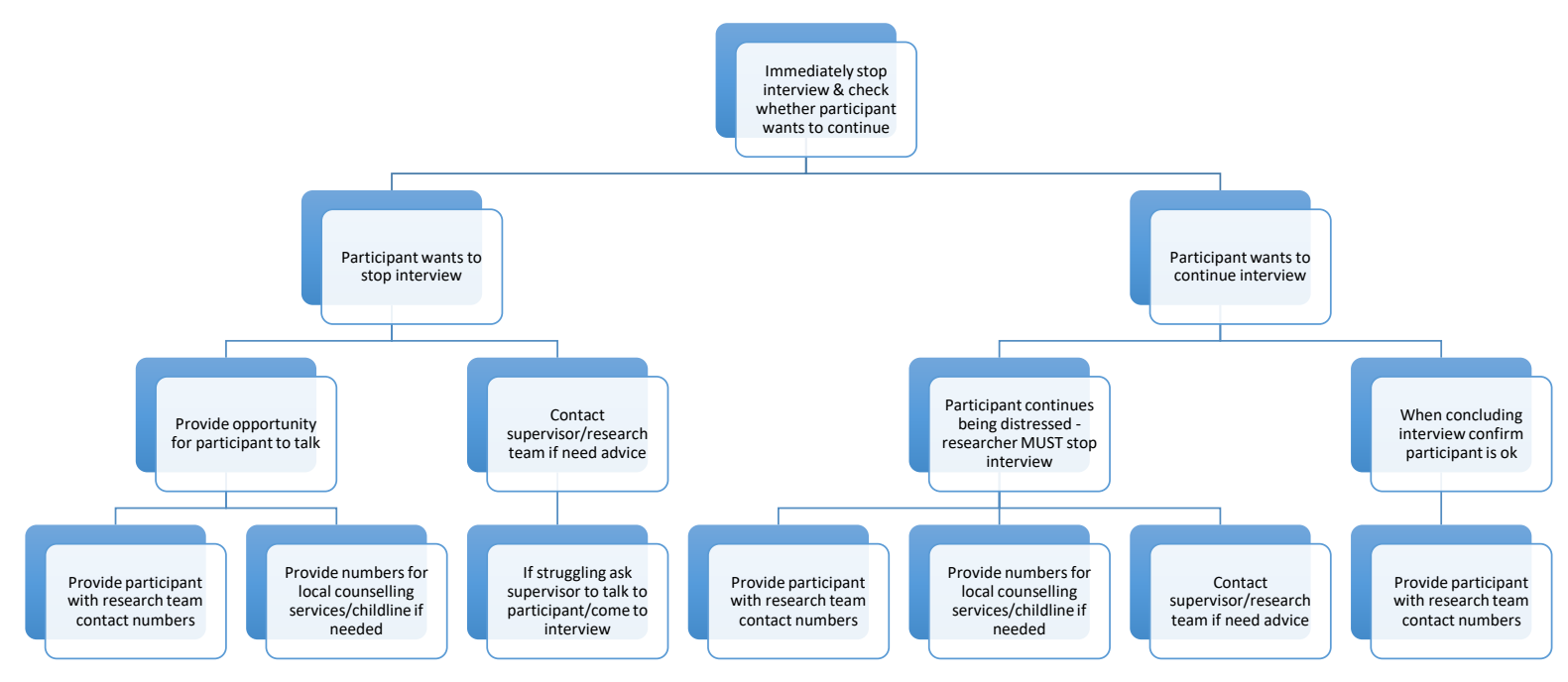

If participant has become distressed at any point you must debrief with supervisor and write up the steps that were taken throughout to manage the situation. 
Ymchwil lechyd a Gofal Cymrü

Health and Care

Research Wales
Gwasanaeth Moeseg Ymchwil Research Ethics Service

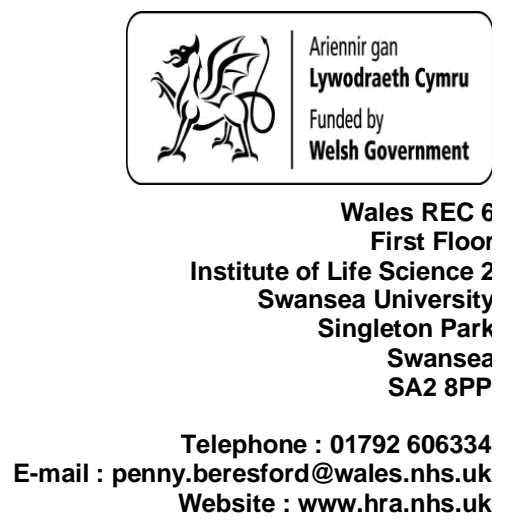

03 November 2016

Professor Michael J Lewis

College of Engineering

Swansea University, Bay Campus

Crymlyn Burrows

SA1 8EP

Dear Professor Lewis

Study title:

REC reference:

Amendment number:

Amendment date:

IRAS project ID:
Physiological and physical changes in patients with Idiopathic Pulmonary Fibrosis (IPF) following an eight-week pulmonary rehabilitation program

16/WA/0272

AM01

12 October 2016

206031

The above amendment was reviewed 02 November 2016 by the Sub-Committee in correspondence.

The members of the Committee taking part in the review gave a favourable ethical opinion of the amendment on the basis described in the notice of amendment form and supporting documentation.

\section{Approved documents}

The documents reviewed and approved at the meeting were:

\begin{tabular}{|l|l|l|}
\hline Document & Version & Date \\
\hline $\begin{array}{l}\text { Notice of Substantial Amendment (non-CTIMP) [inclusion of a } \\
\text { physical activity monitor] }\end{array}$ & AM01 & 12 October 2016 \\
\hline Participant information sheet (PIS) & 1.3 & 12 October 2016 \\
\hline $\begin{array}{l}\text { Research protocol or project proposal [exercise protocol flow } \\
\text { chart] }\end{array}$ & 1.3 & 12 October 2016 \\
\hline
\end{tabular}

Membership of the Committee

The members of the Committee who took part in the review are listed on the attached sheet. 
Ymchwil lechyd a Gofal Cymru

Health and Care

Research Wales
Gwasanaeth Moeseg Ymchwil Research Ethics Service

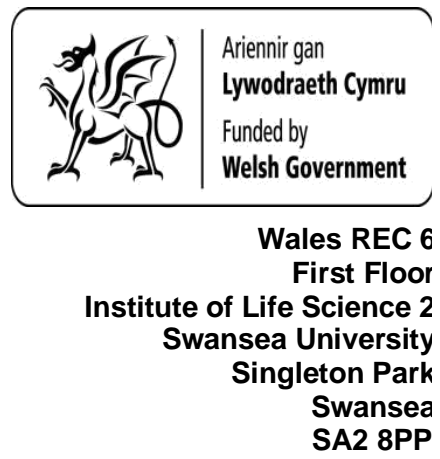

Telephone : 01792606334

E-mail : penny.beresford@wales.nhs.uk Website : www.hra.nhs.uk

11 October 2016

Professor Michael J Lewis

College of Engineering

Swansea University, Bay Campus

Crymlyn Burrows

SA1 8EP

\section{Dear Professor Lewis}

\section{Study title: \\ REC reference: \\ IRAS project ID: \\ Physiological and physical changes in patients with Idiopathic Pulmonary Fibrosis (IPF) following an eight-week pulmonary rehabilitation program \\ 16/WA/0272 \\ 206031}

Thank you for your email of 11/10/2016, responding to the Committee's request for further information on the above research and submitting revised documentation.

The further information has been considered on behalf of the Committee by the Chairman. We plan to publish your research summary wording for the above study on the HRA website, together with your contact details. Publication will be no earlier than three months from the date of this opinion letter. Should you wish to provide a substitute contact point, require further information, or wish to make a request to postpone publication, please contact the REC Manager, Ms Penny Beresford, penny.beresford@wales.nhs.uk.

\section{Confirmation of ethical opinion}

On behalf of the Committee, I am pleased to confirm a favourable ethical opinion for the above research on the basis described in the application form, protocol and supporting documentation as revised, subject to the conditions specified below.

\section{Conditions of the favourable opinion}

The REC favourable opinion is subject to the following conditions being met prior to the start of the study.

Management permission must be obtained from each host organisation prior to the start of the study at the site concerned.

Management permission should be sought from all NHS organisations involved in the study in accordance with NHS research governance arrangements. Each NHS organisation must confirm through the signing of agreements and/or other documents that it has given permission for the research to proceed (except where explicitly specified otherwise).

Guidance on applying for NHS permission for research is available in the Integrated Research Application System, www.hra.nhs.uk or at http://www.rdforum.nhs.uk. 
Dyddiad/Date: $17^{\text {th }}$ May 2017

Professor Michael J Lewis

ABMU Health Board Research \& Development Swansea University

Swansea University

College of Engineering

Fabian Way

Swansea

Crymlyn Burrows

SA2 8PP

Swansea

01792530888

SA1 8EP

Floor 1, Institute of Life Science 2

Singleton Park

Dear Professor Lewis,

Re: Physiological and physical changes following pulmonary rehabilitation

IRAS Ref: 206031

Sponsor: Swansea University

Thank you for submitting the above named research proposal to ABMU Health Board for NHS R\&D permission. The attached listed documents were reviewed.

Health Board R\&D Governance checks have been completed and passed. Please accept this letter as confirmation of local NHS R\&D Health Board permission.

As part of Research Governance, you are required to:

1. Adhere to the protocol approved and inform the R\&D office and the relevant Research Ethics Committee of any changes to the study, including the end date, for review/approval and record update.

2. For Health Board Sponsored studies, notify the R\&D office of serious adverse events immediately upon knowledge, in accordance with local Standard Operating Procedure on Pharmacovigilance and as outlined in your Study Initiation meeting.

3. For Externally Sponsored studies, the Health Board should only be notified of SAEs or Suspected Unexpected Serious Adverse Reaction (SUSAR) arising in local ABMU Patients.

4. Complete any interim and final reports requested by the R\&D office. If sponsored by $A B M U$ Health Board, you will be asked to complete a 6 monthly progress report for submission to the Joint Scientific Review Committee along with your final report at study completion.

5. Ensure that your research complies with any relevant regulatory requirements and legislation relating to: Clinical Trials, Data Protection Act 1998, Health \& Safety, Caldicott Guidelines, the use of Human Tissue for research purposes, Mental Capacity and ICH Good Clinical Practice (GCP). The R\&D team can advise you on applicable regulatory and statutory requirements relevant to your study.

6. Comply with Data Protection requirements, notably no personal or patient identifiable data should leave the Health Board unless explicit consent from the individual or patient has been taken and documented. Unless consent is present, all study related documents must be either fully or linked anonymised. 'Identifiable patient data includes name, address, full postcode, date of birth, NHS number and local patient identifiable codes as well as photographs, videos, audio tapes or other images of patients. Personal identifiable information includes the member of staff's name, address, full post code, date of birth, NI

Bwrdd lechyd ABM yw enw gweithredu Bwrdd lechyd Lleol Prifysgol Abertawe Bro Morgannwg

ABM University Health Board is the operational name of Abertawe Bro Morgannwg University Local Health

Board Pencadlys ABM / ABM Headquarters, 1 Talbot Gateway, Port Talbot, SA12 7BR. Ffon / Tel: (01639) 683344

www.abm.wales.nhs.uk 


\section{Appendix B: Data accompanying Chapter 4}

\section{PATIENT INTERVIEW TRANSCRIPTION}

(patient 1)

Age: 81

Interview date: 11.07 .2016

Location: residence

Conducted by: Dr. Joan Hudson and Mr. Sameera Senanayake

\section{START OF THE TRANSCRIPT}

\section{Interviewer: What do you think caused your IPF condition?}

Patient: In my younger days there when I started working up the promotion ladder I was a junior charge hand, it was a working position, a working charge hand and one job that I had to do; (it all sound double dutch to you) there was a small ladies section 10, but there was a small course that was made of sand, they are going to overhead they baked to 600 degrees, 34 dive down, if they want to go around twice they twice and they come off. I may have to be sprayed with a mixture of water and coal dust. So, for the time I was being work for the $1^{\text {st }}$ two hours, even though you are given a mask you spit out after two hours it was solid black. But I think I get paid for the job and I did it for the best part of two years, and I think the amount of cold dust I must have swallowed then. That's one thing.

But the last job I had was in BP chemicals. Obviously, we deliver petrochemicals and in one occasion I had a very bad report, where we used to work with Benzene, and at the end of each late shift at the end of the month we would be tested for our urine. I had three tests taken and had results over a hundred and they should be around 100 or less, so they said if that continued, they would have to kick me off that job, but it felt away, and I was alright.

But when I spoke to the doctor down there, I asked what target you really want, was it bellow 100 and he said really, we would like zero he said. So, they really didn't want that in your system at all. So, I think one of those two

Interviewer: So, it sounds like, well I guess you got a history in you know in those kinds of environments that might cause, so were you surprised that you were diagnosed with IPF

Patient: Yes, but they weren't sure at $1^{\text {st }}$, I suppose they suspected lung cancer or either it's a form of lung cancer, they had no idea and I didn't particularly want to know. I've got a friend with lung cancer at the moment. By coincidence, about two minutes before I was diagnosed there was an article on the paper about this young man who was about 45 who said he now can't play with his children and things because he got this, and I read his diagnosis and I thought I felt exactly like that you know and I've never heard about IPF and when they diagnosed me I thought I've got what this chap got and he is only 45 and I was already what? 76. And umm I've had a good run anyway, I mean because my heart, the time I found about my heart was when I was in national service as a young man, because as an apprentice you don't go at 18 and the at the time you finish your apprenticeship you were nearly 21 and I was engaged to my fiancé and she was going to wait for me till I do my two years of national service etc. etc. 
I was fit as a fiddler, I was playing rugby, soccer and I used to do running 400, 800 meter, I was a fit young man, or so I thought. And I went down, and I was grateful. So, they told me that I have a murmur, a leaking valve, and meant nothing to me at the time till I was referred to Morriston hospital, this goes back to 1956. So it was a long time ago and I was diagnosed with a leaking valve of course they didn't do heart surgery in those days, they couldn't stop the heart and um and I had to give a chance to my fiancé to drop me as I was not a very good prospect then, she didn't l'm glad. Anyway, at that time I didn't know I was going to life till 40,50,55, 60 if I was very lucky but at 81 , I am still talking to you.

Interviewer: Ya, really you are still going strong.

Patient: I lost my wife 15 years ago, she had leukaemia. If she would have been there, she would have been a very attractive girl and pretty talented, sang semi-professionally for 35 years. She was a nonsmoker as I. when I say I'm a non-smoker it not that I 100\% smoke, for about perhaps 10 years I know I dint like cigarettes as such but when I have a couple of pints particularly when my wife was singing with her band and I was with other people and ide have couple of pints and my wife had to drive me home, anyways I was enjoying a cigarette or perhaps three but I wasn't an addict, I wasn't addicted I was never ever addicted, I just had a couple of cigarettes. Perhaps I shouldn't have because even that could have caused it.

Interviewer: Well you don't know do you, there are so many factors like you say all the industries that you have worked in

Patient: My wife told me one day when I was smoking, you know you look like a monkey with one of those things, and I stopped, and I smoke never again for 25 years.

Interviewer: if you were addicted you could have never stopped like that.

Patient: I'm talking too much

Interviewer: No that's ok, that's what we are here for. That's lovely, we are really interested to hear. So, when you were diagnosed it was still a surprise.

Patient: Very much a surprise. Particularly by confusedness in to what it was exactly. Of course, I soon learned and told them don't hold back, I don't want to hold back, I want to know what it is as it is? And they said they don't know. And I said what about this article, I read that article and later on I saw another article found on internet and I bothered my children to do it and I've read enough to realize that on average there is a three year life span and some are lucky and get a bit further and others have 8 months. So, I knew it was a wide scope that I've got. Anyway, I'm glad to say I've got lot of friends and when I was diagnosed, I was struggling as I have never been struggling with walking and it coincides with onset of this one. And I'm thinking it may be this which is making my walking difficult. And I told I've been given a bad diagnosis and I have a limited time, and some say you are bloody around $\mathrm{u}$ said you are going, I'm glad that I'm not but sometimes days I don't mind going.

Interviewer: So, u said a few things which were quite interesting there, so when you $1^{\text {st }}$ got your diagnosis and you found that it was a varied life span, how did you feel when you heard the news about your condition? 
Patient: I was determined to live my life for the fullest, for as long as possible. By this stage, I said I've lost my wife 15 years ago, after a couple of years I was terrible and I lost my way, I had to be treated for depression and I started out I was missing the ladies company, I'm not about the physical side, I had a couple of relationships one was almost five and a half years. I was quite surprised by the diagnosis and that there was no treatment at all. I thought surely there is something. I'm on painkillers now but those are for my back not my lungs, they may be allied, I have no idea. It is surprising that there is no treatment as such and now I am more aware about the situation particularly after joining this group (support group). It is nice actually to meet some people with the same disease, I thought at least I was not alone. But when I was up there last Monday, I couldn't help thinking that this is like shipman's waiting room, waiting for each one to pop off you know. Because there is always news that somebody is gone and that's a person u know, and I think I my name would be there one day wouldn't it.

Interviewer: Ya I mean the fact that there is no treatment for it, did you kind of deal with that in a certain way? Have you tried to approve things?

Patient: I understood the few treatments that they did have in mind like lung transplant and etc. it was completely out for me at my age anyway. And with the new laws to transplants from people, I'm for it personally, because if you can save somebody else's life you should.

Interviewer: So, I mean u mentioned previously you are a sportsman and you are very active and so on. And you mentioned you use your walker now, how does the condition has affected your daily activities?

Patient: Oh, greatly in fact. My dog died two and a half years ago but one of the delights of life was walking my dog. I think he went at the right time because I'm not able to now. I couldn't look after him, I couldn't brush him so in a way I'm glad that he went. But that aspect of my life, just going out for a walk no problem up until six months ago. I still drive and once I'm settle down, I'm ok and once I get to somewhere, I have to plan everything. Even looking after myself here. I don't eat a lot at home because I eat with these ladies, but I would be doing lunch my self today. And I my dishes and I try to avoid walking as much as possible. You got to be thinking all the time you know and that thus affect me. And another thing, because of my interest in sport my wife took me bowling and I was bowling up till two years ago three years ago and I joined the club eventually for about five years and I miss that the companionship. My son-in-law is the captain in the team, and I would have loved to play under him. But I'm still, I've got a season ticket to the swans and I'm going there by my wheel chair because l've graduated from an excellent seat up in the stand to lower regions until they found me a spot on the side, and I've been lucky, I've got a good spot there and I've been watching them for the last two seasons. I've got a ticket for the next season but weather I'm there to see it or not, I don't know. One of the things I've found particularly after losing my wife l've never got board because l've always got things on whether its singeing, sports or general life I've never board been, I think the boredom will kill you. That's why I don't like staying in too much. I honestly feel that it has helped me because I would rather go with a band than sitting here waiting for it.

Interviewer: you've been talking about staying active and looking to the future and planning things for the future. I guess it digs in with couple of these questions, on a day to day basis how does having IPF make you feel.

Patient: I attend chapel every Sunday morning, I'm not the best Christian. I've had heart issues since I was 30 and even for this, I don't feel paranoid, because I keep thinking about other things. I've started 
staying in a bit more. But I get up night to go to the toilet and I get pain I don't think it's IPF, but I might be wrong. I went to a course of physiotherapy and honestly, I think it's too much for me, when I came home, I was knocked out I wasn't even able to do simplest things. Now my strength is gone and sometimes I feel ashamed about the things I can't do, perhaps it could be because of getting old.

Interviewer: I want to come back to the support group. But you've mentioned the rehabilitation programme, so when that was proposed to you, your $1^{\text {st }}$ thoughts were that's too much I can't do that?

Patient: No actually, I thought my input will be valuable because the people there are surprised that I've lasted five years. And I thought I can't actually take part in physiotherapy, I'm pretty sure I can't also because of my heart problem.

Interviewer: you mentioned the support group and you mentioned some positives and negatives being involved in that

Patient: The positive is that I've met people slimier, some of them are very nice. There was this one lady who I felt I really cheered her up. I would imagine she is approximately my age and I said to her I've been diagnosed for five years. And she said $h$ Fred had it for three years (her husband) and I felt that she's thinking that there is hope for Fred for another couple of years. So that's the upturn. Downturn is every week they announce the deaths to me. And that is a bit of a down turn because $u$ all know that you are going sooner than you like to be. Another down side I felt last time I was there. I went there fairly early and as people were coming in a chap came and sat with his wife and it was his first time and he wasn't getting involved and I thought I felt like that in my $1^{\text {st }}$ time and I didn't feel that good coming out of that one because I thought do I really have to come here and perhaps get depressed. But I kept on going because if we could do anything for 20-30 years down the line...who knows.

Interviewer: you said a couple of things that I just wanted to follow upon, you said about you don't like to live with it, but you have to live with it, I'm just interested to know how do you live with it? Is that a question you could answer?

Patient: Putting it out I don't mind, oh no that's wrong, I do mind it every day. Challenge is living with it. As I described earlier, finding the simplest way to do things and I try to keep everything in precise order. So, things at the same place all the time. Matter infect the lady who come on Friday said you are so obsessed with everything, but I find life is easier that way. That is also because of my mind and I'm a great believer in keeping the mind occupied, I mean I'm not a couch potato when it comes to telly, but I love watching interesting programmes.

Interviewer: going back to the support group, the thoughts that time when you went and thought you didn't want to come again. Was that because it reminded you how you feel, you know about your condition?

Patient: May be, it's just the days because the feelings were too varied. I got days where I'm feeling reasonable and life is full of hope and there have been days where I thought I won't see tomorrow when there is no company around me, those were the down days. I didn't have lot of those, but I don't get deeply depressed though, it's just that accept the fact that my time is limited but sometimes going to support groups brings some hope to you, you know because everyone else's is time is limited. So yes, I'm glad they got it and I certainly will support it, I might miss out occasionally, but I will go as long 
as I'm able to and it surprising that new members are showing up all the time. So, it's a good set up and yes it been ignored probably because many medical professions don't understand it. They don't know what is causing IPF, they are guessing its work, smoking, genes, life style, I've always enjoyed a pint but I'm not a boozer, I've never been a smoker as such, and I've always been reasonably fit.

Interviewer: You talked a little bit about lack of awareness in medical profession about IPF, what causes it you know and so on. How does that make you feel, the fact that there is so little understanding and knowledge of your condition?

Patient: The patient did not give an answer relevant to the question

Interviewer: You know, you sorted mentioned that it's an untreatable condition has anybody suggested any ways to help you manage it to make life more comfortable and prevent the decline?

Patient: In a way yes. Because I've learnt in two occasions in the last five years, I've had people come and install things like grab handles in my shower and alternate steps from a larger step to a smaller step, those type of things. Things like that help you with life. This thing was given to me (walker). But do I like too much of items, No, but I'm old now and I can't get around here without any support. And I've got two alternate sticks if it is in and out of shops or the club. I got a walker and a wheelie, and I've got an electric one out there, second hand I've bought it and I would love to go around doing walks with my dog,

\section{Interviewer: do you rely on other people?}

Patient: I've got a gardener, he was meant to come last Thursday but he hasn't. I keep the back gate open, but I don't like doing, in case he turns up. And then I've got two daughters and a son, they are always there if I call them but one of my daughters is in Scotland, but she would be down in the next plane if necessary. So, I'm not short of support. I'm very sorry for people who haven't got back up and I've got a couple of those.

Interviewer: you mentioned your family; how did they support in your condition. Did they encourage you to do anything to look after yourself?

Patient: They've always said to me, I would never have to worry about being on my own. But I want to be on my own as long as possible. Even as you get older you like to have your independence. I like to feel the king of my kingdom. They would do anything if I ask my family, but they prefer me to ask them because they know what I'm like, I'm stubborn. I couldn't have asked for a better daughter in law for my son, unfortunately she can't have children, there in their 40s now and she is excellent. She always cared about me. And l've got these systems, my daughter who is a teacher sends me a text "OK Dad", and she knows that I'm OK. So there caring and we got these system because there is nothing worse if you collapse and you are on your own. So, the family is so good being not pushy.

Interviewer: I just want to ask you a few questions about pulmonary rehabilitation, so that was something that you felt, you don't have the strength to do?

Patient: I don't think so 
Interviewer: what's your view on what it includes?

Patient: Going by what I did with the heart, I guess they are using some weights, running or semi running and the rowing machine, bicycle and all, I enjoyed doing all that, and it did me good and I know that it will probably do good to me now.

Interviewer: I think, the rehab programme, if you were offered that while ago, right at the start of your condition, do you think you would have felt differently?

Patient: Having a look back most patients haven't got my back problem.ve got these elastic bands at the back for exercise, I don't do it to be honest because I'm already overtired to what I am. So, I think it's a case of yes it would do me good or it would, or it will finish me off. But if it was for other physical things, I would be the $1^{\text {st }}$ to say yes

Interviewer: when you were talking about different types of exercise that you did in your cardiac rehab programme and then you talked about doing seated exercise with your therapy band, if you were to design a rehab for programme for people with your condition, what would you recommend that it would include?

Patient: Some patients are 40-50 and most of them are fitter than me. I think it's a good thing and I think people should be encouraged to do it. And if it was 10-15 year ago, I would have given it a go.

Interviewer: are there any other thing that you think will be useful to include? I mean you mentioned seated exercise, is that something that would be helpful?

Patient: There are quite a lot of exercises that I know that you can do sitting down. People might be put off coming or might be encouraged by sit down for these things, there are plenty of exercises that could be done sitting down.

Interviewer: I suppose this is a bazar question for me to ask, but obviously it's an untreatable condition and the exercise won't cure it so from your point of view, what would be the benefit of exercise with somebody with IPF?

Patient: I think it will prolong your life, because the fitter you are the more it's going to help your lungs anyway. There are lot of people who don't realize that. The other side of the coin I've seen people dropping dead during their walking, and they are quite fit people. So, it doesn't automatically mean if your body is fit you are not going to pop something else. But basically, I do subscribe to keep as fit as possible. I've done it by not necessarily doing exercise but being active (doing daily activities like cutting grass). For those who would say I want to sit down, certainly there is enough can be done.

Interviewer: and part of the rehab programme is kind of education about IPF, you've had IPF for 5 years, which is a long time, what kind of things do you think will be useful to educate people?

Patient: I'll show you, because I write to different charities (shows an article on IPF by BTS). You know that was sent to me, it explains what it is and implications of it. I think something like that could be handed to people so they would understand it, particularly in the early stages. 
Interviewer: so, do you think information is useful, and you've got a case study here on somebody called John, he's got IPF. Was that helpful for you to have read?

Patient: In the early days when I knew nothing about it, I thought it was only matter of years with it that would have been very helpful, as some people are. Lot of people need to know what the disease is and understand it and they can accept it then. You understand the news and you know you won't be around in 7-8 years' time. Unless it's somebody who disintegrate with the news, as long as people know the full facts, you could expect it, life with it and live accordingly. I lived normal as I could.

\section{Interviewer: what would be your advice to somebody if they were diagnosed today with IPF?}

Patient: You should find out more facts, face to the facts, accept what is inevitable and live accordingly. Whatever you want to be, if you want to be cocooned in the house watching telly so be it. I don't subscribe to that but try to keep your mind active as well, as I always believe an active mind helps the body.

\section{Interviewer: Do you think having IPF have changed you as a person?}

Patient: It's a damn darn question. I like to think, I've got lot of friends who show lot of affection towards me, and certainly I'm bit shorter tempered now, partly because I feel bit of a grievance that why this after all the years of struggling with my heart and everything. I think I'm the same person I used to be. Lot of people like to see me especially the ladies.

Interviewer: as an IPF patient was it easy for you to get hold of all the information? How did you find that process?

Patient: $1^{\text {st }}$ of all there was that paper which gave me insight before I was diagnosed, I've never actually been given written information other that what I have picked up myself. Information is good and eventually more people would understand what this is. Lack of information always causes more worries thank being told the hard facts. No one has given me enough physical advice on it. Not everybody wants to be known but I do.

Interviewer: Thank you for taking part in the study

END OF TRANSCRIPT 


\section{PATIENT INTERVIEW TRANSCRIPTION}

(patient 2)

Age: 80

Interview date: 14.07 .2016

Location: Swansea University Bay Campus

Conducted by: Dr. Joan Hudson and Mr. Sameera Senanayake

\section{START OF THE TRANSCRIPT}

Interviewer: can you tell me a little bit about the onset of your condition and a little bit about it?

Patient: looking back I think probably about three years ago, we were on holiday and then um, and I happen got a chest, never thought more of it. And then we went on a cruise, 2014 February/march and again I had a chest problem. And we went to Spain, I had a really bad chest there and I was putting it down to picking something up from travelling on the plane. I went to see a chemist and she prescribed antibiotics, which took and when I got home, I went to see the doctor and he was a bit off and asked why I hadn't gone to see a doctor in Spain. And I said well I've seen the chemist which was free of charge and she did the job well, she gave me the anti-biotics which worked but I came to see you as my own doctor. So anyway, he checked me over and he wasn't sort of very keen on going any further. My wife was with me at the time and she said this has happened two or three times, so in the end he decided that I should go and have a chest x-ray. Following the chest $\mathrm{x}$-ray at the singleton hospital and the consultant referred me to Dr. Harrison in Morriston Hospital. When I saw he came out and said I have pulmonary fibrosis. And since then I've been seeing him fairly regularly.

\section{Interviewer: and then did you get much information about pulmonary fibrosis?}

Patient: I firstly understand that there is no cure, it's a disease which lot of people don't recognise and my doctor was a little short at the time but after he found out that I was diagnosed with this he was exceptional after then, especially at the time my wife had a brain tumour and his attitude from then on was absolutely fantastic.

Interviewer: but initially is was like a mystery it sounds like?

Patient: It was a mystery, he didn't think somehow, though I was complaining about chest, I know one thing I've missed out a lot now just thinking back, a year last September (2014 September) we've gone to Madeira and we were swimming normally, and we are both good swimmers and I would swim behind my wife and being a man I would always have to accelerate and pass her, but I found out that I was struggling to do a length. That's I've supposed why. I went to see the doctor and said there is something wrong because I've never had any trouble swimming at all or distances, that was the main thing you know

Interviewer: so, you were quite active before? 
Patient: Oh yeah, I used to until this, I'm on crutches now for the last three years and I've had balance problems before and I used to play a lot of golf, swimming, cricket you name it. I've had no problem, but now I'm a television viewer.

\section{Interviewer: so there has been a change in your activity's levels}

Patient: I find I'm tired if I got down to put my shoes on, I become breathless. The other problem is my balance and walking and I find it a great struggle in the last two years I've gone from a wheel chair which I use, because when we are away with my friends I was slowing them down, so I got a wheel chair and my wife had a problem pushing it because she had arthritis in her hand, so I got a scooter.

Interviewer: other than the way you get around in places and getting breathless when you bend down to do your shoes, how else has it affected your daily activities?

Patient: I would say, people would say I am lazy but when I walk to the kitchen now I leave plates, knives and everything, where I'm preparing the meal, I'll leave them and wash them all in one go and put them back, rather than put them away because I'm not going to get up and walk 5 yards to get the plates and come back. I've sort of try and do everything in one place so I don't go somewhere and I would have to back for something, can't be helped but sometimes you forget, I sort of, it's like I go in to the lounge and the kitchen, I always go in the bathroom because I think of getting to the lounge and I don't wana go back because it not on the way, it's sort of reorganize your life, try and do everything in one place before you move to another.

\section{Interviewer: is there any other way how it affects what you do and where you go?}

Patient: The next doors neighbours invited me few weeks ago on a Saturday night, because I was on my own at the time and I was coughing quite a bit which was a bit embarrassing and it seemed to persist, and I thought this is no good but um I couldn't do anything about it. That's the problem, I find quite often at night I start coughing and sort of like there is medicine. The only thing that I found helpful is menthol sweets which seemed to help a little.

\section{Interviewer: Is it only you in Swansea or do you have your family here}

Patient: no, I'm on my own here. (Rest of the answer is deleted from the transcript because it was off topic and irrelevant)

\section{Interviewer: What does have IPF mean to you? As an individual.}

Patient: um, I suppose really, it's just something carped on over the years. I've always like doing everything by myself. I've always been very sporty, now I don't go golfing, I don't play tennis, I don't go swimming, it's limited that side, the only thing is I can walk in water because l've got crutches so I'm OK in the swimming pool, but I suppose now I need somebody with me and I'm general it's sort of, wouldn't say its lazy but I tend to leave things for a bit till they all get together and as I'm on my own now I just please myself. It's like I don't make the bed every day because I can't stand so I leave it and I would leave my clothes on the side of the bed at one end so they are ready for me to put on in the morning (Rest of the answer is deleted from the transcript because it was off topic and 
irrelevant). I suppose everything is very slow, but one has to accept that's the problem and make the most of it.

\section{Interviewer: How do you make the most of it?}

Patient: I suppose, since I came back from Canada, there has been a lot of sports on, so I've tended to watch that and left things which is needed to be doing, these days there is not much in the television now so I'm getting on with my paper work. Last few days I feel a lot better about myself. I've seen Dr. Harrison three months ago the day my wife passed away. (Rest of the answer is deleted from the transcript because it was off topic and irrelevant).

Interviewer: Is there anything positive come out from the diagnosis of IPF?

Patient: Difficult to say isn't it. I've lost my wife and I'm still alive. So that's positive. (Rest of the answer is deleted from the transcript because it was off topic and irrelevant). When people see you coughing, they say take cough medicine, take this take that, they don't understand it.

Interviewer: Does it make is sometimes difficult then, because people doesn't understand it?

Patient: Because it's invisible, they think, or get some cough medicine or do this and that, when I called Dr. Harrison it was completely different. I don't think they believe there is anything wrong with you in a way but quite often they would say why you are panting when you are doing nothing literally, or you don't realize you are.

\section{Interviewer: How does that make you feel?}

Patient: When my wife was in the care home and I used to sleep and I used to have a phase about two weeks when I felt out at night, as soon as laid down to go to sleep, I was getting sort of, I couldn't breathe properly or had that feeling that I wasn't able to get my breath which I found difficult to handle for a bit. That's disappeared now. I didn't know if oxygen was working at the time, but it is worrying when you are on your own. Every time you lie your head down and in 10 minutes you can't breathe properly and it's a bit worrying, and you wonder if it will get worse.

\section{Interviewer: What is your understanding in pulmonary rehabilitation is?}

Patient: I've had a leaflet from lung association and about exercise and I have not done anything about it now. There is one in Swansea. I do go to a physio class, I go in twice a week now doing regular exercises and I can agree with that. I can see doing all the exercises without being a couch potato and it's good.

\section{Interviewer: so, what kind of exercises can you do, could you give me couple of examples?}

Patient: yes, you do march on the spot moving your legs to each side forwards backwards, starches and walk one foot in front of the other which I can do with support. Some of them I can't do like I can't get on my toes or heals but that due to my balance problem. They work through your whole body like exercising your arms use the tuber to put pressure to your joints, especially to your arms and wrists. 
You do exercises where you do stretch out the tension and hold for five seconds and repeat it for 5 times. I can understand it and what it is doing.

Interviewer: When you said it was doing you good, what type of benefits do you think you got?

Patient: I can't walk very much, everybody says you should walk here walk there so it has given me exercises to do with 10 or 12 people and you tend to say right, l'll do it all I'm not going to sit down you know not give in, otherwise in sitting in all the time. I can't go out for a walk, I'm struggling with my crutches and the idea is that it will be tired you for a bit and get the heart rate going and get your lungs working and everything.

\section{Interviewer: Do you think it's kind of helping with your condition?}

Patient: It could be because, I don't know how the condition goes and the leaflet you have given me is very nice, it says $50 \%$ only make it for three years, mine is staying static at the moment. It's nice to get a reading which confirms you are not deteriorating. And looking back my wife and I took a full MOT few years ago and I didn't take any notice of it but they said there is spot on your lung which could have been the beginning of it, as I thought I don't smoke and I was fine for years afterwards but it come on in the last 18 months, it must have been there and the last few months I have been at a point where nothing is happening. But I'm reasonably happy ATM.

Interviewer: do you actually enjoy the exercise sessions that you do?

Patient: yes, the only thing I find, the original one I went to was run by NHS for 8 weeks and at the end of that you pay as you go, I think a couple of pounds. The $1^{\text {st }}$ one had tea and biscuits and at the end of it you get talking with other people, especially if you are sort of a single person in your own it's nice to have some company. But the second one there are no company you are on your own. It gives you something to look otherwise you will be sitting in home all day.

Interviewer: If there was a Morriston would you be interested to attend?

Patient: yes

Interviewer: what kind of think would you like in it in terms of exercise?

Patient: well, I' $m$ in the dark about what you can do to help the condition and I'm open to suggestions you know, anything that might help is going be good.

Interviewer: What would be the things that would put you off going like exercises as you said?

Patient: Nope 
Interviewer: are there any kind of other exercises that you like to do, or environments may be like mixed groups?

Patient: yes, it doesn't worry me I mean in the past I've done exercises and I've been the only man around 10 women.

Interviewer: You are open to exercise so how often do you think you can manage doing exercise on a weekly basis?

Patient: I've only started to go for the physio twice a week instead of once. But to me pulmonary one would be more important compared to the physio one so I would choose pulmonary over physio. Rest of the answer is deleted from the transcript because it was off topic and irrelevant)

Interviewer: Do you think there would be wider benefits doing pulmonary rehabilitation?

Patient: It's meant to make your breathing easier whereas the other one is a more arthritic one on joins and muscle.

Interviewer: You mentioned your family in Canada, do they have any view on about your activity level?

Patient: no, they have never said anything at all. You can't expect me to do a lot, if I need them, they are at the end of the phone. But they have their own life.

Interviewer: If you can see yourself going on the pulmonary rehab programme, are there certain things you look forward to or something might worry you?

Patient: I don't think that anything will worry me. I've always thought myself as somebody who doesn't worry, and things happen in life and you get on with it.

Interviewer: because you weren't aware of the pulmonary rehab programme, you haven't talked about it with anyone?

Patient: No. the only thing I got was a leaflet from British lung foundation saying about classes but that's it

Interviewer: when we talk about pulmonary rehabilitation there are different components like exercise and education. What kind of things would you like to see in it or is exercise is just enough to you?

Patient: to me exercise seems to be the only thing. Unless it is explaining the problem, what's causing IPF there is no point. l'd rather be told things you know. 
Interviewer: If you were given a choice of doing an exercise sitting down, would you...?

Patient: I find it easier doing exercises sitting down.

Interviewer: so, sitting down exercises would encourage you to attend?

Patient: yes probably.

Interviewer: looking back in life do you think may be something that you did caused your condition?

Patient: Dr. Harrison sort of dug in to this. I had a family motor business and I thought looking back in the sixties when you look at the breaks, when you take the brake drums off and you have asbestos and you blow it off and may be when u paint cars u cam puffs it. He said it doesn't cause IPF and this came 40 years after I breathe asbestos (the spot on the lung).

END OF THE TRANSCRIPT 


\section{PATIENT INTERVIEW TRANSCRIPTION}

(patient 3)

Age: 81

Interview date: 14.07 .2016

Location: Swansea University Bay Campus

Conducted by: Dr. Joan Hudson and Mr. Sameera Senanayake

START OF THE TRANSCRIPT

\section{Interviewer: You're IPF, when did it $\mathbf{1}^{\text {st }}$ got diagnosed?}

Patient: well, I was diagnosed year last May. Year last January / February, I was with my doctor. Think all this after losing my wife in July. I didn't let the grass grow on my feet because I've never been too morbid. In October I went to north of Organya but this year it's so bad I can't go anywhere. But as I said I was going to the doctor in February last year and I said I want to see a specialist and they refused so I booked to see a consultant and on one morning I got a call saying we had a cancellation and a spot opened up so I went to see this doctor in Morriston. He checked me up and said I have clamping fingers, got x-rays and said I think you have IPF. I have never heard it before, so he arranged a CT scan and then saw Dr. Harrison about a year ago.

Interviewer: it has affected how mobile you are, has it affected your other daily activities, way you do things around the house?

Patient: Oh, I can't do anything. (The rest of the conversation was deleted from the transcript because it was irrelevant to the question).

Interviewer: driving long distances with your oxygen on is quite difficult for you?

Patient: never tried it. But if I was driving on a cushiony seat and I'm only driving and not talking. This is what rehabilitation is going. If your muscles are fitter you need less oxygen, this is what they do there strengthening muscles and it marvellous.

Interviewer: so, you have been doing the rehab exercises have you and for how long?

Patient: I've done that. About six weeks of it but I lost 4 days, 2 weeks.

\section{Interviewer: how did you find it?}

Patient: well, as I said to you earlier what they teach you are the basic exercises to keep your muscles toned up. By toning your muscles up, you get stronger muscles and use less oxygen up. It's easier said than done sometimes, if you are struggling for it. I mean a year ago I was doing all the grounds on my tractor cutting grass but this year I can't do anything. 
Interviewer: what kind of exercises did you do in the class, what could you manage?

Patient: we would sit in a chair, they put weights around your legs and lift it up like that and you stand up and sit down and you step up and stretching and bicycling. I did what I could.

Interviewer: did you enjoy it?

Patient: yes because, I found it struggling but as they said to you do it to your own phase. They never pushed you. Yes, and it was about meeting other too.

Interviewer: some people actually do not go to rehab programmes, what encouraged you to go?

Patient: I told myself you have to try anything and everything. I have an oxy meter and I check it during my activities.

Interviewer: when you were doing the exercise session, do you ever worry that your oxygen levels would get too low.

Patient: no because I'm on this and if I feel like it, I turn it up a bit and I take a full cylinder when I go.

Interviewer: you said about doing exercises, you know doing what you can. Do you think it helping your condition other than the extra oxygen going in to your muscle?

Patient: I don't think so. I just don't know. I know the difference, from last year it it's a huge difference and I've gone down.

Interviewer: How do you fell having to use oxygen all the time?

Patient: I've got no choice you know. It's like a tie. You are stuck at an end of a tube really. I try and manage without it. I don't want it any more than its necessary you know unfortunately. I don't sleep with it, so I go down quietly.

Interviewer: how does your family help you? Do they help you to manage your IPF?

Patient: My daughter came in some time ago and helped me to slip in to bed. (The rest of the conversation was deleted on patient's request).

Interviewer: going back to your family, do they encourage you to be active or do they say dad sit down, take a rest?

Patient: well, it's not a matter of encouraging me because they know what sort of a person I am. If I can do it, l'll do it. I'm a very independent person. 
Interviewer: what do they think about pulmonary rehabilitation, do they think it's a good thing?

Patient: I don't know, I haven't talked to them about it. They know I'm going, and I have finished it now.

Interviewer: having gone through that programme. Do you think its missing anything? Doesn't necessarily have to be exercise, may be lifestyle or diet?

Patient: No, because you see, you did an hour of excising and then you are sitting down having a cup of tea and biscuit and chatting with others. There was a dietitian there and I saw her because I got a stomach problem as well. I don't know if it is affecting. I had that for a long time (rest of the conversation was deleted because it was irrelevant).

Interviewer: do you believe that something u did caused you IPF, like smoking?

Patient: never smoked other than a few cigarettes as a young boy. Never was a drinker, drank very little. If you google IPF you would find acid reflexes could cause it and it makes sense to me because I've suffered from it in the 90s. So maybe it's a contributing factor. I don't know

\section{END OF TRANSCRIPT}




\section{PATIENT INTERVIEW TRANSCRIPTION}

(patient 4)

Age: 71

Interview date: 14.07 .2016

Location: Swansea University Bay Campus

Conducted by: Dr. Joan Hudson and Mr. Sameera Senanayake

\section{START OF THE TRANSCRIPT}

\section{Interviewer: How were you diagnosed with IPF?}

Patient: Here comes the start of the problem, In February $6^{\text {th }}$ of February I fell down the stairs from top to bottom and went to Morriston hospital. They were going to operate, and they found me not fit enough to undergo anaesthetic and I was in there for a month. Mr Harrison did a multitude of tests and eventually I was diagnosed with IPF. Gave me something like a short period. It was like months or three years to live. Because it came so quickly, and I had no indication that id suffered from this condition. My background is working in an office environment not the same office, but different places for 26 years. I was in for a month, I have oxygen in my house and in my car and after a month I came out, knowing I have possibly a short period to live, I started to arrange the inevitable, but I also took to drinking too heavily and end of June I got jaundice. By September I finished taking oxygen and then I started the rehab in September same year, three years ago. It was 7 weeks twice a week (14 sessions). I found it extremely helpful. And that's the introduction.

Interviewer: so, you've been through the programme, what kind of experience to you have, the positives, and the negatives?

Patient: I didn't have any preconceived ideas. I had no idea what's it going to be like. They said it going to be a light weight circuit training. It wasn't the important thing, I think. It was mentioned that it's not a competition although there were around 10-15 people, you would do exercises to your phase. Yes, you were trying to do routines of 10 pullups but if you couldn't do it you do as much as you could. It wasn't to gain strength, but it was to exercise your lung and mussels to help your blood and air to go to the lung. There were people who supported it. it must have been very good, well I know it was good. Because the dropout rate was minimal. If you miss a day people were coming with doctor's letters the next day. All thought they should participate. It did get a group of people working to gather with similar conditions. I prefer to say a condition rather than a disease because it sounds less when you say condition.

So, no preconceived fears and the results were good. Initially your heart rate and oxygen recovery time were taken, and they were periodically recorded just to see if you have improved and most people did, so I was more than happy to participate. But after I completed you couldn't do it again and I guess that's a draw back. But I was referred by my GP to group down in Port Talbot. There were all of mature age and was having COPD. It wasn't a gym, but they had dumbbells and weights and it was professionally run, so we did that twice a week and I've been doing that ever since. I find lifting weights easy but anything to do with breathing like waling, I find it difficult. 


\section{Interviewer: you don't exercise regularly now}

Patient: yes, because I'm out of breath when I do. (Rest of the conservation was deleted from the transcript because it was irrelevant). The exercise programme had 14 work stations, 2 minutes at each station which alternate from chest, breathing, legs or arms and it works. All the participants were elderly, and it was great listening to them. It's important as well as the exercise because we were all suffering from the condition COPD or IPF and again people like to come talk and socialize and mentally it helps as physically. You don't feel like you are doing for the exercise but to meet other people. I find that rewarding and everybody in the group find it satisfying as well

Interview: So, do you find other mental benefits, so is that kind of social connection element that you were talking about?

Patient: I'm not a words smith, I'm more figures and maths. I love the gardens, but I can't go much gardening any more, but mentally I feel good. I do loose concentration when people are talking especially when they are talking about death.

Interviewer: can I ask how old you are? Sorry to be rude.

Patient: I'm 71 . We had our $1^{\text {st }}$ granddaughter last October. She has a quite a character now. So, exercise from rehab I would highly recommend.

Interviewer: would you go for rehab if different types of exercises were offered? You know you talked about warming up circuit training and cool down. Is there kind of other exercises that you would feel you could manage better?

Patient: I don't know what is available. Seated exercise may be beneficial. I don't want it to be one to one because you take away the group feeling. But I will be going back on Monday with my oxygen and I find it easier to do exercises with my oxygen on.

Interviewer: How would you encourage the other people to take part in pulmonary rehabilitation?

Patient: initially I will direct them to our support group. Then I would ask someone who had experience in the condition and rehabilitation programme to talk about rehab, who can pass on their true feelings not a clinical thing, somebody talking from the heart who had done it.

Advertising- there are lots of leaflets with the doctors regarding lung cancer and diabetes. But there seems to be little knowledge in advertising IPF. Maybe it because it only effects a small group of people. Notices in hospitals would be good. Not making feel frightened from it, it's not a competition, u r not to achieve a certain level but any exercise is good for you. You should be encouraged not forced by the trainers. You shouldn't be the bust, but you should be doing your best

Interviewer: it sounds like you have started the rehab very early on when you were diagnosed, you think being active all that time had helped keep you avail?

Patient: that is true. I don't know. 
Interviewer: How does it make you feel to have a condition that there is very little information about?

Patient: privileged! I've got a rare blood good. Feel special. I don't think why me. There are positive things a million things that are good. And this $s$ going to be resolved in years in the future although it's now untreatable. I don't have bad days. I have good days and not so good days.

Interviewer: in terms your family, what's their view on you exercising, are they supportive?

Patient: Oh, very much so. Extremely supportive. My wife says there is nothing wrong with me, but she supports me. I love cooking, doing the prep work but I get tired, but my wife supports me because she knows I love cooking. My grand child can sit on me, but I can't lift her up, is surprising because I can lift dumbbells.

Interviewer: do you think that you did something that provoked this condition?

Patient: I smoked. One a day and was up to 15 a day and 20 a day in the weekends. Then I gave up. My office environment was a non-smoking place.

Interviewer: Thank you for coming today

Patient: we all know we are going to pop out one day, so be it but life is good as we are living it.

\section{END OF TRANSCRIPT}




\section{PATIENT INTERVIEW TRANSCRIPTION}

(patient 5)

Age: 65

Interview date: 19.07.2016

Location: Swansea University Bay Campus

Conducted by: Dr. Joan Hudson and Mr. Sameera Senanayake

\section{START OF THE TRANSCRIPT}

\section{Interviewer: could you tell me a little bit about how you were diagnosed with IPF?}

Patient: it was in 2008, didn't realize I had a problem it was found by chance because I had an operation in my eye. Before the operation the anaesthetist checked me and said you have a rattle in your lungs and you really need to get it seen to. So, it was diagnosed then. And I've been attending the clinic ever since every six months. It was an oddball in some ways because the results of the lung function tests hardly changed. The Dr. was expecting to see deterioration and it doesn't appear to have happened, thankfully. When he sees me, he says I would expect to see deteriorate with your age. I'm 74 you see. And he said I'm not seeing that. From the beginning its being don't change anything. But I put stability down because of the exercises regime that I'm doing, which I have always done. I don't go to the gym, I don't pump ion but I'm active in other ways. I go for a walk for two hours with the dog and its quite tuff walking, you couldn't walk in shoes, you need boots, and it's that sort of walking. And when I do walk with people who haven't got the condition, they get you know. So, it's kind of vigorous work.

\section{Interviewer: so, you didn't have any symptoms beforehand?}

Patient: I was having some Flem. But not severely though. And there was a slight cough. Other than that, I didn't know really. I was never really out of breath. Also, with the lifestyle I mean, now widowed and just having finished work you can imagine how busy I am from Monday and Friday, with the grandchildren, those two days are blown away. I've been working Tuesday, Wednesday and Thursday and that left me Saturday and Sunday to do pink jobs and blue jobs you know. Where previously I was doing blue jobs. There are also lot of house work and stuff like that. So, I was busy. I've got three children and they are all in the 40's and I have five grandchildren. And I'm seeing them quite regularly.

\section{Interviewer: what do you do for your occupation?}

Patient: I was working as an engineer (a sales consultant) and I was only working for this company for eight years and they offered me a job when I was 65 and I retired.

\section{Interviewer: before that what did you do?}

Patient: for lot of years I was hands on machining and for a number of years I worked with graphite. If I was not near a machine, I was near a machining facility and there is a lot of dust there. This is manmade graphite with lot of resins and oil. 


\section{Interviewer: did you wear a mask?}

Patient: Not really, I mean we did but no one did it really. Because it was carbon at it was meant to pass through your system. Whether that was the cause of the damage, I don't know, maybe not. It would be interesting to know about other employees in the industry. (The rest of the conversation was not included because the patient was talking about graphite).

\section{Interviewer: when you were diagnosed were you given any information about IPF?}

Patient: only that the disease won't go away. Its life threating. I've seen life expectancy is between three to five years. But it's not my case thankfully. I remember asking Dr. Harrison if the results of my lung function test are stable, why would you want to see me every six months? And his words were it's a disease I won't trust. I also understood it could be caused by really any foreign body. (The rest of the conversation was removed for the transcript because it was irrelevant). I smoked up until 1969 and in the late 90 's early 2000 both my sons were smoking cigars and I would have an occasional cigar, but other than that I never smoked. I've never had a lung injury but had asthma as a child, but it went away. I don't think there is enough information out there to make people sit up and think about it and prevent this.

\section{Interviewer: when you were diagnosed, how did you feel about that?}

Patient: I didn't get particularly bothered you know. I thought if that is the way it is, I should give myself the opportunity I can, stay fit which I have done. And since my wife died, I have lost 10 pounds. I thought it was grief, but it never came back. It doesn't bother me, I think one of my fails is I have a flat scotch and a glass of wine, I guess that's my only health fault. I don't take any medication, I've got small aches and pains. Last 18 months I have had 5 operations and that itself could be a trauma. But other than I'm It, tip top.

\section{Interviewer: so, your life style hasn't changed much?}

Patient: no

\section{Interviewer: has it affected what you are able to do?}

Patient: I haven't tried running so far. Every now and then I would go up the stairs and I would think woof, I would feel that. Back in February was one of the worst times with regard to this. I felt really sorry for myself. I think I had a chest infection without any other symptoms. I was walking the dog and I wanted to go home. It was never like that, I could see myself going down a spiral and it lasted about a week. So, I was feeling sorry for myself but other than that it never bothered me.

\section{Interviewer: what does it mean to you to be diagnosed with IPF?}

Patient: as long as my results are normal, I feel that I'm on top. Sometimes it could be a little depressing like during February thinking is it gona incapacitate me you know, when life ends are this what I'm gona die from, but overall not too bad. I'm aware of its daily basis but it doesn't bother me or restrict me. If was diagnosed with lung cancer it would have knocked me over, but knowing I have a fighting chance as things are, it's not bothering me. I worry about my children worrying so that's why I take care myself and it's a concern. 
Interviewer: if you were given a chance to take part in an exercise programme would you be keen to take part in it?

Patient: ya, I'm keen to put in anything I can. There could be something which benefits me personally.

Interviewer: are there any particular exercises that you find beneficial that you are keen on doing?

Patient: no, I've never been a keep fit for nothing sort. I've been playing football and all that. I've always done walking and being active. I've had a garden, a house, kids, dogs, so I've always been busy you know. I don't need to go to the gym.

Interviewer: you talked about the physical benefits of exercise, do you think there are any other benefits of exercise related to you condition?

Patient: I don't know. I think it might be beneficial to the upper body. I can't lift things much these days. I've sometimes found lifting whatever across the garden these days, but before it was easy. So that side of it may.

Interviewer: form you're prospective what do you think encourage people with IPF to do exercise?

Patient: when I see some people in the support group I think, you know you are much younger to me but look older to me and I think if it is the condition or the life style prior to the condition. Maybe they have been diagnosed as the same point as I was and did, they thought that's it. And when I had that shortness of breath in February it was quite depressing. So, if they are feeling like that there is no going back for them. If they were diagnosed at the same point as I was, much encouragement should be given to exercise and maybe role models are what they need. The exercise wouldn't stop the disease, but it might slow it up. (The rest of the conversation was deleted because it was irrelevant to the question). When I went there $1^{\text {st }}$ it was depressing maybe it was bad timing with things with my wife and all.

Interviewer: apart from exercise and support group do you think there are any other elements to be added to a rehab programme?

Patient: I don't really know. (The rest of the conversation was deleted due to irrelevance to the topic). The other thing is when you tell people about an exercise programme, they say this and that, but if it was a walking programme it might be more attractive to some. The fact that you are taking the to a gym environment it might put them off.

Interviewer: how does your family feel about you going on to do an exercise programme?

Patient: they will be like get on with it. They would say l'm an inspiration to them. I don't think anyone would object it. (The rest of the conversation was not included because it was irrelevant to the question).

Interviewer: that's amazing. 


\section{PATIENT INTERVIEW TRANSCRIPTION}

(patient 6)

Age: 73

Interview date: 19.07.2016

Location: Swansea University Bay Campus

Conducted by: Dr. Joan Hudson and Mr. Sameera Senanayake

\section{START OF THE TRANSCRIPT}

Interviewer: thank you very much for coming today. Could you tell me a little bit about IPF and how it was diagnosed?

Patient: Yes, diagnosed in 2006, purely by chance. I had a cough and had two sets of antibiotics and it didn't get shifted as all. So, I went back to the doctor and he said you have a lung problem, you should go for an x-ray and see a chest consultant. Then I met Dr. Harrison and he said that I have IPF, which I've never heard of. $1^{\text {st }}$ thing which came in to my mind was lung cancer because I was a smoker but after having $x$-rays and biopsy it was confirmed what it was. And I managed it very successfully until March. Up until then I went to the gym twice a week and I was not on any medication, I'm still not on any medication but tomorrow Dr. Harrison is going to put me on some tablet.

Interviewer: OK so, you were going to the gym a couple of times a week and what sort of things were you doing there?

Patient: the bike, treadmill and occasionally using weights.

Interviewer: when did you stopped smoking

Patient: 2005

Interviewer: so, the exercise was helping you to manage your condition

Patient: yes definitely, I stopped going when I was ill, and I haven't been back since. I took part in the pulmonary rehabilitation programme in Moriston for six weeks and from there I got referred to another local council.

Interviewer: so, you stopped doing exercises on your own

Patient: I used to go for walks, but I haven't got energy to do it anymore

Interviewer: how far would you walk typically? 
Patient: I walk down the marina a couple of times to get coffee. I walk the dog twice a day and I had no problem. But since March its really knock me.

Interviewer: has it affected any other aspects of your life?

Patient: I've lost some weight and I can't seem to put it back on, that seems a problem.

Interviewer: when you were doing exercise what kind of benefits was it giving you?

Patient: it was keeping my lungs clear but now I have lot of mucus and flem. And it felt better. When I was done you felt that you have achieved something.

Interviewer: so, when you said it made you feel better, did it make you feel better about having IPF?

Patient: IPF is a disease I can't do anything about. All I can do it is to prolong it as much as I can. And whatever I can do to do that I will. I know a few people that are going to the rehabilitation course and they complain about being depressed. Well I'm not depressed, vie accepted what I have got. And try to be positive.

Interviewer: Did you feel like that from right at the start?

Patient: oh yes. I mean I possibly didn't realise how serious it was, but I got lot of knowledge over the years.

Interviewer: so, when you were $1^{\text {st }}$ diagnosed, what kind of information did you get?

Patient: I got information from Dr. Harrison and the rest from the internet. Since we started the group, I realize so many people in the area had the same problem. We talk about how it affects different people in different ways and for me I find it difficult to walk when it is windy and when it's hot, wet and cold it affects you. It seems whatever the weather condition is it affects you. You feel lethargic, you have no energy and you cough. It's not an easy disease to get along with.

Interviewer: so how do you get along with it?

Patient: I carry on how I am you know, I except what I have got, and I adjust in to it.

Interviewer: So, you said you built up information as you went. What sort of information would have been useful at the start when you were diagnosed? Not just information, what else could have been offered to you?

Patient: I think there is more awareness now, I don't think there was a great deal available at the time. So, I can't see it that way. As I said I was surprised to see how many people got the problem.

Interviewer: so, when you meet with other people, what's it like at the support group? 
Patient: we have a cup of tea; cake and we talk about how things are. They get people from different ways of life to talk about the problem. Rest of the conversation was deleted because it involved a political figure and is not relevant). There was a lady from wales health, and she talked about the problem and what is happening. We had a talk about what benefits we are entitled to. It's just people getting to gather and talk.

Interviewer: what do you think the biggest part?

Patient: just having a chat about families and how they are coping, you know that's the biggest part of it. Getting to gather and talking to people with the same problem

Interviewer: so, it's a positive thing?

Patient: Oh yes.

Interviewer: do you experience any negative result?

Patient: No, not really. I'm a half glass full person.

\section{Interviewer: what kind of industries have you worked in the past?}

Patient: I worked in the steel industry. I worked in templates section. I had to go to the plant quite a bit, talk to people out there so if that had to do with anything I don't know. I used to work in an old building with asbestos walls, sometimes when you move a chair to the wall and the asbestos sheet cracks and if it's a part of the problem, I don't know. The house I am living in has plaster ceilings and it had black motor (type of a dust) and I don't know if it caused the problem.

\section{Interviewer: going back to the rehab programme, how did you find that?}

Patient: very good. It was on Tuesday and Friday, $1^{\text {st }}$ hour was exercises and the second hour was a cup of coffee and talk about the illness.

Interviewer: the exercises you did, was its weight based, was it standing, seated?

Patient: there was a treadmill, a rowing machine and a couple of bicycles which I didn't like. The weights the medicine ball things, the stretch band, leg weights, so there was plenty there. But you did what you wanted alternating between arms and legs.

\section{Interviewer: what did you like about it?}

Patient: again, it was the people with the same problem, finding about how they coped. (Deleted from the transcript because the patient was talking about another patient in the rehabilitation programme)

Interviewer: did you enjoy the exercise part of it? 
Patient: yes. Some of them were hard work. I mean the treadmill is fine, I was happy with that but it's hard to do with the oxygen on.

Interviewer: what kind of benefits did you feel from that?

Patient: it was nice to see people, it became a close gathering eventually. At the end we were missing each other. It was about talking the problem with the people who are aware about it. When I talk to people from work, they have no idea what they are talking about, because they don't have a clue.

Interviewer: so, you are looking forward to the next one?

Patient: yes.

Interviewer: what would encourage you to go to an exercise class?

Patient: I want to go back. To be honest o don't need encouragement. But I was going to a private gym, but I don't know if they would allow me to use oxygen because it is a private gym. But the local gym the trainers have been trained to handle oxygen and aware of it, so I feel safer.

\section{Interviewer: are there any exercises that you would prefer to do?}

Patient: the treadmill. I walk uphill on it. I go on it for 25 minutes and I go on weights for 25 minutes and that will be it then.

Interviewer: there are some people in your situation that don't want to do exercises. What do you think that will encourage them to start doing exercises?

Patient: I think lot of them have depression. I don't know if it is the medication or how they are. They have a different look of life.

Interviewer: what does have IPF mean to you?

Patient: I accept the illness. I can't avoid the end of it because I know what is coming. But I make the most of what I got with whatever the time I have got left. It's a condition which affect all ages and areas of life. (Rest of the conversation was removed because the patient was talking about other patients involved in the rehabilitation programme).

Interviewer: what does your family think about your diagnosis?

Patient: my daughter worries, but my son had a shock when he saw me with the oxygen. He didn't realize how bad it was. (Rest of the conversation was deleted because the patient was talking about personal matters relating to his son).

Interviewer: are they supportive of you being active and doing exercise? 
Patient: I got told off by my daughter cutting the grass. But I got to do it. I got to do it when I get the chance.

Interviewer: what do they think about the exercise classes?

Patient: they think it's quite good. And the grandchildren care a lot. They encourage me to go. I think it does me good.

Interviewer: is there anything you would like to add?

Patient: I think when I was diagnosed little was known about the illness. So, it's only in the past 5-6 years that they have known more about it. If I can help in anyways, I try to do it.

\section{END OF THE TRANSCRIPT}




\section{PATIENT INTERVIEW TRANSCRIPTION}

(patient 7)

Age: 53

Interview date: 19.07.2016

Location: Swansea University Bay Campus

Conducted by: Dr. Joan Hudson and Mr. Sameera Senanayake

\section{START OF THE TRANSCRIPT}

Interviewer: $\mathbf{1}^{\text {st }}$ thing which will be really useful for us is to get an idea of the context of IPF, when you were $1^{\text {st }}$ diagnosed with IPF.

Patient: First I was having all this trouble last year and I had a medical problem from that I went to get sort it out and they took a scan and dealt with it. The doctor said besides that "I had a look at your lungs and I'm not happy with it and I'd like to refer you", and I thought nothing of it to be honest, I thought ya fine no problem.

Two months down the line I got referred to a guy called Dr. Shetty. He gave me a few tests to take and said he thinks it IPF, but we need to diagnose it to see what it was. So, I had a biopsy in January or February, and it came out to be what they thought it was. He said because of my age I could be a good candidate for a new set of lungs, but he had to take it to the committee and put my name forward. So, at the moment I'm in limbo. There is this new medication which stops your lungs repairing itself, but you have to be in a certain percentage. At the moment I'm in the good side rather than the bad side and what will happen is, when I drop to the bad side, I start taking the medication and that's when I will be there and the next line. And if I don't get a lung transferred at that point and they will say it's too late and that's where I am at the moment. So, flight of stairs panting like a bulldog. Anything uphill panting like a bulldog. It's horrible. If I bend down quickly, I feel dizzy. It's been really restrictive what I want to do in the house, garden, if I carry anything heavy it's too much, things like that. It's a nightmare.

\section{Interviewer: so, it's quite resent for you?}

Patient: ya, quite resent. After operation I went back to work, and they made major changes to my job to make it easier for me. It been really good work wise, they encourage me to work because if you push the lung its better because if you sit and work your lungs will go bad faster. So that's the position I'm in now.

\section{Interviewer: what do you do for work?}

Patient: I'm a refuse carrier and walking 15-20 miles a day picking up rubbish, I couldn't do that anymore. So, they put me on to sweeping side where they gave me a van where I go around the shops and do the bins. That what I do which is a lot easier now. So, I'm walking 20-30 yards maximum back in the van go to the next one. 
Interviewer: is that what you have always done?

Patient: I have done that for the last 25 years. Before that I was delivering bread and before that I worked for Sainsbury.

Interviewer: was that doing manual work?

Patient: yes.

Interviewer: in a factory?

Patient: in a supermarket running produce and frozen foods.

Interviewer: have you ever been a smoker?

Patient: was a smoker, a 20 man a day up till the age of 26 . As soon as we had our $1^{\text {st }}$ daughter I stopped. Don't drink don't smoke.

Interviewer: How old are you

Patient: I am 53 now.

Interviewer: being diagnose with IPF last year, how did you feel about that?

Patient: pretty depressing, I've got to be honest, l've look it up on the internet got some scary bits and told my wife there are some scary things out there that I don't want to know about, so if we are going anywhere you are going to come with me and when they we start talking about horrible things I'm gona get up and walk out because I don't want to be worrying about it. So, she has taken all of that. I think I've got 5 years if I don't get a set of new lungs and on medication it may extend it to ten years. But when it going to go to that stage and have to carry a bottle around like that I don't know. It's pretty scary. Because what I was doing, with all the rubbish I was picking up, I thought ill blame the council, and when they did the biopsy, they didn't find anything. Now they have all the health and safety rules, totally different ballgame. Couple of years ago I was picking up stuff where people come and dump thigs off vans and it was building stuff like plater board, so I think that could have caused it as well. But I can't prove it, so I have no case against the council.

\section{Interviewer: it a difficult condition isn't it}

Patient. Yes, sitting here I look fine. They say there is nothing wrong with you, try walking flight of stairs it's really horrible.

Interviewer: what kind of information did you get? Did you get right information, lot of information? 
Patient: No, my wife saw what the doctor was writing, and she got some letters off it and looked it up $1^{\text {st }}$ to see what we were getting in to, and it's nasty. There are things that I don't wana know down the line. She knows I don't, I would rather keep it that way.

Interviewer: sounds like she is great support.

Patient ya, she is. Really good support.

Interviewer: you also mentioned some advice that you got about keeping the lungs moving

Patient: they offered me a course at Morriston for 7 weeks where you go twice a week for an hour, basically it was doing different exercises. I thought with the work I do I don't need to do exercises. I got a dog and I walk with him up and down a canal and get quite a distance. At the end of that term my improvement worked out to be $33 \%$ in my lung capacity. I was doing rowing machine, steps, treadmill and I was really getting breathless, but I went really hard. May be there is something in it.

Interviewer: what type of exercise would you like to do?

Patient: I could probably do biking, walking, running is out, that's about it actually, but if I am going out, I need to plan a route which I know is flat. I want to get somewhere I want to get back and if I can't get back, I want to call somebody to ask to come and pick me up. I feel like an old man in 90. I think what's going on here, it's not right you know

Interviewer: given this diagnosis, what does it mean to you to be diagnosed with IPF?

Patient: I feel picked on, I feel I haven't smoked for all these years, I don't drink, I'm not overly fat, why is this happening to me? There are lot of people who are older than me who haven't got it. My father died when I was very young, so I don't know if he had it, my mother didn't have it, if it a trait in the family, I don't know. My brother is OK, so it looks like I'm the $1^{\text {st }}$ to have it in the family. It's not good but you would have to get on with it.

Interviewer: obviously there are lot of negatives associated with the conditions, do you see any positives?

Patient: positives would be my age, I'm a good candidate for a lung transplant.

Interviewer: are there any kind of stipulations about your suitability

Patient: No. It's just a case of how bad it gets. At the moment I'm too fit to have them, it wouldn't benefit me. It's a fine line. If you go below where you are not fit enough to handle it, they won't do it either. That where I don't want to get to. That's my main fear.

Interviewer: going back to the exercise programme you did, you said it improved your lung function. Were there any other benefits from it? 
Patient: I'm sleeping better on the days I was doing it. Social thing was OK, it's nice to say, it's a horrible thing to say, but it was nice to see there were people worse than I was. Because lot of people were carrying the bottles around and they had tubes up their nose and they were hardly doing anything. And I told myself I am not that bad yet. That was a bonus. I also had few good discussion about what happens, what you get, because there are so many different varieties of it. And I learnt few things like when it comes to climbing steps it's easier to breathe in when u take off the step and breath out when you land on the step. Ya little things like that.

Interviewer: would there be more things like that, which would be useful do you think?

Patient: don't know

Interviewer: What types of exercises do you think that will be useful in an organised programme?

Patient: doing weight was quite useful because that's all breathing as well. Treadmill was OK, because you could go on speeds you want to go. Rowing was a bit hard because it crunches and make it hard to breath.

Interviewer: you mentioned things that are good to do and not good to do, and the reasons, are there any others like rowing?

Patient: rowing is not good, we did cross trainers and it was a killer because you use so many limbs at the same time. I tried doing that and in 3-4 minutes I was done for the day.

Interviewer: are there any others that are useful? Any daily activities?

Patient: bending thangs up is a problems, carrying things is a problem, heavy weights area a problem.

Interviewer: what do you think motivates people to go and take part in exercise training programmes?

Patient: if thy carried on what I was doing with this twice a week I would have carried on doing it. It was disappointing that it ran for a limited time. Because you met people and after that you got a cup of tea sit down and had a chat. It was nice. But you won't get that in a gym.

Interviewer: does your wife encourage or discourage you to exercise?

Patient: she can go both ways. If she sees me doing something I shouldn't do, she would ask the girls to do it. Other than that, if I say I need to do it she would let me. If I go up the stairs and I forget something she would ask the girls to do it and I would say I will want to do it because I need to do it. Some. Eventually she is talking about us moving downstairs and stay downstairs once it gets to a certain point, well I don't want that. I'd rather take 10 minutes to go upstairs to go to bed than staying down stairs all the time. That's just me 
Interviewer: so, you are trying to stay active in your day to day life style?

Patient: ya, I'm not pushing it too much, but I want to push it to a point where you start getting breathless.

Interviewer: about the exercise programme, are there any other important elements that you think relevant?

Patient: there were lot of attitude, mentally how you look at it. Don't look at it half empty and but half full. I suppose in a way that does help.

\section{Interviewer: was that a structured session?}

Patient: no, it's just patients talking while exercising. Its informal thing.

Interviewer: is there any other thing that you would add to it?

Patient: I would make it a bit longer. The individual session. Because there was a warmup and you would lose 20 minutes and it was a lot of time lost. I like to see that extended for a couple of hours.

Interviewer: is there anything more to add?

Patient: no, I don't think so. There are not lot of information out there and there are many varieties of it. So, what's good for me might not be good for someone else. And that's a big things. And what information out there it's scary. They say what you have and what will happen in the end and that's it. There is no information on what will happen in between.

Interviewer: if you were to tailor how information would look like, is there a better way to present it?

Patient: I think you need it in age groups, which would help. When I went to the doctor he said if you were 20 years older, I would say you can't do anything, but since you are such a young age you have options. So, you need it in age groups. If you have that my route will be there to there to there and in 10 years it will be there to there.

Interviewer: thank you for taking part in the study.

END OF THE TRANSCRIPT 


\section{PATIENT INTERVIEW TRANSCRIPTION}

(patient 8)

Age: 73

Interview date: 26.07 .2016

Location: Swansea University Bay Campus

Conducted by: Dr. Joan Hudson and Mr. Sameera Senanayake

\section{START OF THE TRANSCRIPT}

\section{Interviewer: when did you get your diagnosis on IPF?}

Patient: it was about 12 months ago now, very shocked, nearly upset at the time. Coming to terms with it slowly but that's life. I was please it wasn't cancer. Because I wasn't able to stop coughing at that time.

\section{Interviewer: so, the shock was about your life style?}

Patient: well no, I just had this cough, it went on and on, so I went to the doctor. I also get this pain in my chest. She sends me for an asthma test, and it was negative. Then a heart scan and it was OK. And I was still coughing I thought I have cancer. Then she sent me to Dr Harrison, and I had all these tests. After that I met his registrar. He went in met Dr. Harrison and he came out and I though oh god its cancer and he said you have got a lung disorder. But I want to discuss it with my colleagues before I diagnose what it is. And after my holidays I came back, and he said you have fibrosis in the lung. I though thank god it's not cancer, but I was upset at the same time. They gave me a booklet and I wish I didn't read it. It said I would life for 3 years, but I've since heard that people live for 7-10 years and I calm down a bit. That came as a great shock. I used to be a smoker, but they said that day don't know that smoking caused it. (Rest of the answer was removed because it wasn't relent to the topic)

\section{Interviewer: what do you do for living?}

Patient: I've done all sort of things. I was a supervisor at CNA's for a year. I was on the management programme. Then I worked in a solicitor's office, then I was a receptionist at a doctors before I retired.

Interviewer: but no kind of heavy industry?

Patient: no, never, nothing like that. I've had a stressful life, don't know if that had anything to it.

Interviewer: at the time did you get enough information or right kind of information on IPF?

Patient: to give it to someone as soon as diagnosed it, I think it was not a good idea. I read it and saw three years I though oh my god. Then my daughter read it and she was badly distress and she kept it from me. When it is sitting there in front you think OMG.

Interviewer: you said you have come to terms with it.

Patient yes, I have started to come in terms with it. These days I think oh god what is going to happen to me, am I going to end up in a nursing home or dead and my daughter says you will never go in to a nursing home but you know these days you can't say that, you never know what's going to happen. My husband is not well too. And if I get up thing I get more out of breath.

Interviewer: have you got any way of coping it? 
Patient: well to be honest I carry on and tell myself I live another 20 years and I get on with it. That's the only way I could cope with it.

Interviewer: how old are you? Patient: 73

Interviewer: on a day to day basis how does your condition affect you?

Patient: I find it very difficult going upstairs, I get out of breath. I'm overweight and have knee replacement and that doesn't help. But my draught helps me change the beds because I find that difficult. And if I walk a lot I get out of breath. But other than that, I go out and have a nice time. I just came back from Spain last month and I was a different, the weather was so nice and the moment I came back it was so bad. I was veezing and it was so bad. The weather makes a difference without a doubt.

Interviewer: Although you get out of breath, do you do what you normally do?

Patient: Oh no. I used to play golf and I can't do that anymore. I can't dance. I play bridge a lot and that's my saviour. Other things I manage to do. Like walking the dog on the flat.

Interviewer: how often do you walk the dog?

Patient: I don't do it mush these days because they are with my daughter, but when we have the dogs it at least three time a week.

Interviewer: does anybody in the hospital talked about any exercise or rehab programme?

Patient: no. I've been on the rehab programme. I enjoyed it. I thought it was good. (The rest of the conversation was removed because she was talking about other patients). I think if you are going somewhere (rehabilitation programme) the people you are going to should know about the illness and should know how far you could go. I'm waiting to go to one of the programmes and I don't know what it's like. The nurses who is sorting this out should check what they do before they push you out. But I think it was good, the one in the hospital.

Interviewer: what was good about the hospital one?

Patient: I felt safe. There were people there watching you and when you had enough you could stop. There was company, people with the same disease. I found I was looking forward to going. And I thought it was doing me good.

Interviewer: did it help you physically?

Patient: ya it was. Can't put a finger on it, but I feel better in myself. I also lost half a stone.

Interviewer: did you feel any better in any other way?

Patient: not really no. talks afterwards, I found it stressful. I didn't find it useful because I wasn't able to cope with that, the stress I have gone through. The breathing exercise was good. There was a lot of good. The actual gym I enjoyed.

\section{Interviewer: dis you find the exercises they had useful?}

Patient: yes. They had the walking machine, the bike. They had starch bands. And I found them help in some ways.

Interviewer: what type of exercise would you like to see in the programme in panarea? 
Patient: the bicycle, the walking machine. I like the elastic band. The medicine balls. Weights. I found them very helpful.

Interviewer: did you manage to do weight standing?

Patient: yes. (The rest of the conversation was removed due to irrelevance)

Interviewer: was there anything that you would add to it?

Patient: no, I don't think so.

Interviewer: what's it like to your family (the disease and exercise)?

Patient: my daughter knows, my husband knows. He had been very protective in a loving way. My son hasn't told him.

Interviewer: what does your husband and daughter think about you exercising?

Patient: they want me to do it. My husband thinks it's good. He wants to go on walks and then I worry about him because of his situation. But we do go for walks.

Interviewer: do you think the time duration of the individual workout session was enough.

Patient. Yes. May be 15 minutes more on the exercise part would have been better and cut down the talking session. And I would like to carry on the programme for more than six week.

Interviewer: what are your thoughts about the support group?

Patient: I like going there. They have people coming in and talk about the illness. Dr Harrison is there all the time. And it nice to chat with people with the same problem. When I see people with oxygen that what I dread. I don't know if I could cope with it. If you want to live you live but it's not me. (Rest of the conversation was deleted on patient's request).

Interviewer: what does have IPF mean to you?

Patient: Death. Certain definite death. That's what it means to me.

Interviewer: is there anything positive that come out of your diagnosis.

Patient: no, unfortunately no.

Interviewer: were you able to find information on IPF easily?

Patient: yes, I got that booklet. Even without that there are lot of thing in the internet.

Interviewer: thank you for taking part in the study. 


\section{PATIENT INTERVIEW TRANSCRIPTION}

(patient 9)

Age: 65

Interview date: 16.08 .2016

Location: Swansea University Bay Campus

Conducted by: Dr. Joan Hudson and Mr. Sameera Senanayake

\section{START OF THE TRANSCRIPT}

\section{Interviewer: When were you $1^{\text {st }}$ diagnosed with IPF?}

Patient: $1^{\text {st }}$ time I realized that something was wrong. I entered this cycling competition with my son that was a year ago. There were couple of young boys cycling around us. These boys accelerated and I thought I can do that and so I went couple of yards and I felt something was wrong. So, I took some rest on the pavement. My son told let's stop and I don't like giving in, so I walked rest of the way. I took some rest, had a banana and I was completely fine. And that was the beginning of it. (Rest of the conversation was deleted due to irrelevance).

Interviewer: so, it sounds like you were very fit back in the day?

Wife: you were when you were in the army because you had to do all those exercises and walk everywhere.

Patient: Yes. I used to walk on every Friday to see my girlfriend.

Wife: you haven't been too bad have you?

Patient: when I get it it's not very pleasant. I get breathless, can't talk properly, my voice goes for some strange reason and it's not very good. But I live

Wife: He rides the bicycle indoors so that's good. Sometimes he is not very good, but he is ok.

Interviewer: so everyday life hasn't changed a lot?

Wife: not really. He does get quite breathless. What it does really is that he can't walk too far. Also, his throat goes. I think there is a problem with his throat as well. Sometimes he can't speak. We went on a trip to Australia and new Zeeland and the before the trip he said he can't go. But after going to the doctor we decided to go. It affects him a lot, but it doesn't stop him doing what we want to do. So, it's been there for a long time, but he had been able to cope with it.

\section{Interviewer: so how have you coped with it for such a long time?}

Patient: I've been on the exercise bike; I've been walking slowly and doing gentle exercise. So, I have been taking care of myself.

Wife: he is taking care of himself, so he is controlling it by not straining himself too much.

Interviewer: is it because you had advised on it when you were diagnosed?

Patient: he is being seeing Dr. XXXXXX only for couple of years.

(Rest of the conversation was deleted from the conversation because it was irrelevant to the topic) 
Interviewer: When you met Dr. XXXXXX did you get a diagnosis on your breathlessness?

Patient: I saw a young doctor $1^{\text {st }}$ and she discovered my lungs were scared. Ten only I was diagnosed with IPF. I used to be a heavy smoker.

Interviewer: so, when you were diagnosed how did you feel about it?

Patient: it came as a little bit of a shock. It affected me but not in an excessive way.

Wife: sometimes you get very breathless and I notice when that happens, he is very tired. (Rest of the conversation was removed from the transcript because it was irrelevant to the topic.

Interviewer: if we were to offer you an exercise programme what would you like it to be?

Patient: as long as it's not strenuous I can do it. (Rest of the conversation was removed from the transcript because it was irrelevant to the topic)

Interviewer: So, what kind of exercises do you think you could do?

Patient: I can certainly go on the exercise bike, nothing hard but I find it useful for circulation on the legs.

Interviewer: do you share everything about your disease with each other?

Patient: we are pretty open about it and it been an excellent marriage. (Rest of the conversation was deleted from the transcript because it was irrelevant to the topic)

Interviewer: when you $\mathbf{1}^{\text {st }}$ found about your condition did you get enough information about it?

Patient: the impression that I got was that they haven't reached to a stage in the repair process of the disease and this is what's it's all about. They are trying to get more information.

Interviewer: have you got your own information about how might exercise help or not?

Patient: well, I find that the stretching is good, and the exercise bicycle is wonderful. We also go for walks around the lake. 
Interviewer: what is it about stretching that you find useful?

Patient: my intercostal muscles were very stiff, so I wasn't able to breathe properly. So, I stretch and eventually I get a comfort through that. And the more I did the better it became. (Rest of the conversation was deleted because it was irrelevant to the topic)

Interviewer: you mentioned about stretching. If you were asked to create a rehabilitation programme to manage your IPF, what would that look like?

Patient: starching is really good, and individual would be better because I can stop and start whenever I want, and it would help others too.

Interviewer: finally, we have technology everywhere. Do you think technology could help you with exercise?

Patient: no, it's not for us.

Interviewer: Thank you for taking part in our study.

END OF TRANSCRIPT 


\section{PATIENT INTERVIEW TRANSCRIPTION}

(patient 10)

Age: 60

Interview date: 25.08 .2016

Location: Swansea University Bay Campus

Conducted by: Dr. Joan Hudson and Mr. Sameera Senanayake

\section{START OF THE TRANSCRIPT}

\section{Interviewer: When you got your diagnosis what sort of information did you get?}

Patient: my background is in nickel and I have been exposed to obnoxious chemicals dust and so on. When I was $1^{\text {st }}$ diagnosed I had a persisting cough like most people, and it didn't clear for months. So, I went to see a consultant and they put me through a cat scan and that's where they picked it up.

Wife: but he had been having the cough two years before that.

Interviewer: how did you feel when you $1^{\text {st }}$ got your diagnosis?

Patient: Its ok. I didn't know anything about it, and I've been living for 10 years.

Wife: we found lot of information on the internet and it was a bit devastating because he is quite healthy. But we got on with life.

Patient: lot of people don't know what it is. They understand lung cancer but when it comes to IPF they are like what is that? (rest of the conversation was deleted because it was irrelevant to the topic.)

Patient: also, I've been on nine drugs which I didn't like at all.

Interviewer: Tell me little bit about the psychological effect of your diagnosis?

Patient: well, I wasn't happy on 9 tablets a day. It also getting grips with this condition. You have your ups and downs. It's a very debilitating condition. You have bursts of energy and bursts of you can't do anything. Simple things like taking out the bins, moving the lawn, cutting the bush such is very frustrating being used to carry out these things. But you have to accept it and carry on. It's very frustrating.

Interviewer: But you accept it? 
Patient: you have to. I mean the fatigue you get when you are doing these things, I feel like a battery, your energy levels go down and you would have to stop. With 6 grandchildren we had a busy summer. During the holidays they were with us and I wasn't able to involve with them as much as I liked.

Wife: I think this new tablet he is on has increased his energy levels and psychological effect is good.

Patient: but it's been a little stressful taking care of the kids. (rest of the conversation was removed because it was irrelevant to the topic).

Interviewer: did you find your stress made your condition worse?

Patient: not really. I don't like it when stress affects me.

Wife: we get on with it. Stress id part of life isn't it.

Patient: 40 years in the chemical industry l've had disasters and stress but at the end you accept it and move on with life.

Interviewer: it sounds like that how you have come to terms with IPF.

Patient: you have to try to make the best of life. That's the way the dice is rolled. I do get frustrated when its physical affects me, not being able to do things that I planned to do. You think about retirement and think it's nice not to go to work but the health doesn't keep up. You plan to do all these things that you wanted to do all these years. But here we are.

Wife: we did them as much as we could. We travelled a lot, but the past three years not so much.

Patient: I've also been on the rehabilitation programme at Morriston and its really good. You do half hour of exercise and the we had lectures on the condition, and it was really helpful.

Wife: the social side of it is really good. You share ideas and share how things are and we pass ideas.

Interviewer: so, it gives you a support mechanism?

Patient: yes, you get to see the different stages of the disease and lot of guys there use oxygen. I have oxygen but I only try to use it in an emergency.

Interviewer: how did you find the exercise part of the programme?

Patient: good. I've always exercised and I'm still a member of LC2. Next week we will start going back again. It benefits physically as well as mentally. 
Wife: we have friends coming in as well. So, it really good.

Interviewer: the mental and the physical benefits of your exercises, could you explain it a bit more?

Patient: I have always exercised. When this condition came along, you start to worry about if exercise good or bad for you. I found after exercising I slept better, woke up better and it improved my flexibility. Mentally you are feeling better too, and it helps. And with more exercise and steroids I'm desperately trying to put some weight on and improve my condition.

Interviewer: what kind of exercise do you do?

Patient: I can go on the walking machine. I can do three kilometres at $6 \mathrm{kmph}$ in 30 minutes. I do row for 10 minutes. Then I do pull ups with weights and some machine exercises. At the end I do spend about one hour there.

Wife: before the summer holidays there is a big improvement in his attitude. He is positive and I think it's the new drugs se is taking. During the winter I was worrying that he would go for depression. But it had made a big difference. (rest of the conversation was deleted due to irrelevance to the subject).

Interviewer: you said during the winter your mood was down. Do you think exercise helped you improve apart from drugs?

Patient: Oh yes, definitely. We will get back to it after the summer holidays.

Interviewer: going back on the exercise programme, you said you did a lot of walking. So, did you do the rest while seated?

Patient: most of it was seated. And most of them were strength once.

Interviewer: if you were to go on an advanced rehab programme, what should it look like to you?

Patient: I think the course at Morriston is good. I could cope very well. It didn't tax me that much.

Wife: we have always done a lot of walking.

Patient: I'm ok with flat but with angles it hard.

Interviewer: staying active, is it hard to do?

Patient: no. you just have to change yourself. We are going to start the gym again. 
Interviewer: if you were asked to exercise at home as a part of a rehabilitation programme. Is it something that you would follow?

Patient: They did it. I can't say I picked it up. It easier for me to jump in the car and go to the gym. If you are in the house it very distracting.

Interviewer: how important is it for you to have somebody with the knowledge to be there with you when you are exercising?

Patient: not too important. I know what I've got, and I choose exercised that I can do and can get away with.

Interviewer: do you think the individual session length of the exercise session at Morriston adequate?

Patient: I think half an hour is enough for most people in it.

Interviewer: Do you have anything else to add?

Patient: not really, I think we have said it all.

Interviewer: Thank you so much for both in taking part in the interview.

END OF TRANSCRIPT 


\section{Appendix C: Data accompanying Chapter 5}

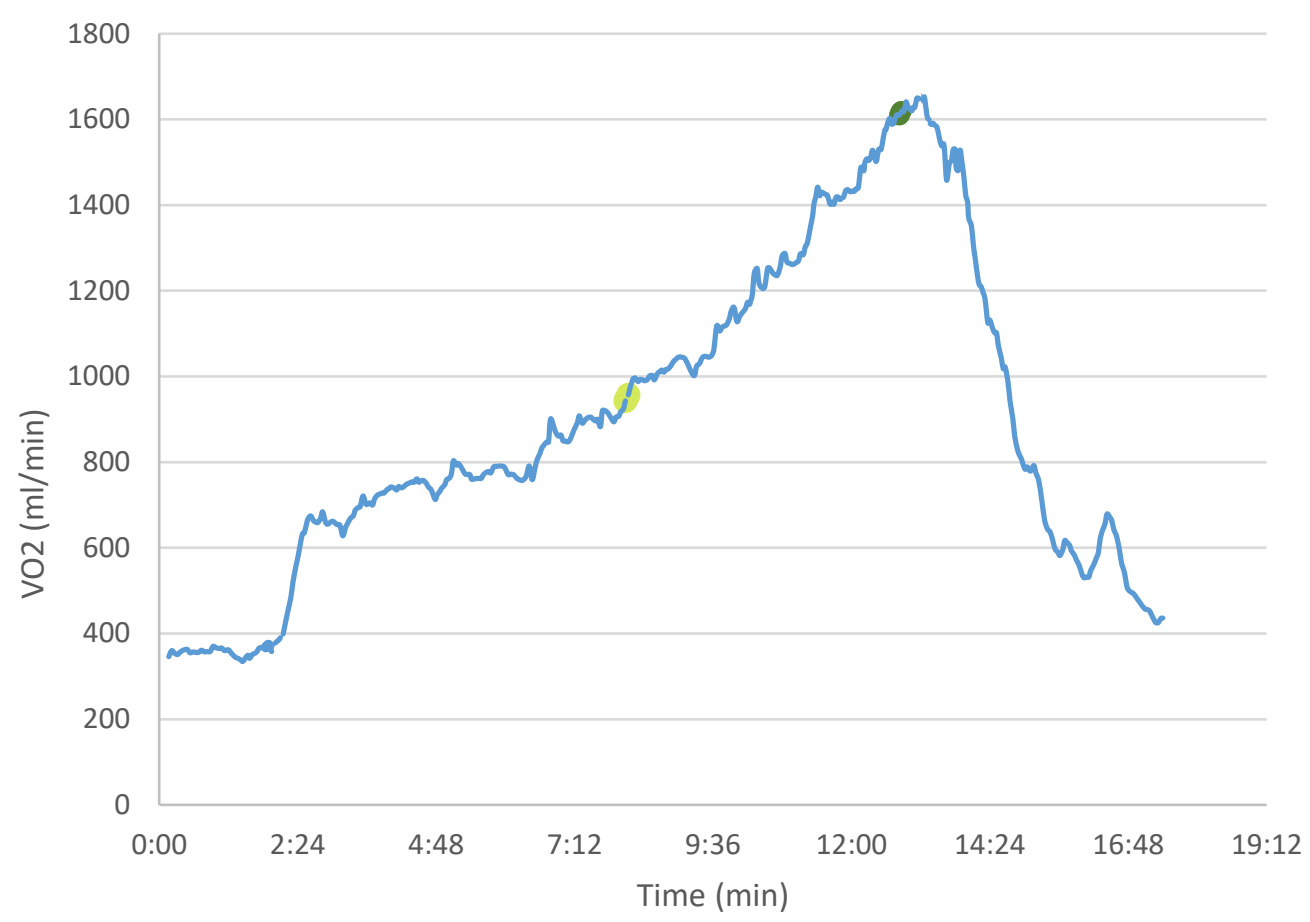

Figure C.1 Example plot of VO2 as a function of time for an individual patient (raw data). (Yellow dot indicates the Gas Exchange Threshold (GET) and green dot indicates $\mathrm{VO}_{2}$ max.)

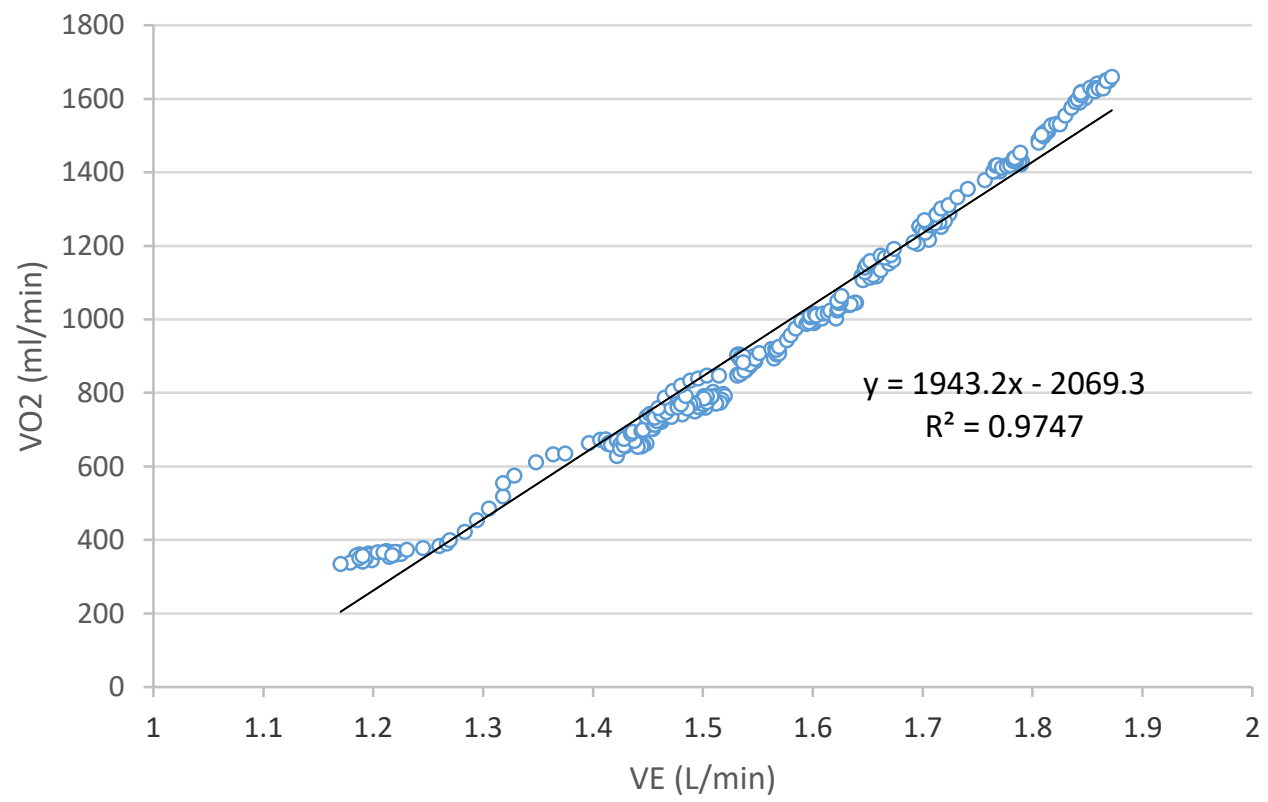

Figure C.2 Example plot of VO2 against VE, showing the calculation of OUES (slope of regression line). $Y$ axis represent $\mathrm{VO} 2$ and $x$ axis represent Log10 of minute ventilation (VE) 
Table C.3: Physiological responses to incremental (maximal effort) exercise tests pre- and postrehabilitation for each individual who took part in the study.

\begin{tabular}{|c|c|c|c|c|c|c|c|c|c|c|c|c|}
\hline & \multicolumn{2}{|c|}{ VE (BPTS) } & \multicolumn{2}{|c|}{$\begin{array}{c}\mathrm{VO2} \\
\left(\mathrm{ml} \cdot \mathrm{min}^{-1}\right)\end{array}$} & \multicolumn{2}{|c|}{$\begin{array}{c}\text { VO2 } \\
\left(\mathrm{ml} \cdot \mathrm{kg}^{-1} \cdot \mathrm{min}^{-1}\right)\end{array}$} & \multicolumn{2}{|c|}{$\begin{array}{l}\text { PEAK HR } \\
\text { (bpm) }\end{array}$} & \multicolumn{2}{|c|}{$\begin{array}{c}\text { PEAK } \\
\text { WORK } \\
\text { RATE (W) }\end{array}$} & \multicolumn{2}{|c|}{$\begin{array}{c}\text { TIME TO } \\
\text { PEAK WORK } \\
\text { RATE (min) }\end{array}$} \\
\hline $\begin{array}{l}\text { PATIENT } \\
\text { ID }\end{array}$ & Pre & Post & Pre & Post & $\begin{array}{l}\text { Pre- } \\
\text { PR }\end{array}$ & $\begin{array}{l}\text { Post- } \\
\text { PR }\end{array}$ & Pre & Post & Pre & Post & Pre & Post \\
\hline P1 & 74.8 & 79.1 & 1659 & 1639 & 14.9 & 15.2 & 124 & 136 & 108 & 109 & 13.11 & 13.12 \\
\hline P2 & 30.4 & 38 & 1040 & 898 & 10 & 8.4 & 73 & 75 & 29 & 59 & 11.16 & 6.12 \\
\hline P3 & 92.3 & 80.8 & 1590 & 1538 & 18.7 & 17.1 & 142 & 125 & 100 & 86 & 12.34 & 13.12 \\
\hline P4 & 49.1 & 47.4 & 1113 & 1079 & 13.1 & 12.8 & 138 & 135 & 55 & 62 & 12.07 & 12.59 \\
\hline P5 & 64.7 & 59.7 & 1269 & 1538 & 14.6 & 13.5 & 94 & 88 & 81 & 65 & 12.22 & 13.58 \\
\hline P6 & 64.9 & 43.1 & 1605 & 1119 & 16 & 12.5 & 147 & 127 & 99 & 72 & 15.05 & 10.55 \\
\hline P7 & 86.8 & 90.5 & 1809 & 1912 & 18.3 & 19.3 & 141 & 111 & 158 & 128 & 13.03 & 14.07 \\
\hline P8 & 60.5 & 35.2 & 1321 & 834 & 15.1 & 9.6 & 99 & 75 & 93 & 49 & 12.29 & 9.02 \\
\hline P9 & 52.6 & 53.5 & 1057 & 916 & 14.3 & 12.8 & 101 & 56 & 94 & 35 & 9.2 & 7.38 \\
\hline P10 & 75.3 & 70.5 & 1600 & 1467 & 28.4 & 26.2 & 159 & 100 & 148 & 97 & 16.39 & 16.35 \\
\hline P11 & 43 & 58.6 & 1168 & 1382 & 12.5 & 15.4 & 127 & 72 & 135 & 96 & 10.51 & 14.48 \\
\hline P12 & 34.7 & 48.2 & 1138 & 1256 & 14 & 18.2 & 126 & 65 & 132 & 83 & 12.27 & 11.14 \\
\hline P13 & 22.4 & 30.7 & 849 & 1082 & 9.5 & 16.9 & 93 & 46 & 83 & 50 & 7.29 & 7.1 \\
\hline P14 & 36 & 63.3 & 1073 & 1282 & 14.3 & 17.1 & 115 & 76 & 129 & 99 & 10.43 & 11.47 \\
\hline P15 & 53 & 72.3 & 1194 & 1313 & 13.9 & 15.3 & 116 & 66 & 125 & 72 & 9.56 & 10.49 \\
\hline
\end{tabular}


Table C.4: OUES for pre- and post- PR states. ( $R^{2}$ : Coefficient of determination.)

\begin{tabular}{c|cccc}
\multicolumn{1}{c}{ PRE-PR } & \multicolumn{2}{c}{ POST-PR } \\
\hline PATIENT ID & $\begin{array}{c}\text { OUES } \\
\left(\mathrm{ml} \cdot \mathrm{min}^{-1} \cdot \mathrm{L}^{-1}\right)\end{array}$ & $\mathbf{R}^{\mathbf{2}}$ & $\begin{array}{c}\text { OUES } \\
\left(\mathrm{ml} \cdot \mathrm{min}^{-1} \cdot \mathrm{L}^{-1}\right)\end{array}$ & $\mathbf{R}^{\mathbf{2}}$ \\
P1 & 1943.2 & 0.97 & 1754.1 & 0.97 \\
P2 & 1467.2 & 0.85 & 1121.2 & 0.84 \\
P3 & 1466.6 & 0.98 & 1399.2 & 0.96 \\
P4 & 1274.9 & 0.98 & 1245.8 & 0.98 \\
P5 & 1321.2 & 0.97 & 1750.6 & 0.98 \\
P6 & 1828.7 & 0.98 & 1889.4 & 0.96 \\
P7 & 1688.9 & 0.98 & 1929.6 & 0.98 \\
P8 & 1426.0 & 0.99 & 1185.1 & 0.98 \\
P9 & 1703.6 & 0.91 & 1684.9 & 0.93 \\
P10 & 1731.3 & 0.98 & 1664.8 & 0.98 \\
P11 & 1886.1 & 0.96 & 1570.6 & 0.97 \\
P12 & 1509.2 & 0.97 & 1348.7 & 0.97 \\
P13 & 1377.2 & 0.85 & 1423.4 & 0.90 \\
P14 & 1538.5 & 0.94 & 1292.0 & 0.98 \\
P15 & 1378.8 & 0.98 & 1374.7 & 0.98 \\
Mean & 1569.4 & 0.95 & 1508.9 & 0.95 \\
SD & 205.5 & 0.04 & 249.5 & 0.04 \\
SEM & 53.1 & 0.01 & 64.4 & 0.01 \\
\hline
\end{tabular}


Table C.5: Acceleration Capacity and Deceleration Capacity indices for each individual participant pre and post PR. ( $\Delta$ Post-Pre $=$ absolute change following PR, $\Delta(\%)=$ relative change following PR).

\begin{tabular}{|c|c|c|c|c|c|c|c|c|}
\hline PATIENT ID & & ELERATI & Capacit & & & ELERATIC & Capaci & \\
\hline & Pre-PR & Post-PR & $\begin{array}{c}\Delta \text { Post- } \\
\text { Pre }\end{array}$ & $\Delta(\%)$ & Pre-PR & Post-PR & $\begin{array}{c}\Delta \text { Post- } \\
\text { Pre }\end{array}$ & $\Delta(\%)$ \\
\hline 1 & -18.1 & -19.2 & 1.0 & 6.0 & 15.4 & 13.2 & -2.2 & -14.7 \\
\hline 2 & -3.9 & -5.1 & 1.2 & 30.1 & 5.1 & 4.6 & -0.5 & -11 \\
\hline 3 & -3.9 & -3.9 & 0 & 0 & 3.1 & 3.1 & 0 & 0 \\
\hline 4 & -1.4 & -3.4 & 2.0 & 144.6 & 1.4 & 3.3 & 1.9 & 138.5 \\
\hline 5 & -2.2 & -5.6 & 3.3 & 151.1 & 2.6 & 5.3 & 2.6 & 101.4 \\
\hline 6 & -2.7 & -1.2 & -1.4 & -52.8 & 2.1 & 1.3 & -0.8 & -38.3 \\
\hline 7 & -1.1 & -2.7 & 1.5 & 138.1 & 1.2 & 2.9 & 1.6 & 127.1 \\
\hline 8 & -16.5 & -15.8 & -0.6 & -4.2 & 17.2 & 18.2 & 0.9 & 5.8 \\
\hline 9 & -1.8 & -2.2 & 0.4 & 22.5 & 2.0 & 2.4 & 0.4 & 21.6 \\
\hline 10 & -3.3 & -2.9 & -0.4 & -12.4 & 3.7 & 2.4 & -1.3 & -34.8 \\
\hline 11 & -1.2 & -1.3 & 0.0 & 6.8 & 1.3 & 1.2 & -0.1 & -7.6 \\
\hline 12 & -1.7 & -2.4 & 0.6 & 36.6 & 1.7 & 1.7 & 0.0 & 3.7 \\
\hline 13 & -2.2 & -3.2 & 0.9 & 42.5 & 2.0 & 3.2 & 1.1 & 54.5 \\
\hline 14 & -2.7 & -2.5 & -0.2 & -7.1 & 2.3 & 2.6 & 0.3 & 15.4 \\
\hline 15 & -5.7 & -4.0 & -1.6 & -29.1 & 5.6 & 3.3 & -2.2 & -39.9 \\
\hline
\end{tabular}




\section{Appendix D: Data accompanying Chapter 6}

Figure D.1: Modelling of individual participants' temporal $\mathrm{VO}_{2}$ data for (a) pre-PR and (b) post-PR conditions

(a) Pre-PR

\section{Patient 14}

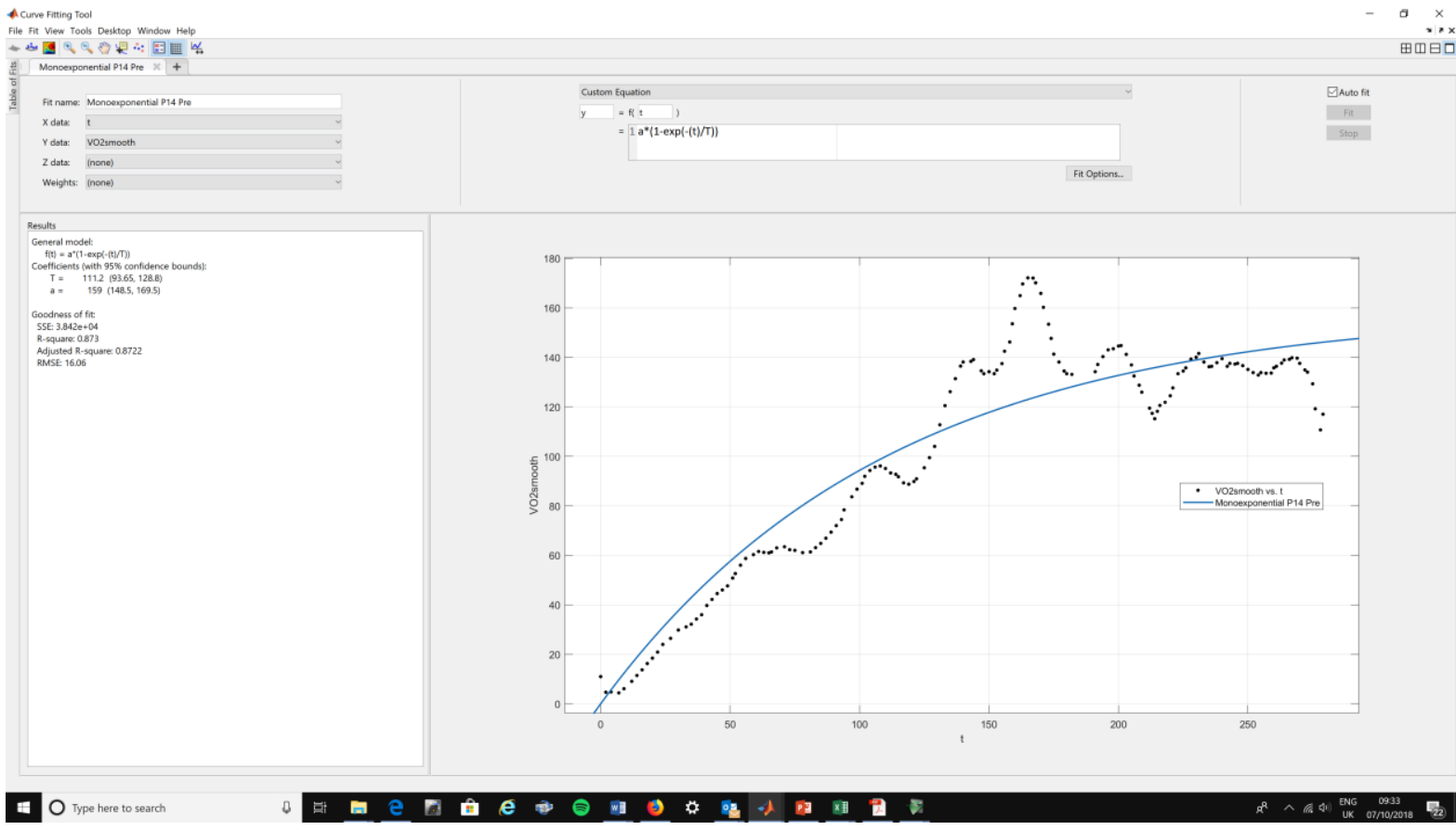

Patient 13

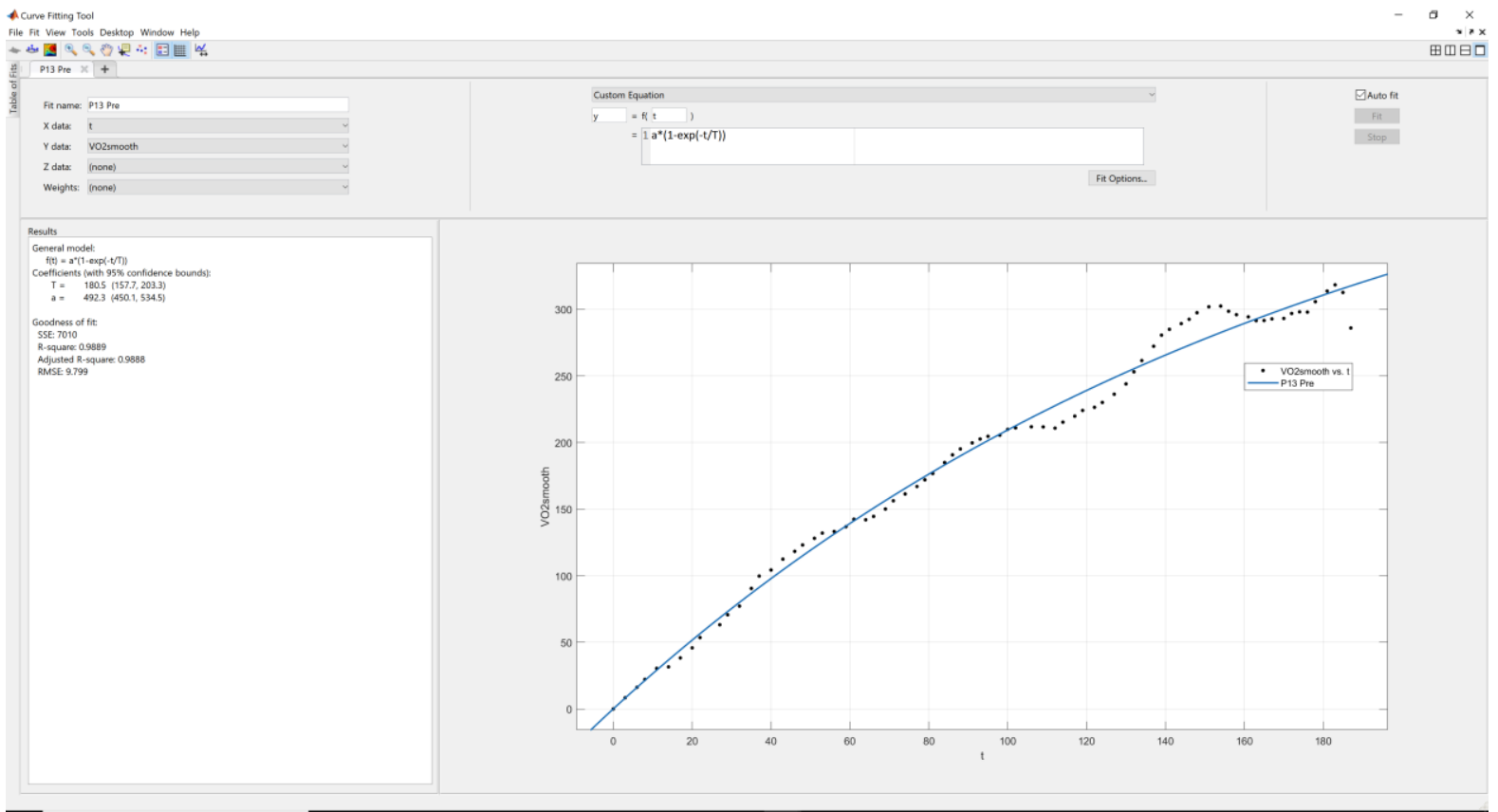


Patient 12

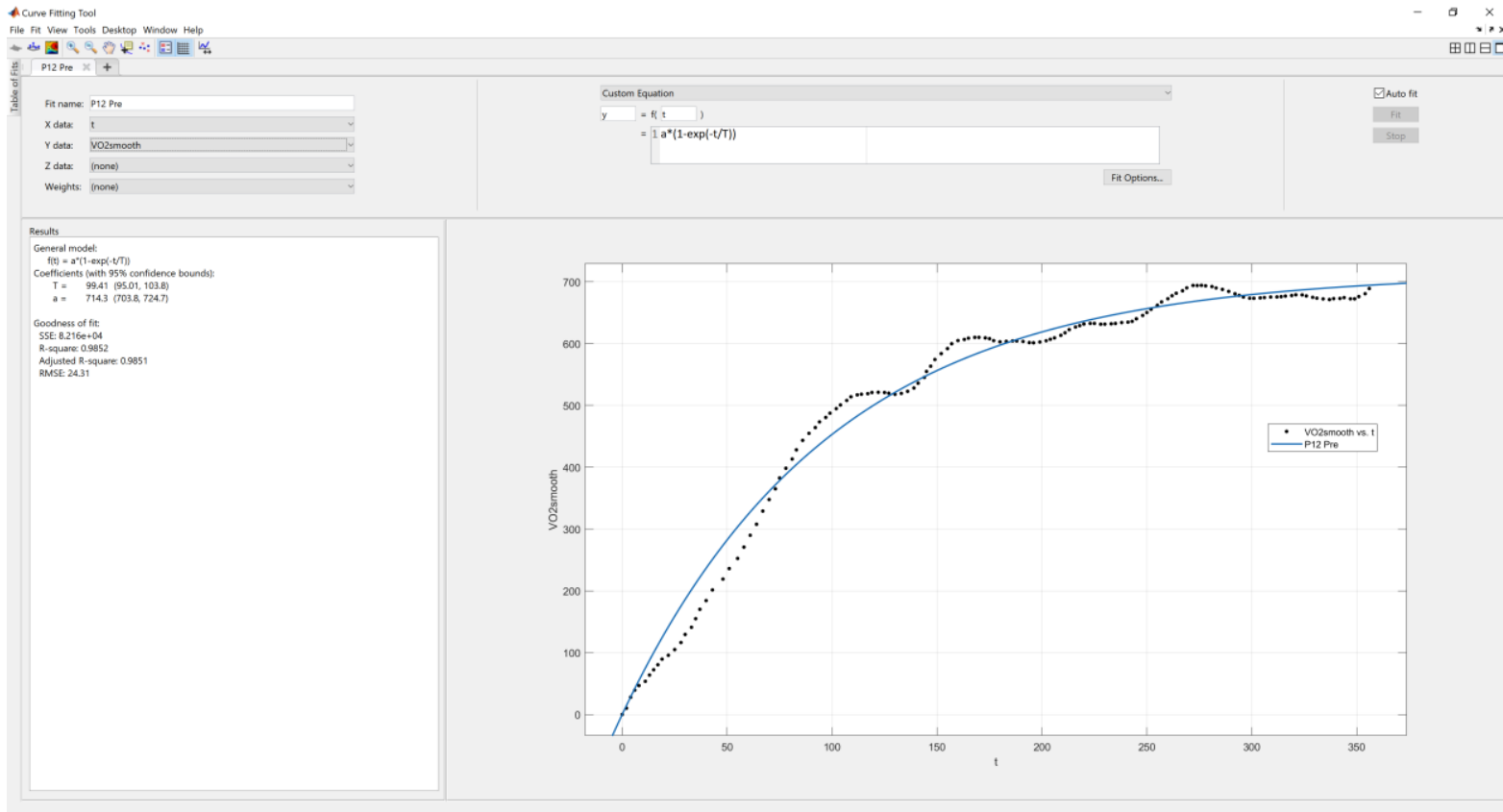

E O Type here to search

Patient 11

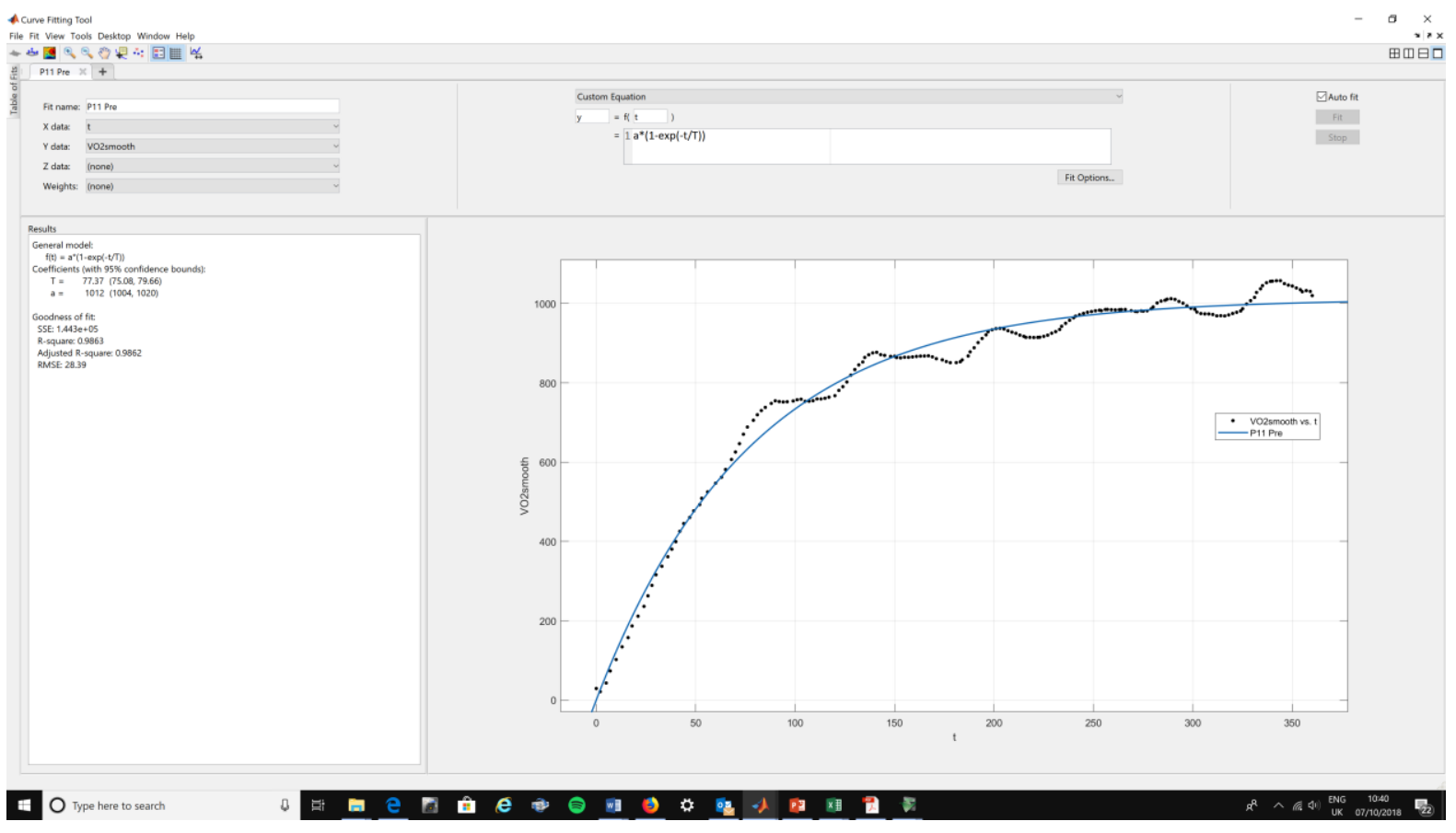


Patient 10

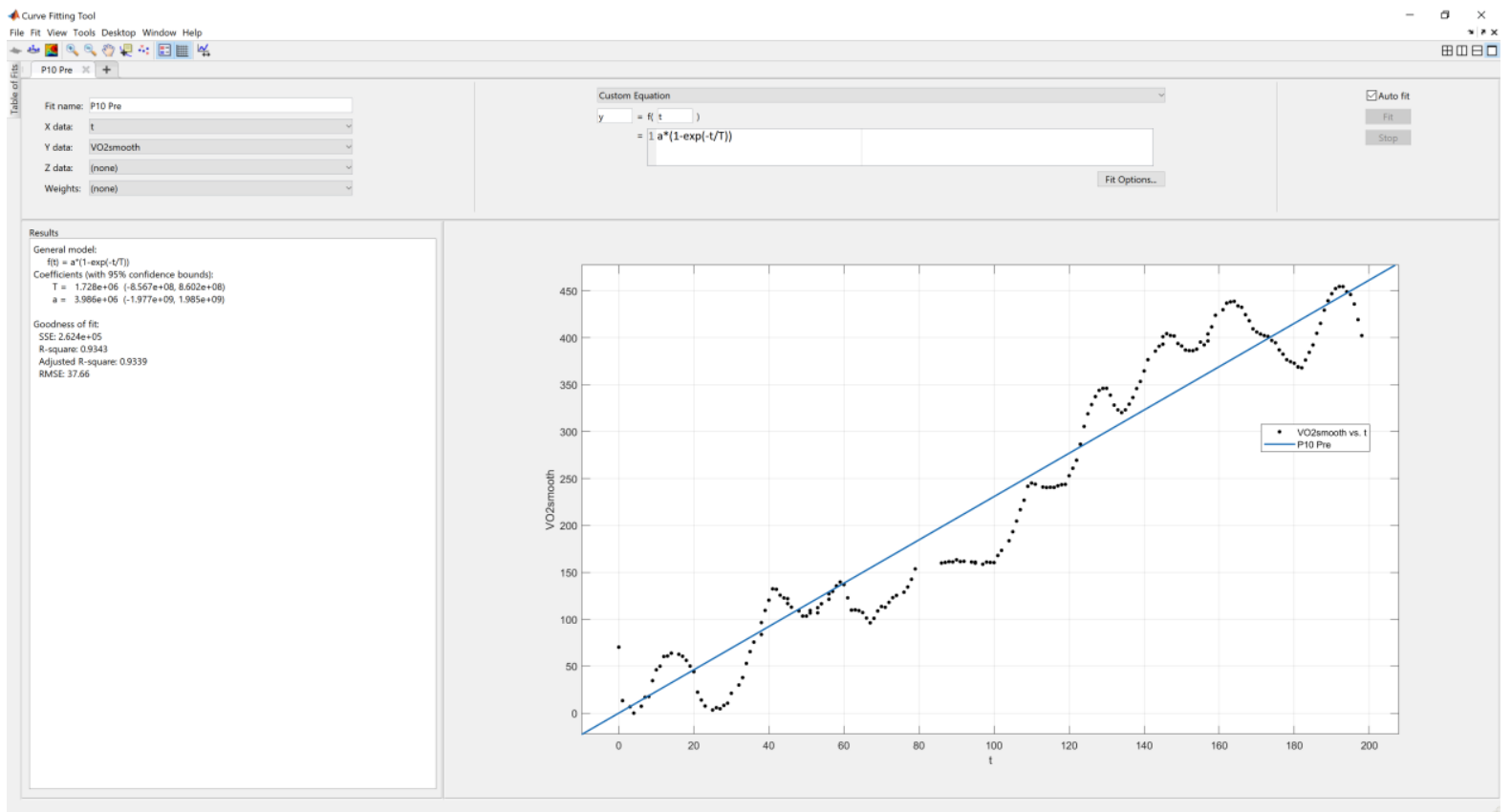

E O Type here to search

Patient 9

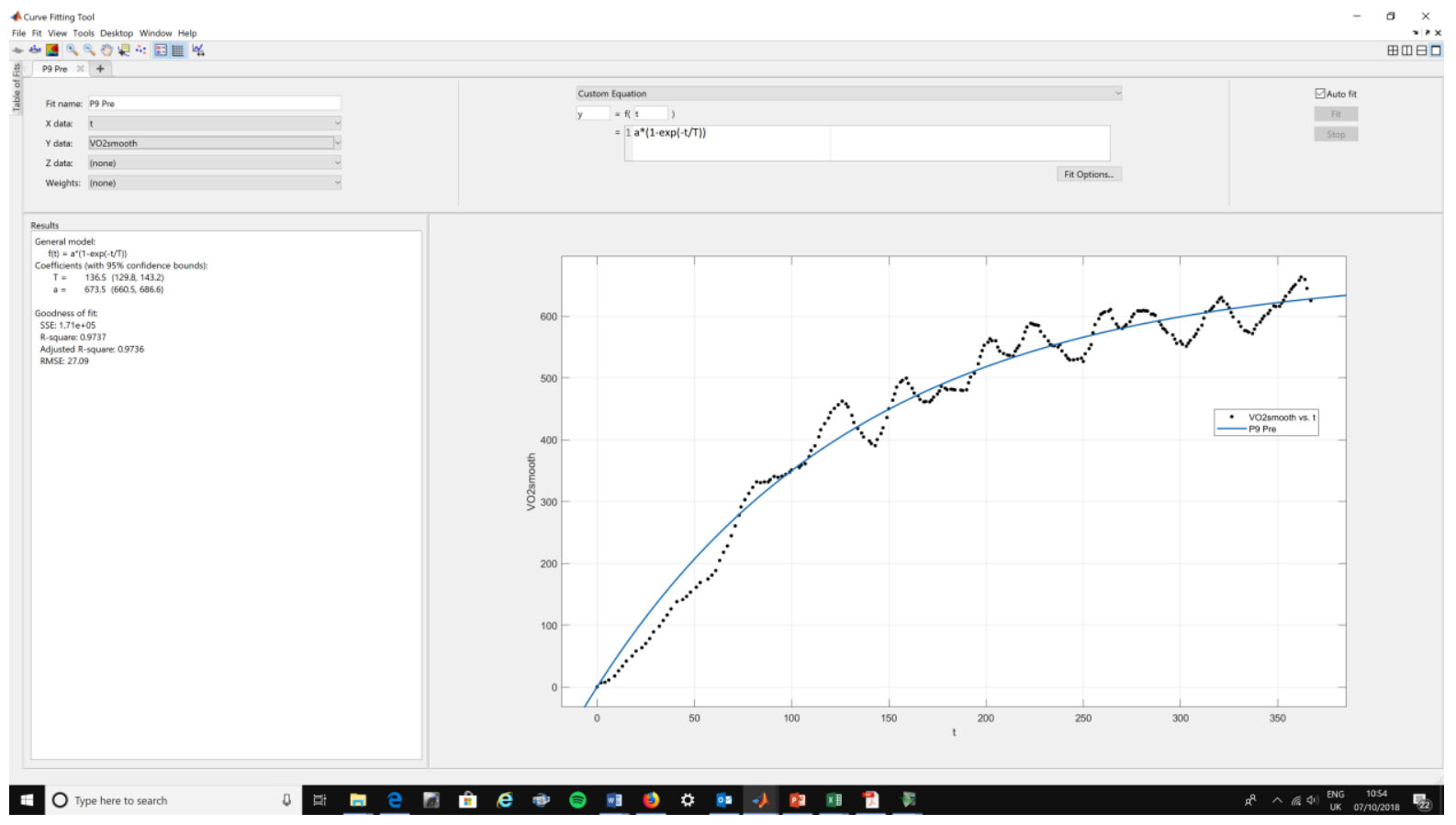




\section{Patient 8}

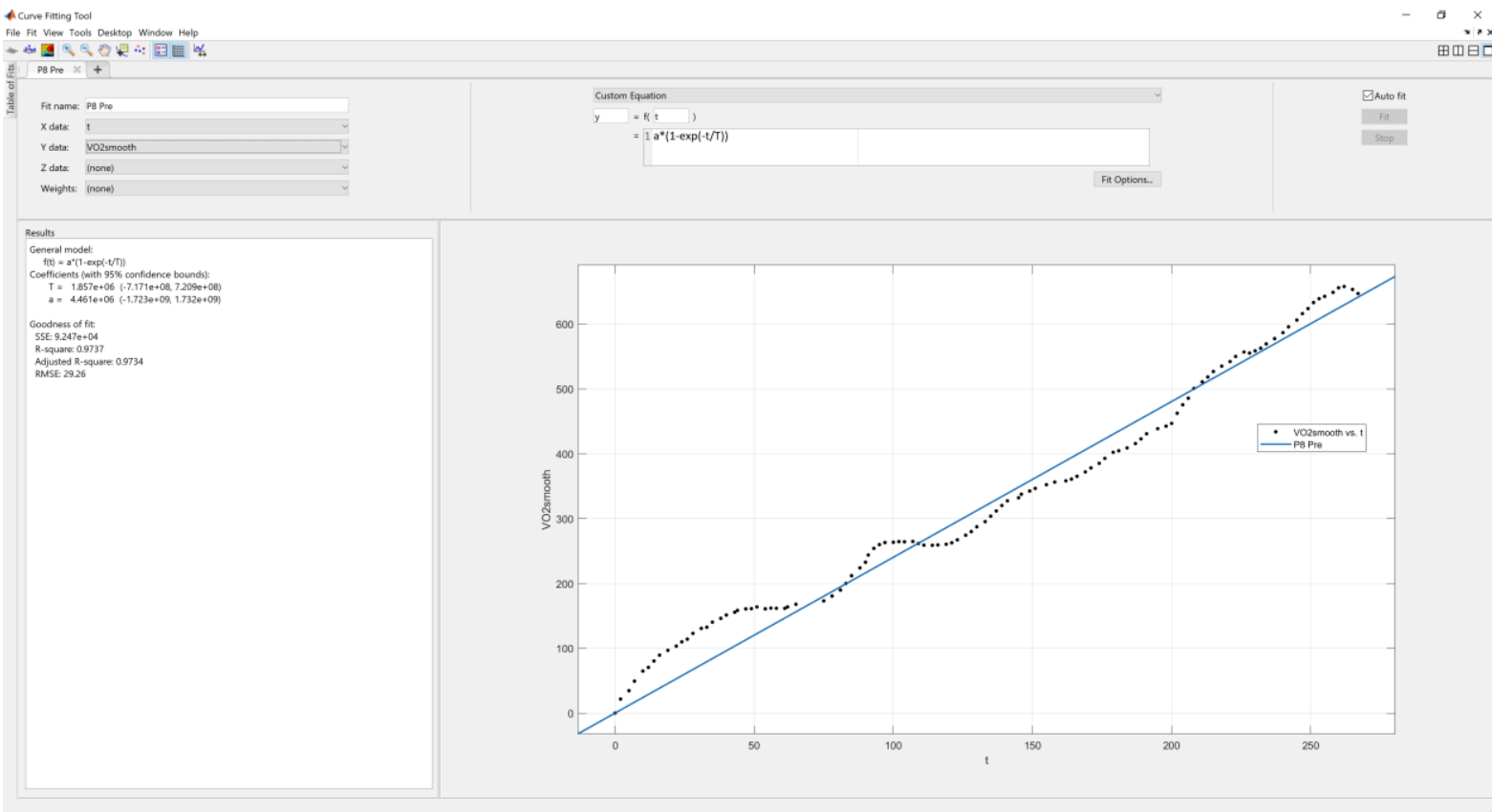

E $O$ Type here to search

Patient 7

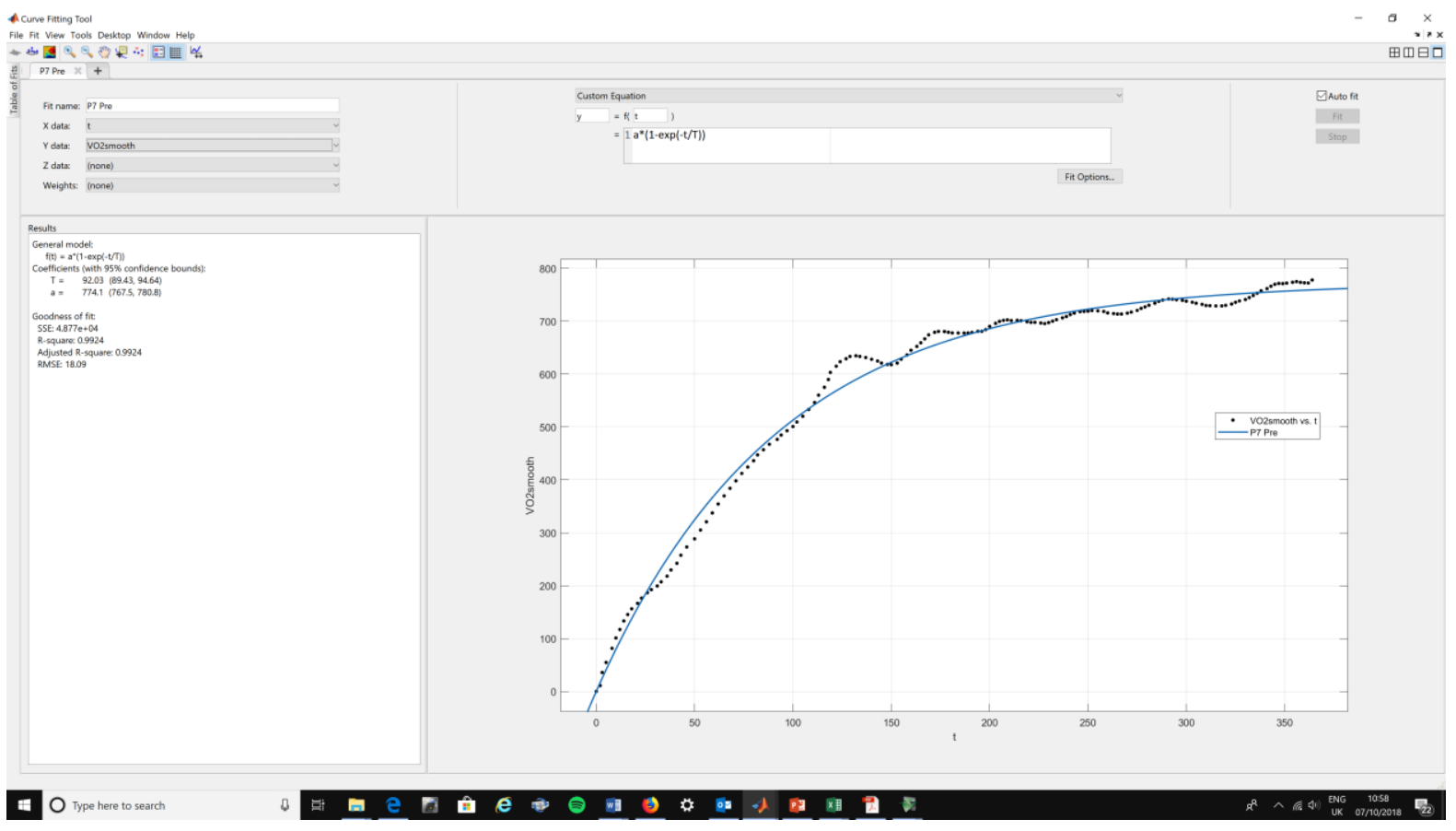




\section{Patient 6}

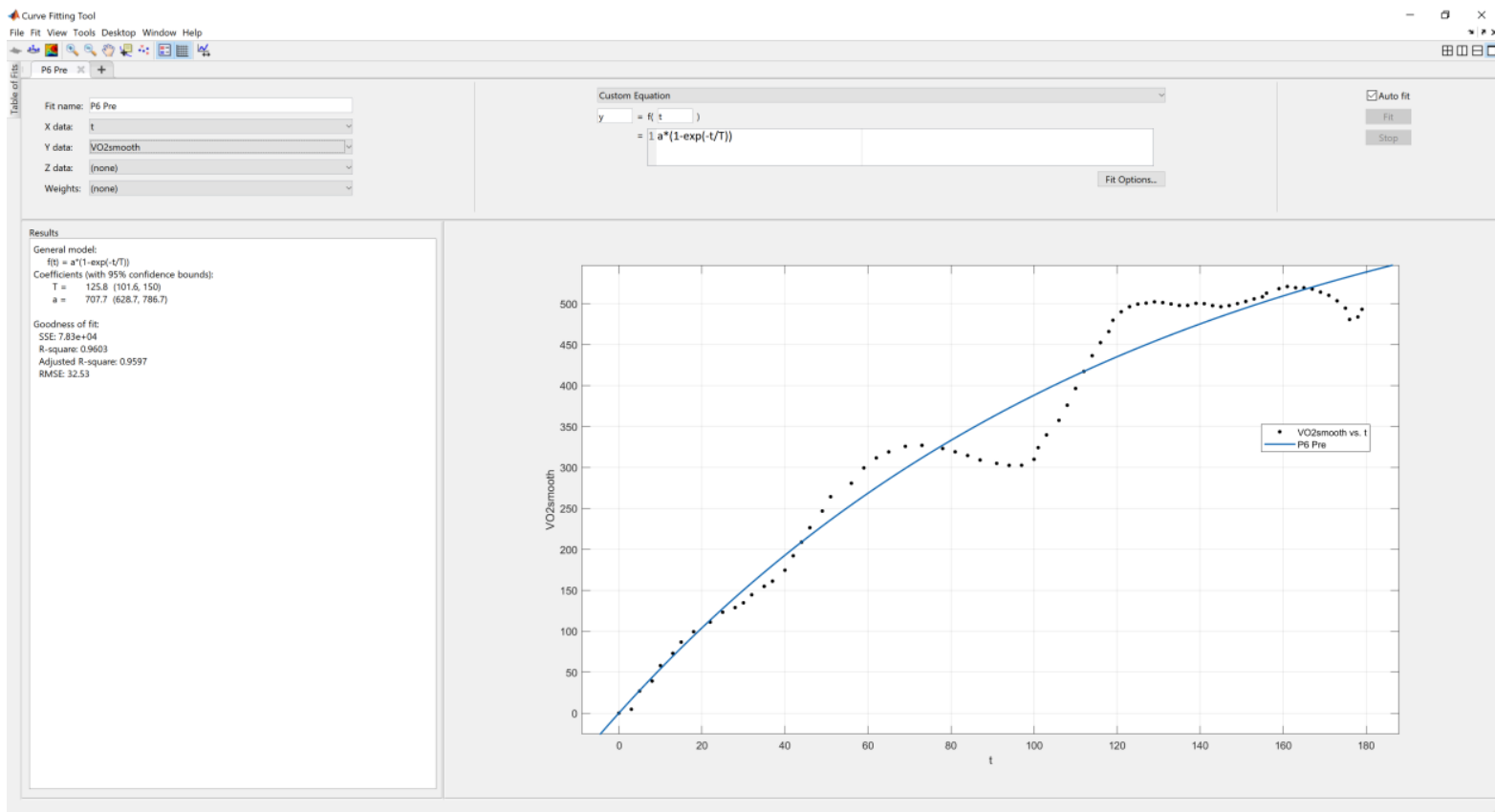

E O Type here to search

Patient 4

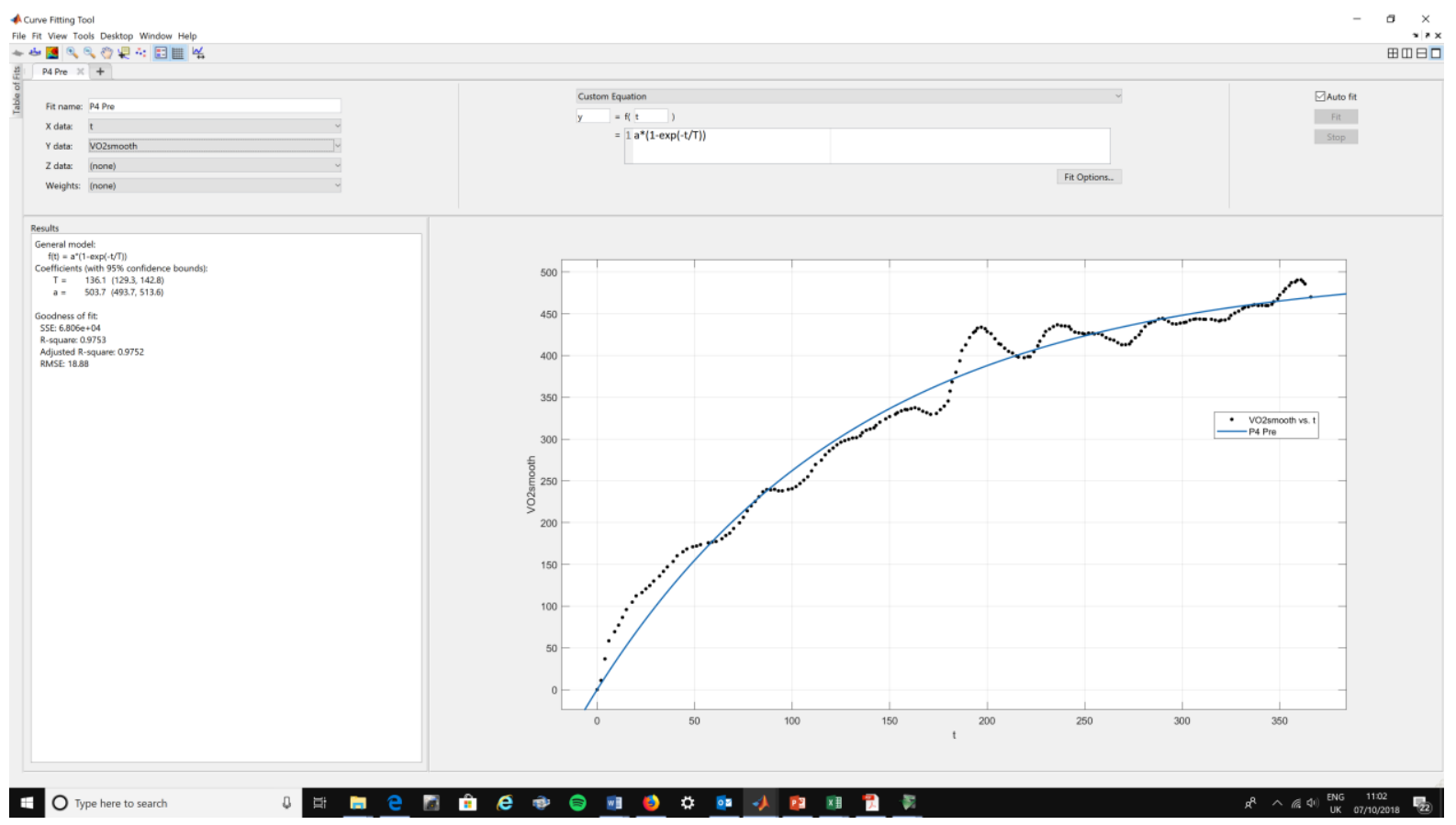




\section{Patient 3}

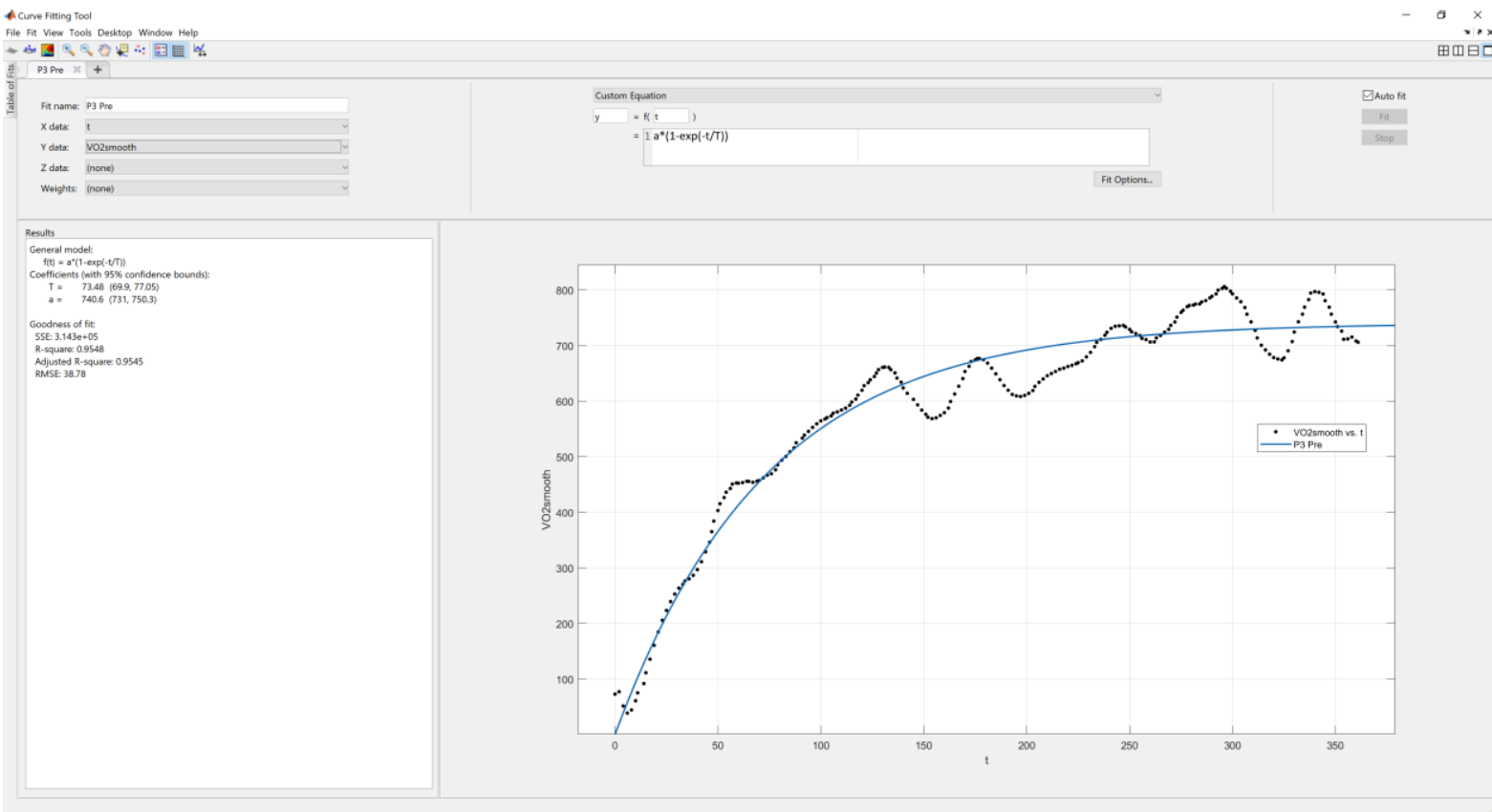

E $O$ Type here to search

Patient 1

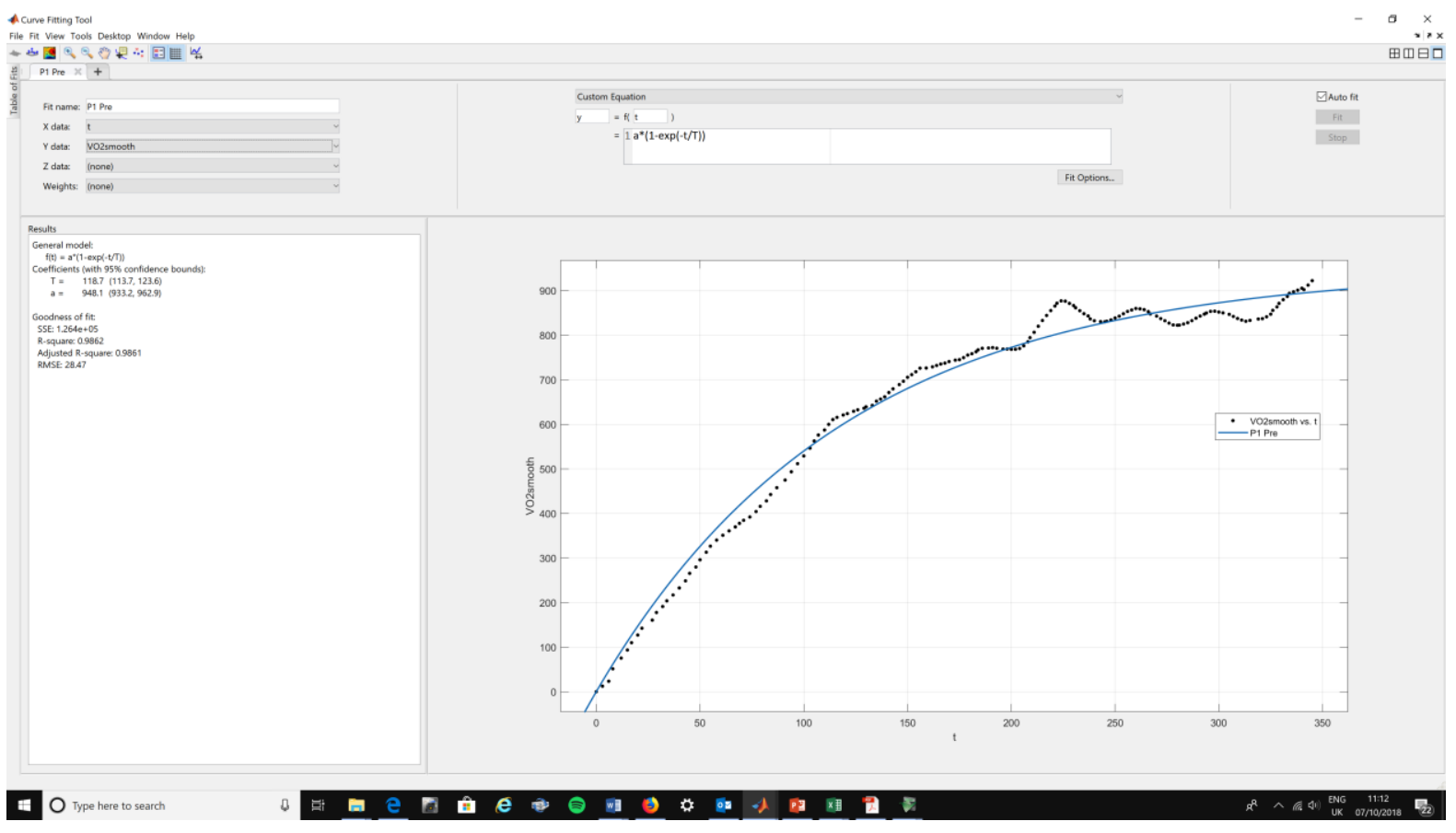


(b) Post-PR

\section{Patient 14}

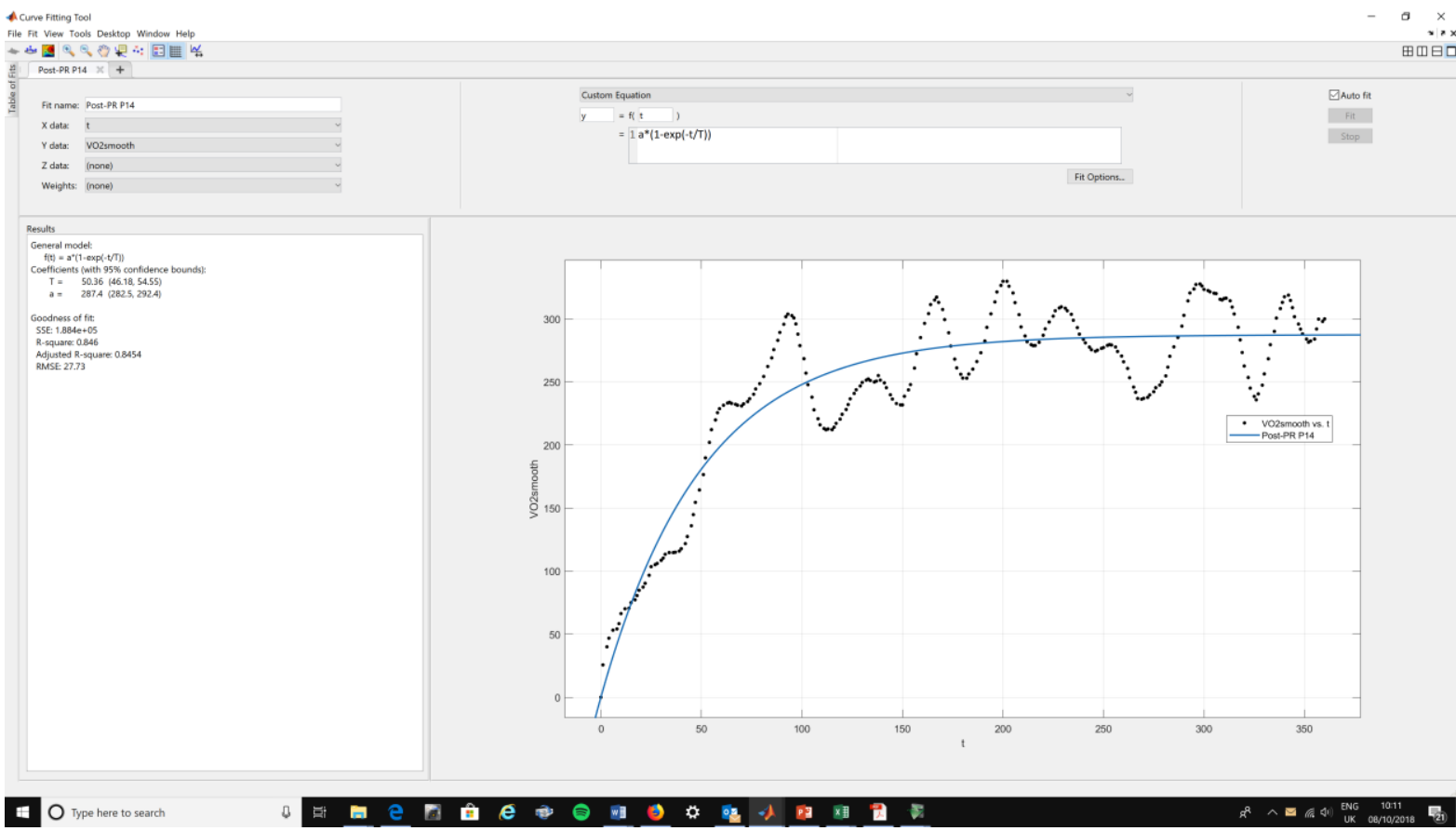

Patient 13

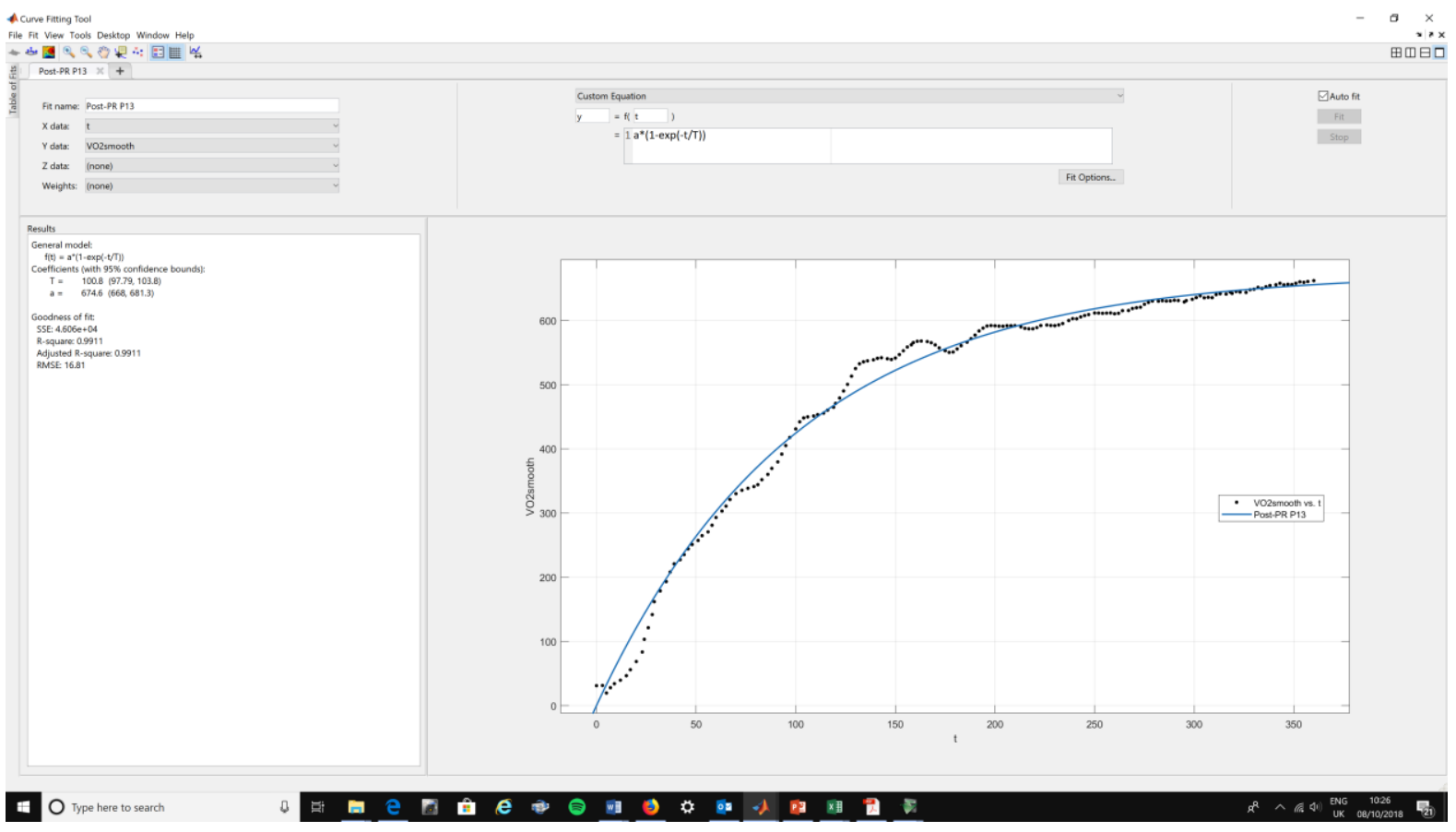


Patient 12

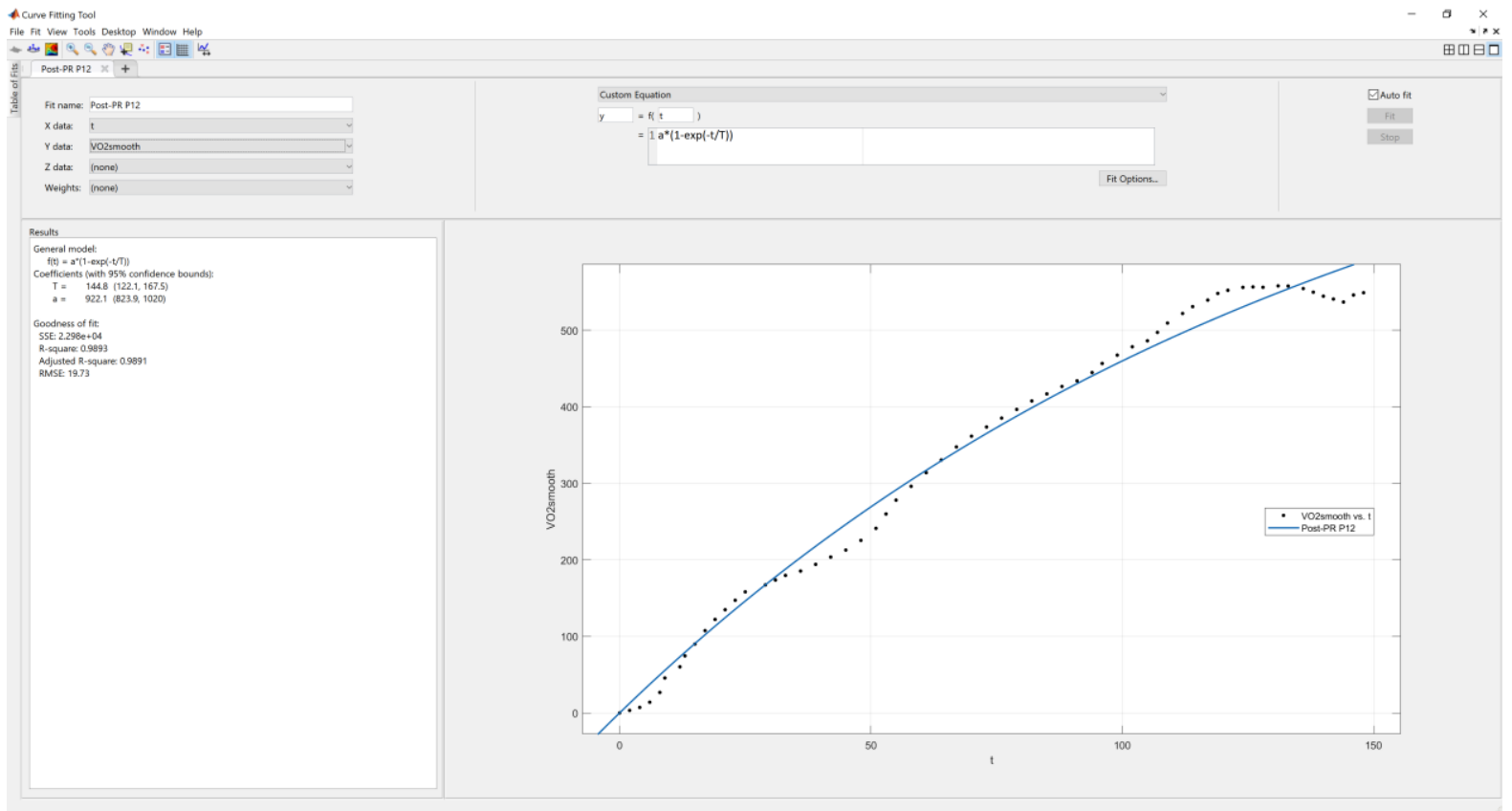

E $O$ Type here to search

Patient 11

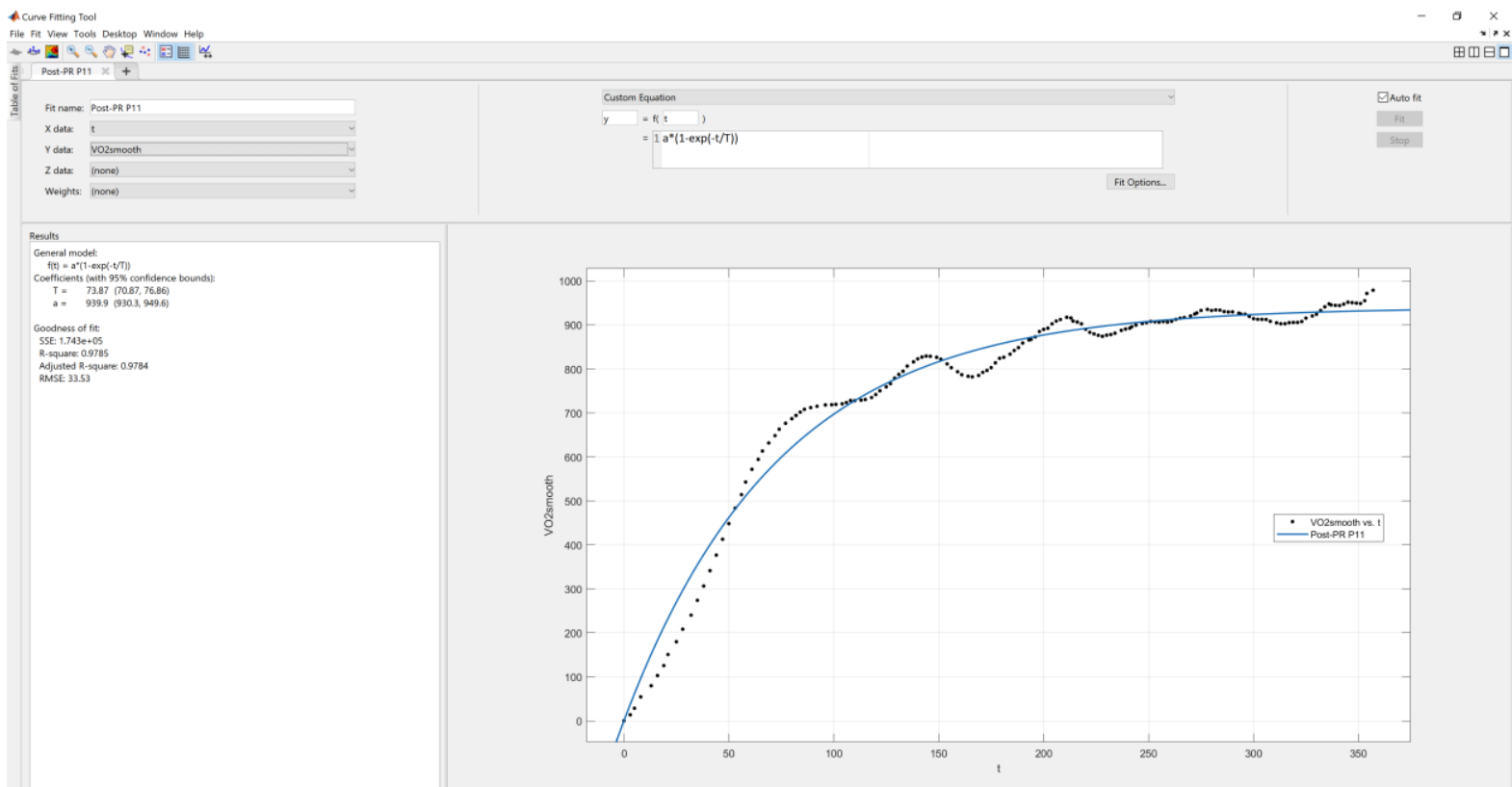

-10 
Patient 10

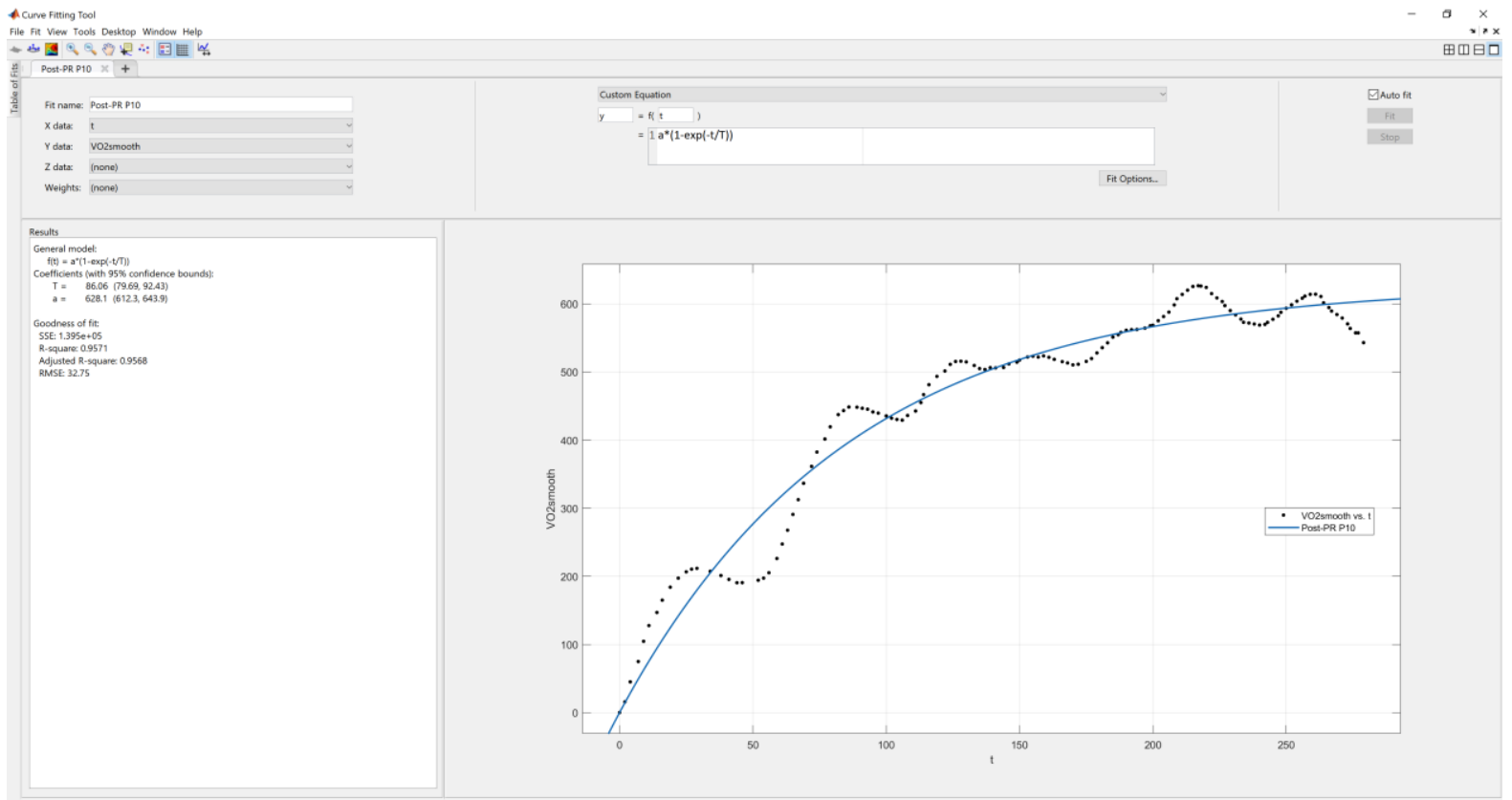

* $O$ Type here to search

Patient 9

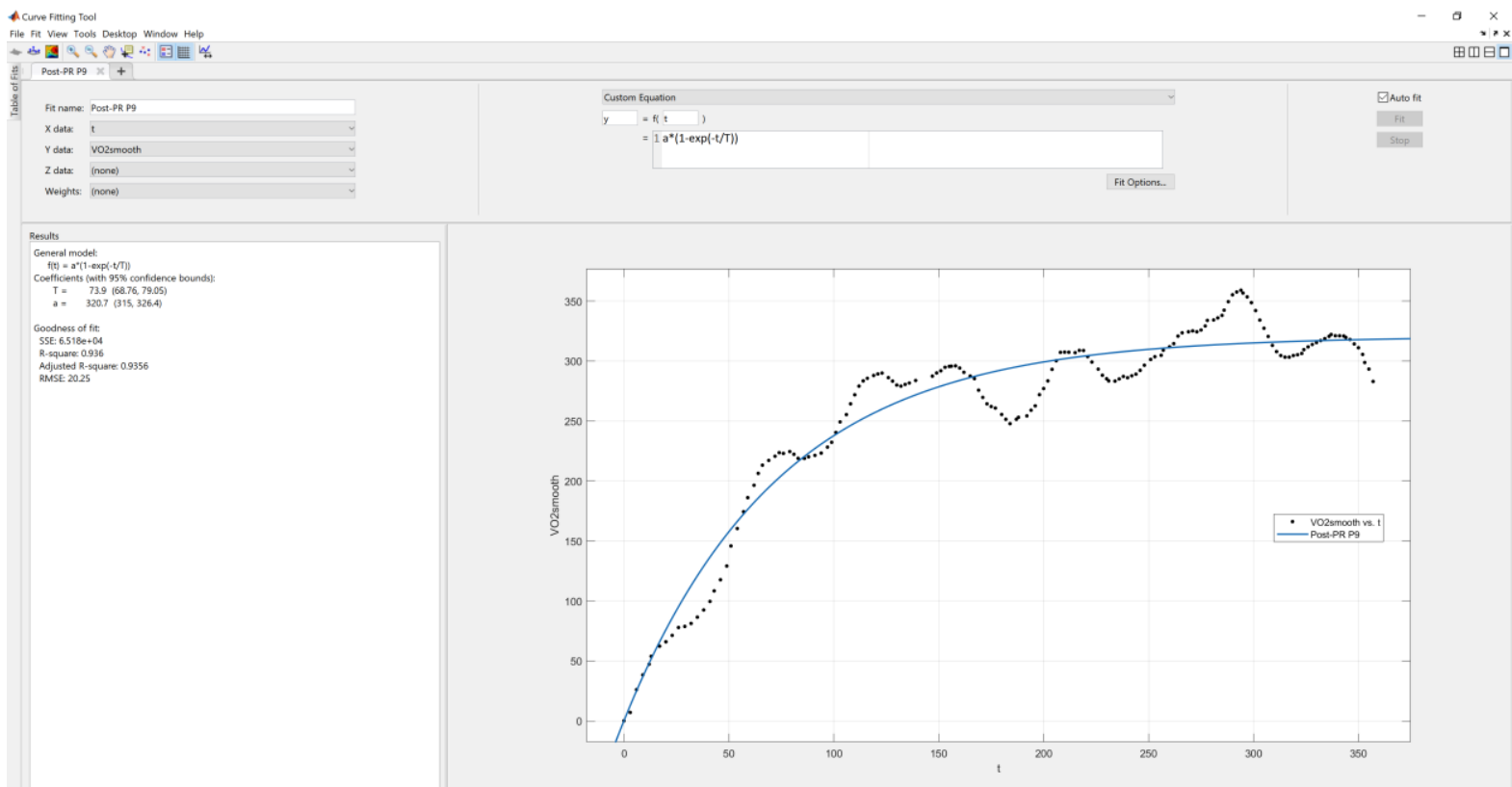




\section{Patient 8}

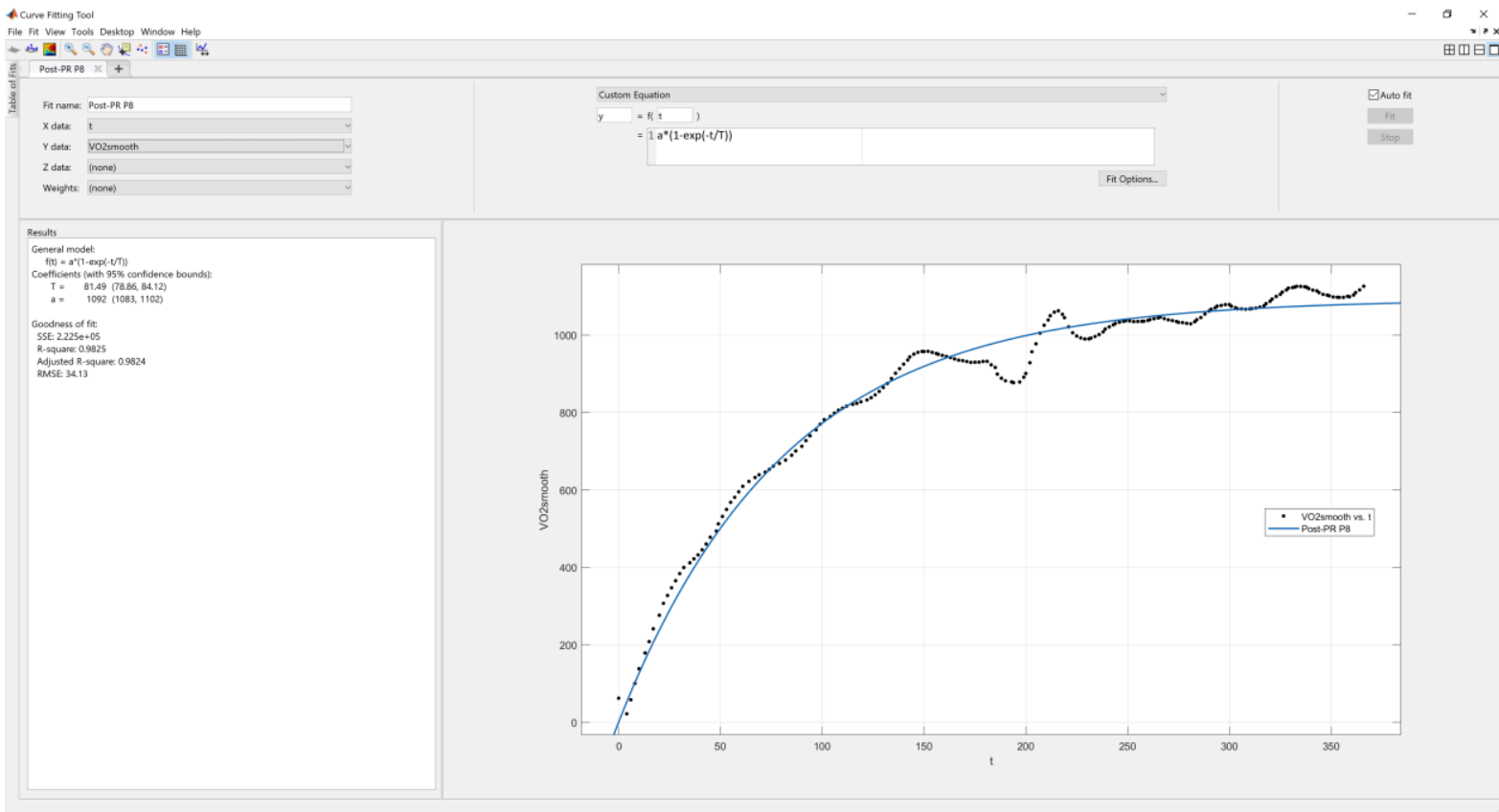

E $O$ Type here to search

Patient 7

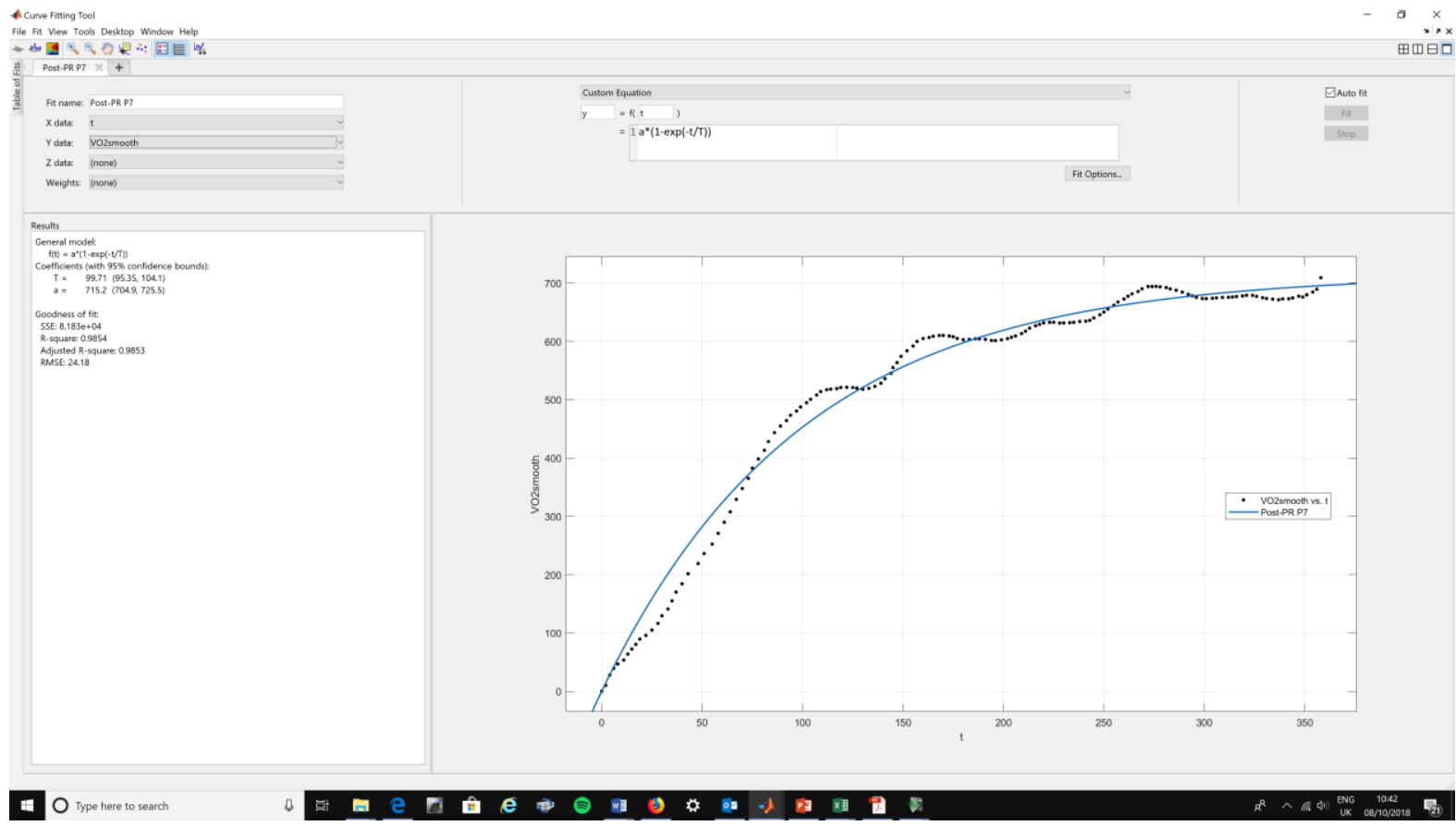




\section{Patient 6}

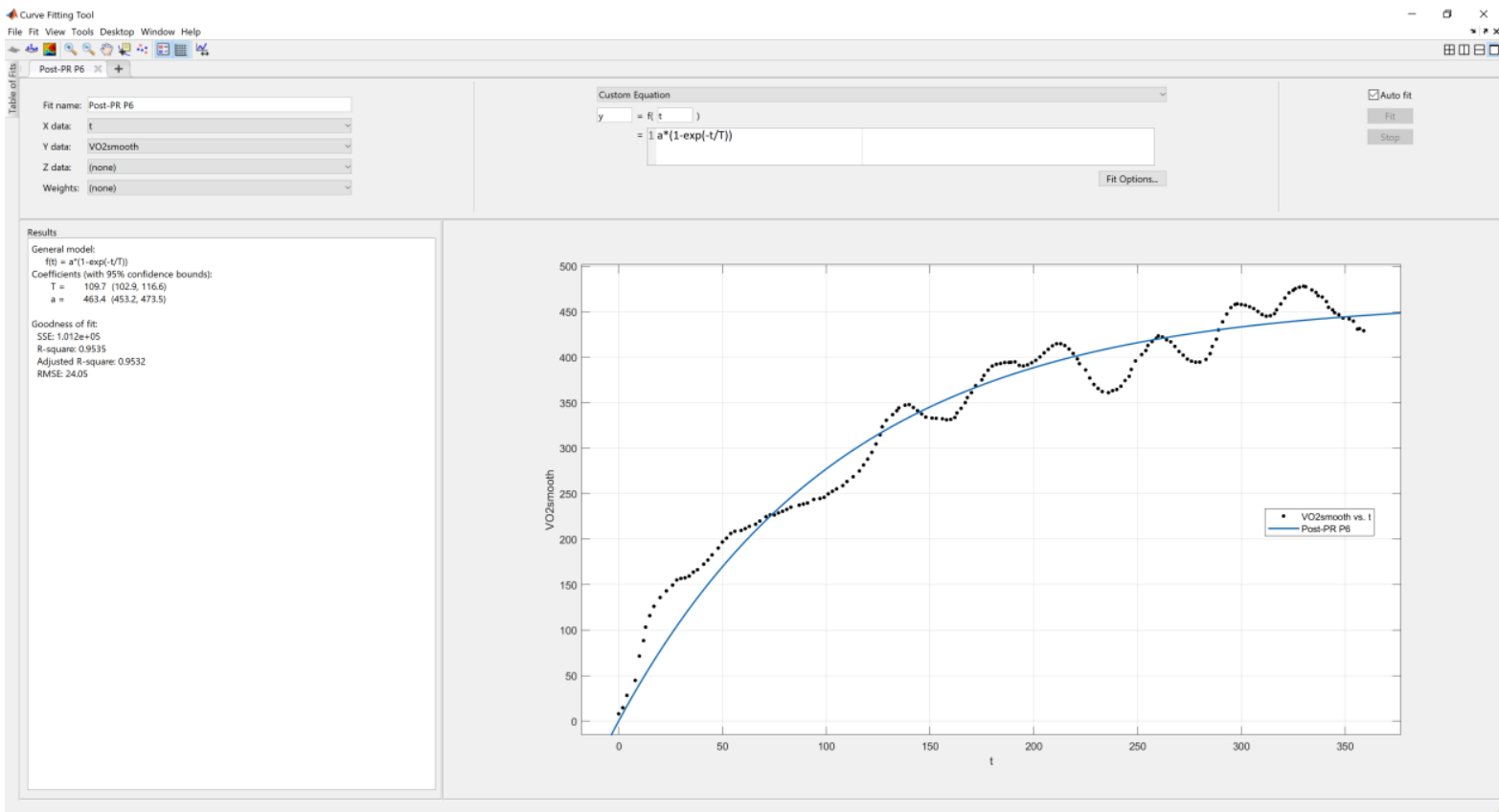

E O Type here to search

Patient 4

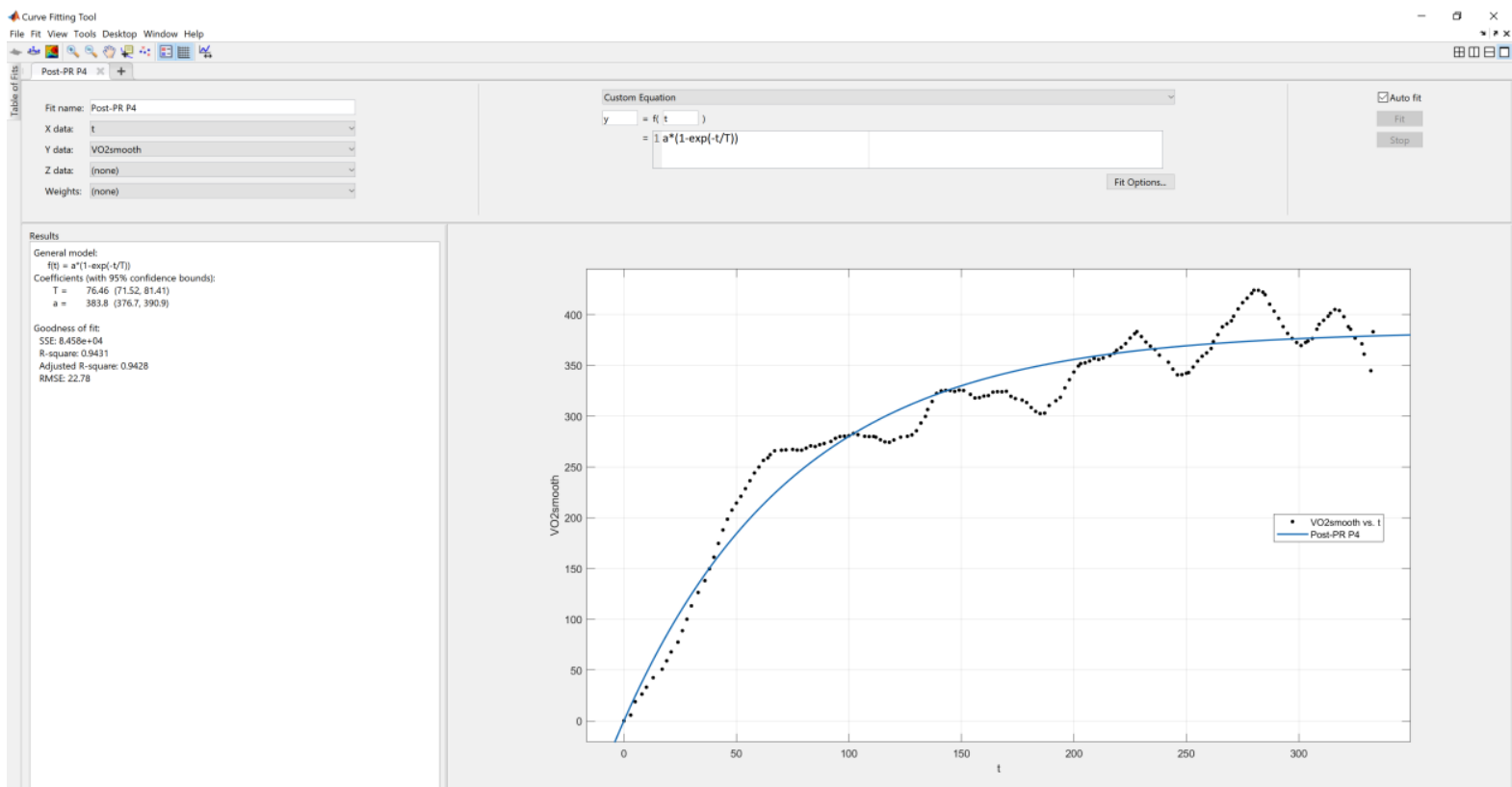




\section{Patient 3}

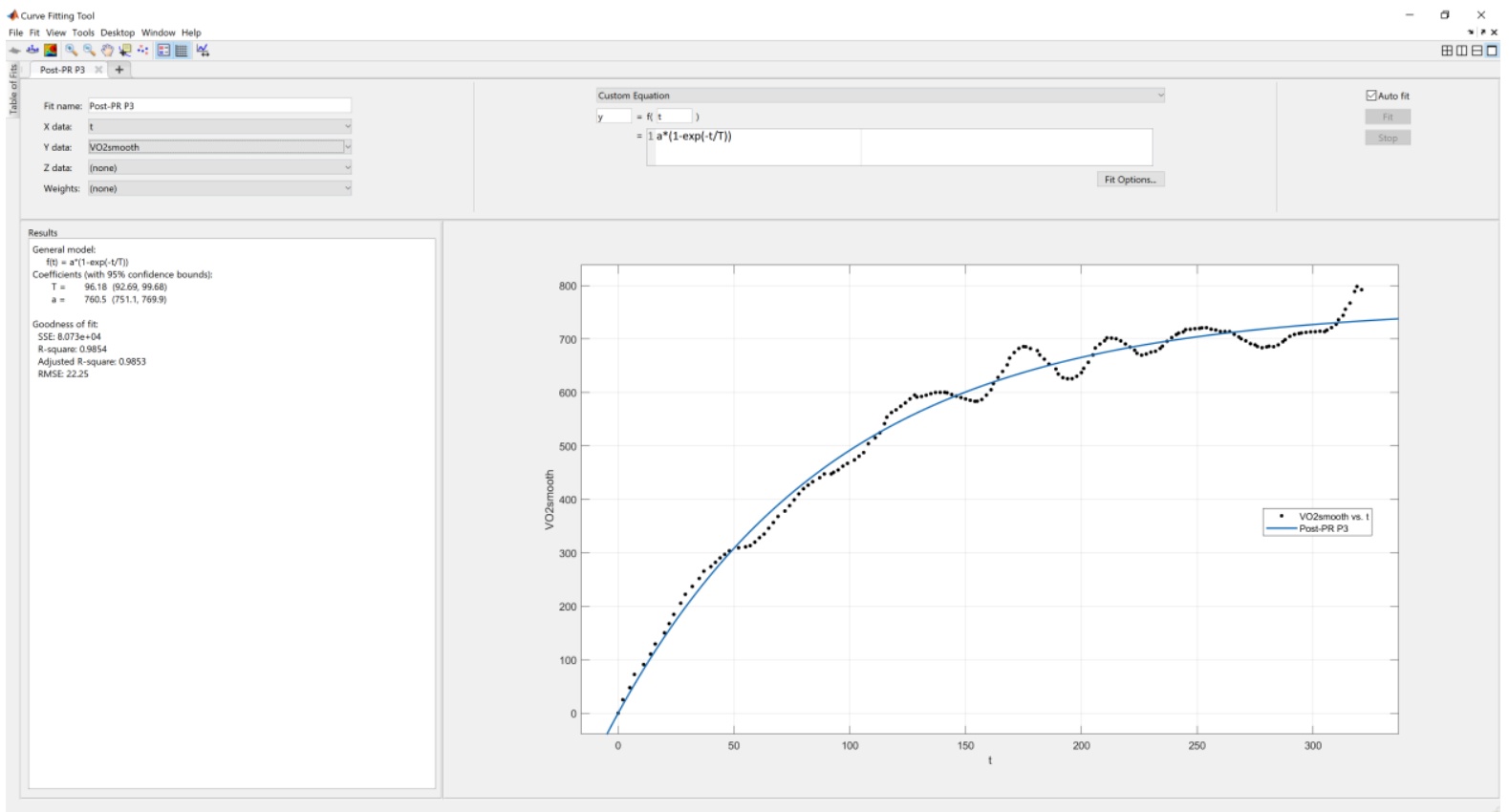

E $O$ Type here to search

Patient 1

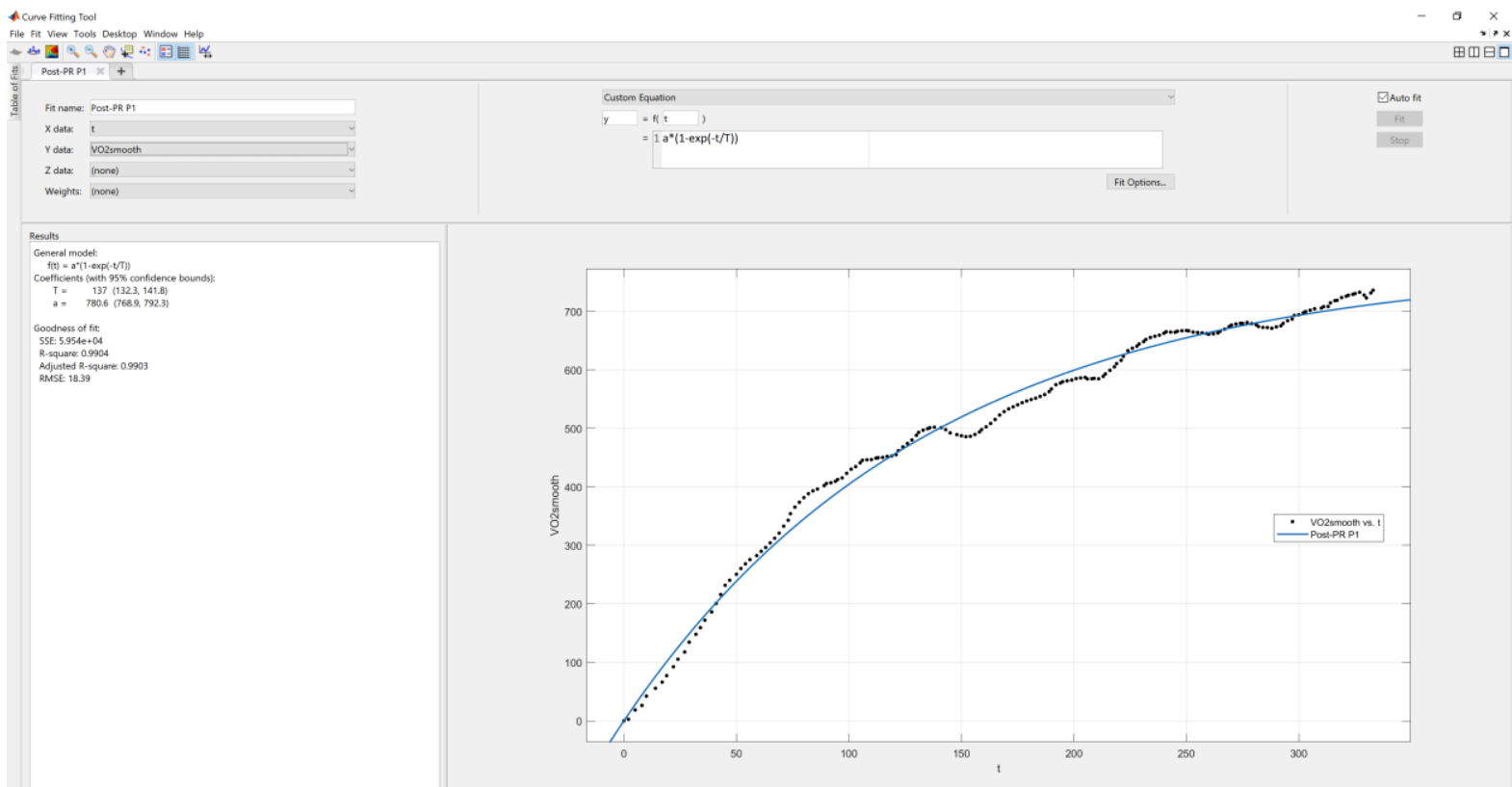


Figure D.2: Modelling of individual participants' HR data for (a) pre-PR and (b) post-PR conditions

(a) Pre -PR

\section{Patient 11}

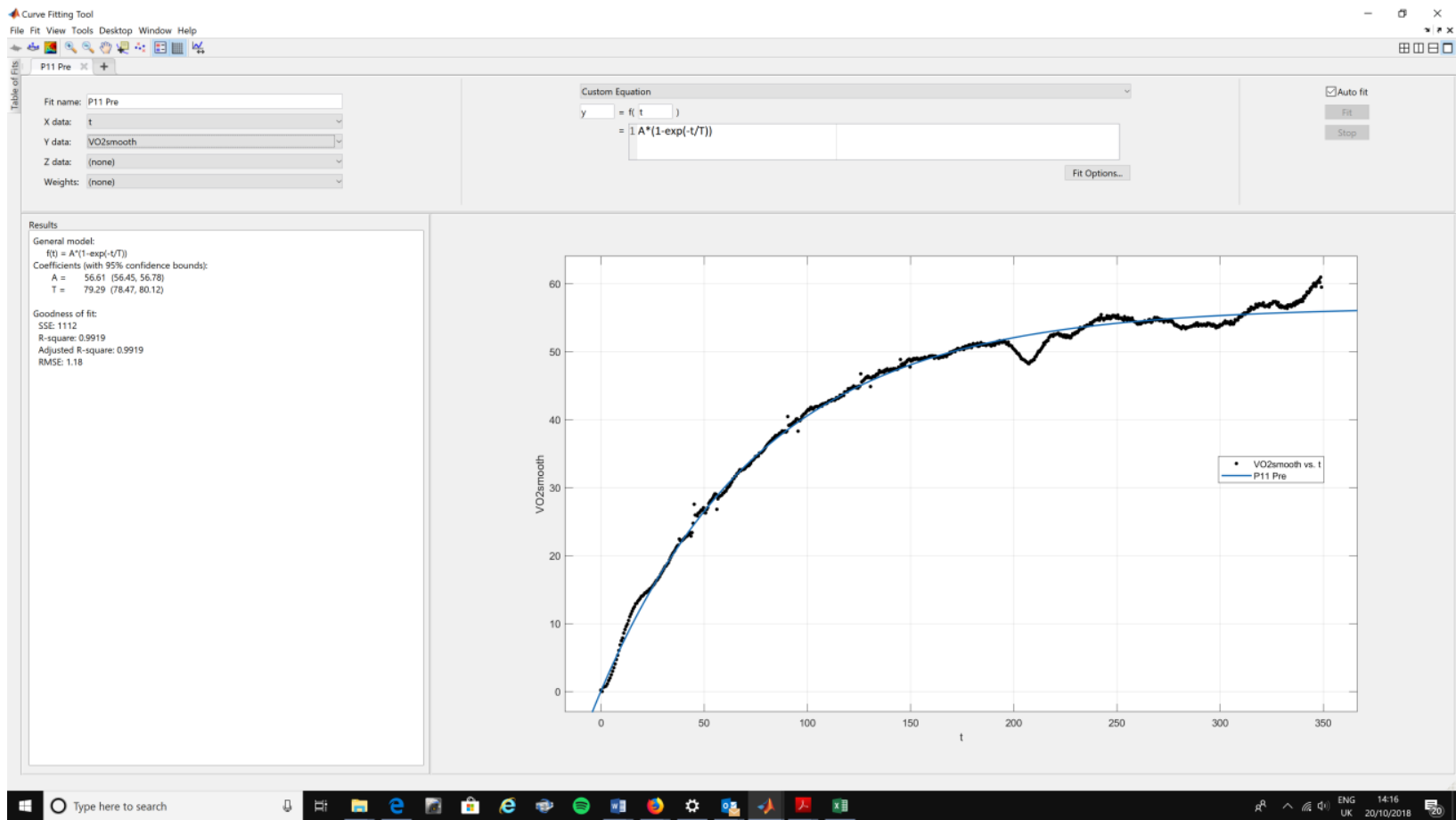

\section{Patient 9}

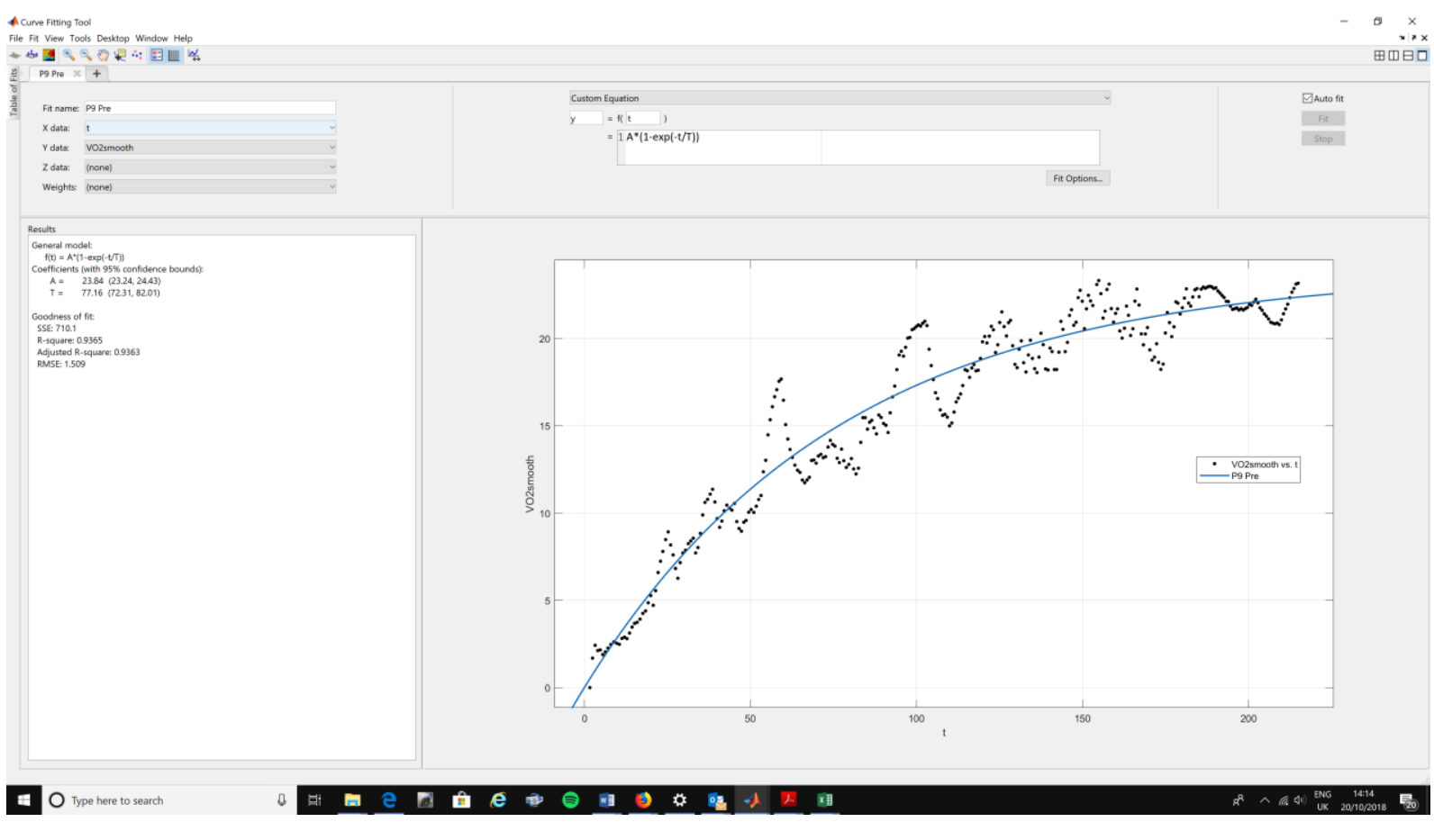




\section{Patient 8}

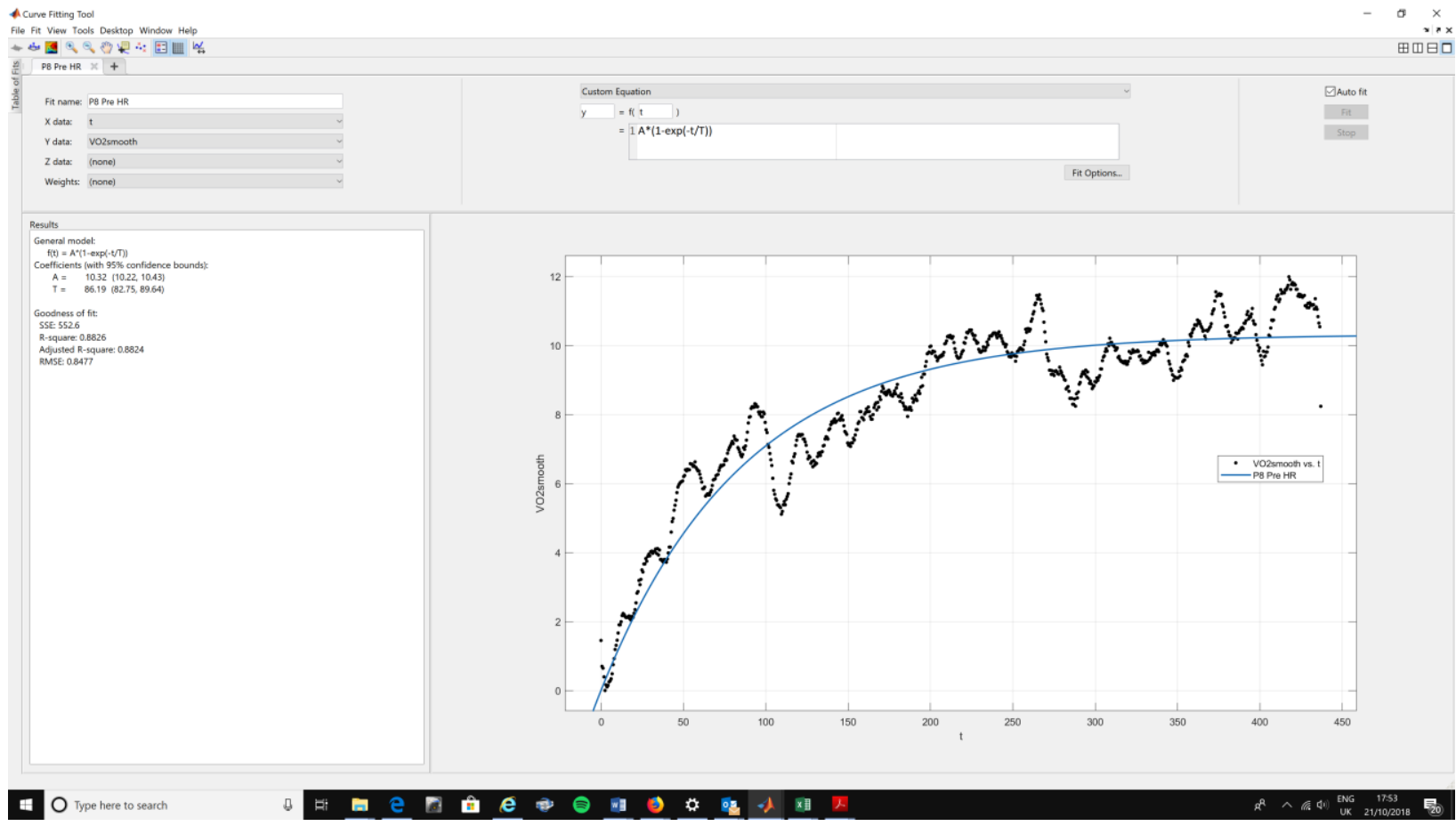

\section{Patient 6}

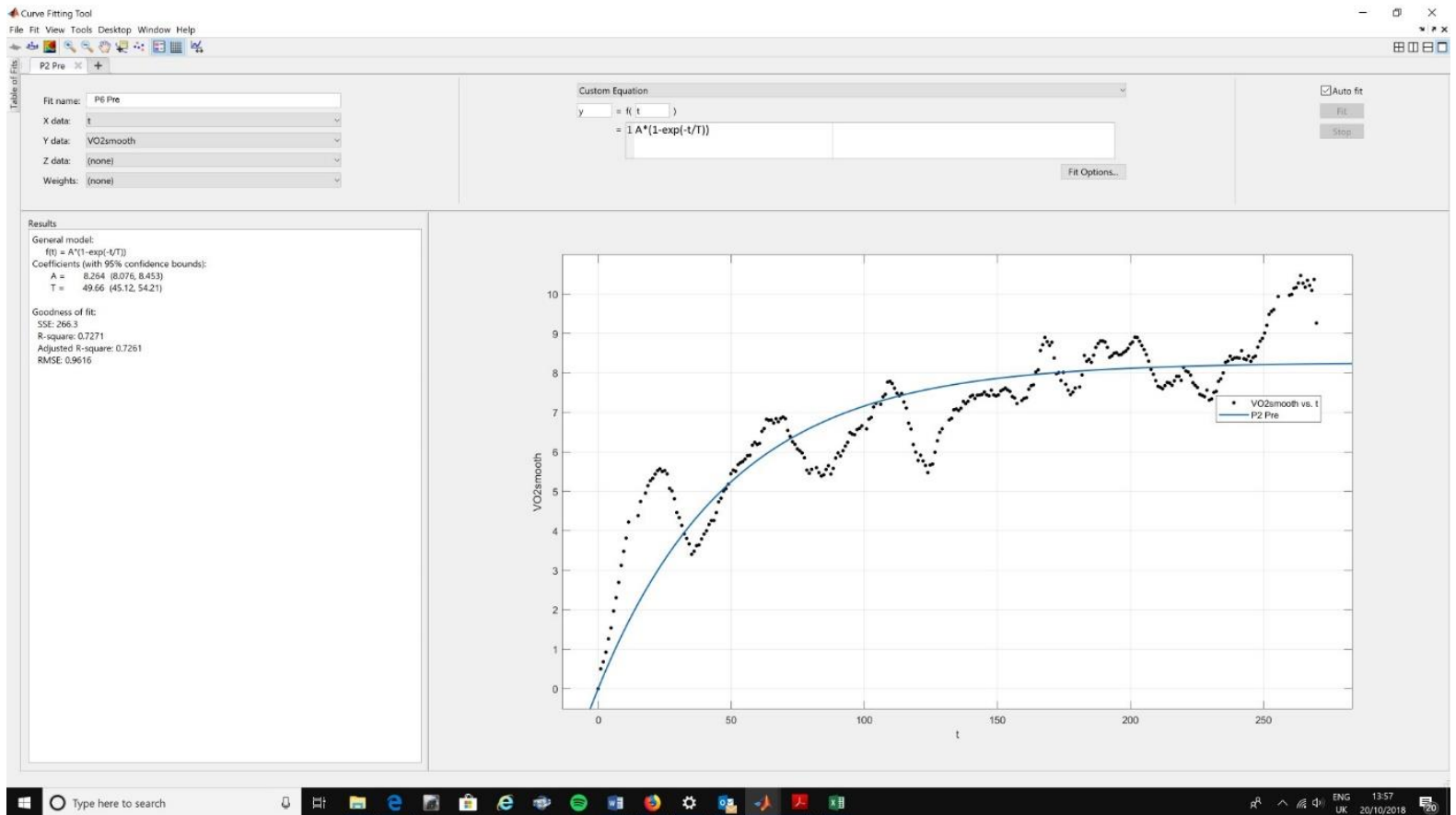

○ 


\section{Patient 4}

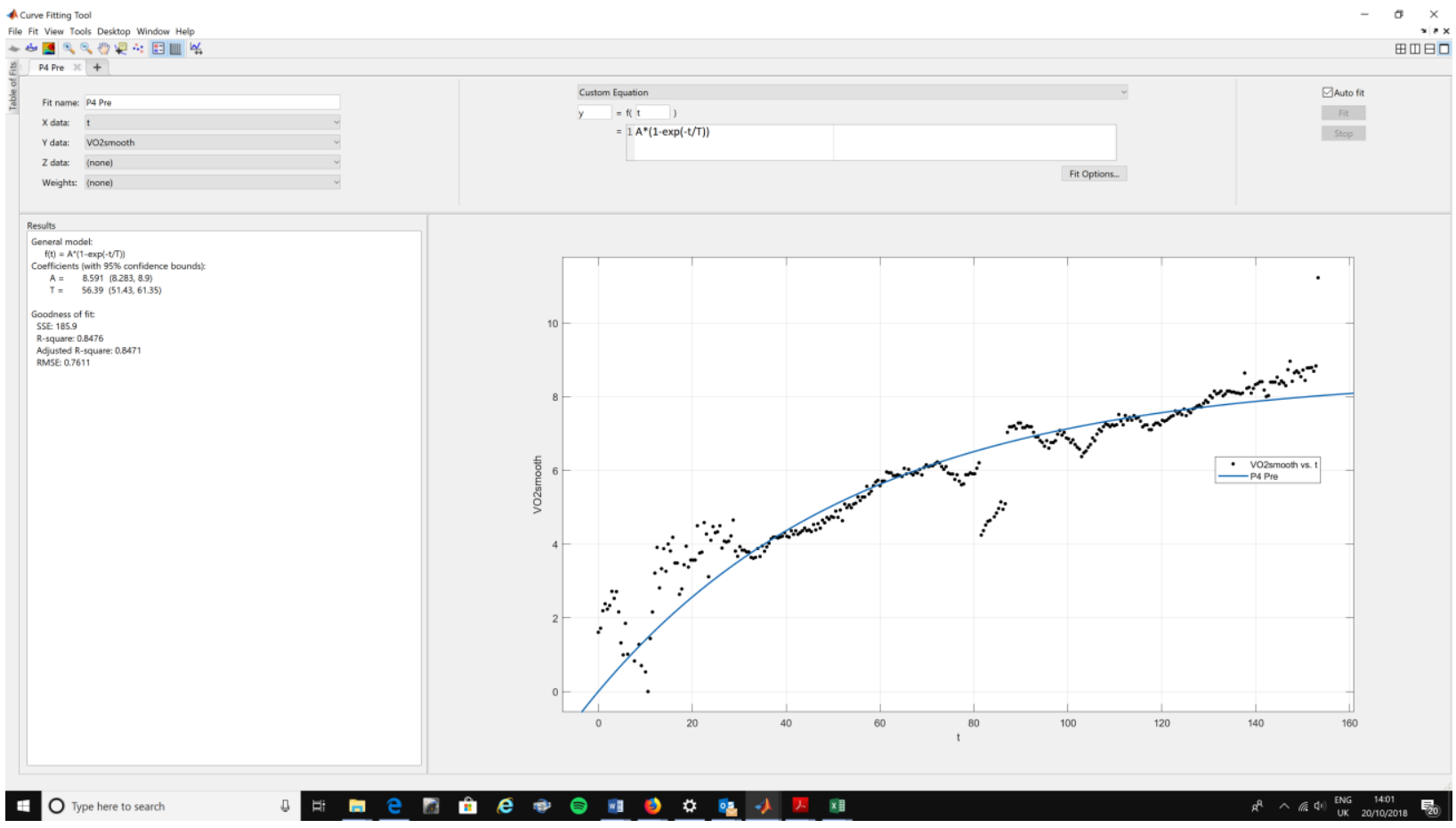

(b) Post -PR

\section{Patient 11}

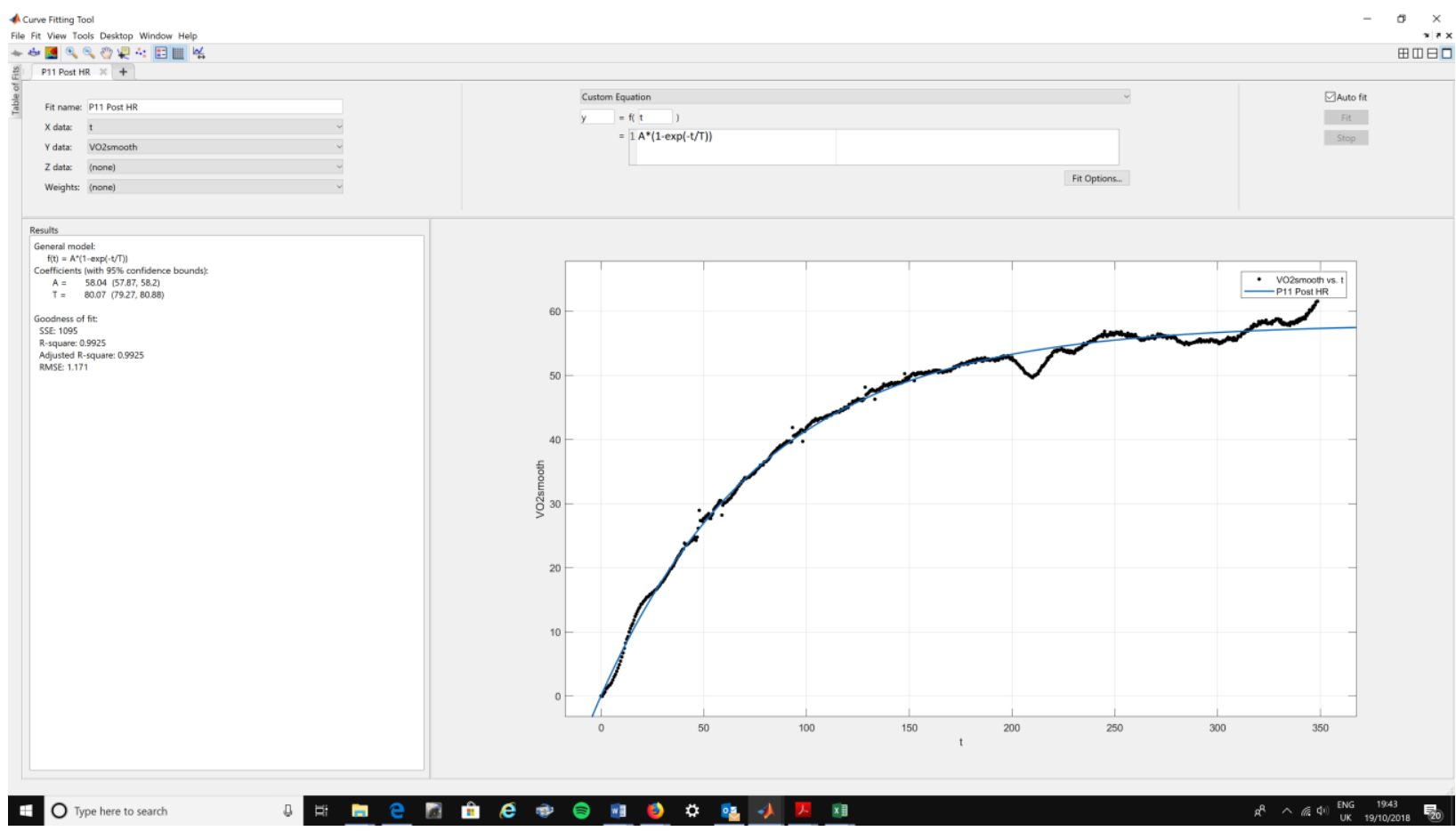




\section{Patient 9}

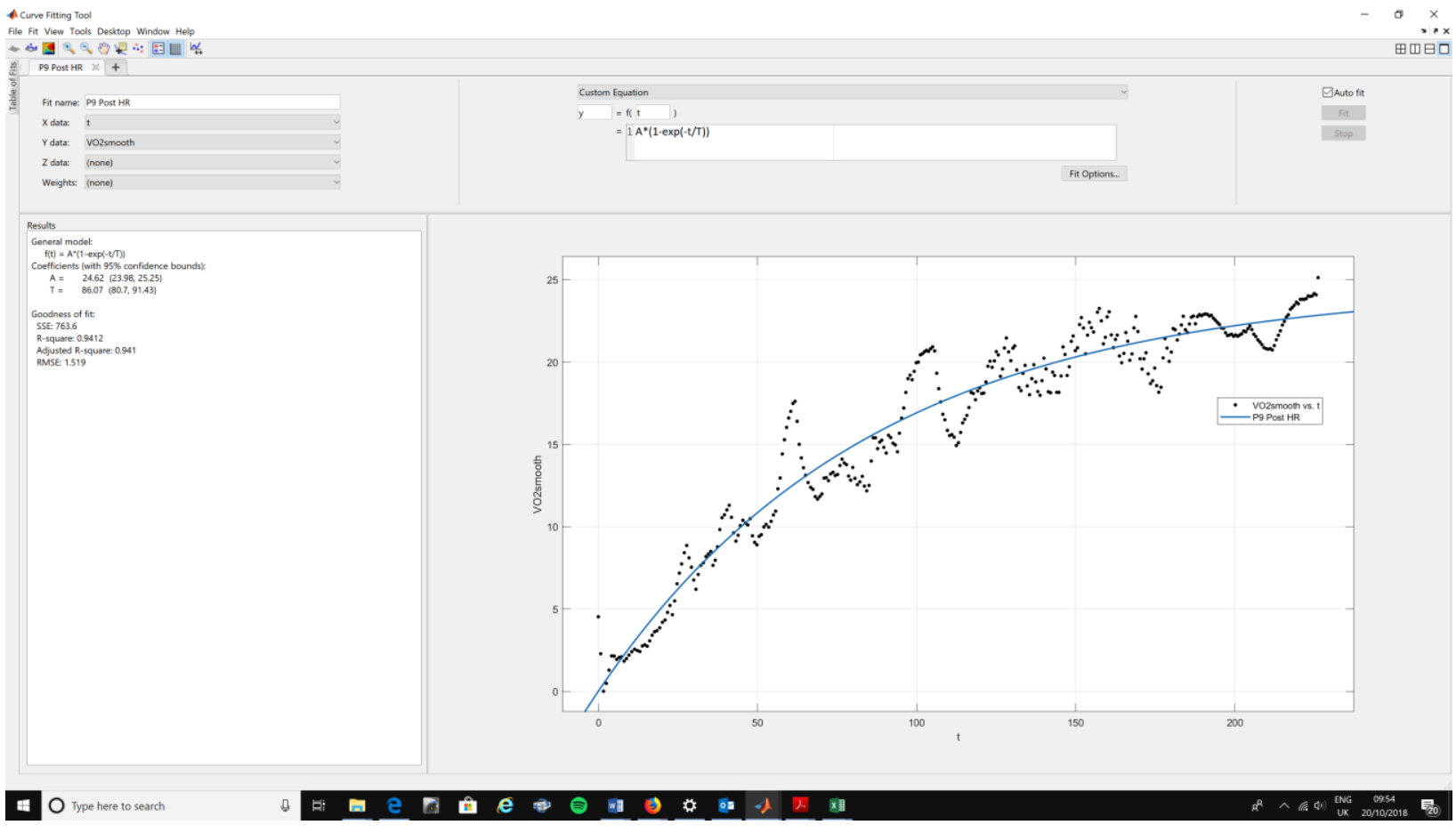

\section{Patient 8}

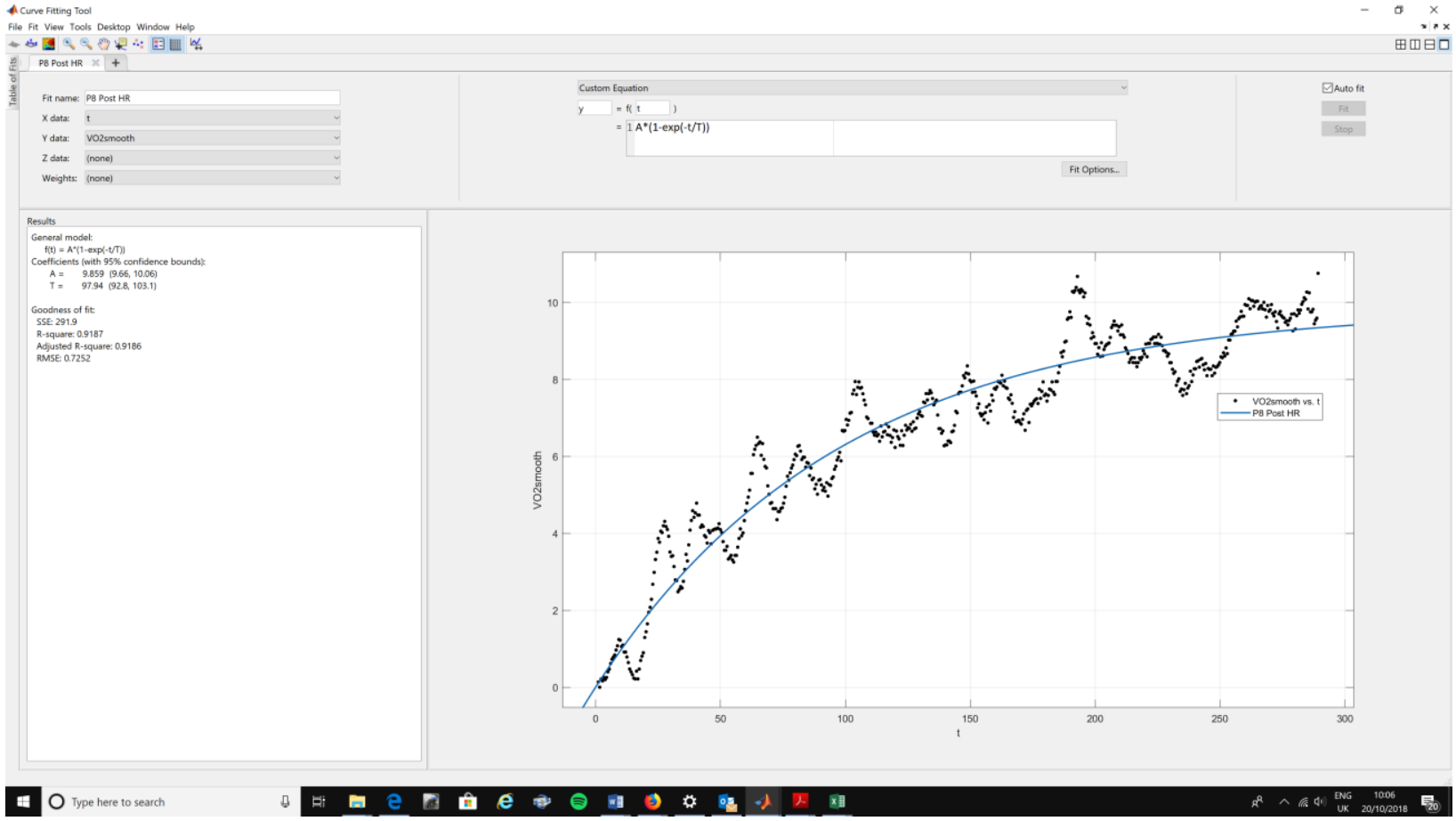




\section{Patient 6}

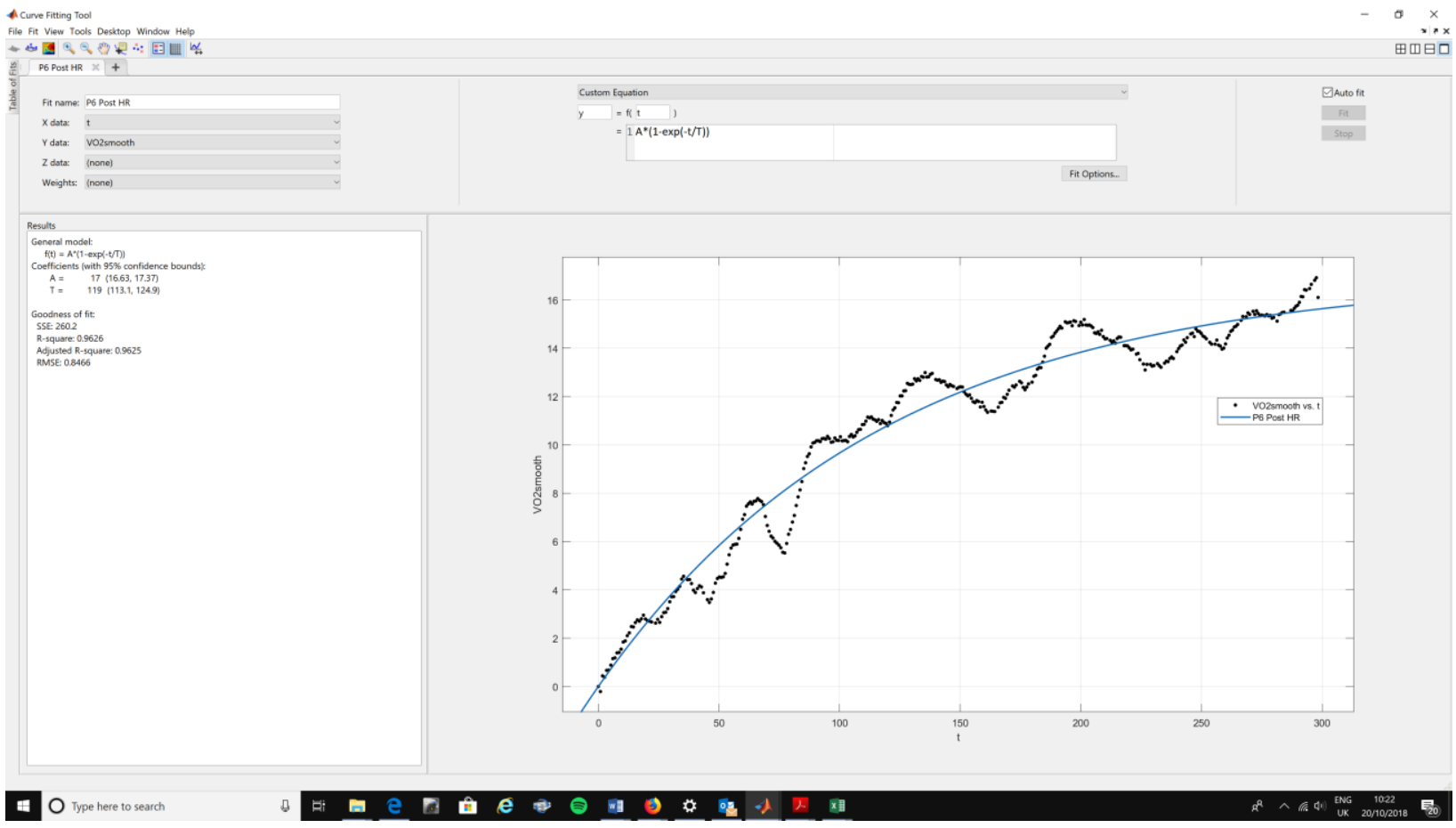

Patient 4

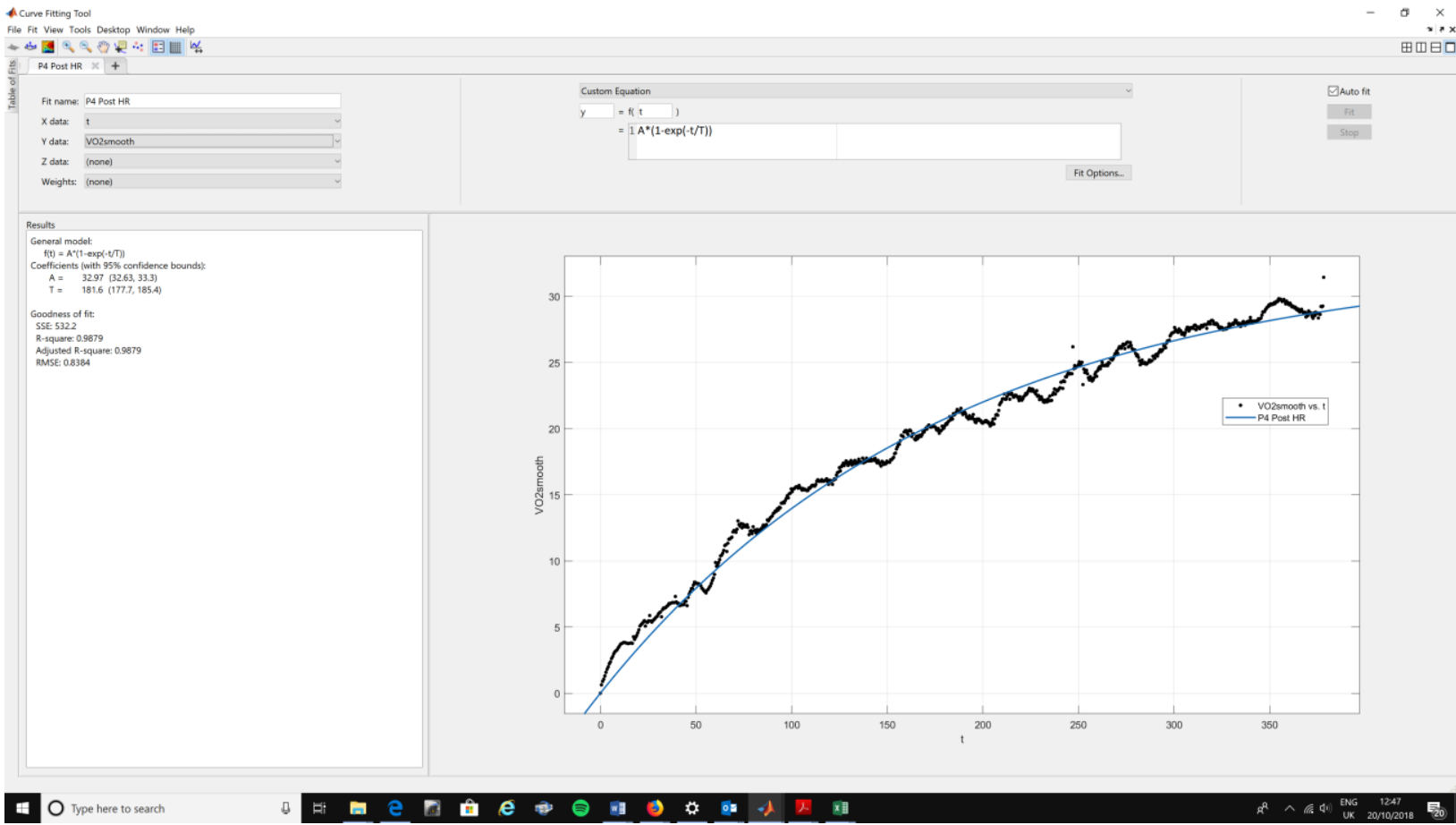


Appendix E: Example of an efficacy 'staging' system for pulmonary rehabilitation in IPF

\begin{tabular}{|c|c|c|c|c|c|}
\hline \multicolumn{6}{|c|}{$\begin{array}{l}\text { Figure E.1: Proposed staging system for pulmonary rehabilitation for IPF. Green, yellow } \\
\text { and orange represent low, moderate and high disease severity } \\
\text { Male }\end{array}$} \\
\hline \multicolumn{3}{|c|}{ Reference values (based on FVC \%) } & \multirow{2}{*}{$\begin{array}{c}\text { Pre } \\
\text { rehabilitation }\end{array}$} & \multirow{2}{*}{$\begin{array}{c}\text { Post } \\
\text { rehabilitation }\end{array}$} & \multirow[b]{2}{*}{$\%$ change } \\
\hline$>80$ & $80-55$ & $<55$ & & & \\
\hline \multicolumn{6}{|c|}{ Ve (BPTS) } \\
\hline 67 & 57 & 52 & & & \\
\hline \multicolumn{6}{|c|}{$\mathrm{VO}_{2}(\mathrm{ml} \cdot \mathrm{kg}-1 \cdot \mathrm{min}-1)$} \\
\hline 19 & 15 & 14 & & & \\
\hline \multicolumn{6}{|c|}{ Peak HR (BPM) } \\
\hline 117 & 115 & 101 & & & \\
\hline \multicolumn{6}{|c|}{ Peak WR (Watts) } \\
\hline 107 & 101 & 94 & & & \\
\hline \multicolumn{6}{|c|}{ Time to peak (min) } \\
\hline 14 & 11 & 9 & & & \\
\hline \multicolumn{6}{|c|}{ Oxygen saturation at peak VO2 } \\
\hline 89 & 84 & 84 & & & \\
\hline \multicolumn{6}{|c|}{ six-minute walk test $(\mathrm{m})$} \\
\hline 319 & 286 & 222 & & & \\
\hline BMI (pre) & BMI (Post) & FVC\% (Pre) & FVC\% (Post) & & \\
\hline & & & & & \\
\hline & & & & & \\
\hline \multicolumn{6}{|c|}{ Female } \\
\hline \multicolumn{3}{|c|}{ Reference values (based on FCV \%) } & & & \\
\hline$>80$ & $80-55$ & $<55$ & rehabilitation & rehabilitation & $\%$ change \\
\hline \multicolumn{6}{|c|}{ six-minute walk test $(\mathrm{m})$} \\
\hline 384 & 358 & 310 & & & \\
\hline \multicolumn{6}{|c|}{ Oxygen saturation at peak $\mathrm{VO}_{2}$} \\
\hline 92 & 84 & 88 & & & \\
\hline \multicolumn{6}{|c|}{ Time to peak (min) } \\
\hline 15 & 12 & 10 & & & \\
\hline \multicolumn{6}{|c|}{ Peak WR (Watts) } \\
\hline 99 & 93 & 135 & & & \\
\hline \multicolumn{6}{|c|}{ Peak HR (BPM) } \\
\hline 147 & 132 & 127 & & & \\
\hline \multicolumn{6}{|c|}{$\mathrm{VO} 2(\mathrm{ml} \cdot \mathrm{kg}-1 \cdot \mathrm{min}-1)$} \\
\hline 16 & 14 & 12 & & & \\
\hline \multicolumn{6}{|c|}{ Ve (BPTS) } \\
\hline 65 & 42 & 43 & & & \\
\hline
\end{tabular}


Table E.1: Physiological parameters which were selected to be included in the staging system for every individual

\begin{tabular}{|c|c|c|c|c|c|c|c|c|c|c|c|c|c|}
\hline \multirow[t]{2}{*}{$\begin{array}{c}\text { FVC \% } \\
\text { CATEGO } \\
\text { RY }\end{array}$} & \multirow[t]{2}{*}{$\begin{array}{c}\text { PATIEN } \\
\text { T ID }\end{array}$} & \multirow[t]{2}{*}{ SEX } & \multirow[t]{2}{*}{$\begin{array}{c}\text { FVC } \\
\%\end{array}$} & \multirow[t]{2}{*}{$\begin{array}{c}\text { FEV1 } \\
\%\end{array}$} & \multicolumn{2}{|c|}{ VE (BPTS) (I) } & \multicolumn{2}{|c|}{$\begin{array}{c}\text { VO2 } \\
\left(\mathrm{ml} \cdot \mathrm{kg}^{-1} \cdot \mathrm{min}^{-1}\right)\end{array}$} & \multicolumn{2}{|c|}{$\begin{array}{l}\text { PEAK HR } \\
\text { (BPM) }\end{array}$} & \multicolumn{2}{|c|}{$\begin{array}{c}\text { PEAK WORK } \\
\text { RATE (W) }\end{array}$} & $\begin{array}{r}\text { TIME TO P } \\
\text { WORK RA } \\
\text { (min) }\end{array}$ \\
\hline & & & & & Pre & Post & Pre & Post & Pre & Post & Pre & Post & Pre \\
\hline \multirow[t]{5}{*}{$>80$} & P06 & M & 131 & 130 & 64.7 & 59.7 & 14.6 & 13.5 & 94 & 88 & 81 & 65 & 12.22 \\
\hline & P09 & M & 82 & 85 & 60.5 & 35.2 & 15.1 & 9.6 & 99 & 75 & 93 & 49 & 12.29 \\
\hline & P11 & M & 107 & 93 & 75.3 & 70.5 & 28.4 & 26.2 & 159 & 100 & 148 & 97 & 16.39 \\
\hline & \multicolumn{2}{|l|}{ Mean } & 107 & 103 & 66.8 & 55.1 & 19.37 & 16.4 & 117 & 87.6 & 107 & 70.3 & 13.63 \\
\hline & P07 & $\mathbf{F}$ & 89 & 88 & 64.9 & 43.1 & 16 & 12.5 & 147 & 127 & 99 & 72 & 15.05 \\
\hline \multirow[t]{10}{*}{$55-80$} & P01 & M & 62 & 67 & 74.8 & 79.1 & 14.9 & 15.2 & 124 & 136 & 108 & 109 & 13.11 \\
\hline & P02 & M & 77 & 82 & 30.4 & 38 & 10 & 8.4 & 73 & 75 & 29 & 59 & 11.16 \\
\hline & P03 & M & 63 & 78 & 92.3 & 80.8 & 18.7 & 17.1 & 142 & 125 & 100 & 86 & 12.34 \\
\hline & P08 & M & 74 & 79 & 86.8 & 90.5 & 18.3 & 19.3 & 141 & 111 & 158 & 128 & 13.03 \\
\hline & P14 & $M$ & 59 & 55 & 22.4 & 30.7 & 9.5 & 16.9 & 93 & 46 & 83 & 50 & 7.29 \\
\hline & P17 & $M$ & 78 & 78 & 36 & 63.3 & 14.3 & 17.1 & 115 & 76 & 129 & 99 & 10.43 \\
\hline & \multicolumn{2}{|l|}{ Mean } & 68.8 & 73.2 & 57.1 & 63.7 & 15.5 & 15.4 & 115 & 94.8 & 101 & 88.5 & 11.23 \\
\hline & P04 & $\mathbf{F}$ & 76 & 75 & 49.1 & 47.4 & 13.1 & 12.8 & 138 & 135 & 55 & 62 & 12.07 \\
\hline & P13 & $\mathbf{F}$ & 61 & 63 & 34.7 & 48.2 & 14 & 18.2 & 126 & 65 & 132 & 83 & 12.27 \\
\hline & \multicolumn{2}{|l|}{ Mean } & 68.5 & 69 & 41.9 & 47.8 & 13.6 & 15.5 & 132 & 100 & 93.5 & 72.5 & 12.17 \\
\hline \multirow[t]{2}{*}{$<55$} & P10 & $M$ & 52 & 63 & 52.6 & 53.5 & 14.3 & 12.8 & 101 & 56 & 94 & 35 & 9.2 \\
\hline & P12 & $\mathbf{F}$ & 53 & 60 & 43 & 58.6 & 12.5 & 15.4 & 127 & 72 & 135 & 96 & 10.51 \\
\hline
\end{tabular}




\section{Glossary of Terms}

TERM

Fibrosis

Extracellular Matrix

Interstitium

Minute Ventilation

Dyspnoea

Cardiac Output

Stroke Volume

Sympathetic

Parasympathetic

Diurnal Variation

Metabolic

Biomarker

Palliative Care

Aetiology

Epidemiology

Hypoxaemia

Pulmonary Oedema

Forced Expiratory Volume

Functional Residual Capacity

Arterial Alveolar Gradient

RR Interval

Ventilatory Anaerobic Threshold

Respiratory Exchange Ratio

Aerobic Threshold

\section{DEFINITION}

Thickening and scarring of connective tissue Structure providing support to surrounding cells

Fluid filled space between tissues

Amount of air a person breath in one minute Difficulty in breathing

Amount of blood pumped by the heart in one minute

Volume of blood pumped by the heart in one heart beat

Increasing the heart rate

Reducing the heart rate

Changes occurring during the parts of the day

Self-sustaining chemical reactions in organisms

Naturally occurring molecule or a gene

Care for the terminally ill patient

Cause of a disease condition

Incidence of a disease

Low oxygen saturation in blood

Excess fluid in lungs

Volume of air a person can exhale during a forced breath

Volume of air present in the lung after passive expiration

The difference between alveolar concentration of oxygen and arterial concentration of oxygen Interval between successive heart beats Point during exercise at which ventilation starts to increase at a faster rate than inspiration Ratio between the amount of carbon dioxide produced and oxygen used Intensity at which lactate accumulates during exercise 


\section{References}

1. Murray LA. Commonalities between the pro- fibrotic mechanisms in COPD and IPF. PulmPharmacol \& Ther. 2012;25(4):276.

2. Raghu G, Collard HR, Egan JJ, Martinez FJ, Behr J, Brown KK, et al. An official ATS/ERS/JRS/ALAT statement: idiopathic pulmonary fibrosis: evidence- based guidelines for diagnosis and management. Am J Respir Crit Care Med. 2011;183(6):788.

3. Travis WD, Costabel U, Hansell DM, King TE, Lynch DA, Nicholson AG, et al. An official American Thoracic Society/ European Respiratory Society statement: Update of the international multidisciplinary classification of the idiopathic interstitial pneumonias. Am J Respir Crit Care Med. 2013;188(6):733.

4. Ley B, Collard HR, King TE, Jr. Clinical course and prediction of survival in idiopathic pulmonary fibrosis. Am J Respir Crit Care Med. 2011;183(4):431-40.

5. Lederer DJ, Martinez FJ. Idiopathic Pulmonary Fibrosis. N Engl J Med. 2018;378(19):1811-23.

6. Decramer M, Janssens W, Miravitlles M. Chronic obstructive pulmonary disease. The Lancet. 2012;379(9823):1341-51.

7. Burns N. COPD and pulmonary rehabilitation. Aust Fam Physician. 2012;41(7):460.

8. Spruit MA. An official American Thoracic Society/ European Respiratory Society statement: key concepts and advances in pulmonary rehabilitation. Am J Respir Crit Care Med. 2013;188(8):e13.

9. American Thoracic Society. Idiopathic pulmonary fibrosis: diagnosis and treatment. International consensus statement. American Thoracic Society (ATS), and the European Respiratory Society (ERS). Am J Respir Crit Care Med. 2000;161(2 Pt 1):646-64.

10. Bolton CE. British Thoracic Society guideline on pulmonary rehabilitation in adults: accredited by NICE. Thorax. 2013;68(9):ii1.

11. Dowman LM, McDonald CF, Hill CJ, Lee AL, Barker K, Boote C, Glaspole I, Goh NS, Southcott AM, Burge AT, Gillies R. The evidence of benefits of exercise training in interstitial lung disease: a randomised controlled trial. Thorax. 2017;72(7):610-9.

12. Tonelli R, Cocconcelli E, Lanini B, Romagnoli I, Florini F, Castaniere I, et al. Effectiveness of pulmonary rehabilitation in patients with interstitial lung disease of different etiology: a multicenter prospective study. BMC Pulm Med2017. 
13. Dowman L. Pulmonary rehabilitation for interstitial lung disease. Cochrane Database Syst Rev. 2014(10):CD006322.

14. Vainshelboim B, Oliveira J, Fox BD, Soreck Y, Fruchter O, Kramer MR. Long-term effects of a 12 -week exercise training program on clinical outcomes in idiopathic pulmonary fibrosis. Lung. 2015;193(3):345-54.

15. Holland AE, Hill CJ, Conron M, Munro P, McDonald CF. Short term improvement in exercise capacity and symptoms following exercise training in interstitial lung disease. Thorax. 2008;63(6):549-54.

16. McNarry MA, Harrison NK, Withers T, Chinnappa N, Lewis MJ. Pulmonary oxygen uptake and muscle deoxygenation kinetics during heavy intensity cycling exercise in patients with emphysema and idiopathic pulmonary fibrosis. BMC Pulm Med 2017;17(1):26.

17. Baba R, Nagashima M, Goto M, Nagano Y, Yokota M, Tauchi N, et al. Oxygen uptake efficiency slope: a new index of cardiorespiratory functional reserve derived from the relation between oxygen uptake and minute ventilation during incremental exercise. J Am Coll Cardiol. 1996;28(6):1567-72.

18. Baba R. The oxygen uptake efficiency slope and its value in the assessment of cardiorespiratory functional reserve. Congest Heart Fail. 2000;6(5):256-8.

19. Akkerman M, van Brussel M, Hulzebos E, Vanhees L, Helders PJ, Takken T. The oxygen uptake efficiency slope: what do we know? J Cardiopulm Rehabil Prev. 2010;30(6):357-73.

20. Hollenberg M, Tager IB. Oxygen uptake efficiency slope: an index of exercise performance and cardiopulmonary reserve requiring only submaximal exercise. J Am Coll Cardiol. 2000;36(1):194-201.

21. Davies LC, Wensel R, Georgiadou P, Cicoira M, Coats AJ, Piepoli MF, et al. Enhanced prognostic value from cardiopulmonary exercise testing in chronic heart failure by non-linear analysis: oxygen uptake efficiency slope. Eur Heart J. 2006;27(6):684-90.

22. Marinov B, Mandadzhieva S, Kostianev S. Oxygen-uptake efficiency slope in healthy 7-to 18year-old children. Pediatr Exerc Sci. 2007 19(2):159-70.

23. Whipp BJ, Ward SA. Physiological determinants of pulmonary gas exchange kinetics during exercise. Med Sci Sports Exerc. 1990;22(1):62-71.

24. Puente-Maestu L, Sanz ML, Sanz P, Ruiz de Ona JM, Rodriguez-Hermosa JL, Whipp BJ. Effects of two types of training on pulmonary and cardiac responses to moderate exercise in patients with COPD. Eur Respir J. 2000;15(6):1026-32. 
25. Vainshelboim B. Exercise training- based pulmonary rehabilitation program is clinically beneficial for idiopathic pulmonary fibrosis. Respiration. 2014;88(5):378.

26. MacPhee SL, Shoemaker JK, Paterson DH, Kowalchuk JM. Kinetics of O2 uptake, leg blood flow, and muscle deoxygenation are slowed in the upper compared with lower region of the moderate-intensity exercise domain. J Appl Physiol (1985). 2005;99(5):1822-34.

27. Yoshida T, Whipp BJ. Dynamic asymmetries of cardiac output transients in response to muscular exercise in man. J Physiol. 1994;480(Pt 2):355-9.

28. Lewis MJ, Short AL. Exercise and cardiac regulation: what can electrocardiographic time series tell us? Scand J Med Sci Sports. 20(6):794-804.

29. Bauer A, Kantelhardt JW, Bunde A, Barthel P, Schneider R, Malik M, et al. Phase-rectified signal averaging detects quasi-periodicities in non-stationary data. Physica A. 2006;364:423-34.

30. Bartels MN, Jelic S, Ngai P, Basner RC, DeMeersman RE. High-frequency modulation of heart rate variability during exercise in patients with COPD. Chest. 2003;124(3):863-9.

31. Lewis MJ, Annandale J, Lewis KE. Influence of long-term oxygen therapy on heart rate and QT time-series in hypoxic patients with chronic obstructive pulmonary disease. Clin Physiol Funct Imaging. 2009;29(6):431-9.

32. Richeldi L. Diagnosis and treatment of idiopathic pulmonary fibrosis part 1 of 2 / Luca Richeldi. London: London : Henry Stewart Talks; 2015.

33. Snell N, Strachan D, Hubbard R, Gibson J, Maher T, Jarrold I. P272 Epidemiology of idiopathic pulmonary fibrosis in the uk: findings from the british lung foundation's 'respiratory health of the nation' project. 2016.

34. Gribbin J. Incidence and mortality of idiopathic pulmonary fibrosis and sarcoidosis in the UK. Thorax. 2006;61(11):980.

35. Navaratnam V. The increasing secondary care burden of idiopathic pulmonary fibrosis: hospital admission trends in England from 1998 to 2010. Chest. 2013;143(4):1078.

36. Visscher DW. Histologic spectrum of idiopathic interstitial pneumonias. Proc Am Thorac Soc. 2006;3(4):322.

37. Churg A. Acute exacerbation ( acute lung injury of unknown cause) in UIP and other forms of fibrotic interstitial pneumonias. Am J Surg Pathol. 2007;31(2):277.

38. King TE, Pardo A, Selman M. Idiopathic pulmonary fibrosis. The Lancet. 2011;378(9807):194961. 
39. Longo DL, Rockey DC, Bell PD, Hill JA. Fibrosis - A Common Pathway to Organ Injury and Failure. N Engl J Med. 2015;372(12):1138-49.

40. Baumgartner KB, Samet JM, Coultas DB, Stidley CA, Hunt WC, Colby TV, et al. Occupational and environmental risk factors for idiopathic pulmonary fibrosis: a multicenter case-control study. Collaborating Centers. Am J Epidemiol. 2000;152(4):307-15.

41. Demedts M, Wells AU, Anto JM, Costabel U, Hubbard R, Cullinan P, et al. Interstitial lung diseases: an epidemiological overview. Eur Respir J Suppl. 2001;32:2s-16s.

42. Tobin RW, Pope CE, 2nd, Pellegrini CA, Emond MJ, Sillery J, Raghu G. Increased prevalence of gastroesophageal reflux in patients with idiopathic pulmonary fibrosis. Am J Respir Crit Care Med. 1998;158(6):1804-8.

43. Patti MG, Tedesco P, Golden J, Hays S, Hoopes C, Meneghetti A, et al. Idiopathic pulmonary fibrosis: how often is it really idiopathic? J Gastrointest Surg. 2005;9(8):1053-6; discussion 68.

44. Moore BB, Moore TA. Viruses in Idiopathic Pulmonary Fibrosis. Etiology and Exacerbation. Ann Am Thorac Soc. 2015;12 Suppl 2:S186-92.

45. Dempsey OJ. Clinical review: idiopathic pulmonary fibrosis - past, present and future. Respir Med. 2006 Nov 1;100(11):1871-85.

46. Raghu G, Weycker D, Edelsberg J, Bradford WZ, Oster G. Incidence and prevalence of idiopathic pulmonary fibrosis. Am J Respir Crit Care Med. 2006;174(7):810.

47. Coultas DB, Zumwalt RE, Black WC, Sobonya RE. The epidemiology of interstitial lung diseases. Am J Respir Crit Care Med. 1994;150(4):967-72.

48. Natsuizaka M, Chiba H, Kuronuma K, Otsuka M, Kudo K, Mori M, et al. Epidemiologic survey of Japanese patients with idiopathic pulmonary fibrosis and investigation of ethnic differences. Am J Respir Crit Care Med. 2014;190(7):773-9.

49. Flaherty KR, Travis WD, Colby TV, Toews GB, Kazerooni EA, Gross BH, et al. Histopathologic variability in usual and nonspecific interstitial pneumonias. Am J Respir Crit Care Med. 2001;164(9):1722-7.

50. Collard HR, Moore BB, Flaherty KR, Brown KK, Kaner RJ, King TE, Jr., et al. Acute exacerbations of idiopathic pulmonary fibrosis. Am J Respir Crit Care Med. 2007;176(7):636-43.

51. Tomioka H, Sakurai T, Hashimoto K, Iwasaki H. Acute exacerbation of idiopathic pulmonary fibrosis: role of Chlamydophila pneumoniae infection. Respirology. 2007;12(5):700-6. 
52. King TE, Jr., Albera C, Bradford WZ, Costabel U, Hormel P, Lancaster L, et al. Effect of interferon gamma-1b on survival in patients with idiopathic pulmonary fibrosis (INSPIRE): a multicentre, randomised, placebo-controlled trial. Lancet. 2009;374(9685):222-8.

53. Song JW, Hong SB, Lim CM, Koh Y, Kim DS. Acute exacerbation of idiopathic pulmonary fibrosis: incidence, risk factors and outcome. Eur Respir J. 2011;37(2):356-63.

54. Hamman L, Rich AR. Fulminating Diffuse Interstitial Fibrosis of the Lungs. Trans Am Clin Climatol Assoc. 1935;51:154-63.

55. Noble PW, Homer RJ. Back to the future: historical perspective on the pathogenesis of idiopathic pulmonary fibrosis. Am J Respir Cell Mol Biol. 2005;33(2):113-20.

56. Selman M, King TE, Pardo A. Idiopathic pulmonary fibrosis: prevailing and evolving hypotheses about its pathogenesis and implications for therapy. Ann Intern Med. 2001;134(2):136-51.

57. Davies HR, Richeldi L, Walters EH. Immunomodulatory agents for idiopathic pulmonary fibrosis. Cochrane Database Syst Rev. 2003(3):Cd003134.

58. Richeldi L, Davies HR, Ferrara G, Franco F. Corticosteroids for idiopathic pulmonary fibrosis. Cochrane Database Syst Rev. 2003(3):Cd002880.

59. Selman M, Thannickal VJ, Pardo A, Zisman DA, Martinez FJ, Lynch JP, 3rd. Idiopathic pulmonary fibrosis: pathogenesis and therapeutic approaches. Drugs. 2004;64(4):405-30.

60. Selman $M$, Pardo A. Role of epithelial cells in idiopathic pulmonary fibrosis: from innocent targets to serial killers. Proc Am Thorac Soc. 2006;3(4):364-72.

61. Egan JJ, Martinez FJ, Wells AU, Williams T. Lung function estimates in idiopathic pulmonary fibrosis: the potential for a simple classification. Thorax. England2005. p. 270-3.

62. Schwartz DA, Helmers RA, Galvin JR, Van Fossen DS, Frees KL, Dayton CS, et al. Determinants of survival in idiopathic pulmonary fibrosis. Am J Respir Crit Care Med. 1994;149(2 Pt 1):4504.

63. Erbes R, Schaberg T, Loddenkemper R. Lung function tests in patients with idiopathic pulmonary fibrosis. Are they helpful for predicting outcome? Chest. 1997;111(1):51-7.

64. Schwartz DA, Van Fossen DS, Davis CS, Helmers RA, Dayton CS, Burmeister LF, et al. Determinants of progression in idiopathic pulmonary fibrosis. Am J Respir Crit Care Med. 1994;149(2 Pt 1):444-9. 
65. Hanley ME, King TE, Jr., Schwarz MI, Watters LC, Shen AS, Cherniack RM. The impact of smoking on mechanical properties of the lungs in idiopathic pulmonary fibrosis and sarcoidosis. Am Rev Respir Dis. 1991;144(5):1102-6.

66. Ostrow D, Cherniack RM. Resistance to airflow in patients with diffuse interstitial lung disease. Am Rev Respir Dis. 1973;108(2):205-10.

67. Kornbluth RS, Turino GM. Respiratory control in diffuse interstitial lung disease and diseases of the pulmonary vasculature. Clin Chest Med. 1980;1(1):91-102.

68. Renzi G, Milic-Emili J, Grassino AE. The pattern of breathing in diffuse lung fibrosis. Bull Eur Physiopathol Respir. 1982;18(3):461-72.

69. Lourenco RV, Turino GM, Davidson LA, Fishman AP. The regulation of ventilation in diffuse pulmonary fibrosis. Am J Med. 1965;38:199-216.

70. Patton JM, Freedman S. The ventilatory response to $\mathrm{CO} 2$ of patients with diffuse pulmonary infiltrations or fibrosis. Clin Sci. 1972 1;43(1):55-69.

71. Pardo A, Selman M. Idiopathic pulmonary fibrosis: new insights in its pathogenesis. Int J Biochem Cell Biol. 2002;34(12):1534-8.

72. Rudd RM, Haslam PL, Turner-Warwick M. Cryptogenic fibrosing alveolitis. Relationships of pulmonary physiology and bronchoalveolar lavage to response to treatment and prognosis. Am Rev Respir Dis. 1981;124(1):1-8.

73. Meier-Sydow J, Rust M, Kronenberger H, Thiel C, Amthor M, Riemann H. Long-term follow-up of lung function parameters in patients with idiopathic pulmonary fibrosis treated with prednisone and azathioprin or d-penicillamine (author's transl). Prax Klin Pneumol. 1979 33:680-8.

74. Winterbauer RH, Hammar SP, Hallman KO, Hays JE, Pardee NE, Morgan EH, et al. Diffuse interstitial pneumonitis. Clinicopathologic correlations in 20 patients treated with prednisone/azathioprine. Am J Med. 1978;65(4):661-72.

75. Berry MJ. A randomized, controlled trial comparing long- term and short- term exercise in patients with chronic obstructive pulmonary disease. J Cardiopulm Rehabil. 2003;23(1):60.

76. Peters SG, McDougall JC, Douglas WW, Coles DT, DeRemee RA. Colchicine in the treatment of pulmonary fibrosis. Chest. 1993;103(1):101-4.

77. Rennard SI, Bitterman PB, Ozaki T, Rom WN, Crystal RG. Colchicine suppresses the release of fibroblast growth factors from alveolar macrophages in vitro. The basis of a possible therapeutic approach ot the fibrotic disorders. Am Rev Respir Dis. 1988;137(1):181-5. 
78. Sewell L, Singh SJ, Williams JEA, Collier R, Morgan MDL. Can individualized rehabilitation improve functional independence in elderly patients with COPD? Rev Port Pneumol. 2005;11(6):593.

79. Green RH, Singh SJ, Williams J, Morgan MDL. A randomised controlled trial of four weeks versus seven weeks of pulmonary rehabilitation in chronic obstructive pulmonary disease. Thorax. 2001;56(2):143.

80. Solanes I. Duration of pulmonary rehabilitation to achieve a plateau in quality of life and walk test in COPD. Respir Med. 2009;103(5):722.

81. Swallow EB. Quadriceps strength predicts mortality in patients with moderate to severe chronic obstructive pulmonary disease. Thorax. 2007;62(2):115.

82. Bernard S. Aerobic and strength training in patients with chronic obstructive pulmonary disease. Am J Respir Crit Care Med. 1999;159(3):896.

83. Mador MJ. Endurance and strength training in patients with COPD. Chest. 2004;125(6):2036.

84. Vonbank K. Strength training increases maximum working capacity in patients with COPD-randomized clinical trial comparing three training modalities. Respir Med. 2012;106(4):557.

85. Fischer MJ. Drop- out and attendance in pulmonary rehabilitation: the role of clinical and psychosocial variables. Respir Med. 2009;103(10):1564.

86. Bjoernshave B. Does pulmonary rehabilitation work in clinical practice? A review on selection and dropout in randomized controlled trials on pulmonary rehabilitation. Clin Epidemiol. 2010;2:73.

87. Duck A, Spencer LG, Bailey S, Leonard C, Ormes J, Caress AL. Perceptions, experiences and needs of patients with idiopathic pulmonary fibrosis. J Adv Nurs. 2015;71(5):1055-65.

88. Bajwah S, Higginson IJ, Ross JR, Wells AU, Birring SS, Riley J, et al. The palliative care needs for fibrotic interstitial lung disease: a qualitative study of patients, informal caregivers and health professionals. Palliat Med. 2013;27(9):869-76.

90. Schoenheit G, Becattelli I, Cohen AH. Living with idiopathic pulmonary fibrosis: an in-depth qualitative survey of European patients. Chron Respir Dis. 2011;8(4):225-31.

91. Mahler DA. Pulmonary rehabilitation. Chest. 1998;113(4 Suppl):263s-8s. 
92. Kozu R, Senjyu H, Jenkins SC, Mukae H, Sakamoto N, Kohno S. Differences in response to pulmonary rehabilitation in idiopathic pulmonary fibrosis and chronic obstructive pulmonary disease. Respiration. 2011;81(3):196-205.

93. Kozu R, Jenkins S, Senjyu H. Effect of disability level on response to pulmonary rehabilitation in patients with idiopathic pulmonary fibrosis. Respirology. 2011;16(8):1196-202.

94. Nishiyama O, Kondoh $\mathrm{Y}$, Kimura $\mathrm{T}$, Kato K, Kataoka K, Ogawa $\mathrm{T}$, et al. Effects of pulmonary rehabilitation in patients with idiopathic pulmonary fibrosis. Respirology. 2008;13(3):394-9.

95. Swigris JJ, Fairclough DL, Morrison M, Make B, Kozora E, Brown KK, Wamboldt FS. Benefits of pulmonary rehabilitation in idiopathic pulmonary fibrosis. Respir Care. 2011 56(6):783-9.

96. Holland AE, Hill CJ, Glaspole I, Goh N, McDonald CF. Predictors of benefit following pulmonary rehabilitation for interstitial lung disease. Respir Med. 2012;106(3):429-35.

97. Ozalevli S, Karaali HK, Ilgin D, Ucan ES. Effect of home-based pulmonary rehabilitation in patients with idiopathic pulmonary fibrosis. Multidiscip Respir Med2010. p. 31-7.

98. Kenn K. Pulmonary rehabilitation in patients with idiopathic pulmonary fibrosis--a review. Respiration; Respiration. 2013;86(2):89.

99. Lewis MJ, Kingsley M, Short AL, Simpson K. Rate of reduction of heart rate variability during exercise as an index of physical work capacity. Scand J Med Sci Sports. 2007;17(6):696-702.

100. Lewis MJ. Heart Rate Variability Analysis: A Tool to Assess Cardiac Autonomic Function. Comput Inform Nurs. 2019;23(6):335-41.

101. Heart rate variability: standards of measurement, physiological interpretation and clinical use. Task Force of the European Society of Cardiology and the North American Society of Pacing and Electrophysiology. Circulation. 1996;93(5):1043-65.

102. Campana LM, Owens RL, Clifford GD, Pittman SD, Malhotra A. Phase-rectified signal averaging as a sensitive index of autonomic changes with aging. J Appl Physiol (1985). 2010;108(6):166873.

103. Bauer A, Kantelhardt JW, Barthel P, Schneider R, Makikallio T, Ulm K, et al. Deceleration capacity of heart rate as a predictor of mortality after myocardial infarction: cohort study. Lancet. 2006;367(9523):1674-81.

104. Kantelhardt JW, Bauer A, Schumann AY, Barthel P, Schneider R, Malik M, et al. Phase-rectified signal averaging for the detection of quasi-periodicities and the prediction of cardiovascular risk. Chaos. 2007;17(1):015112. 
105. Lewis MJ, Annandale J, D'Silva LA, Davies RE, Reed Z, Lewis KE. Influence of long-term oxygen therapy on cardiac acceleration and deceleration capacity in hypoxic patients with chronic obstructive pulmonary disease. Clin Physiol Funct Imaging. 2011;31(4):258-65.

106. Puente-Maestu L, Sanz ML, Sanz P, De Ona JR, Rodriguez-Hermosa JL, Whipp BJ. Effects of two types of training on pulmonary and cardiac responses to moderate exercise in patients with COPD. Eur Respir J. 2000 15(6):1026-32.

107. McNarry MA, Harrison NK, Withers T, Chinnappa N, Lewis MJ. Pulmonary oxygen uptake and muscle deoxygenation kinetics during heavy intensity cycling exercise in patients with emphysema and idiopathic pulmonary fibrosis. BMC Pulmonary Medicine. 2017;17(1):26.

108. Jackson RM, Gomez-Marin OW, Ramos CF, Sol CM, Cohen MI, Gaunaurd IA, et al. Exercise limitation in IPF patients: a randomized trial of pulmonary rehabilitation. Lung. 2014;192(3):367-76.

109. Gross TJ, Hunninghake GW. Idiopathic pulmonary fibrosis. N Engl J Med. 2001;345(7):517-25.

110. Thomas DR. A general inductive approach for analyzing qualitative evaluation data. Am J Eval. 2006 Jun;27(2):237-46.

111. Ryan RM, Deci EL. Self-determination theory and the facilitation of intrinsic motivation, social development, and well-being. Am Psychol. 2000;55(1):68.

112. Ntoumanis N, Edmunds J, Duda JL. Understanding the coping process from a selfdetermination theory perspective. Br J Health Psychol. 200914(2):249-60.

113. Lazarus RS. Emotion and Adaptation; Oxford University Press, 1994.

114. selfdeterminationtheory.org - An Approach to human motivation \& personality [Internet]. 2017. Available from: http://selfdeterminationtheory.org/.

115. Rahman RJ, Thogersen-Ntoumani C, Thatcher J, Doust J. Changes in need satisfaction and motivation orientation as predictors of psychological and behavioural outcomes in exercise referral. Psychol Health. 2011;26(11):1521-39.

116. Rahman RJ, Hudson J, Thogersen-Ntoumani C, Doust JH. Motivational processes and wellbeing in cardiac rehabilitation: a self-determination theory perspective. Psychol Health Med. 2015;20(5):518-29.

117. Ntoumanis N, Edmunds J, Duda JL. Understanding the coping process from a selfdetermination theory perspective. Br J Health Psychol 2009. 14(Pt 2):249-260. 
118. Stein NL, Leventhal B, Trabasso TR, editors. Psychological and biological approaches to emotion. Lawrence Erlbaum Asociates, Publishers; 1990 .

119. Holahan CJ, Moos RH, Holahan CK, Brennan PL, Schutte KK. Stress Generation, Avoidance Coping, and Depressive Symptoms: A 10-Year Model. J Consult Clin Psychol. 2005;73(4):65866.

120. Aspinwall LG, Taylor SE. A stitch in time: Self-regulation and proactive coping. Psychol Bull. 1997121(3):417..

121. Matthieu MM, Ivanoff A. Using stress, appraisal, and coping theories in clinical practice: Assessments of coping strategies after disasters. Brief Treat Crisis Interv. 2006 6(4):337.

122. Laugaa D, Rascle N, Bruchon-Schweitzer M. Stress and burnout among French elementary school teachers: A transactional approach. Eur Rev Appl Psychol. 2008 1;58(4):241-51.

123. Rubin HJ, Rubin IS. Qualitative interviewing: The art of hearing data. Sage publications; 2011 .

124. Braun V, Clarke V. Using thematic analysis in psychology. Qual Res Psychol. 2006 3(2):77-101.

125. Miles MB, Huberman AM, Huberman MA, Huberman M. Qualitative data analysis: An expanded sourcebook. sage; 1994 Jan 12.

126. Herdman M. Development and preliminary testing of the new five- level version of EQ- 5D ( EQ- 5D- 5L). Quality of Life Research: Qual Life Res. 2011;20(10):1727-37.

127. Zigmond AS, Snaith RP. The hospital anxiety and depression scale. Acta Psychiatr Scand. 1983;67(6):361-70.

128. Bestall JC. Usefulness of the Medical Research Council (MRC) dyspnoea scale as a measure of disability in patients with chronic obstructive pulmonary disease. Thorax. 1999;54(7):581.

129. Patel AS. The development and validation of the King's Brief Interstitial Lung Disease ( K-BILD) health status questionnaire. Thorax. 2012;67(9):804.

130. Torrey B. ATS guidelines for the six-minute walk test.(American Thoracic Society Committee on Proficiency Standards for Clinical Pulmonary Function Laboratories). Am Fam Physician. 2002;66(5):904.

131. Erratum: ATS Statement: Guidelines for the Six-Minute Walk Test. Am J Respir Crit Care Med. 2016;193(10):1185.

132. Arena R. Cardiopulmonary exercise testing in the clinical evaluation of patients with heart and lung disease. Circulation. 2011;123(6):668. 
133. Castro RR, Pedrosa S, Chabalgoity F, Sousa EB, Nobrega AC. The influence of a fast ramp rate on peak cardiopulmonary parameters during arm crank ergometry. Clin Physiol Funct Imaging. 2010;30(6):420-5.

134. Tarvainen MP, Niskanen J-P, Lipponen JA, Ranta-aho PO, Karjalainen PA. Kubios HRV - Heart rate variability analysis software. Comput Methods Programs Biomed. 2014;113(1):210-20.

135. Electrophysiology TF. Heart rate variability: standards of measurement, physiological interpretation, and clinical use. Circulation. 1996 93(5):1043-65.

136. Cummin AR, lyawe VI, Mehta N, Saunders KB. Ventilation and cardiac output during the onset of exercise, and during voluntary hyperventilation, in humans. J Physiol. 1986;370:567-83.

137. Bell C, Paterson DH, Kowalchuk JM, Padilla J, Cunningham DA. A comparison of modelling techniques used to characterise oxygen uptake kinetics during the on-transient of exercise. Exp Physiol. 2001;86(5):667-76.

138. Russell A-M, Ripamonti E, Vancheri C. Qualitative European survey of patients with idiopathic pulmonary fibrosis: patients' perspectives of the disease and treatment. BMC Pulm Med. 2016;16(1):10.

139. Sampson C, Gill BH, Harrison NK, Nelson A, Byrne A. The care needs of patients with idiopathic pulmonary fibrosis and their carers (CaNoPy): results of a qualitative study. BMC Pulm Med2015.

140. Swigris JJ, Stewart AL, Gould MK, Wilson SR. Patients' perspectives on how idiopathic pulmonary fibrosis affects the quality of their lives. Health Qual Life Outcomes. 2005;3(1):61.

141. Bonella F, Wijsenbeek M, Molina-Molina M, Duck A, Mele R, Geissler K. European IPF Patient Charter: unmet needs and a call to action for healthcare policymakers. Eur Respir J. 2016;47(2):597-606.

142. Cicutto L, Brooks D, Henderson K. Self-care issues from the perspective of individuals with chronic obstructive pulmonary disease. Patient Educ Couns. 2004 55(2):168-76.

143. Fernandez S, Franklin J, Amlani N, DeMilleVille C, Lawson D, Smith J. Physical activity and cancer: A cross-sectional study on the barriers and facilitators to exercise during cancer treatment. Can Oncol Nurs J. 201525(1):37-42.

144. Overgaard D, Kaldan G, Marsaa K, Nielsen TL, Shaker SB, Egerod I. The lived experience with idiopathic pulmonary fibrosis: a qualitative study. Eur Respir J. 2016;47(5):1472-80.

145. Ramponi S, Tzani P, Aiello M, Marangio E, Clini E, Chetta A. Pulmonary Rehabilitation Improves Cardiovascular Response to Exercise in COPD. Respiration. 2018;86(1):17-24. 
146. Cottin V, Richeldi L. Neglected evidence in idiopathic pulmonary fibrosis and the importance of early diagnosis and treatment. Eur Respir Rev. 2014;23(131):106-10.

147. Tulppo MP, Makikallio TH, Takala TE, Seppanen T, Huikuri HV. Quantitative beat-to-beat analysis of heart rate dynamics during exercise. Am J Physiol. 1996;271(1 Pt 2):H244-52.

148. Perini R, Milesi S, Fisher NM, Pendergast DR, Veicsteinas A. Heart rate variability during dynamic exercise in elderly males and females. Eur J Appl Physiol. 2000;82(1-2):8-15.

149. McNarry MA, Lewis MJ. Interaction between age and aerobic fitness in determining heart rate dynamics. Physiol Meas. 2012 33(6):901.

150. Breese BC, Barker AR, Armstrong N, Jones AM, Williams CA. The effect of baseline metabolic rate on pulmonary $\mathrm{O}(2)$ uptake kinetics during very heavy intensity exercise in boys and men. Respir Physiol Neurobiol. 2012;180(2-3):223-9.

151. Rowland TW, Cunningham LN. Oxygen uptake plateau during maximal treadmill exercise in children. Chest. 1992;101(2):485-9.

152. Weber KT, Kinasewitz GT, Janicki JS, Fishman AP. Oxygen utilization and ventilation during exercise in patients with chronic cardiac failure. Circulation. 1982;65(6):1213-23.

153. Kolb M, Collard HR. Staging of idiopathic pulmonary fibrosis: past, present and future. Eur Respir Rev. 2014;23(132):220-4.

154. Gonnella JS, Hornbrook MC, Louis DZ. Staging of disease. A case-mix measurement. Jama. 1984;251(5):637-44.

155. Ekberg-Aronsson M, Pehrsson K, Nilsson J, Nilsson PM, Löfdahl CG. Mortality in GOLD stages of COPD and its dependence on symptoms of chronic bronchitis. Respir Res2005. p. 98.

156. Vestbo J, Hurd SS, Agusti AG, Jones PW, Vogelmeier C, Anzueto A, et al. Global strategy for the diagnosis, management, and prevention of chronic obstructive pulmonary disease: GOLD executive summary. Am J Respir Crit Care Med. 2013;187(4):347-65.

157. du Bois RM, Weycker D, Albera C, Bradford WZ, Costabel U, Kartashov A, et al. Ascertainment of individual risk of mortality for patients with idiopathic pulmonary fibrosis. Am J Respir Crit Care Med. 2011;184(4):459-66.

158. Ley B, Ryerson CJ, Vittinghoff E, Ryu JH, Tomassetti S, Lee JS, et al. A multidimensional index and staging system for idiopathic pulmonary fibrosis. Ann Intern Med. 2012;156(10):684-91. 
